Forschungsberichte des Instituts fü deutsche Sprache Ulrich Engel - Helmut Schumacher

\title{
Kleines Valenzlexikon deutscher Verben
}

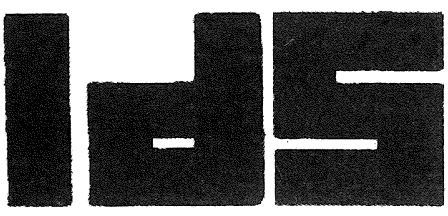

EN Gunter Narr Vorlag Tübingen 
Der Verlag bedauert, $\mathrm{da} \beta$ bei der technischen Fertigstellung der 2. Auflage einige Fehler unterlaufen sind. Wir bitten, folgende Berichtigungen vorzunehmen:

S. 41, linke Spalte:

Ergänze den 1. Absatz: Alleiniges Kriterium für Transitivität ist dabei das Vorliegen eines Akkusativs.

S. 70, linke spalte:

Die Zeile vor 5.6.4. ist zu streichen.

S. 82 , rechte spalte:

Zeile 2-5 lies: Dort ist beispielsweise vermerkt "1:SE..."; der darauffolgende Beispielsatz enthält also eine $E_{1}$ in Form einer $S E$. Im KVL erscheinen die folgenden SE-Klassen:

S. 129, Verbspalte zweite Zeile von unten:

Ergänze aufmachen.

S. 164, eilen SBP 06:

Die Rundklammer und der zweite Punkt nach dem Beispielsatz sind zu streichen.

S. 173:

Die letzte zeile vor erwarten ist zu streichen.

S. 193, vierte Zeile von unten:

Ergänze hinter halten SBP 01 und PO.

S. 206, Verbspalte Zeile 7:

kennenlermen ist zu streichen.

S. 284, Verbspalte Zeile 2:

Die Klammern sind zu streichen. 
Ulrich Engel/Helmut Schumacher

\section{Kleines Valenzlexikon}




\section{FORSCHUNGSBERICHTE DES}

INSTITUTS FUR DEUTSCHE SPRACHE

\section{MANNHEIM}

herausgegeben von

Ulrich Engel und Gerhard Stickel

Schriftleitung: Eva Teubert

Band 31 


\section{Ulrich Engel - Helmut Schumacher}

\section{Kleines Valenzlexikon deutscher Verben}

unter Mitarbeit von

Joachim Ballweg Angelika Ballweg-Schramm - Bernd Ulrich Biere

Heide Günther · Hans-Jürgen Hacker - Günther A. Hamel - Anne Heußner - Brigitte Hilgendorf

Inken Keim · Karlheinz Köhler · Sabine Pape - Norbert Trautz u. a.

(TB.) TBL Verlag Gunter Narr - Tübingen

Tübingen 1978 


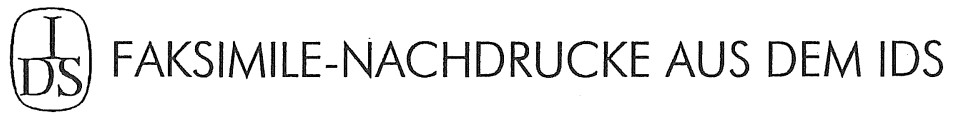

ISBN: 3-922641-84-9

(C) 2003 Institut für Deutsche Sprache, R 5, 6-13, D-68161 Mannheim

http://www.ids-mannheim.de

Mitglied der $1 / 2$ Leibniz

Genehmigter Nachdruck der unter folgendem Impressum erschienenen Ausgabe:

2. durchgesehene Auflage 1978

ISBN 3-87808-631-8

(C) 1976 TBD Verlag Gunter Narr · Tübingen

Alle Rechte vorbehalten. Nachdruck oder Vervielfältigung, auch auszugsweise, in allen Formen wie Mikrofilm, Xerographie, Mikrofiche, Mikrocord, Offset verboten.

Druck: Müller+Bass $\cdot 74$ Tübingen $\cdot$ Hechinger Straße 25

Das Werk einschließlich seiner Teile ist urheberrechtlich geschützt. Jede Verwertung außerhalb der engen Grenzen des Urheberrechts ist ohne Zustimmung der Copyright-Inhaber unzulässig und strafbar. Das gilt insbesondere für Vervielfältigungen, Übersetzungen, Mikroverfilmungen und die Einspeicherung und Verarbeitung in elektronischen Systemen.

Herstellung und Vertrieb im Eigenverlag.

Printed in Germany 
INHALT

Vorwort

Einleitung

Literaturverzeichnis

Register

Abkürzungsverzeichnis

Wörterbuch 



\section{VORWORT}

Dieses Valenzlexikon tst in erster Linie als ein Hilfsmittel fur Lehrer und Lehrbuchautoren gedacht, die sich mit der Vermittlung des Deutschen vor allem auf der Grundstufe beschäftigen. Diesen potentiellen Henutzern soll es die Möglichkeit eröffnen, das Lehrmaterial für einen wichtigen Bereich der Grammatikubungen in umfassender Wefse zusammenzustellen.

Wegen dex sparsamen Frläutexungen setzt das richtige Verstehen der Wörterbucheinträge in der Regel einen Grad von Kompetenz im Deutschen voraus, uber den ein schüler nicht unbedingt verfigt. Trotzdem soll nicht ausgeschlossen werden, daß auch ein fortgeschrittener Schüler dieses Buch mit Gewinn zu Rate ztehen kann. Man muß jedoch nachdricklich davor warnen, dieses Buch als Mittel zum Selbststudiun oder als Ersatz für ein Lehrbuch zu betrachten. Es ist vielmehr ein Nachschlagewerk und sollte als solches benutzt werden.

Dle Auswahl und die Anordnung der im wörterbuch gegebenen Informationen sowtie die Form threx Darstellung sind theoriegebunden. Daher kann nicht erwartet werden; daB thre Informationen bereits in elner didaktisch angemessenen Form vorgefunden werden. Die nuswahl und didaktische vermittlung muB dalier in jedem Fall vom produzenten des Lehrmaterials geleistet werden.

Es ist unvermeidlich, daB der kompetente Benutzer dieses wörterbuchs in manchen Fällen $z u$ efner anderen Beurtellung der strukturen kommt, als sie hier angegeben sind. Wir haben bei der Erstellung des wörterbuchs tmmer wieder die Feststellung treffen können, daB die Beurteflung von Sätzen auf Grund von Kompetenzentscheidungen bei einer größeren Gruppe von kompetenten Sprechern zu manchmal sehr unterschied11chen Ergebnissen führen kann. Wir haben versucht, nur solche strukturen aufzuneh- 
men, deren Grammatikalität weitgehend unbestritten ist und die man dem Ausländer zum Gebrauch empfehlen kann. Trotzdem kann nicht ausgeschlossen werden, daß die notwendige subjektivität der Bearbeiter in Einzelfällen zu Entscheldungen gefuhrt hat, die für diesen oder jenen Benutzex unverständlich sind.

Für Hinweise und Kritik danken wir den Mitgliedern eines Fachausschusses des Deutschen Volkshochschulverbandes, L. Götze (München), H.P. Kromann (Kopenhagen), R. Schäpers (Ismaning), die eine frühere Fassung dieses wörterbuchs begutachtet haben. Für zahlreiche Anregungen danken wir auch M. Dordevíc (Sarajevo), B. Engelen (Münster), J. Juhász (Budapest), M. Kaempfert (Bonn), O. Leirbukt (Troms $\phi$ ), K. Tarvainen (JYväskylä) und anderen.

Wir hoffen, daß trotz aller Fehler und Unzulänglichkeiten dieses Buch seiner Zielsetzung gerecht werden kann. Für weitere kritische Anmerkungen sind wir jederzeit dankbar.

Ulrich Enge1

Helmut Schumacher 


\section{EINLEITUNG}

Angelika BALLWEG-SCHRAMM - Ulrich ENGEL -

Sabine PAPE - Helmut SCHUMACHER

o. Vorbemerkungen

1. Zielsetzung

1.1. Anwendungsgebiete

1.2. Zielgruppe

1.3. Konzeption

2. Theoretische Grundlage

2.1. Konnexion: Konstituenz und Dependenz

2.2. Valenz

2.3. Verbgrammatik

2.4. Satzglieder: Ergänzungen und Angaben

2.5. Klassifikation der Ergänzungen

2.6. Satzmuster und Satzbauplan

2.7. Beschreibungsebene

3. Aufbau des Lexikons

3.1. Verben und Wörterbucheinheiten

3.2. Aufbau der Lexikonartikel
4. Verbalkomplex

4.1. Bestandteile des Verbalkomplexes

4.2. Präfixverben und Verben mit Verbzusatz

4.3. Lexikalische Fügungen

4.4. Reflexivverben

4.5. Nicht kommutierbares es

4.6. Elliptische Sätze

4.7. Klassifizierung verbabhängiger infinitivischer Verbalsyntagmen

4.8. Komplexe Prädikate

5. Ergänzungsklassen 52

5.1. E

5.2. $\mathrm{E}_{1}$

5.3. $\mathrm{E}_{2}$

5.4. $\mathrm{E}_{3}$

5.5. $\mathrm{E}_{4}$

5.6. $E_{5}$ und $E_{6}$

5.7. $E_{7}$ und $E_{8}$

5.8. $\mathrm{E}_{9}$ 
6. Satzförmige Ergänzungen

6.1. Definition der SE

6.2. SE-Klasse

6.3. SE-TYP

6.4. SE-Form

7. Passivfähigkeit

7.1. Formen des Passivs

7.2. Angaben zur Passivfähigkeit

7.3. Volles Passiv

7.4. Unpersönliches Passiv

8. Beispielsätze und Bemerkiungen

8.1. Problematik der Beispiele

8.2. Form der Beispiele

8.3. Arten von Beispielsätzen

8.4. Codierung der Beispielsātze

8.5. Bemerkungen im wörterbuch

9. Maschinenausdrucke zu Valenzlexikon und Valenzregister

9.1. Valenzlexikon

9.2. Valenzregister
0. Vorbemerkungen

0.1. Das vorliegende "Kleine Valenzlexikon deutscher Verben" (im folgenden KVL genannt) umfaBt 461 der gebräuchlichsten Verben und gibt Informationen über deren morphosyntaktische Verwendung.

Im Rahmen des Forschungsunternehmens "Grundstrukturen der deutschen Sprache" 1 wurde 1970 am Institut fủr deutsche Sprache in Mannheim mit der systematischen Beschreibung der Valenzen deutscher Verben begonnen, nachdem die theoretischen Grundlagen einer hierfür geeigneten Verbvalenzgrammatik weitgehend erarbeitet waren. ${ }^{2}$ Das Lexikon wurde von einer Arbeitsgruppe aus wissenschaftlichen'und studentischen Mitarbeitern unter Leitung von Ulrich ENGEL und Helmut SCHUMACHER verfaßt. Dabei zeigte es sich, daß die theoretische Basis für eine adä-

1 Vgl. Engel, Grundstrukturen.

2 Vgl. Engel, Deutsche Satzbaupläne; ders., Satzbaupläne und ders., Thesen. 
quate Darstellung noch nicht ausreichte und daher in zahlreichen Einzelpunkten modifiziert oder erweltert werden muBte. Man kann somft feststellen, daß die Wörterbuchbeschreibung in nicht unerheblichem MaBe zur Verbesserung der Theorle beigetragen hat. Ein Teil der in diesem zusammenhang entstandenen Einzelstudien ist in elnem parallel erschelnenden Forschungsbericht zusammengefaßt. 1

An weiteren Mannheimer Arbeiten zur Verbvalenz, die auf dem gletchen oder nur geringfigig modifizierten theoretischen Ansatz bexuhen, sind die Monographie von Beruhard ENGELEN über Satzbauplan und Wortfeld ${ }^{2}$ sowle die tn Kürze erscheinende Syntax von ulrich ENGEL $^{3}$ zu nennen, die sich mit diesem Lexikon wechselseitig beeinfluBt haben. Elne zusammenfassende Dar-

Vg1. Schumacher (Hrsg.), Untersuchungen.

2 Vgl. Engelen, Untersuchungen.

3 Vgl. Engel, Syntax. stellung verschiedener Ansätze der Valenztheorte und Dependenzgrammatik von Helmut SCHUMACHER ist ebenfalls in Vorbereltung. ${ }^{4}$ Daher kann hier auf eine Auseinandersetzung mit verwandten Theorien weitgehend verzichtet werden.

0.2. Das vorlfegende Lexikon ist als Teil eines größeren Arbeltsvorhabens zu verstehen, bet dem ca. 1500 deutsche Verben in analoger Weise beschrieben werden sollen. Dieses "Erweiterte Valenzlexikon" (EVL) wird etwa $1976 / 77$ abgeschlossen sein. Auf die Gründe für die hier getroffene Auswahl der Vexben wird $1 \mathrm{~m}$ folgenden Kapitel eingegangen.

Bei der Erarbeitung des Lextkons wurde so vorgegangen, daß von den Bearbeitern die vorgegebenen Verben versuchsweise mit allen klassen dex morphosyntaktischen Umgebung komblniert wurden, um die bet den einzelnen

4 Vgl. Schumacher, Valenz und Dependenz. 
Verben möglichen strukturen herauszufinden. Auf die bei diesem Verfahren entstehenden Probleme kann hier nicht näher eingegangen werden. Eine ausführliche Diskussion findet sich im Forschungsbericht in der Einleitung von Helmut SCHUMACHER ${ }^{1}$ und im Beitrag von Bernd Ulrich BIERE. ${ }^{2}$

Es ist noch $\mathrm{zu}$ exwähnen, daß die Kompetenzentscheidungen der Mitarbeiter natürlich durch die Konsultation der einschlägigen neuen Wörterbücher, in denen Verbumgebungen aufgeführt werden, abgesichert wurden. Darüber hinaus dienten auch die Ergebnisse eines parallel zu diesem Arbeitsvorhaben entstandenen Valenzregisters als empirisches Korrektiv. Bei diesem Unternehmen wird eine Textauswahl aus dem "Mannheimer Corpus" nach den vorhandenen Satzmodellen analysiert sowie eine Anzahl von weiteren morphosyntaktischen. Informationen $\mathrm{zu}$ den

1 Vg1. Schumacher, Zum Forschungsbericht, S. 10-13. 2 Vgl. Biere, Ergãnzungen, S. 129-133. untersuchten Texten gegeben. ${ }^{3}$

\section{1. $\mathrm{Z}$ i e Isetzung}

1.1. Anwendungsgebiete

Dieses Valenzlexikon verfolgt das Ziel, die möglichen Verwendungsweisen deutscher Verben systematisch darzustellen. Die im Lexikon verzeichneten strukturen können als wesentlicher Teil der für die Bildung von morphosyntaktisch korrekten Sätzen notwendigen Produktionsregeln verstanden werden.

Es ist allgemein bekannt, daß besonders dem deutschlernenden Ausländer oft Fehler der Art unterlaufen, $\mathrm{da} \beta$ er Verben nicht mit der von ihnen geforderten strukturellen Umgebung kombiniert. Man findet in Schülerarbeiten häufig Fehler des Typs: ${ }^{4}$

3 Vgl. dazu Schumacher/Höfert, Erläuterungen. Dieses Projekt wird voraussichtlich im Laufe des Jahres 1977 abgeschlossen werden.

4 Die Fehlerbeispiele stammen aus den Untersuchungen von J. Kubczak, IdS Mannheim. 
*Das persönliche Interesse ist kaum zu dem Einfluß zu trennen.

"Der Lehrer' hat sich mehrfach auf sprachliche Erscheinungen gestoßen.

"Verlaßt euch nie filr andere!

Daher ist es eln wtchtiges ziel von Valenzwörterbüchern, gerade im Unterrichtsfach Deutsch als Fremdsprache als Hilfsmittel zu dienen, un die Blidung von morphostrukturell korrekten Sätzen zu gewährleisten.

Ein solches Valenzlexikon kann auch. eine wichtige Grundlage bei der Erstellung von kontrastiven Grammatiken bilden. Die hier verwendeten Beschreibungskategorien sind zwar nur auf eine Sprache bezogen, jedoch ist es möglich, das system so zu erweltern, daß die Ubereinstimungen und Unterschiede in der Ungebung entsprechender Verben zweier Sprachen dargestellt werden können. Allerdings kann man dabei kaum auf eine explizite semantische Beschreibung verzichten, da es nur sinnvoll ist, Ausdrucke zu kontrastieren, die inhaltitch einander entsprechen.

Ein anderes wichtiges Anwendungsgebiet ist der Bereich der maschinellen syntaktischen Analyse. In der Regel werden bei diesen Unternehmungen unfangreiche Lexika gespeichert, die u.a. bei den Verben die zugehörigen Umgebungen erfassen. Die Informationen dieses Lextkons sind bel einem Ids-Projekt dieser Art zugrundegelegt worden. ${ }^{1}$

\subsection{Zielgruppe}

Das KVL ist in erster Linie als ein HAlfsmittel fur den Deutschunterricht gedacht. Es gehört zum Begleitmaterial des zertifikats "Deutsch als Fremdsprache", das vom Deutschen Volkshochschulverband und vom Goethe-Institut entwickelt wurde. ${ }^{2}$

Bei der Prüfung zux Exlangung des Zertifi-

1 Vgl. Arbeitsgruppe MasA, Syntaxanalyse I und dies., Syntaxanalyse II.

2 Vgl. Steger (Hrsg.), Zertifikat 'DaF' und Brosch, zextifikat DaF, S. 149-152. 
kats muß der Kandidat den Nachweis exbringen, daB er uber Grundkenntnisse der deutschen Sprache verfügt, die es ihm erlauben, sich in Alltagssituationen sprachlich zu behaupten und einfache Sachverhalte mündlich oder schriftlich zu formulieren. ${ }^{1}$ Die Prüfungstests stützen sich auf eine Liste von grammatischen strukturen und eine Wortliste, deren Beherrschung vorausgesetzt wird.

Dieses Lexikon umfaBt genau die Verben die in der Wortliste zum Zertifikat enthalten sind. Die Kriterien für die Ermittlung des Mindestwortschatzes im Bereich der Verben folgen somit denen der Wortliste und unterliegen der damit verbundenen Problematik. Die Wortilste umfaßt etwa 2000 Items, die aus einer Kompilation der bis 1970 vorhandenen Basiswortschatzsammlungen gewonnen wurden. Die kompilierte Liste wurde von einem Fachausschuß aus Theoretikern und

1 Vgl. Steger (Hrsg.), Zertifikat 'DaF', S. 7.
Praktikern revidiert und in mehreren Arbeitsgängen auf Konsistenz und Angemessenheit für die wichtigsten Redekonstellationen der zielgruppe hin uberprüft. ${ }^{2}$ Die Schwierigkeiten liegen u.a. darin, daß die Zielgruppe für das Zertifikat im In- und Ausland nicht einheitlich ist und somit auch die möglichen Kommunikationssituationen sehr verschieden sein können. ${ }^{3}$ Außerdem fehlte es noch weitgehend an einer exakten Bestimmung der Lernziele, zu deren Festlegung in der Zwischenzeit erste Schritte unternommen wurden. ${ }^{4}$ Es ist sicher, daB diese Uberlegungen in absehbarer Zeit $\mathrm{zu}$ einer Modifikation der Wortliste führen werden, der in zukünftigen Fassungen des KVL Rechnung getragen werden soll, soweit der Bereich der Verben betroffen ist. Auf die Be-

Vg1. ebd., S. 15-16.

3 Vgl. ebd., S. 13-14,

4 Vgl. Pädagogische Arbeitsstelle des DVV, Erster Zwischenbericht und Baltruweit, Analyse. 
ziehungen zwischen der Wortliste und den Belsptelsätzen wird in 8.1. näher eingegangen.

Naturlich ist der Gebrauch dieses Lexikons nicht auf die Vorbereftung zur zertifikatsprüfung allein beschränkt. Es wendet sich vielmehr an alle, die mit dem Deutschunterrlcht auf der Grundstufe befaBt sind.

\subsection{Konzeption}

Wie schon gesagt beschränkt sich dieses Lexikon auf die moxphosyntaktische Ebene unter Verzicht auf phonetische und morphologische Ingaben $z u$ den Verben, da diese leicht in verschledenen anderen Wörterbüchern nachgeschlagen werden kömnen.

Vexzichtet wurde auch auf eine explizite rinbezlehung der Semantik, da der damalige Forschungsstand elne adäquate semantosyntaktische Beschretbung noch nicht exlaubte. Naturlich wäre es möglich gewesen, im slnne der Selektionsregeln der Generativen Trans- formationsgrammatik Besetzungsrestriktionen für die elnzelnen posittonen in dex Umgebung der Verben $\mathrm{zu}$ formulferen. Es hat sich jedoch gezelgt, daB Informationen die-. ser Art nur wenig zur Lösung der Probleme $1 \mathrm{~m}$ Auslănderunterricht beitragen. Man kann davon ausgehen, daß jeder, der die Bedeutung eines Verbs wie denken kennt, die subjektstelle mit einem Element besetzen wird, dessen Denotat ein Merkmal wie 'Intentional handelnd' zukommt. Viel wichtiger ist dagegen eine Analyse dex Verbinhalte und der Relation zwischen den Inhaltskomponenten und der Umgebung der Verben. An einem solchen Projekt wird $z$. zt. am Ids gearbeltet. ${ }^{1}$ Innerhalb der hier zugrundegelegten Theorie, die im folgenden Kapitel erläutert wird, haben wix allexdings eine gewisse Vollständigkeft in der Beschreibung angestrebt. Es wird versucht, alle Daten zum Verb und seiner Um-

1 Vgl. Projektgruppe Verbvalenz, Jahresbericht 1975. 
gebung anzugeben, die für bestimmte Verbgruppen spezifisch sind.

\section{Theoretische Grundlage}

2.0. Das Kleine Valenzlexikon ist erarbeitet und formuliert worden im Rahmen einer Dependenz-Verb-Grammatik. ${ }^{1}$ Wenn sich der Benutzer über die hier verwendeten und im folgenden ausfuhrlich beschriebenen Kategorien Klarheit verschafft, wird er aus dem Lexikon die gewïnschten Informationen entnehmen können, auch wenn er sich einer ganz anderen linguistischen Richtung zugehörig fühlt.

2.1. Konnexion: Konstituenz und Dependenz Jede Grammatik enthält einen Teil, in dem relativ elementare strukturen erzeugt werden: Wortgruppen, Satzglieder, Sätze usw. Dieser Teil, der die primäre Kombinierbarkeit von Elementen regelt, wird hier

1 Vgl. dazu Engel, Bemerkungen.

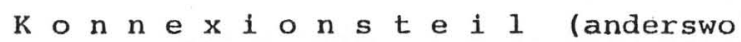
Basisteil o.ä.) genannt. Jede Grammatik, ganz gleich wie sie sich nennt, enthält darủberhinaus einen Transformationsteil, der neue Konstrukte aus den zuvor erzeugten Konstruktionen ableitet; Transformationen sind unverzichtbar, weil eine Grammatik ohne sie alle Dimensionen sprengen würde. Die Abgrenzung zwischen Konnexionen und Transformationen kann man sich folgendermaßen deutlich machen. Verben wie beweisen können mit einem im Nominativ ("Subjekt"), einem Element im Akkusativ und einem (fakultativen) Element im Dativ verbunden werden; sie können außerdém a1le Verben mit Orts-, Zeit- und Modalbestimmungen (z.B. dort, heute, schnell) sowie mit Negationen (z.B. nicht) kombiniert werden. Diese reine Kombinierbarkeit wird im Konnexionsteil geregelt. Daß aber auf Grund solcher Kombinatorik zuletzt Sätze wie Ich habe ihm das heute schnell bewiesen. Dem habe ich das gesterm schnell bewiesen. 
Beweise ihm das doch schnel.z.

Hast du es ilm nicht bewiesen?

u.a. erzeugt werden könnén, bedarf zusätz1icher Transformationen. Das Valenzlexikon liefert im wesentlichen Informationen uber jène primăxe Kombinierbarkeit, die In den konnexionste11 gehört.

Natürlich können Konnexionsregeln nicht beliebig formuliert werden; jedermann weiß, daß nicht aile Elemente miteinander kombiniert werden können. Zum Beispiel kann helfen nicht mit einem Element tm Akkusativ, wohl abei mit einem solchen im Dativ verbunden werden: Ich helfe meinem Bruder. Alle wörter sind mit Merkmalen ausgestattet, die thre Kombinierbarkeit steuern. Einen erheblichen Tell dieser kombinatorischen Merkmale enthält das Valenzlexikon; es ist damit dem Konnexionsteil dex Grammatik umittelbar zugeordnet.

Kommexionen können grundsätzlich auf zwei Arten dargestellt ("organisiert") werden: konstituenziel1 oder dependenziel1. Das Prinzip dex $k$ o $n$ s $t \cdot t u$ e $n z$ beruht auf der Teil-Ganzes-Beziehung. Den Satz Mein alter Nachbar hilft meinem Brudex. etwa beschreibt die Konstituentengramutik mit folgenden Aussagen:

Der Satz besteht aus 2 Teilen (Subjekt und prädikát).

Das Subjekt besteht aus Artikel, Adjektiv, Nomen usw.

Umgekehrt kann formuliert werden:

Artikel, Adjektiv, Nomen bilden zusammen das subjekt.

Subjekt und Prädikat bilden zusammen den Satz S.

Das Prinzip der $D$ e $p$ e $n d e n z$, wie es hier verstanden wird, greift ausschließlich auf die Teile zurïck und ordnet sie auf Grund threr kombjnatorischen Merkmale, wobei bestimuten Elementen eine höhere Position gegenuber anderen Elementen zugewiesen wird; man sagt, das Nomen $x$ e g $i$ e $r$ e 
Artikel und Adjektiv oder "Possessivpronomen", das Verb ( helfen) seinerseits regiere zwei Nomina, eines im Nominativ, eines im Dativ; umgekehrt: Die Nomina hängen vom Verb ab, Artikel/Adjektiv/"Possessivpronomen" hängen vom Nomen $a b$.

Stellt man dies zeichnerisch dar, so entstehen äußerlich ähnliche Baumdiagramme, die aber in Wirklichkeit ganz Verschiedenes aussagen. Das konstituenzielle Diagramm zu dem Saț Der alte Nachbar hilft seinem Bruder. hat, nach verbreitetem Verfahren, folgende Form:

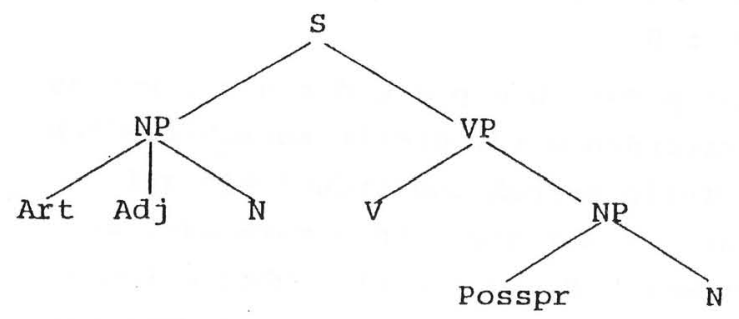

Das entsprechende dependenzielle Diagramm:

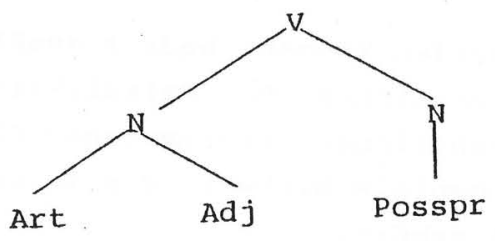

Das dependenzielle Diagramm hat, wie man sieht, keine "Zwischenkategorien", jedes Element ist nur einmal repräsentiert, die Summe der Symbole ergibt genau die Satzstruktur (während die Summe der konstituenziellen Symbole ein Mehrfaches der Satzstruktur ergibt).

Es muß nun verständlich sein, daß im Rahmen einer dependenziellen Darstellung die regierenden Elemente $\mathrm{R}$ e $\mathrm{g}$ e $\mathrm{n} s$ (Plural: Regentien) heißen, die abhängigen regierten Elemente $D$ e $p$ e $n$ d e $n$ s (Plural: Dependentien). Dabei gilt die Regelung, daß ein Element (wenigstens prinzipiell) mehrere Dependentien, aber immer nur ein 
Regens haben kann. Das heibt: Verzweigungen gibt es immer nur von oben nach unten.

2.2. Valenz

Oben war von den kombinatorischen Merkmalen die Rede, die den wörtern zukommen. Im Grund ist damit genau das gemeint, was in der traditionellen (und noch in vielerlei Ausprägungen der neuesten) Grammatik als $R$ e $k$ $t i o n$ bezeichnet wird. Rektion gibt an, welche Elemente ein wort regieren kann (diese "dependenzlelle" Formulierung láßt sich, wenn auch mit etwas Mühe, durchaus auch "konstituenzie11" ausdrüken). Rektion ist jedoch ein sehr welter Begriff. Er kann für ganze Wortklassen (Wortarten) gelten: jedes Jomen kann ein Genitivattribut regieren: der Schatten des Körper's; der Körper des Kutschers; der Kutscher des Grafen usw. Er kann auch nur für Teile von Wortklassen verwendet werden; manche Nomja können auch etn präpositionales ntribut zu sich nehmen: Die Hoffnung auf bessere Reiten.
Fur diesen letzteren, 1inguistisch interessantexen und pädagogisch schwierigeren Fall verwenden wir den Begriff der $V$ a 1 e $n z$. Damit wird Valenz als Sonderfall der Rektion definiert: Valenz ist die Rektion von Teilen von Wortklassen. Urspringlich stammt der Begriff der Valenz aus der Chemie. Er wird dort, gleichbedeutend mit "Wertigkeit", gebraucht für die Fähigkeit chemischer Elemente, sich mit anderen chenischen Elementen $z u$ verbinden. In dex Iinguistik wurde der V́alenzbegriff durch TESNIERE bekanntgemacht. Zwar spricht TESNIERE ausdrücklich nur von der $z$ a h 1 der Aktanten, die durch die Valenz festgelegt werde. 'Aus zahlreichen Bemerkungen in seinem Hauptwerk geht jedoch hervor, daß er den Valenzbegriff auf $\mathrm{zahl}$ und Art der dell Verb zugeordneten Elemente - der Aktanten - bezieht. ${ }^{2}$ Da Valenz somit nur für Subklassen von wort-

1 Vgl. Tesnière, Eléments, S. 238.

2 Vg1.Tesnière, Eléments, z.B. S. 106, 108, 242 et passim. 
klassen gilt, während Rektion daneben auch ganze Wortklassen kennzeichnen kann, ist die Valenz als ein Sonderfall der Rektion anzusehen. Diesen subklassenspezifischen Valenzbegriff haben nicht nur BRINKMANN, ${ }^{1}$ GRE$\mathrm{BE},{ }^{2}$ HERINGER ${ }^{3}$ in die deutsche Grammatik ubernommen; er gilt vielmehr faktisch für die meisten Darstellungen, die sich überhaupt des Valenzbegriffs bedienen.

Anders verfährt HELBIG, der - in allzu enger Auslegung TESNIEREs - den Valenzbegriff auf das Quantitative beschränkt: Valenz bestimmt lediglich, wi e v $i$ e 1 e Aktanten/Ergänzungen/Mitspieler etwa ein Verb hat oder haben kann; fur die Art dieser Aktanten benötigt HELBIG darum den zusätzlichen (vom klassischen strukturalismus übernommenen und wiederum willkürlich eingeengten) Begriff der Distribution. ${ }^{4}$

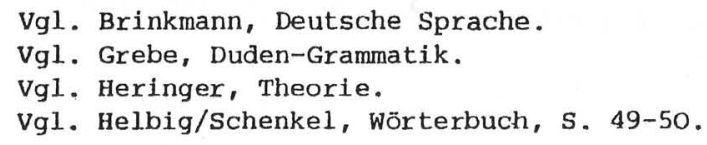

Ein weiterer Valenzbegriff, der, TESNIEREs Intentionen entsprechend, auch im Kleinen Valenzlexikon verwendet wird, vermag das gleiche zu leisten wie das Begriffspaar im "Wörterbuch zur Valenz und Distribution deutscher Verben".

Auf der anderen Seite verwenden wir - mit HELBIG und anderen Leipziger Linguisten - den Valenzbegriff nicht nur im Hinblick auf das Verb. Wenn Valenz als Rektion von Teilen von Wortklassen definiert wird, gilt sie prinzipiell für beliebige Wortklassen. Valenz ist dann (ebenso wie Rektion) als eine bestimmte Art von Vorkommensrestriktion ein universeller Relationsbegriff, der auch auf sprachen Anwendung finden kann, die gar keine Verben haben. Was von Einzelsprache zu Einzelsprache wechselt, sind die Elemente (Wörter) und ihre Klassifikation. Aber es kann keine natürliche sprache geben, in der Vorkommensrestriktionen nicht mit dem Begriff der Valenz darstellbar wären.

5.Vgl. z.B. Schenkel, Valenz; Sommerfeldt/Schreiber, Untersuchungen; dies., Wörterbuch; dies., $\mathrm{zu}$ einem wörterbuch. 
Auf die deutsche sprache bezogen, kann Valenz mindestens auch dem Nomen und dem ndjektiv zugeschrieben werden (mögl1cherwelse noch weiteren Wortklassen).

Einem verbreiteten Mißverständnis soll gleich hier begegnet werden. Die Tatsache, daß man nicht *Er brach lange zusammen. sagen kann, daß das Wort lange nicht mit Verben mit punktue 1ler Bedeutung vorkommt, daß es somit nur mit einem Teil der Wortklassen kombiniert werden kann, darf nicht zu dem Schluß verfuhren lange sei. deshalb valenzbedjngt. Vorkommensrelationen, somft auch Valenzrelationen, bestehen in der Grammatik immer zwischen Klassen von Elementen (sogenannten Kategorien). Die Kategorie, der lange angehört, ist die Temporalbestimmung. Es kann nicht bezweifelt werden, daß jedem deutschen Verb eine Temporalbestinmung zugeordnet werden kann. Freilich hängt die Frage, was im Eillzelfall valenzbedingt sei, von der Kategorienbildung ab; diese ist grundsätzlich ins Ermessen des Grammatikers geste 11 t.
2.3. Verbgranumatik

In vielen Lexika der linguistischen Terminologie steht unter dem stichwort "Dependenzgrammatik", daß es sich hierbei um Gramatiken handle, bei denen das Verb als strukturelles Zentrum des Satzes angesehen werde. Das ist wissenschaftshistorisch eintgermaßen richtig, theoretisch jedoch unhaltbar. Eine Gramnatik mit dependenziellem Konnexionsteil (vielfach kuxzerhand als "Dependenzgrammatik" bezeichnet) ordnet ihre Kategorien nach Abhängigkeitsrelationen. Dabei bleibt zunächst völlig offen, was zentral und was peripher, was regierend und was abhängig ist. Die wôrterbuchmacher haben in ihrem $\mathrm{Be}-$ mïhen, die "Dependenzgramatik" grgen die "Konstituentengrammatik" abzugrenzen, unmerk11ch ein anderes Begriffspar abernommen, das man mit "Verbgrammatik" gegen "subjektPrädikat-Grammatik" bezelchnen könnte. 1

1 Vgl. Engel, Bemerkungen, S. 137-141. 
Verbgrammatik ist eine Grammatik, die in ihrem Konnexionsteil dem Verb eine besondere (zentrale, regierende oder wie immer man will) Rolle zuweist; eine solche Grammatik liegt dem Valenzlexikon zugrunde. Für die Wahl einer solchen Grammatik gibt es mancherlei Argumente; das triftigste ist, daß das Verb im (deutschen) satz durchschnittlich die reichsten und vielfältigsten Verbindungen eingeht. Die Subjekt-PrädikatGrammatik teilt jeden Satz in einem ersten Schritt in zwei Teile, die "Subjekt" und "Prädikat" (häufig auch anders) genannt werden. Damit ist klargestellt, daß dem Verb in dieser Grammatik keine irgendwie hervorgehobene Rolle zukommen kann. Diese SubjektPrädikat-Grammatik wird, auch wenn sie eine uralte Tradition hat, für unsere Darstellung nicht verwendet. Die zweigliederung in ein "Genanntes" und ein über dieses Genannte "Ausgesagtes" ist zwar in Äußerungen/Aussagen sinnvoll, aber Äußerungen und Sätze sind begrifflich prinzipiell verschieden, auch wenn sie häufig dieselbe. Ausdrucksstruktur haben. Die Darstellung des Kleinen Valenzlexikons bewegt sich strikt im Rahmen der Satzgrammatik, in der andere strukturen zu gelten haben.

Zur Ehrenrettung der Verfasser linguistischer Fachwörterbücher muß gesagt werden, daß die melsten bisher ausgearbeiteten Dependenzgrammatiken zugleich Verbgrammatiken sind (eine wichtige Ausnahme bildet HERINGER), und daß wahrscheinlich alle derzeit existierenden Konstituentengrammatiken zugleich Subjekt-Prädikat-Grammatiken sind. Aber diese weitgehende Koinzidenz erlaubt nicht eine Gleichsetzung der beiden Dichotomien.

\subsection{Satzglieder: Ergänzungen und Angaben}

Satzglieder sind die Elemente, die zusammen mit dem Verbalkomplex, und zwar in Abhängigkeit vom Verb, den Satz konstituieren.

\section{Dem Satz}

Die ganze Geschichte erfahren wir wahrscheinlich heute. 
kann die (vereinfachte) Dependenzstruktur

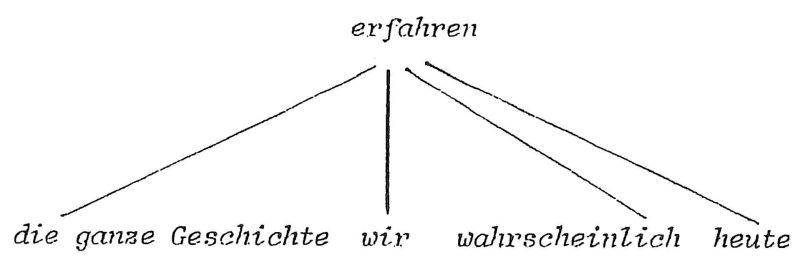

zugeordnet werden.

Fis ist offenkundig, daB die viex satzglieder in teilweise ganz unterschiedlichen Bedeutungsbeziehungen zum Verb erfalien stehen. nber Dependenzrelationen sind grundsätzlich als Vorkommensrelationen, Zuordnungsrelationen festgelegt; sie sind damit nicht notwendig semantische Relationen. Da alle vier Elemente mit Verben wie erfahren kombinierbar sind, ist es legitim (wennglefch nicht unumgäng1tch), exfahren als Regens und die vier Elemente als $D$ e p e $n$ de $n t i$ e $n$ des Verbs, mithin als satzglieder, aufzufassen.

Können so, wenigstens auf der hier gewählten Beschreibungsebene, semantisch unterschiedliche Relationen nicht bericksichtigt werden, so muß doch ein anderer unterschied beachtet werden: zwei dex Satzglieder (die belden ersten) sind valenzbedingt, die anderen belden (die existimatorische Bestimmung walurscheintich und die zeitbestinumung heute) hingegen sind nur durch allgemeine Rektionen vom verb abhängig. Auf diese Weise unterscheiden wir Ergänzungen und $\lambda$ ngaben: $E$ r g ä $n z u n-$ $g$ e $n$ sind Glieder, die valenzbedingt vom Verb abhängen, die somit nur mit einem Teil der Wortklasse Verb kombinierbar sind; Angaben !ningegen sind mit beliebigen Verben kombinierbar.

Mit diesen Definitionen ist der streit um die Abgrenzung zwischen Ergänzungen und 
Angaben freilich höchstens auf einer sehr abstrakten Ebene entschieden; in der Praxis bleiben nach viele Schwierigkeiten bestehen. Merkwürdig ist dabei nur, daß bei den Linguisten eigentlich weniger die Sache selbst zur Diskussion stand (dariber, was Ergänzung, was Angabe sei, ist man sich weithin einig) als explizite Definitionen. ${ }^{1}$ Alle Versuche, die Ergänzung als "notwendig", "strukture11 notwendig", "obligatorisch" u.ä. zu definieren, wurden zwangsläufig ad absurdum geführt, weil jeder Autor zugestehen mußte, daß es eben auch fakultative Ergänzungen gebe. Auch andere Bemühungen helfen nicht viel weiter, so der Versuch, Ergänzungen und Angaben auf Unterschiede in der "Tiefenstruktur" zurückzuführen. Und HELBIGs Versuch, die Ersetzbarkeit durch Nebensätze (Gliedsätze) als distinktives Merkmal für Angaben anzusetzen, befriedigt deshalb nicht, weil vie-

1 Einen Uberblick über die bisher vorgelegten Lösungsvorschläge gibt Biere, Ergänzungen. le Angaben - übrigens auch ein Teil der von HELBIG angeführten - überhaupt nicht in Satzform möglich sind. ${ }^{2}$ Das hier vorgeschlagene Verfahren, das in erster Linie auf zuordnungsmöglichkeiten beruht, ist jedenfalls im Rahmen der sprachlichen Kompetenz überprüfbar. So bleibt für die Zweiteilung der Satzglieder nur das oben geschilderte Kriterium der Art der Abhängigkeit. Will man diesen Unterschied auch graphisch darstellen, so kann man die Dependenzstriche bei Angaben mit einem $x$ versehen; dies soll markieren, daß der Bereich des Regens größer ist als angegeben. Das Diagramm erhielte dann, immer noch vereinfacht, die folgende präzisere Form

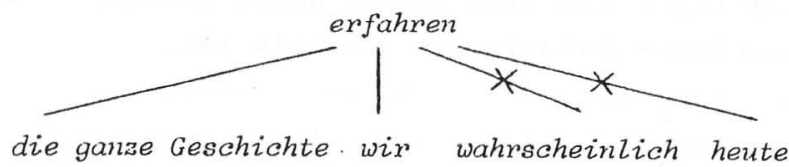

2 Vgl. Helbig, Valenz und Tiefenstruktur, S. 164-166; ders., Valenz, S. 25-34; Helbig/ Schenke1, Wörterbuch, S. 35-40. 


\subsection{Klassifikation der Ergänzungen}

Die Gramutik kann sich mit einzelnen Elementen nur insofern beschäftigen, als sie bestimmten K.1assen angehören; nur mit Hilfe der Element-Klasse-Relation, die Aussagen wie "a ist ein (Element der klasse) A" ermöclicht, jst ibersicht1iche sprachbeschreibung möglich. Bei. der Klassenbildung für Satzglieder, also auch für Ergänzungen, spielt das $\mathrm{p}$ a $\mathrm{r}$ a d $i \mathrm{~g} \mathrm{ml}$ a eine grundlegende Rolle. Als Paxadigma hat die Klasse der Elemente zu gelten, die im selben Kontext austauschbar sind; zum Beispiel

$$
\text { Bruno weiB }\left\{\begin{array}{l}
\text { es } \\
\text { das } \\
\text { niehts } \\
\text { die ganze Geschinhte } \\
\text {, was Franen winschen } \\
\text { usw. }
\end{array}\right\} \text {. }
$$

Der Kontext ist dabei kategorial bestimnt, hier etwa als "Nominalgruppe im Nominativ"
(Bruno ), "finites Verb" ( weiß). Kategorien sind frelitch ule vorgegeben, sondern werden vom Grammat1ker definiert. Verändert sich die kategorlale Wiedergabe des Kontextes, so muß sich auch das paradigma ändern. Dies ist zumindest.efner der Gründe dafür, daß so wenig̣ Einigkelt uber die klassifikation der Ergänzungen besteht. Die Tabelle auf S. 22/ 23 bietet eine Synopse der.w1chtigsten Klassifikationen für Exgänzungen. Dabei findet man in spaite 1 eine Notation für Ergänzungen, wie siẻ auf Seite 26 erläutert ist. Zur angefühxten $T$ e $r m i n \circ 1 \circ g i$ e ist freilich zu sagen, daß dieselbe Bezelchnung bei verschiedenen Autoren oft für Verschiedenes steht; so verstehen keineswegs alle Gramutiker unter "Akkusativobjekt", "Präpositionalobjekt" usw. dasselbe. Die Tabe1le versucht jedoch, ausgehend von den im KVL verwendeten Kategorien, nach Möglichkejt jeweils b e g r i f $\mathrm{f} 1 \mathrm{i}$ c h G 1 e i $c h$ e $s$ nebeneinanderzustelleri. 


\begin{tabular}{|c|c|c|c|c|}
\hline & $\begin{array}{l}\text { ERBEN, } \\
\text { Grammatik }\end{array}$ & $\begin{array}{l}\text { FLÄMIG, } \\
\text { Skizze }\end{array}$ & $\begin{array}{l}\text { GLINZ, } \\
\text { Innere Form }\end{array}$ & $\begin{array}{l}\text { GREBE, } \\
\text { Duden-Grammatik }\end{array}$ \\
\hline $\mathrm{E}_{\mathrm{o}}$ & $E_{1}$ & Subjekt & Grundgröße & Subjekt \\
\hline$E_{1}$ & $E_{4}$ & Akkusativobjekt & Zielgröße & Akkusativobjekt \\
\hline $\mathrm{E}_{2}$ & $\mathrm{E}_{2}$ & Genitivobjekt & Anteilgröße & Genitivobjekt \\
\hline $\mathrm{E}_{3}$ & $\mathrm{E}_{3}$ & Dativobjekt & Zuwendgröße & Dativobjekt \\
\hline$E_{4}$ & $\mathrm{E}_{5}$ & Präpositionalobjekt & & Präpositionalobjekt \\
\hline $\mathrm{E}_{5}$ & E & $\begin{array}{l}\text { Enge Adverbial- } \\
\text { bestimmung (lokal/ } \\
\text { instrumental/modal) }\end{array}$ & Lagegröße/ & $\begin{array}{l}\text { Raumergänzung } \\
\text { Zeitergänzung } \\
\text { Begründungsergänzung } \\
\text { teilw. Artergänzung }\end{array}$ \\
\hline $\mathrm{E}_{7}$ & $\mathrm{E}_{6}$ & $\begin{array}{l}\text { substantivisches } \\
\text { Prädikativ }\end{array}$ & Gleichgröße & $\begin{array}{l}\text { Gleichsetzungsnominativ } \\
\text { Gleichsetzungsakkusativ }\end{array}$ \\
\hline $\mathrm{E}_{8}$ & $\mathrm{E}_{\text {adj }}$ & $\begin{array}{l}\text { adjektivisches } \\
\text { Prädikativ }\end{array}$ & $\begin{array}{l}\text { Artangabe, } \\
\text { Lagegröße }\end{array}$ & Artergänzung \\
\hline $\mathrm{E}_{9}$ & & . & $\begin{array}{l}\text { abhängiger } \\
\text { Infinitiv }\end{array}$ & (Infinitiv) \\
\hline
\end{tabular}




\begin{tabular}{|c|c|c|c|c|}
\hline & $\begin{array}{l}\text { HELBIG/BUSCHA, } \\
\text { Grammatik }\end{array}$ & $\begin{array}{l}\text { IIERINGER, } \\
\text { Theorte }\end{array}$ & $\begin{array}{l}\text { KAUFMANN, } \\
\text { Grammatik }\end{array}$ & $\begin{array}{l}\text { SCHULZ/GRIESBACH, } \\
\text { Gramuatik }\end{array}$ \\
\hline$E_{O}$ & Subjekt & $\mathrm{E}_{1}$ & subjekt & $\begin{array}{l}\text { Subjekt } \\
\text { Prädikatssubjekt }\end{array}$ \\
\hline$E_{1}$ & Akkusativobjekt & $\mathrm{E}_{2}$ & Akkusativobjekt & $\begin{array}{l}\text { Akkusativobjekt } \\
\text { Prädikatsobjekt }\end{array}$ \\
\hline $\mathrm{E}_{2}$ & Genttivobjekt & $\mathrm{E}_{3}$ & Genittvobjekt & Genitivobjekt \\
\hline $\mathrm{E}_{3}$ & Dativobjekt & $\mathrm{E}_{4}$ & Dativobjekt & Dativobjekt \\
\hline $\mathrm{E}_{4}$ & Präpositionalobjekt & \multirow{3}{*}{$\mathrm{E}_{5}^{\prime}$} & Präpositionalobjekt & \multirow{3}{*}{$\begin{array}{l}\text { Lokal-r, Temporal- } \\
\text { Kausalergänzung }\end{array}$} \\
\hline $\mathrm{E}_{5}$ & \multirow[t]{2}{*}{ ndverbialbestimmung } & & $\begin{array}{l}\text { Raumergänzung } \\
\text { Zeitergänzung } \\
\text { Maßergänzung } \\
\text { Grundergänzung usw. }\end{array}$ & \\
\hline $\mathrm{F}_{6}$ & & & Richtungsergänzung & \\
\hline $\mathrm{E}_{7}$ & \multirow{2}{*}{$\begin{array}{l}\text { Subjektprädikativ } \\
\text { objektprädikativ }\end{array}$} & $\mathrm{E}_{6}$ & \multirow{2}{*}{ Artergänzung } & $\begin{array}{l}\text { Prädikatsnominativ } \\
\text { Prädikatsakkusat } 1 v\end{array}$ \\
\hline $\mathrm{E}_{8}$ & & $\begin{array}{l}\text { prädikatives } \\
\text { Adjekt } 1 v\end{array}$ & & Modalergänzung \\
\hline$E_{9}$ & $\begin{array}{l}\text { Infinitiv als lexikalisch- } \\
\text { idiomatischer prädikatsteil }\end{array}$ & $\begin{array}{l}\text { Infinitiv- } \\
\text { konstruktion }\end{array}$ & $\begin{array}{l}\text { Infinitiv als } \\
\text { Ergänzung }\end{array}$ & objektprädikat \\
\hline
\end{tabular}


Eine Klassifikation der Ergänzungen als Paradigmen im gleichen Kontext ist nicht völlig befriedigend, weil semantische Gesichtspunkte dabei weitgehend außer Betracht bleiben müssen; aber sie ist derzeit als einzige durchführbar. Alle Bemühungen, Ergänzungsklassen semantisch zu definieren (wie sie aus der GLINz-Schule als "zielgröße", "Zuordnungsgröße", "Anteilgröße", "Gleichgröße" u.a. bekannt sind), haben zu Irrtümern und gewaltsamen Umdeutungen geführt und sind mittlerweile. von ihren Urhebern wieder aufgegeben worden. 1

Jedoch selbst wenn man sich mit einer morphosyntaktischen Klassifikation im hier vorgeschlagenen Sinne begnügt, bleiben Probleme. Vielfach besteht Uneinigkeit darüber, was denn nun das wesentliche Merkmal elner Ergänzungsklasse sel; dies kommt

1 Vgl. Glinz, Innere Form, s. 4. auch in den oft unterschiedlichen Benennungen für gleiche oder ähnliche klassen (z.B. "Gleichgröße", "Gleichsetzungsnominativ", "substantivische Prädikatsnomen", "Subsumptivergänzung") zum Ausdruck. Hier kann die folgende Uberlegung helfen: Man kann jedes Paradigma, das einer Ergänzungsklasse entspricht, so ordnen, daß die möglichst konkreten Elemente am Anfang, die Elemente mit allgemeinerer Bedeutung aber am Schluß stehen. Dies spiegelt sich wieder in der Folge

am Tag, als der Regen kam

an jenem Tag

an dem Tag

damals

da

oder ähnlich in

der Mann, der Birnen verkauft

der besagte Mann

der Mann

der

er 
Die hier jeweils aufgelisteten nusdrücke bilden naturlich allein noch keine Ergänzungsklassen, können aber als Auszuge aus solchen aufgefaßt werden. Immer stehen bei. solcher Anordnung am Ende des Paradigmas Elemente mit geringer Eigenbedeutung, meist Pronomina oder Adverbien, deren semantische Hauptfunktion es ist, auf andere Textstellen zu verweisen. Mit Kar1 EUHLER ${ }^{1}$ und in Anlehnung an $L$. 'IESNIERE ${ }^{2}$ nennen wir diese Elemente $A \mathrm{n}$ a $\mathrm{p}$ e $\mathrm{r} n$. Sie können als "Leitformen" für die Klassifikation der Ergänzungen angesehen werden. Es lassen sich nämlich auf diese Art für das Deutsche 10 Ergänzungsklassen festlegen, die unter anderem an je spezifischen Anaphern kenntiich sind. Es folgt eine Liste dieser Ergänzungen. Die Kopfspalte enthält das Symbol, die zwelte spalte den gewäh1ten Terminus, die dritte spalte die Anapher, die vierte Spalte Beispielsätze, In

1 Vgl. Bühlex, sprachtheorie, S. 121 und S. 385 ff. 2 Vgl. Tesnière, Eléments, s. 89-91. denen die betreffende Ergänzung gesperrt gesetzt ist (sie kann durch die Anapher ersetzt werden).

Die hier gewählten Bezeichnungen werden durchgehend für das KVL verwendet. Sie stammen großenteils aus den Jahren 1971/1972, als dle Arbeiten am KVL begannen. Neuerdings werden verschiedent $11 \mathrm{ch}^{3}$ abweichende Termini verwendet.

Die Benennungen sind in manchen Fällen irreführend. So deckt die "Nominativergänzung" auch Nebensätze ab; unter Umständen ist es daher besser, nur die symbole zu verwenden. Bei der Anaphorisierung ergeben sich manchmal Probleme, die die zuordnung eines Elements zu einer bestimuten Ergänzungsklasse erschweren können. Darauf wird in Kapitel 5. näher eingegangen werden.

3 Z.B. Engel, Syntax. 


\begin{tabular}{|c|c|c|c|}
\hline $\mathrm{E}_{\mathrm{O}}$ & Nominativergänzung & $\begin{array}{l}\text { Personalpronomen } \\
\text { im Nominativ }\end{array}$ & $\begin{array}{l}\text { We } r \text { ihn } k \text { e } n n t \text {, sagt nichts. } \\
\text { Me } i n \text { B r u d e } r \text { sagt nichts. }\end{array}$ \\
\hline $\mathrm{E}_{1}$ & Akkusativergänzung & $\begin{array}{l}\text { Personalpronomen } \\
\text { im Akkusativ }\end{array}$ & Wir suchten $P i l z e$. \\
\hline $\mathrm{E}_{2}$ & Genitivergänzung & dessen, derer & 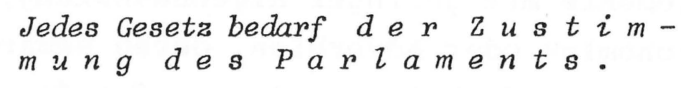 \\
\hline$E_{3}$ & Dativergänzung & $\begin{array}{l}\text { Personalpronomen } \\
\text { im Dativ }\end{array}$ & $\begin{array}{l}\text { Du solltest di e s e m Mann } \\
\text { nicht trauen. }\end{array}$ \\
\hline $\mathrm{E}_{4}$ & Präpositionalergänzung & $\begin{array}{l}\text { da(r) oder Personal- } \\
\text { pronomen mit nicht } \\
\text { austauschbarer Prä- } \\
\text { position (z.B. dazu, } \\
\text { zu ihm) }\end{array}$ & 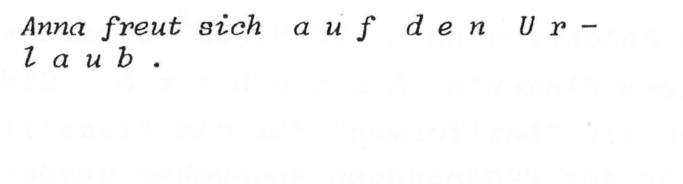 \\
\hline $\mathrm{E}_{5}$ & Situativergänzung & meist $d a$, dann & 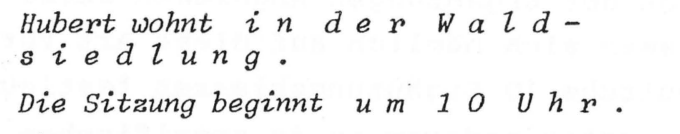 \\
\hline $\mathrm{E}_{6}$ & Direktivergänzung & $\begin{array}{l}\text { hin, dahin, von dort, } \\
\text { dorther }\end{array}$ & $\begin{array}{l}\text { Der Zug fährt } n a \propto h \text { Bonn. } \\
\text { Der Zug kommt a us Göt } t i n g \text { en. }\end{array}$ \\
\hline $\mathrm{E}_{7}$ & Einordnungsergänzung & es, so & $\begin{array}{l}\text { Mein Vetter heißt } H \text { e } i n \text { e } r \\
\text { Großvater ist } I n s p \text { e } k \text { t or. }\end{array}$ \\
\hline $\mathrm{E}_{8}$ & Artergänzung & es, so & $\begin{array}{l}\text { Man nennt ihn versehlagen. } \\
\text { Er ist krank. }\end{array}$ \\
\hline $\mathrm{E}_{9}$ & Ergänzungssatz & $\begin{array}{l}\text { (es ) geschehen/ } \\
\text { daß es geschieht }\end{array}$ & $\begin{array}{l}\text { Ich lasse } d i e k i n d e r \text { den } \\
T i s c h \text { ab r a } u m e n .\end{array}$ \\
\hline
\end{tabular}




\subsection{Satzmuster und Satzbaupläne}

Durch die Valenz jedes Verbs ist festgelegt, welche Ergänzungen in seiner Umgebung vorkommen können. Es ist nur eine überschaubare Anzahl solcher Kombinationen von Ergänzungen möglich. Diese Kombinationen nennen wir $s$ a $t z \mathrm{~m} u t e r$ (SM). Die wichtigsten sind in der Liste auf $S .28$ aufgeführt. Dabei entsprechen die zahlen den in 2.5. beschriebenen Indizes der Ergänzungen.

Man kann jedem Verbalsatz ein bestimmtes Satzmuster zuordnen. Allerdings liefern Satzmuster nur unvollkommene Beschreibungen. Sie sind auch für den Fremdsprachenuntericht allenfalls bedingt brauchbar. Es fehlt bei ihnen nämlich die schon oben angesprochene und in der Forschung vieldiskutierte Unterscheidung zwischen obligatorischen und fakultativen Ergänzungen. Dabei muß eine begriffliche Präzisierung vorgenommen werden: "obliga- torisch" darf nicht einfach gleich "notwendig". "fakultativ" nicht einfach gleich "weglaßbar" gesetzt werden. Man hat hier streng den kommunikativen vom (im engeren Sinne) grammatischen Bereich zu scheiden. Kommunikativ gesehen kann jedes Element im Satz unabdingbar sein, häufig sind gerade Angaben, zum Beispiel Negationspartikeln, die wichtigsten Elemente im Satz. Und andererseits können Elemente, ohne die der Satz grammatisch unkorrekt wird, unter Umständen doch eliminiert werden, solange nur die Kommunikation dadurch nicht gefährdet wird. Wir sprechen von $n \circ t$ w e $n-$ d $i \mathrm{~g}$ e $n$ bzw. we g 1 a $\beta$ b a $r$ e $n$ Elementen nur noch in diesem kommunikativen Sinne. Elemente, die auf Grund grammatischer Regeln unabdingbar sind, nennen wir o b $11 \mathrm{~g}$ a $t$ or $\mathrm{i} \mathrm{s} \mathrm{ch}$; ihre Elimination ergibt grammatisch unkorrekte (wenngleich eventuell kommunikativ brauchbare) Sätze. Obligatorisch ist ein erheblicher Teil der Ergänzungen; $E_{O}$ ist praktisch immer obliga- 


\section{Liste der wichtigsten satzmuster}

- Es taut.

o Johanna fiebert.

02 Er entledigt sich seiner Kleider.

03 Streusalze schaden den Fahrzeugen.

04 Ich denke an dich.

05 Das Dorf liegt an der Autobahn.

06 Wir fahren nach Bamberg.

07 Dieser Mann ist ein Spion.

08 Dieser Mann ist gefährlich.

o9 Er ließ die Kinder ein Lied singen.

34 Mir graut vor dir.

034 Man riet ihm zum Nachgeben.

036 Sie halfen ihm aufs Pferd.

038 Man war ihm unfreundlich begegnet.

044 Sie sprachen mit den Nachbarn über Utas Haus.

47 Es ist ein Elend mit ihm.

48 Es steht nicht gut um unsere Pläne.

048 Man ging noch recht sanft mit ihm um.
1 Es gibt keine ideologiefreie Wissenschaft. Mich friert./Es friert mich.

01 Pinkus pfeift den Kaiserwalzer.

011 Sie lehrt ihn eine neue Sprache.

012 Man beschuldigt ihn der Unaufmerksamkeit.

013 Er sagte ihr, was er dachte.

0136 Uli bringt dem Vater die Mappe ins Büro.

014 Sie überredeten das opfer zu einer angebZichen Spazierfahrt.

15 Hier gibt es das beste Brot der Welt.

015 Sie verbrachten drei Tage im Elsaß.

016 Sie brachte ihr Gepäck an den Flughafen.

017 Man nennt ihn einen Duckmäuser.

018 Man nennt ihn zwielichtig. 
torisch, wo sie vorkommt, bei den übrigen Ergänzungen hängt es vom Verb $a b$, ob sie obligatorisch sind. Elemente, die auf Grund grammatischer Regeln nicht aktualisiert $\mathrm{zu}$ werden brauchen, deren Fehlen also die Grammatizität des Satzes nicht berührt, nennen wir $f a k$ u $l$ a $t i v$. Fakultativ sind - in Abhängigkeit vom regierenden Verb - viele Ergänzungen, außerdem alle Angaben. Will man fakultative Elemente kennzeichnen, so klammert man sie zweckmäßigerweise ein.

Für den Fremdsprachenunterricht ist das Begriffspaar "obligatorisch" : "fakultativ" das wichtigere, weil es sich auf die struktur der Zielsprache bezieht; wer eine fremde Sprache lernen will, hat sich das für diese Sprache geltende Regelsystem anzueignen. Das Begriffspaar "notwendig" : "weglaßbar" hingegen ist weitgehend universeller Natur. Es kommt im Unterricht insoweit zum Tragen, als Formen der Kommunikation getestet und geübt werden. Darum ist es nur natürlich, daß dieses Begriffspaar im muttersprachlichen Unterricht, der ja eine einzelsprachliche Kompetenz schon voraussetzt, eine wichtigere Rolle spielt. als im Fremdsprachenunterricht. Es ist aber sicher schwierig, wenn nicht unmöglich, die (kommunikative) Notwendigkeit bzw. Weglaßbarkeit von Elementen in überschaubare Regeln zu bringen.

Differenziert man nun die Satzmuster weiter, indem man die fakultativen Elemente eigens kennzeichnet, so ergeben sich die $s$ a $t z$ b a u p 1 ä n e (SBP). Die Zahl der Satzbaupläne ist natürlich erheblich größer als die der Satzmuster; sie bleibt aber immer noch überschaubar. Im Valenzlexikon sind die Satzbaupläne zu jedem Verb als Zahlenkombination angegeben, wobei die Zahlen den Indizes der $E$ entsprechen und $v$ o $r$ jede fakultative Ergänzung eine Klammer gesetzt ist.

$\begin{array}{ll}\text { essen } & \text { SBP O (1 } \\ \text { erzählen } & \text { SBP O1(3 } \\ \text { erschrecken (vor) } & \text { SBP O } 4\end{array}$


Aufgrund der Satzbaupläne lassen sich die Verben in Valenzklassen ordnen; für die Struktur eines Lexikons wäre ein solches Gliederungsprinzip äuBerst wichtig .

\subsection{Beschreibungsebene}

Die dependenzielle Beschreibung blieb fast ausschließlich der Morphosyntax vorbehalten (so auch im KVL). Nun ist es aber durchaus möglich, auch semantische Elemente, seien es Wortbedeutungen oder kleinere Bedeutungselemente, depeńdenziell zu kombinieren. Der gerade hierfür in zahlreichen Spielarten verwendete Prädikatenkalkül läßt sich sogar mit ganz geringen technischen Mitteln in einen dependenziellen Kalkül übertragen. Allerdings wird dieser zweckmäßigerweise dann nicht mehr als $\mathrm{n}$ a c h g e ord ne $\mathrm{t}$ e, i n t e r $p r$ e $t$ a $t i v$ e semantik aufzufassen sein, vielmehr hat er als eine Art $g$ e $n$ e $r$ a $t i v$ e $r$ Semantik der Morphosyntax vorherzugehen. Die Gefahr solchen
Vorgehens besteht darin, daß universelle Kategorien, die hierbei notwendig verwendet werden, nicht mehr als Beschreibungsmittel sondern als Beschreibungsziel verstanden werden.

\section{A ufbaudes Lexikons}

3.1. Verben und Wörterbucheinheiten Die Anordnung der Verben im Lexikon ist alphabetisch, wobei Umlaute wie einfache Vokale behandelt werden.

Man hat oft gefordert, statt dieser Abfolge eine Gruppierung der Verben nach inhaltlichen Kriterien vorzunehmen. Es hat sich jedoch erwiesen, daß es speziell für die Klasse der Verben bisher keine geeignete Theorie gibt, um zusammenstellung nach Sachgruppen, Situationen o.ä. zu ermöglichen, die für einen Benutzer durchschaubar wären. Eine sinnvolle Verbgruppierung kann nur geleistet werden mit Hilfe einer semantischen Theorie, die Verben mit ähn- 
lichen Inhaltskonfigurationen als Elemente eines Verbfeldes ausweisen kann. ${ }^{1}$

Generell wird hier als eine Verbeinheit angesehen, was die gleiche Graphemfolge aufweist, ohne Rücksicht auf mögliche Bedeutungsdifferenzierungen und ohne Rücksicht auf unterschiedllche Umgebung. Auch die reflexiven Formen eines Verbs erscheinen unter einem Lemma mit den nicht reflexiven Formen (unterhalten, sich unterhalten). Eine Ausnahme bilden dagegen Verben wie abhöngen, erschrecken, bewegen, wachsen, wiegen, die unterschiedliche stammformen haben. Gleiches gilt auch für Verben mit unterschiedlicher Perfektbildung, die ebenfalls unter zwei Lemmata-Einheiten aufgeführt werden (irren: ist geirrt, hat geirrt). In diesem Punkt weicht das Lexikon von der Wortliste des Zertifikats DaF ab, die in solchen Fällen nur einen Eintrag aufweist.

1 Vgl. Pape, Stellungnahme und dies., Zur Methodik.
Unterschiedliche SBP beim gleichen Verb konstituieren jeweils eigene Wörterbucheinheiten mit getrennten Zelleneinträgen. Das gleiche gilt auch fur verschiedene Verbbedeutungen, wenn sie zwar den gleichen SBP erfullen, jedoch in einer Variante $\mathrm{SE}$ haben und in einer anderen nicht oder sich durch unterschiedliche Möglichkeiten der Passivbildung unterscheiden.

\subsection{Aufbau der Lexikonartikel .}

3.2.1. Die Abfolge der einzelnen Wörterbucheinheiten innerhalb eines Verblemmas richtet sich nach speziellen Sortierkriterien. Die verschiedenen Satzbauplantypen werden nach dem Prinzip der aufsteigenden $\mathrm{Ziffernfolge} \mathrm{angeordnet}(-, 0,01$, $011 \ldots . \ldots 03 \ldots 1 \ldots 8)$. Bei gleichem Satzmuster steht ein plan mit obligatorischen Ergänzungen vor dem mit fakultativen (SBP 01 vor SBP $\mathrm{O}(1)$.

Getrennte Wörterbucheinheiten werden auch dann aufgeführt, wenn - bei gleichem SBP - 
Ergänzungen in einem Fall als abhängige Sätze auftreten können, im anderen dagegen nicht. Der Ausbauplan steht dann nach dem einfachen Satzbauplan.

Bei der Differenzierung der Verben nach unterschiedlichen Passivmöglichkeiten steht die nicht-passivfähige Form vor der passivfähigen.

Bel Verben mit verschiedenen $E_{4}$ im gleichen Satzbauplan richtet sich die Abfolge der Wörterbucheinheiten nach der alphabetischen Einordnung der Präposition. Dadurch steht $\mathrm{z}$.B. die Einheit Zernen aus vor Zernen von.

Am Schluß eines Lexikonartikels stehen die Wörterbucheinheiten mit komplexem Prädikat. Dabei stehen die Einheiten mit nicht kommutierbarem es vor den Reflexivverben und diese vor den lexikalischen Fügungen .

Innerhalb der lexikalischen Fügungen erfolgt die Sortierung in der Reihenfolge:
Komplexe ohne Präposition, Komplexe mit Präposition, reflexive Gefüge. Die präpositionslosen Bildungen werden alphabetisch nach dem ersten Wort des Gefüges sortiert. Bei den präpositionalen Komplexen wird zunächst nach der Präposition geordnet. Der enklitische Artikel bei der Präposition hat auf die sortierung keinen Einfluß, so daß z.B. in und ins nicht getrennt werden. Sind mehrere Gruppen mit der gleichen Präposition vorhanden, richtet sich die weitere Sortierung nach dem Nomen, bei nicht nominalen Gruppen nach dem auf die Präposition folgenden wort.

Die reflexiven Gefüge stehen generell am Schluß und werden intern nach den oben angegebenen Kriterien sortiert.

3.2.2. Jede Wörterbucheinheit ist so aufgebaut, daß sich in der ersten Zeile links alle Informationen zum Verbalkomplex befinden. Die vorkommenden Fälle werden im folgenden Kapitel 4. beschrieben. Die mittlere Spalte enthält den Code für den Satzbau- 
plan, der im 5. Kapitel eingehend erläutert wird. Vom Satzbauplan deutlich abgesetzt steht rechts die Information zur Passivfähigkeit, uber die das 7. Kapitel handelt. Der Rest der Zelle sowie bedarfsweise die folgenden werden durch Beispielsätze eingenommen, die im 8. Kapitel diskutiert werden. Für die Informationen zu den satzförmigen Ergänzungen (SE) sind die anschließenden Zeilen vorgesehen. Dabei wird links die SE-Klasse und der jeweilige Typ aufgeführt. Zwischen SBP-Code und Passivinformation steht die Angabe zur Form der SE. Die Beschreibung der SE-Einträge findet sich im Kapitel 6 . Rechts stehen wieder die zugehörigen Beispielsätze.

Bei Einheiten, die ein Pertinenzelement zulassen, wird ein entsprechender Eintrag ebenfalls nach links gestellt, während der Beispielsatz wiederum rechts erscheint.

In einigen Fällen wird ein Wörterbucharti- kel noch durch eine besondere Bemerkung abgeschlossen.

\section{Verbalkomplex}

\subsection{Bestandteile des Verbalkomplexes}

Zum Verbalkomplex gehören drucktechnisch gesehen alle Einträge in der linken Spalte des KVL mit Ausnahme der Präpositionen. $\mathrm{Daß}$ wir die Präpositionen $\operatorname{der} \mathrm{E}_{4}$, denn nur diese sind verbspezifisch (nicht also die Präpositionen der $\mathrm{E}_{5}$ und $\mathrm{E}_{6}{ }^{1}$ ), beim Verb auführen ist rein praktisch motiviert. Die Notierung beim Verb besagt aber nicht, daß wir diese Präpositionen zum Verbalkomplex rechnen. Obwohl die Präpositionen

1 Ausgenommen von dieser Regel sind einige präfigierte Verben mit Direktivergänzung, bei denen eine feste Korrelation zwischen Präfix und Präposition der $\mathrm{E}_{6}$ besteht, z.B. abfahren von, $a b-$ holen von, abhăngen von, ausladen aus, ausziehen aus, austreten aus, aussteigen aus, eintreten in, einladen in, einziehen in, einsteigen in etc. 
$\operatorname{der} \mathrm{E}_{4}$ in der Regel nicht kommutieren ${ }^{1}$ und nur eine nicht-referentielle Bedeutung in dem Sinn haben, daß sie den Verbanschluß der Ergänzung regeln - vergleichbar den Kasusmorphemen der nominalen Elemente der $E_{0}$ bis $E_{3}$-, haben wir sie als Teil einer Ergänzungsklasse beschrieben, die durch die Eigenschaften eben dieser Präpoșitionen definiert ist. Die andere Lösung, die Präpositionen der $\mathrm{E}_{4}$ als Teil des Verbs zu rechnen, hạtte u.a. die unangenehme Konsequenz, daß Dativ- und Akkusativergänzung, in denen die $\mathrm{E}_{4}$ aufgehen würden, neu definiert werden müßten, wạs wiederum Auswirkungen beispielsweise auf die Beschreibung des Passivs hätte. Obwohl wir die Präpositionen der $\mathrm{E}_{4}$ also nicht als Teil eines Verbalkomplexes beschreiben, notieren wir sie dennoch im KVL beim Verb, um dem didaktischen

1 Bisweilen jedoch kommutieren Präpositionen der $\mathrm{E}_{4}$, z.B. bei sterben an/vor, reden von/über, sich freuen an/über.
Zweck des Lexikons Rechnung zu tragen, da die Verben mit einer $\mathrm{E}_{4}$ sinnvollerweise nur im Zusammenhang mit deren Präpositionen gelernt werden können.

Zum Verbalkomplex rechnen wir die Verben mit Verbzusatz ${ }^{2}$, die echt reflexiven ${ }^{3}$ und fakultativ reflexiven Verben ${ }^{4}$ und mit Einschränkung die lexikalischen Fügungen ${ }^{5}$ (zu denen auch die reflexiven Fügungen $z$ u rechnen sind) ${ }^{6}$. Als Teile des Verbalkomplexes sehen wir auch die Auxiliarverben, Modalverben und Modalitätsverben an, die aber nicht im KVL aufgeführt sind, da sie keinen eigenen SBP konstituieren. 7

4.2. Präfixverben und Verben mit Verbzusatz

4.2.1. Unter Präfixverben verstehen wir Verben mit einem unbetonten, untrennbaren Mor-

\footnotetext{
2 S. 4.2 .

3 S. 4.4 .2

S. 4.4 .3 .

S. 4.3 .

S. 4.4 .5 .

S. 4.6 .
} 
phem, das reihenbildend vor das Verb tritt oder zusammen mit dem Verb als isolierte Bildung vorkommt (z.B. beantragen, entscheiden, verbieten). Dagegen sprechen wir von Verben mit Verbzusatz, wenn die erste Konstituente des Verbs betont und trennbar ist. Verbzusätze können Präpositionen (einladen, umsteigen), Adjektive (kronkmelden), Adverbien ( weitererzählen, wiedergeben), Nomina auch nominale präpositionen - (radfahren, zutagetreten) und in seltenen Fällen Verben ( gehenlassen, Ziegenbleiben) sein.

Im KVL haben wir in der linken spalte beim Verbeintrag nicht zwischen Verben mit Verbzusätzen und Verben mit Präfixen unterschieden, da diese Differenzierung durch die unterschiedlichen Akzentuierungsverhältnisse regelhaft beschrieben werden kann. Die Möglichkeit der Satzrahmenbildung bei Verben mit Verbzusätzen kann aber an den Beispielsätzen abgelesen werden, da diese in der Regel im Präsens gebildet sind ${ }^{1}$, einem

1 S. 8 .
Tempus, das die verbale Klammer von Simplex und Verbzusatz auslöst.

Bei der Anordnung im KVL sind wir so verfahren, daß wir Verben mit Verbzusatz ebenso als Lemmaeinheit aufgeführt haben wie Präfixverben, wenn sie als strukturiert erkannt werden können, ihrer Bedeutung nach aber als Lexikalisierung $\mathrm{zu}$ verstehen sind (z.B. fernsehen, stattfinden, teilnehmen) und wenn sie in der Wortliste des Zertifikats DaF als Lemmaeinheit geführt werden (radfahren). In den ubrigen Fällen werden Verben mit Verbzusatz generell unter dem simplex aufgefüht. Nicht ins KVL aufgenommen haben wir Verben mit Verbzusatz, die durch eine systematische Wortbildungsregel beschrieben werden können, wie z.B. Verbverbindungen mit voll- (vollkaufen, vollschreiben, vollzeichnen ), mit- ( mitfahren, mitgehen, mitkommen, mitmachen, mitreden), weiter- (sowohl in der Bedeutung 'Fortsetzung einer Tätigkeit': weiterarbeiten, weiterlernen, weiterlesen, weiterreden, weiter 
schlafen, weitersprechen, weiterstudieren als auch im Sinne einer 'Weitergabe': weitererzählen, weitergeben, weitersagen, weiterschicken, weitersenden ), wieder- ( wiederaufbauen, wiederkommen, wiedersehen, wiedertreffen ), fort- (im Sinne einer zeitlichen Fortdauer: fortbestehen, fortdauern, fortgelten, sich fortentwickeln ) etc.

Bei den Verben mit Verbzusatz besteht das Problem, zwischen echten komplexen Verben einerseits und Simplex mit freier Ergänzung andererseits unterscheiden $\mathrm{zu}$ müssen. Um Pseudoverbkomplexe, d.h. Verben, deren erstes Kompositionsglied als Ergänzung beschrieben werden kann, auszuschließen, haben wir verschiedene Proben ermittelt.

4.2.2. Zur Entscheidung darüber, ob z.B. ein komplexes Verb oder ein Simplex mit Direktivergänzung vorliegt, haben wir die folgenden Kriterien herangezogen:

1. Kann das fragliche Element mit Präpositionalgruppen der Klasse $\mathrm{E}_{6}$ kommutieren?
Beispiele:

$$
\begin{aligned}
& \text { Komm }\left\{\begin{array}{l}
\text { herunter } \\
\text { nach unten }
\end{array}\right\} \text { ! } \\
& \text { Der Gast geht }\left\{\begin{array}{l}
\text { hinein } \\
\text { ins Hotel (hinein) }
\end{array}\right\} \text {. } \\
& \text { Der Fuchs kommt }\left\{\begin{array}{l}
\text { heraus } \\
\text { aus dem Bau (heraus) }
\end{array}\right\} \text {. }
\end{aligned}
$$

In den Fällen, in denen die Adverbien hinein und hinaus oder heraus $\mathrm{zu}$ dem Richtungspräpositionale hinzutreten, haben wir sie als fakultative, mit der Präposition korrespondierende Teil.e der $\mathrm{E}_{6}$ behandelt. 2. Ergibt die Kommutation des Simplex mit bedeutungsähnlichen Verben akzeptable Sätze?

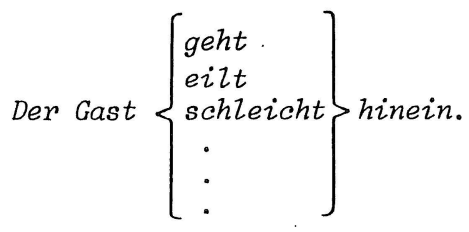

Die Probe der Anaphöisierung des fraglichen Elements fällt bei diesen Beispielen 
weg, da die Pronominaladverbien Anaphern im definierten $\operatorname{Sinn}^{1}$ sind.

Fallen die beiden Proben positiv aus, dann ist das fragliche Element - wie in den gegebenen Beispielen - eindeutig als $\mathrm{E}_{6} \mathrm{klas}-$ sifizierbar.

Damit sind als Verben mit Pseudoverbzusatz erwiesen: hinausklettern, hinübersetzen, dazwischenstellen, zurückgehen, fortlaufen, wegrennen, herunterfallen u.a.m.

Aquivalent sind die Proben, die zwischen komplexem Verb versus Simplex mit Situativergänzung entscheiden:

1. Kann das fragliche Element mit Präpositionalgruppen der Klasse $\mathrm{E}_{5}$ kommutieren?

Es stand ein alter Nußbaum $\left\{\begin{array}{l}\text { davor } \\ \text { vor dem Haus }\end{array}\right\}$. Die Katze sitzt fast immer $\left\{\begin{array}{l}\text { dahinter } \\ \text { hinter dem Kamin }\end{array}\right\}$.

1 s. 2.5 .
2. Ergibt die Kommutation des Simplex mit bedeutungsähnlichen Verben akzeptable Sätze? Die Katze $\left\{\begin{array}{l}\text { sitzt } \\ \text { hockt } \\ \text { liegt }\end{array}\right\}$ fast immer dahinter.

4.2.3. Kritische Fälle bei den Verben mit Verbzusatz sind auch die Verbindungen mit einem Adjektiv als erster Konstituente wie krankfeiern, krankmelden, sattessen, weismachen etc. Für diese Fälle sind die folgenden Proben entscheidend: ${ }^{2}$

1. Kann das adjektivische Element durch die Anaphern für $\mathrm{E}_{8}$ ( so, es, das) ersetzt bzw. durch die Fragepartikel wie erfragt werden?

\section{Beispiele:}

Macht ihre Antwort dich glücklich? - Ja, das macht sie mich.

2 S. auch Leirbukt, Vorschläge. 
Aber:

Du kannst mir nichts weismachen. - "Du kannst mir nichts so machen.

2. Kann das adjektivische Element im eingeleiteten Nebensatz bei einteiliger Konjugationsform vom finiten Verb getrennt werden?

\section{Beispiele:}

E'r hoffte, daß sie ihn glücklich dadurch macht, daß...

*Er sagte, daß du ihm weis nichts machst, was...

Bei dieser Trennbarkeit von Adjektiv und Verb ist das Adjektiv in jedem Fall als $E_{8} z u$ beschreiben. Sind Adjektiv und Verb bei dieser Probe nicht trennbar, dann ist der Aussagewert der Probe oft zweifelhaft.

4.3. Lexikalische Fügungen

4.3.1. Der Begriff der lexikalischen Fügung deckt einen Bereich ab, der noch sehr heterogen ist in bezug auf die interne syntaktische struktur wie auch in bezug auf den Verschmelzungsgrad der einzelnen Teile dieser Fügungen. Im KVL sind lexikalische Fügungen durch einen vorangestellten Stern '*' markiert. Beispiele sind: *aus den Augen verlieren, *außer acht lassen, *es weit bringen mit, *sich zum Vorbild nehmen ${ }^{1}$, *es zu tun bekommen mit u.a.m.

4.3.2. Die Besonderheit dieser Fügungen besteht darin, daß die verbabhängigen $\mathrm{Be}-$ standteile (Nomina, Adjektive, Verben, Pronomina) nicht als Ergänzungen beschrieben werden können. Entscheidend ist hier die Probe der Anaphorisierung: Sind die betreffenden Elemente nicht durch die Proformen der ihnen morphologisch vergleichbaren Ergänzungen ersetzbar, dann müssen sie als Teile eines Verbalkomplexes - wie es die lexikalische Fügung ist - behandelt werden.

Die Unmöglichkeit, die fraglichen Elemente $\mathrm{zu}$ anaphorisieren, sei an einigen Beispielen exemplarisch vorgeführt:

- Bestandteil einer lexikalischen Fügung vs. $\mathrm{E}_{1}$ :

1 Reflexive Fügungen werden in 4.4.5. behandelt. 
Sie steht dem Maler Modell.

${ }^{*}$ Sie steht es dem MaZer.

Sie trinken Brilderschaft.

* Sie trinken es/das.

- Bestandteil einer lexikalischen Fügung vS. $\mathrm{E}_{4}$ :

Ich laß mich nicht für dumm verkaufen.

* Ich laß mich nicht dafür verkaufen.

- Bestandteil einer lexikalischen Fügung vs. $\mathrm{E}_{5}$ :

Er hat die Sache im Griff.

*Er hat die Sache dort.

Seine Ausbizdung stand in Frage.

* Seine Ausbildung stand dort.

- Bestandteil einer lexikalischen Fügung vs. $\mathrm{E}_{6}$ :

Der Plan kommt zur Erörterung.

* Der Plan kommt dorthin.

Er brachte mich ganz schön in Verlegenheit.

*Er brachte mich ganz schön dorthin.
- Bestandteil einer lexikalischen Fügung vs. $\mathrm{E}_{8}$ :

Er hat es im Leben weit gebracht.

${ }^{*}$ Er hat es im Leben so gebracht.

4.3.3. Die spezielle Problematik dieser Fügungen liegt darin, daß sie zum Teil durch Attribute erweitert werden können, d.h. ihre interne struktur ist veränderbar. Die Möglichkeit einer Veränderung kann an dem Kriterium der Opposition der Artikelformen, das über weitere Attribuierungsmöglichkeiten vorentscheidet, sowie an der Numerusvariabilität festgestellt werden.

Bei den attribuierbaren Fügungen handelt es sich in erster Linie um eine bestimmte Gruppe innerhalb der sogenannten Funktionsverbgefüge, Fügungen, in denen das Veŕb als 'Funktionselement' nur noch strukturelle Verbfunktionen ausübt, während im Nomen - in der Regel einem Deverbativum der verbale Inhalt ausgedrückt ist. 1

1 S. Günther/Pape, Funktionsverbgefüge. 
Die Erweiterungen können von verschiedener Art sein. So kommutiert z.B. ein Attributsatz mit der ersten Konstituente eines zusammengesetzten Nomens bei:

Er kam in Gefahr, sein Leben zu verlieren.

Er kam in Lebensgefahr.

Andererseits kann auch ein Possessivpronomen mit einem attributiven Adjektiv, einem attributiven Genitiv und der ersten Konstituente eines Nominalkompositums kommutieren:

Das Land steht unter der Kontrolle des Militärs.

Das Land steht unter seiner Kontrolle.

Das Land steht unter militärischer Kontrolle.

Das Land steht unter Militärkontrolle.

Für uns stellte sich die Frage, wie mit den meistens von den Nomina abhängigen Elementen verfahren werden soll. Einerseits kann man sie als Attribute zum Nomen betrachten, andererseits aber auch in Abhängigkeit von der gesamten Fügung, und zwar zumindest in den Fällen, wo Ba- sisverben existieren, die mit dem Nomen der Fügung in einer Ableitungsbeziehung stehen und mit der ganzen Fügung kommutieren. Beispiele wären: in Bewegung setzen/ bewegen, in Aufregung bringen'aufregen, zur Ausführung bringen/ausführen, unter Kontrolle stehen/ kontrolliert werden, im Schlaf liegen/schlafen, in Haft nehmen/verhaften, zur Wirkung kommen/wirken, in Verdacht kommen/verdächtigt werden, in Erwägung ziehen/erwägen u.a.m. In diesen Fällen bringen die Funktionsverbgefüge oft nur eine aktionale Differenzierung gegenüber dem Basisverb zum Ausdruck. Das heißt, man könnte die Erweiterungen dieser Fügungen als "Pseudoattribute" ansehen, die den Status von Verbergänzungen haben. Das würde dazu führen, daß man neuartige Typen von Verbergänzungen ansetzen müßte, z.B. Attribute in Form von Sätzen: Gefahr Zaufen, sein Leben zu verlieren, von genitivischen Nomina: unter Kontrolle des Militärs stehen, von nominalen Präpositionsgruppen: auf eine For mulierung Wert legen und von Adjektiven: unter militärischer Kontrolle stehen. Hinzu kommt, 
daß sich die oben angeführten kommutierenden Syntagmen innerhalb der vorliegenden morphosyntaktischen Verbvalenzgrammatik nicht als Elemente eines einheitlichen $\mathrm{Pa}-$ radigmas beschreiben lassen. Als Ausweg aus diesem Dilemr. . haben wir uns dafür entschieden, keine SBP und mögliche SE zu kodieren, sondern nur den Hinweis auf transitiven oder intransitiven Gebrauch der Fügung zu geben, ohne dabei auf die Angabe von Beispielsätzen zu verzichten.

Da es sich bei den Entscheidungen über Möglichkeiten und Arten der Attribuierung weitgehend um Ermessensfragen handelt, ist der Bereich der zweifelhaften Fälle relativ groß. In dieser Lage sind wir übereingekommen, keine Untergruppierung der lexikalischen Fügungen nach Erweiterungsmöglichkeiten vorzunehmen, sondern einheitlich zu verfahren. In diesem Sinne wurde bei allen lexikalischen Fügungen - sichtbar von Simplex, Präfixverben und Verben mit Verbzusatz durch einen vorangesetzten Stern abgehoben - nur die Transitivität bzw. Intransitivität (gekennzeichnet durch $\mathrm{T}$ bzw. I) verzeichnet. Daneben sind auch die gebräuchlichsten Präpositionen dieser Fügungen bei den einzelnen wörterbucheinheiten eingetragen, z.B. Wert legen auf, Pate stehen bei, Schritt halten mit, zum Anlaß nehmen für etc. Darüberhinaus haben wir in den Beispielsätzen Attribute angegeben, und zwar nicht soweit sie überhaupt möglich sind, sondern soweit sie üblich sind, um nicht zu falschen Generalisierungen $\mathrm{zu}$ verleiten. Damit scheint uns die wesentliche Information für den Gebrauch der lexikalischen Fügungen gegeben zu sein. So gesehen dürften dem Benutzer des Wörterbuchs aus dieser anderen, aufgrund theoretischer schwierigkeiten gewählten Kodierung keine Probleme bei der syntaktischen Verwendung dieser Fügungen entstehen.

4.3.4. Metaphorische Redensarten, wie den Teufel an die Wand maien, zwei Fliegen mit einer Klappe schlagen, Eulen nach Athen tragen etc., 
die als eine Sondergruppe innerhalb der lexikalischen Fügungen angesehen werden könnten, haben wir nicht ins KVL aufgenommen. Einerseits determinieren sie die Satzstruktur weitgehend und sind daher für eine valenztheoretische Beschreibung uninteressant, andererseits sind sie im Gebrauch sehr restringiert, so daß sie ohne Angabe von Verwendungsbedingungen $\mathrm{zu}$ einem nicht korrekten Gebrauch führen können. Dagegen haben wir metaphorische Wendungen, deren Bildwert durch häufige Verwendung bereits abgeschwächt ist und die sich dadurch - wenn auch nur graduell von bildstarken Fügungen unterscheiden, im KVL aufgefuhrt, z.B. auf der Hut sein, in den Schoß fallen, auf der Hand Ziegen, sich im Kreise drehen, an den Mann bringen. Die Grenze zwischen solchen Wendungen und metaphorischen Redensarten ist allerdings fließend.

\subsection{Reflexivverben}

4.4.1. Bei der Behandlung der Reflexiva im
KVL sind zunächst folgende Gruppen zu unterscheiden:

- echt reflexive Verben

- fakultativ reflexive Verben

- partim reflexive Verben

- reflexive Fügungen

- reziproke Verben

4.4.2. Echt reflexive Verben liegen genau dann vor, wenn folgende Kriterien erfuiltt sind:

- das Reflexivpronomen ist nicht weglaßbar: $\left\{\begin{array}{c}\text { Er weigert sich, } \\ { }_{\text {Er weigert -, }}\end{array}\right\}$ das zu glauben.

- der Austausch des Reflexivpronomens gegen ein anderes Pronomen, eine Nominalgruppe o.ä. ist entweder nicht möglich: Er weigert $\left\{\begin{array}{c}\text { sich } \\ { }^{*} i h n\end{array}\right\}$ zu gehen.

oder ergibt eine Bedeutungsveränderung: Er hat $\left\{\begin{array}{l}\text { sich } \\ \text { den Motor }\end{array}\right\}$ dem Mädchen erklärt. 
Die Verben dieser Gruppe sind im KVL so erfaßt, daß das Reflexivpronomen mit Angabe seines Kasus beim Verb erscheint:

sich/Akk erkälten

4.4.3. Fakultativ reflexive Verben unterscheiden sich von den echt reflexiven dadurch, daß das Reflexivpronomen ohne Bedeutungsveränderung weglaßbar ist:

$$
\text { Er hält }\left\{\begin{array}{c}
\text { sich } \\
-
\end{array}\right\} \text { eine Illustrierte. }
$$

Der Austausch des Reflexivpronomens gegen ein anderes Pronomen, eine Nominalgruppe o.ä. ist, wie bei den echt reflexiven Verben, nicht möglich oder ergibt eine Bedeutungsveränderung.

Das Reflexivpronomen steht im KVL mit Kasusangabe beim Verb, wobei die Fakultativität - auch im Beispielsatz - durch Spitzklammern ausgedrückt ist:

〈sich/Akk > halten
4.4.4. Bei den partim reflexiven Verben ${ }^{1}$ schließlich läßt sich das Reflexivpronomen ohne Bedeutungsänderung gegen ein anderes Satzglied im selben Kasus austauschen; anders ausgedrückt: eine bestimmte E-Position kann u.a. auch durch ein Reflexivpronomen belegt werden. Aus dieser sicht rechtfertigt sich das im Lexikon eingeschlagene Verfahren, bei den Verben dieser Gruppe keine besondere Kodierung vorzunehmen; jedoch stehen bei diesen Verben je zwei Beispielsätze, von denen einer die Konstruktionsmöglichkeit mit Reflexivpronomen illustriert:

Er wäscht sich.

Er wäscht seinen Hund.

4.4.5. Unter den festen Fügungen ${ }^{2}$ sind auch solche, die ein Reflexivpronomen als festen Bestandteil enthalten. Dabei können wir zwei Typen unterscheiden:

Vgl. Stötzel, Ausdrucksseite, S. 186-192.

Vgl. dazu generell 4.3. 
- präpositional angeschlossene Reflexivpronomen + Verb

z.B. *mit sich führen

*an sich bringen

- präpositional angeschlossene Nominalgruppe (o.ä.) + obligatorisches Reflexivpronomen + Verb

z.B. *sich zur Ruhe setzen

Beide Typen sind im KVL, ebenso wie alle anderen festen Fügungen; durch einen vorgesetzten Stern gekennzeichnet und erhalten keine Angabe eines SBP.

4.4.6. Die Verben, die sogenannte reziproke Konstruktionen zulassen, bei denen das Reflexivpronomen bei gleicher Bedeutung durch einander ersetzbar ist, wie in: Sie lieben sich. wurden im Lexikon nicht erfaßt, da hier lexikalische Informationen alleine nicht hinreichen. So ist z.B. die reziproke Konstruktion nur im plural möglich.
Dies alles kann hier nur angedeutet werden, ${ }^{1}$ macht aber deutlich, warum diese Verben im Lexikon nicht gesondert behandelt werden. Hingewiesen werden muß allerdings darauf, daß nicht bei allen mit einander konstruierbaren reziproken Verben eine Ersetzung durch das Reflexivpronomen möglich ist

z.B. Sie tauschen Briefmarken $\left\{\begin{array}{l}\text { miteinander } \\ { }^{*} \text { mit sich }\end{array}\right\}$.

4.5. Nicht kommutierbares es

4.5.1. Ein weiteres Element, das im KVL beim Verb verzeichnet ist, ist das nicht kommutierbare es. Gemeint ist das es, das auftritt in sätzen wie

Es regnet.

Er nimmt es mit allen auf.

Er meint es gut mit dir.

Es gibt dort keinen Ärger mehr.

1 Eine ausführlichere Diskussion findet sich in Keim/Volkmann, Reflexiva. 
Aufgrund seiner Nicht-Kommutierbarkeit betrachten wir es nicht als Ergänzung; es erscheint also nicht im Satzbauplan.

\subsubsection{Von anderen nicht als $\mathrm{E}$ gewerteten} es- Typen, so auch vom Korrelat es 1 , unterscheldet es sich dadurch, daß es auch bel veränderter satzgliedfolge immer erscheint.

Heute regnet es.

Dort gibt es keinen Arger mehr.

Vgl. dagegen: Es friert mich. - Mich friert (es). oder: Es kamen alle meine Freunde. - Alle meine Freunde kamen.

Die in diesen Sätzen auftretenden es- Typen werden nicht im KVL verzeichnet, da sie nicht bei jeder Satzgliedstellung obligatorisch sind.

4.5.3. Von den im KVL aufgefuhrten es erscheinen manche in Rundklammern. Dies besagt, daß das betreffende es sich in bezug auf die Satzgliedfolge wie eine unbetonte $\mathrm{E}_{\mathrm{o}}$ verhät,

1 Vgl. dazu 6.3.1. und 6.3.5. d.h. es unterliegt im wesentlichen denselben Permutationsregeln wie $\mathrm{E}_{0}$. Das nicht-geklammerte es hat dagegen die ste1lungseigenschaften einer unbetonten $\mathrm{E}_{1}$.

regnen (es): Es regnet.

aufnehmen es mit-Akk: Er nimmt es mit allen auf.

4.6. El11ptische Sätze

Im KVL sind nur verben aufgefuhrt, die einen SBP konstituieren. Hilfsverben sowie Modal- und Modalitätsverben ${ }^{1}$ (Verben mit obligatorischem Infinitiv mit zu) betrachten wir als verbale Erwelterungen, die für die syntaktische Vollständigkeit von Sätzen nicht konstitutiv sind und denen auch keine valenz zukommt. ${ }^{2}$

Es gibt jedoch kritische Fälle, die elne

1 Zur Einführung des Terminus s. Neugeborn, Zur Analyse.

2 Eine andere Beschreibungsmöglichkeit von Modal- und Modalitätsverben wird in Engel, Syntax, 5.4. vorgeführt. 
Interpretation dieser Verben als Vollverben zumindest nicht ausschließen. Das ist z.B. der Fall bei sätzen, in denen als Finitum ein Hilfs- oder Modalverb auftritt und ein Infinitum aktualisierbar ist, z.B.

Er ist in die Stadt.

Ich muß leider nachhause.

Ich möchte noch germ eine Tasse T'ee.

Diese Sätze haben wir unterschiedslos als Ellipsen zu den entsprechenden Sätzen mit Infinitum aufgefaßt:

Er ist in die Stadt (gegangen/gefahren...).

Ich muß leider nachhause (gehen/fahren...).

Ich möchte noch gern eine Tasse Tee (haben).

Diese Entscheidung ist - soweit es die Sätze mit Infinitiv-Ellipse betrifft nicht problemlos. Denn es handelt sich in diesen Fällen um sehr übliche Verwendungsweisen, die nicht durch die Textkonstitution motiviert sind. Die isolierten Sätze ohne Infinitum erwecken kaum den Eindruck syntaktisch unvollständiger sätze oder den von spontaner legerer Rede (wie es bei der Ellipse des Partizips in dem Satz Er ist in die Stadt. der Fall ist).

Im Gegensatz zu den eben behandelten Fällen lassen sich für die Beschreibung von Hilfs-, Modal- und Modalitätsverben m i $t$ Infinitum als Prädikatskomplexe starke Argumente anführen, die im folgenden genannt werden sollen.

4.7. Klassifizierung verbabhängiger infinitivischer Verbalsyntagmen

Speziell bei infinitivischen Verbalsyntagmen tritt der Fall auf, daß zwischen infinitivischer SE einerseits und Infinitivkonstruktion als $\mathrm{E}_{9}$ oder Teil eines Prädikatskomplexes andererseits unterschieden werden muß. ${ }^{1}$ Dabei sind wir so verfahren, daß wir die Kommutation des fraglichen Infini-

1 S. auch Günther, Codierung und dies., Korrektur. Vgl. auch Schumacher, Probleme, S. 53-54. 
tivs mit einem nominalen Syntagma als Kriterium für selne Klassifikation als SE gewertet haben:

Er versprach seiner Frau, ihr einen Nerz zu schenken. / Er versprach seiner Frau einen Nex'z.

Er denkt daran, den Plan zu verwirklichen. / Er denkt an die Verwirklichung des Plans.

Aber:

*r traut sich nicht zu widersprechen. / "E'r traut sich keinen Widerspruch.

Ich gedenke, das morgen zu tun. / Ich gedenke des morgigen T'uns.

Es gilt zu gewinnen. / Es gilt der Gewinn.

Es beginnt, kalt zu werden. / "Es beginnt mit dem Kaltwerden.

Das Merkmal der Anaphorisierung ist bei diesen Fällen eln zu schwaches Kriterium, da es eine Klassifizierung der Infinitivkonstruktion als $\mathrm{E}$ exlauben würde, z.B.:

Es gilt zu gewinnen. / Das gizt es.

Er traut sich nicht zu widersprechen. / Das traut er sich nicht.
Mit dem Kriterium der Kommutation sind aber nur die infinitivischen SE eindeutig identiflziext. Zur Unterscheldung zwischen infinitivischex $\mathrm{E}_{9}$ und dem Infinttiv eines Modalitätsverbs mu ein weiteres $\mathrm{K} r i t e r i u m$ herangezogen werden. Dieses besteht in den unterschledlichen Rektionsverhältnissen. Bel Modalitätsverben wird das Vorhandensein einer $\mathrm{E}_{\mathrm{O}}$ und deren semantische Fỉlung von dem abhängigen Infinit1v geregelt. Dagegen wird die Aktualisierung und semantische Fullung der $\mathrm{E}_{\mathrm{o}}$ bei Verben mit einer infinitivischen $E_{9}$ vom finiten Verb und nicht vom abhängigen Infinitiv gesteuert. Die $E_{o}$ bleibt in dlesem Fall konstant; wenn man andere verben als Infinitive einsetzt:

Vollverb mit Infinitivischer $\mathrm{E}_{9}$ :

Es gilt zu gewinnen.

Es gilt, ihn willkommen zu heißen.

Modalitätsverb mit abhänglgem Infinitiv:

Er pflegt, samstags seine Einkäufe zu erledigen. In dieser Gegend pflegt es häufig zu regnen. 


\subsection{Komplexe Prädikate}

Im folgenden werden die strukturen aufgeführt, die von uns aufgrund der genannten Kriterien als Prädikatskomplexe behandelt werden:

4.8.1. Hilfsverben mit Infinitiv ohne zu bzw. mit Partizip II: haben, sein, das futurische und passivische werden und tun ( Tust du mal hereinschauen? ). Bei prädikaten mit dem Partizip II ist $z u$ prüfen, ob es sich nicht um eine $\mathrm{E}_{8}$ handelt. In diesem sinne problematisch sind sätze wie

Das Grundstück ist mit einer Mauer umgeben.

An den Wänden sind Bilder angebracht. ${ }^{1}$

\subsubsection{Weitere Konstruktionen mit Parti-} zip II:

Er gibt das Spiel vertoren.

Wo steht das geschrieben?

1 Zur Problematik der Beschreibung des sein-Passivs s. 7.1 .
Der Brief lag unter den Büchern versteckt. Er bringt die Pakete angeschleppt.

Er kommt freudig ins Zimmer gesprungen.

Ferner die passivischen. Konstruktionen:

Zum Geburtstag bekommt/erhält/kriegt er von mir ein Buch geschenkt.

Dir gehören die Haare geschnitten.

Hier gibt es etwas geschenkt.

4.8.3. Modalverben mit Infinitiv ohne zu: dürfen, können, mögen, (möchten), müssen, sollen, wolzen und nicht brauchen mit Infinitiv mit und ohne $z u$.

4.8.4. Modalitätsverben mit Infinitiv mit zu, z.B.:

scheinen zu: Er scheint zu schlafen.

pflegen zu: Sonntags pflegen sie, in die Kirche zu gehen.

sich trauen zu: Er traut sich nicht zu widersprechen.

versprechen zu: Der Urlaub verspricht, schön zu werden.

haben zu: Du hast von ihm nichts zu befürchten. 
aufhören zu: Es hört gerade auf zu regnen.

anfangen zu: Es fängt on, kalt zu werden.

Die besondere Problematik dieser Verben liegt darin; daß zu den meisten Modalitätsverben homonyme Vollverben existieren. Diese lassen sich jedoch in der Regel eindeutig von den gleichlautenden Modalitätsverben unterscheiden. Zum einen besteht zwischen homonymem Vollverb und der $\mathrm{E}_{\mathrm{O}}$ eine Verträglichkeitsrelation, während Modalitätsverben mit beliebigen $\mathrm{E}_{\mathrm{o}}$ kombinierbar sind. Bei diesen muß dagegen die Verträglichkeit zwischen $\mathrm{E}_{\mathrm{O}}$ und Infinitiv beachtet werden:

Er verspricht ihr, sie zu besuchen.

*Das Wetter verspricht uns, schön zu werden. .

\section{Aber:}

Er hat bei ihm nichts zu Zachen.

Das Buch hat hier liegenzubleiben.

Weitere Unterscheidungsmerkmale zwischen Modalitätsverben und Vollverb sind die
Anaphorisierbarkeit der abhängigen Infinitivkonstruktion beim Vollverb. Bei den Modalitätsverben kann der abhängige Infinitiv nicht anaphorisiert und auch $n$ : sht kommutiert werden:

Das Wetter verspricht, schön zu werden.

* Das Wetter verspricht es/das.

Dagegen:

Er verspricht, morgen zu kommen.

Er verspricht es/das.

und :

Das verspricht ja heiter zu werden.

*Das verspricht Heiterkeit.

Aber:

Er versprach, mir etwas zu schenken.

Er versprach mir ein Geschenk.

In einigen Fällen besteht zwischen Modalitätsverb und Vollverb auch eine unterschiedliche syntaktische Umgebung. So kann bei dem Vollverb versprechen noch eine fakultative 
$E_{3}$ stehen:

Ich verspreche (dir), morgen zu kommen.

Aber:

"Das Wetter verspricht (uns), schön zu werden.

Schwieriger ist die Unterscheidung von Modalitätsverb und homonymem vollverb bei anfangen, beginnen, aufhören, da in diesen Fällen auch bei den Vollverben die $E_{0}$ des Finitums und des Infinitivs referenzidentisch sind. Dennoch läßt sich.eine Unterscheidung durchführen, da nur die Modalitätsverben mit relativ beliebigen $\mathrm{E}_{\mathrm{O}}$ kombiniert werden können:

Die Sache fängt an, mir Spaß zu machen.

Es fängt an zu regnen.

Der Strauß fängt schon an zu welken.

\section{Aber nur:}

Klaus fängt (damit) an zu singen.

Außerdem kann nur der Infinitiv beim Vollverb anaphorisiert und kommutiert werden:
Klaus fängt mit dem Singen/damit an.

Aber:

* Die Sache fängt damit an.

*Es jängt damit an.

*Der Strauß fängt schon damit an.

Der semantische Unterschied zwischen Modalitäts- und Vollverb besteht - wie sich aus den Beispielen ablesen läßt - darin, daß das Vollverb auf eine intentionale Handlung referiert, während das Modalitätsverb lediglich den Beginn eines Prozesses bezeichnet.

Als Prädikatskomplexe betrachten wir außerdem:

4.8.5. transitive und intransitive Bewegungsverben mit Infinitiv ohne \&u:

Ich gehe noch heute in die Stadt einkaufen.

Gehst du mit baden?

Sie schickt den Jungen Brötchen holen.

Stets püntlich legt sie die Kinder schlafen. 
Das Kriterium der Kommutation hat sich bei diesen Konstruktionen als schwer handhabbar erwiesen. Einerseits schließen slch Infinitiv und $E_{6}$ im glelchen satz nicht aus:

Er geht baden/ins Freibad.

Er geht ins Freibad baden.

andererseits kommutiert der Infinitiv bei Aktualisierung einer $\mathrm{E}_{6}$ mit einem finalen Angabesatz:

Er geht in die Stadt Lebensmittel einkaufen. Er geht in die Stadt, um Lebensmittel einzukaufen.

Da im Falle der Kommutation des Infinitivs mit elner $\mathrm{E}_{6}$ die Kodierung des Infinitivs als SE theoretisch nicht vertretbar ist, haben wir uns dafür entschieden, den Inf1nitiv als Teil eines komplexen prädikats $\mathrm{zu}$ beschreiben.

4.8.6. Situativverben (sein, bleiben, haben) mit Infinitiv ohne zu:

Ich wirde heute am liebsten im Bett liegen bleiben.
Sie hat unheimlich viel Krimskrams in ihrem Zimmer herumliegen.

Schmidts haben schöne Bilder an den Wänden hängen.

Der Tisch soll so stehen bleiben.

4.8.7. Eine Gruppe von Verben, die sich ähnlich wie die Modalitätsverben verhalten:

sein: Das Problem ist kaum zu lösen.

bleiben: Es bleibt noch viel für dich au tun.

bekommen: In diesem Restaurant bekommt man noch nach 22 Uhr etwas zu essen.

kriegen: Du kriegst jetzt nichts mehr zu trinken!

geben: Gib mir doch bitte noch etwas zu trinken!

geben (es): In Koln gibt es viele schöne Kirchen zu besichtigen.

Diese Gruppe ist sehr heterogen; man kann jedoch einige Abweichungen gegenüber den Modalitätsverben feststellen:

So konkurrieren z.B. einige Fügungen mit einem modalen grammatikalisierten werdenPassiv: 
Das Problem ist kaum zu lösen/kann kaum gelöst werden.

Es bleibt noch viel zu tun/muß noch viel getan werden.

Eine weitere Abweichung besteht darin, daB bei den hier aufgeführten Verben die $\mathrm{E}_{\mathrm{O}}$ entweder nicht vorhanden (z.B. geben (es) ) oder stärker restrigiert ist (z.B. bekommen zu).

Ebenso bestehen Unterschiede in der Beschreibungsmöglichkeit des abhängigen Infinitivs bzw. der Infinitivkonstruktion. Denn bisweilen können diese als Attribute $\mathrm{zu}$ einer $\mathrm{E}_{1}$ angesehen werden, da sie mit attributiven Relativsätzen oder mit attributiven Präpositionalgruppen mit der Präposition zum und dem sustantivierten Infinitiv kommutieren:

In Köln gibt es viele Kirchen zu besichtigen. In Köln gibt es viele Kirchen, die man besichtigen kann.

und :

Auf der Geburtstagsfeier gab es eine Menge zu essen/zum Essen.
Der Grund dafür, daß wir neben dem homonymen Vollverb geben (es) mit dem SBP 15 (In Rom gibt es viele Kirchen. ) ein nicht-SBP-konstituierendes Verb geben (es) angesetzt haben, besteht darin, daß letzteres in der Konstruktion mit Infinitiv mit zu durch ein modales grammatikalisiertes Passiv (bzw. durch eine aktivische man- Konstruktion) paraphrasiert werden kann, z.B.:

In Köln gibt es viele Kirchen zu besichtigen. In Köln können viele Kirchen besichtigt werden. In Köln kann man viele Kirchen besichtigen.

Diese Paraphrasierbarkeit zeigt, daß geben (es) + Inf. mit zu als Einheit, d.h. als komplexes Verb, beschreibbar ist.

\section{Ergänzungsklassen}

5.0. Im Kapitel 2.5. wurden die einzelnen Verbalergänzungen der deutschen Gegenwartssprache definiert und kurz beschrieben. Hier soll vor allem auf Besonderheiten bei einzelnen Ergänzungen eingegangen werden. 
Vorweg sei noch einmal zwelerlei festgehalten:

1. Als Ergänzung kann immer nur gelten, was auf einem Paradigma beruht. Wo also keine Austauschbarke1t verschiedener Formen besteht, liegt auch keine Ergänzung vox. Grenzformen sind zu diskutieren.

2. Ergänzung 1st jedes Element, das (unmittelbar) von einex verbalen subklasse abhängt.

5.1. $\mathbf{E}_{\mathrm{O}}$

5.1.1. Die "Nominativergänzung" wird in grammatische Darstellungen in der Regel als "Subjekt" bezeichnet: Es ist leicht nachzuweisen, daß die von der traditionellen und den meisten Richtungen der neueren Grammatik vorausgesetzte Sonderstellung des subjekts lediglich auf die morphologische Kongruenz mit dem finiten Verb gegründet werden kann. Mlle Versuche, das subjekt semantisch $z$ u definieren als "Agens", "Handlungstrăger" u.a., sind leicht durch Beisplele widerlegbar: in den Sätzen

Der Buchhalter erhielt eine Auszeichnung.

Ich sitze gut in diesem Stuhl.

paßt keine der möglichen semantischen Definitionen. Und eine (ebenfalls semantische) Definition von der Textstruktur hex, die das subjekt als Redeansatz, als "Thema", als Topic u.ä. auffaßt, fuhrt ebenfalls in unlösbare Probleme, well alle genannten Charakterisierungen eben auch auf andere Satzglieder zutreffen können: hier hat man Merkmale, die als Funktionen anderer Strukturen anzusehen sind und gewöhn$11 \mathrm{ch}$ eng mit der Wortstellung zusammenhängen, unbedacht in den Bereich der Konnexionsstruktur ibertragen. Daß das subjekt i $n \quad v i$ e 1 e $n$ F ä 1 l e $n$ Agens ist, daß es $i \mathrm{n}$ v 1 e 1 e $n$ F ä 1 e $n$ als erstes Element des Hauptsatzes thematische Funktion hat, bleibt zweifellos von Interes- 
se: es kann aber nicht Grundlage linguistischer Definition sein.

Die augenfällige morphologische Kongruenz ist zweifellos ein wichtiges Phänomen; sie hat aber zurückzutreten hinter anderen Merkmalen wie Verbdependenz und Subklassenspezifik, die das subjekt mit anderen objekten/Ergänzungen gemein hat. Es ist deshalb sinnvoll, dieses Satzglied in Anlehnung an TESNIERE als eine unter anderen Ergänzungen anzusehen und ihm mit der Bezeichnung $\mathrm{E}_{\mathrm{O}}$ den Rang eines primus inter pares zuzuweisen.

5.1.2. Da nur von einer Ergänzung gesprochen werden darf, wo ein Paradigma vorliegt, ist das nicht kommutierbare es in Sätzen wie

Es regnet.

Es friert.

keine Ergänzung. ${ }^{2}$ Es muß als Teil des Verbs (mit SBP -) betrachtet werden. Allerdings kön-

1 Vgl. Tesnière, Eléments, S. 108-109.

2 S. 4.5 . nen Verwendungen wie in

Feuer regnet vom Himmel.

nicht übersehen werden; hier würden wir (wie vielfach bei "metaphorischem" Gebrauch) eine eigene wörterbucheinheit regnen mit eigenem SBP ansetzen. Dieses Verfahren ist nicht restlos befriedigend. "Metaphori,sche" Verwendungsweisen bereiten häufig Schwierigkeiten. Die Unterscheidung von "nichtmetaphorischem" ("eigentlichem") und "metaphorischem" Gebrauch, so problematisch sie immer sein mag, kann die Beschreibung um einiges vereinfachen.

Daß expletives und suppletives es, wie es in den Sätzen

Es ritten drei Reiter zum Tore hinaus.

und

Es ist ein Wunder, daß ihr noch nichts gemerkt habt.

vorliegt, unter den genannten Voraussetzungen keine Ergänzungen sind (sondern sekun- 
där eingefuhrte Elemente), sollte nun ke1nes Bewetses mehr bedurfen. ${ }^{1}$

5.1.3. Die $\mathrm{E}_{\mathrm{o}}$ kann, je nach regierendem Verb, in verschiedenex Ausdrucksform auftreten; so

als Nominalphrase:

$D$ e r B r i e ftr äger konnt vor $10 \mathrm{Uhr}$.

als pronomen:

E r kommt.

als Nebensatz: ${ }^{2}$

$D i c h$ a $u$ s e $h e n$, freut mich.

$D$ a $B$ d $u$ k $\mathrm{mms}$, freut mich.

u.a.

Zwar ist die $\mathrm{E}_{\mathrm{O}}$ nicht die einzige Ergänzung, die als nominativische Nominalgruppe auftreten kann. Auch die $\mathrm{E}_{7}$ - wie in

Egon ist $u$ ns e $r$ Nachbar.

1 Zum Korrelat es s. 6.3.1. und 6.3.5.

2 S. 6.1.4. kommt oft in dieser Form vor. ${ }^{3}$ Aber die Anaphortsierung zelgt, daB beide nicht verwechselt werden können. $\mathrm{E}_{7}$ erlaubt als Anapher nur es oder so, $\mathrm{E}_{\mathrm{o}}$ ein nach Genus und Numerus abwandelbares Personalpronomen Im Nominativ.

5.2. $\mathrm{E}_{1}$

5.2.1. Die "Akkusativergänzung", das traditionelle Akkusativobjekt, ist nach der $E_{0}$ die mit Abstand häufigste Ergänzung. Die melsten Verben mit $E_{1}$ haben die volle Passivfahtgkeit (z.B. sagen, bringen); Verben ohne $E_{1}$ erlauben allenfalls ein unpersönliches Passiv (z.B. helfen, bleiben ). ${ }^{4}$ Es gibt jedoch auch Akkusativverben, etwa solche mit dem "Akkusativ der Erstreckung", die nicht passivfähig sind.

5.2.2. Es muß vermerkt werden, daß in Sätzen wie

3 Vgl. 5.7.2.

4 Zum Passiv vgl. 7. 
Peter ist fünf Zentimeter gewachsen.

Die Frau hat drei Pfund abgenommen.

Der Sportzer läuft eine gute Zeit.

ein Element vorliegt, das als "Akkusativ der Erstreckung" besondere Aufmerksamkeit verdient. Sicher handelt es sich um eine Ergänzung, weil das Element nur bei einer verbalen Subklasse vorkommt. Ein Paradigma liegt in jedem Fall vor. Aber der Akkusativ der Erstreckung läßt sich nicht wie andere $E_{1}$ anaphorisieren, und er muß auch anders erfragt werden (wie lange? wie weit? (um) wieviel?). Hier liegen offensichtlich noch ungelöste Probleme. Vorderhand wird der Akkusativ der Erstreckung im Valenzlexikon unter $\mathrm{E}_{1}$ aufgeführt.

Ebenfalls zu den $E_{1}$ rechnen wir die "inneren Objekte", wie sie in einen tiefen Schlaf (schlafen), einen schönen Traum (träumen), einen schweren Gang (gehen) u.a. vorkommen. Es liegt zwar in der Regel nur ein minimales Paradigma vor, jedoch sind die Gefüge mit Einschrän- kungen passivfähig und anaphorisierbar. Das Nomen ist auch in jedem Fall attribuierbar. ${ }^{1}$

Bei den Verben der sinnlichen Wahrnehmung (z.B. hören, sehen) kann außer der Nominalphrase im Akkusativ auch ein Infinitivsatz ohne zu angeschlossen werden, der seinerseits eine nominale oder pronominale Phrase im Akkusativ enthält:

Wir sahen die kinder noch weglaufen.

Wir sahen ihn kommen.

Auf Grund der Anaphorisierbarkeit durch das werảen diese Nebensätze als $E_{1}$ klassifiziert. 2 5.2.3. Gelegentlich treten $P$ e $r$ i $n$ e $n z-$ e 1 e $m$ e $n t$ e im Akkusativ auf, so bei treten (neben Pertinenzdativ ${ }^{3}$ ):

Er trat mich auf die Zehen.

Er trat mir auf die Zehen.

1 Vgl. 4.3.

2 Vgl. 6.4.3.

3 S. 5.4 .4 . 
Der Pertinenzakkusativ wird im Valenzlexikon aufgefuhrt, allerdings nicht als $\mathrm{E}_{1}$. Nicht kommutierbares es wie in dem Satz

Paul versucht es mit einem Brief. ist keine $\mathrm{E}_{1}$ sondern Teil des Verbs. 1 Von der $E_{1}$ unterschieden werden muß auch die $E_{7}$ als Nominalgruppe tm Akkusativ. Sie tritt immer dann auf, wenn das betreffende Verb außer der $\mathrm{E}_{7}$ eine $\mathrm{E}_{1}$ fordert:

Er nannte ihn einen Dunnkopf.

Durch die Anaphorisierung mit so sind dabet jedoch Verwechslungen ausgeschlossen.

5.2.4. Die $\mathrm{E}_{l}$ weist im Gegensatz zu den anderen Ergänzungsklassen die Besonderheit auf, daß sie bei einigen Verben doppelt auftritt, ${ }^{2}$ allerdings mit unterschiedlichen Restriktionen

\section{S. 4.5 .}

2 Für den etwas anders gelagerten Fall der doppe1ten $\mathrm{E}_{4} \mathrm{vgl}$. 5.5 .
Er lehrte sie die französische sprache.

Bei diesen Fällen enthält die erste $E_{1}$ ein Nomen mit dem Merkmal "persönlich", während bei der zweiten $E_{1}$ dem Nomen das Merkmal "unpersönlich" zukommt.

Die Besonderheiten bei den Verben des Gebens und Nehmens werden unten behande1t. ${ }^{3}$

5.2.5. Mögliche Ausdrucksformen von $E_{1}$ sind

Nominalphrase:

Ich habe $m e i n e n$ B $r$ u $e r$ gesehen.

Pronominalphrase:

Ich habe $i h n$ gesehen.

Nebensatz:

Ich sehe $i h n k$ om $m e n$.

Ich sehe, $d$ a $B$ e r k 0 m $t$.

Ich sehe, $\quad b$ e r k 0 m $t$.

Ich sehe, woher e r kommt.

Ich sehe, e r kom $\mathrm{m}$.

3 S. 5.5 .5 . 


\section{3. $\mathrm{E}_{2}$}

Es ist oft betont worden, daß das "Genitivobjekt" ein seltenes und allmählich aussterbendes objekt sei. Daran ist soviel richtig, daß die $E_{2}$ nur (noch) bei einigen verben (z.B. bedürfen, sich enthalten) vorkommt und daß sie in einigen Fällen in Konkurrenz mit $\mathrm{E}_{4}$ steht, so bei sich besinnen.

Ich besinne mich auf meine Pflichten.

Ich besinne mich meiner Pflichten.

Hier überwiegt heute die $E_{4}$, und es ist anzunehmen, daß die $E_{2}$, die in diesen Fällen ohnehin auf gehobene Sprache beschränkt ist, in absehbarer Zeit aus dem Sprachgebrauch verschwinden wird. ${ }^{1}$ Auf der anderen Seite ist die $E_{2}$, wo sie nicht in Konkurrenz mit einer anderen $\mathrm{E}$ steht, als durchaus "stabil" anzusehen. Dies gilt vor allem für verben aus dem juristischen Bereich, so für bedürfen, be-

1 Ublich ist die $\mathrm{E}_{2}$ hier nur in Verwendungen wie sich eines Besseren besinnen. schuldigen, bezichtigen und andere; die meisten dieser Verben sind nicht im Valenzlexikon enthalten.

Die $\mathrm{E}_{2}$ kommt vor allem als Nominal-bzw. Pronominalgruppe vor, in Satzform allenfalls als generalisierender Nebensatz:

Wessen er angeklagt wurde, kann er sich nicht mehr entsinnen.

5.4. $\mathrm{E}_{3}$

5.4.1. Das "Dativobjekt" der traditionellen Grammatik kommt bei Verben wie helfen, nützen, trauen u.a. vor. Probleme weist hier vor allem die Abgrenzung von anderen dativischen Phrasen auf, die nicht als $E_{3}$ gelten können. ${ }^{2}$ Relativ einfach ist die Unterscheidung der $E_{3}$ vom $D$ a $t i v$ u e $t h i c u s$, wie er in dem Satz Du bist $m i r$ ein schöner Freund! vorliegt. Der Dativus ethicus kommt nur in

2 Zu den Nominalgruppen im Dativ vgl. Engelen, Untersuchungen, S. 116-123. 
emotiv markierten sätzen vor, er ist nur als Personalpronomen realisierbar, nicht betonbar und nicht erststellenfähig. Da die $E_{3}$ auch in nicht emotiv markierten sätzen vorkommt, als Nominal-wie als Pronominalgruppe realisierbar, auBerdem betonbar und erststellenfähig ist, bestehen keine weiteren Abgrenzungsprobleme.

5.4.2. Schwieriger ist die Unterscheidung von $E_{3}$ und $D$ a $t i v u s ~ s y m ~ a t h i-$ c u s (oder Dativus commodi), wie,er in dem Satz

Ich öffne $d i r$ die Tür.

vorliegt. Denn dieser Dativ ist auch als Nominalgruppe (Ich öffne $m e i n e m \quad V$ a $e^{r}$ die Tur. ) realisierbar, er ist betonbar und erststellenfähig ( $M$ e $i n$ e $V$ a t e $r$ habe ich die Tür geöffnet. ). Was ihn von $\mathrm{E}_{3}$ unterscheidet, ist die generelle Ersetzbarkeit durch eine für-Phrase:

Ich öffne für meinen Vater die Tür.
Da dies bei $\mathrm{E}_{3}$ unmöglich ist - man kann nicht sagen: "Ich helfe fïr meinen Vater. ist die Abgrenzung gegenüber dem Dativus sympathicus gesichert.

5.4.3. Als eine Variante des Dativus sympathicus kann der $D$ a $t i v$ u $i n-$ c o m m o d i gelten; beide sind gewissermaßen Antonyme. Der Dativus incommodi liegt vor in dem Satz

Das Glas ist $m i r$ auf den Boden gefalzen.

Dieser Dativ ist betonbar und erststellenfähigi er ist außerdem als Nominal-wie als Pronominalgruppe realisierbar. Da er aber im allgemeinen einen negativen, unerwünschten oder unerwarteten Gesamtinhalt des satzes voraussetzt, kann er im Gegensatz zum Dativus commodi nie durch das inhaltlich positive für-Gefüge ersetzt werden. Im einzelnen gibt es sehr verschiedenartige Ersatzmöglichkeiten.

Was den Dativus incommodi (ebenso wie ethicus und sympathicus) von der $\mathrm{E}_{3}$ unterschei- 
det, ist die Tatsache, daß sie i $n$ j e d e m $F$ a 11 e $w$ e $g 1$ a $\beta$ b a $r$ sind. Die $\mathrm{E}_{3}$ hingegen ist, je nach regierendem Verb, obligatorisch oder fakultativ.

5.4.4. Zusätzliche Probleme weist der $P$ e $r$ i $n$ e $n z d$ a $t$ i auf, wie er in dem Satz

Er klopfte dem Bruder auf den Für?ken. vorliegt. ${ }^{1}$ Es gibt übrigens noch weitere Pertinenzelemente, so den Pertinenzakkusativ. ${ }^{2}$

Alle Pertinenzelemente bezeichnen Menschen oder höhere Lebewesen, denen Gegenstände, Körperteile oder auch Eigenschaften auf besonders enge Weise zugeordnet werden, die als $E_{0}, E_{1}, E_{5}$ oder $E_{6}$ realisiert sind.

Der Pertinenzdativ kommt demnach nur bei Verben vor, die eine $E_{0}, E_{1}, E_{5}, E_{6}$ regieren; insofern ist auch er subklassenspezi-

1 Vgl. v. Polenz, Pertinenzdativ.

2 S. 5.2 .3 fisch. Daß es sich hier aber nicht um eine Verbergänzung handelt, zeigt sich darin, daß sein Vorkommen von der lexematischen Realisierung eben dieser genannten Ergänzungen abhängt. In dem Satz

Er schlug auf den Tisch.

ist kein Pertinenzdativ möglich, weil Tisch nicht im beschriebenen sinne einem höheren Lebewesen zugeordnet ist ( ${ }^{*} E r$ schlug ihm auf den Tisch. wäre ungrammatisch.). Daraus folgt, daß der Pertinenzdativ wie alle anderen Pertinenzelemente nur mittelbar vom Verb, unmittelbar jedoch von dem Nomen einer Verbergänzung abhängt.

Trotzdem erscheint uns der Pertinenzdativ als so wichtiges Phänomen, daß er im Valenzlexikon aufgefürt wird.

5.4.5. Dativus ethicus, sympathicus und incommodi hingegen, obwohl sie strenggenommen als Verbergänzungen gelten müssen, werden im valenzlexikon nicht notiert, und 
zwar vor allem deshalb, well die Zahl der Verben, die mit einem dieser Dative verbunden werden können, fast unübersehbar wäre.

Die $\mathrm{E}_{3}$ kommt vor allem als Nominal- oder Pronominalphrase vor, daneben als generaiisierender Nebensatz: ${ }^{1}$

Karl hilft seinem Großvater.

Karl hilft ihm.

Karl hilft, wem er kann.

5.5. $\mathrm{E}_{4}$

5.5.0. In der traditionellen Grammatik liegt, sofern Elemente wie die $\mathrm{E}_{4}$ überhaupt behandelt werden, häufig ein relativ unklarer Begriff des "Präpositionalobjekts" vor. Entweder sind nämlich alle präpositionalen Satzglieder gemeint, und dann handelt es sich.nicht um eine reine Satzgliedkategorie, weil auch präpositionale Attribute inbegriffen sind;

1 S. 6.2.2. oder es 1st nur ein Teil der präpositionalen Elemente gemeint, jedoch ohne daß die erforderliche Definition gegeben würde. Diese begriffliche Unschärfe besteht weithin auch noch in modernen Grammatiken. 5.5.1. Aus der Zahl der Ergänzungen, die als Präpositionalphrasen realisierbar sind, läßt sich die $E_{4}$ durch zwel Kriterien abgrenzen:

1. Die Präposition ist nicht austauschbar. Dies gilt z.B. in folgenden Sätzen

Hans wartet auf Anna.

Wir denken an den Urlaub.

Austauschbarkeit liegt hingegen vor in den ebenfalls häufig präpositionalen $E_{5}$ und $E_{6}$ :

Er wohnt in Bamberg/am Meer/neben Heinz.

Ich fahre nach Bamberg/ans Meer/ins Gebirge.

2. Die Präposition ist immer in der $E_{4}$ enthalten:

Wir denken an den Urlaub/an ihn/daran. 
In $E_{5}$ und $E_{6}$ kann die Präposition

auch fehlen (falls die Ergänzung

durch ein "Adverb" wiedergegeben ist):

Er wohnt dort.

Ich fahre hin.

Auf diese Art ist die Unterscheidung von $E_{4}$ einerseits, $E_{5}$ und $E_{6}$ andererseits im allgemeinen problemlos. Folge der Nichtaustauschbarkeit der Präposition ist, daß dieser in der Regel keine eigene Bedeutung zukommt; jedenfalls bedürfte es mancher Verrenkungen, um dem auf beim Verb warten (auf) elne eigene, vom Verb losgelöste Bedeutung zuzuschreiben. Es gibt allerdings Grenzfälle, in denen die Präposition bedeutungsdifferenzierend wirkt, so bei

sich freuen an

sich freuen auf

Bei der weiteren Variante

sich freuen über ist kaum $\mathrm{zu}$ entscheiden, ob ein $\mathrm{Be}-$ deutungsunterschied $\mathrm{zu}$

sich freuen an

vorliegt oder nicht. Es ist aber unmöglich, diese Bedeutungsunterschiede allein den Präpositionen zuzuordnen. Im KVL haben wir drei verschiedene wörterbucheinheiten, alle mit der Valenz $O(4$, jedoch mit je eigener Präposition angesetzt.

Problematisch sind ferner Fälle wie

schreiben für

schreiben gegen

(beide Verwendungsweisen von schreiben sind nicht ins KVL aufgenommen worden). Bei schreiben in der Bedeutung 'schriftlich Partei ergreifen' haben die Präpositionen unzweifelhaft eigene, scharf unterschiedene Bedeutungen. Aber das Paradigma ist auf zwei Antonyme beschränkt, es fehlt die relativ freie 
Austauschbarke1t der Präpositionen, wie sie fur Adverblalbestimmungen typisch 1st. Genaue Zuordnungskr1terien mussen also fur solche Verben noch festgelegt werden.

5.5.2. Bel Verben mit $\mathrm{E}_{4}$ wird Im KVL Immer die jeweilige Prăpositton hlnter dem Verbeintrag angegeben. Zusătzlich erfolgt eln Elntrag des Kasus der nachfolgenden. Nominalgruppe, der durch die Präposition gesteuert wird, in folgender Form:

hoffen auf - Akk

AuBer Nominalgruppen Im Akkusativ und Dativ können. In einıgen Fällen auch Adjektive nach einer Präposition auftreten. Diese Fä1le werden durch den Eintrag

$$
\text { halten für - Adj }
$$

markiert. In der Regel kann alternativ zum Adjektiv auch elne Nominalgruppe stehen:

Ich halte es $\left\{\begin{array}{l}\text { für falsch, } \\ \text { für einen Fehler, }\end{array}\right\}$ daß...
Ein Sonderfall liegt bel bis vor, das hier unter die präpositionen gerechnet wird. Da auf bis außer Nominalgruppen auch andere Prăpositionen wie zu und in folgen können, die threrseits den Kasus der nachfolgenden Nominalgruppe bestimmen, wird bet Verben, bei denen die $E_{4}$ mit bis. anschließt, auf eine weitergehende Kennzeichnung verzlchtet.

Die sitaung dauert $\left\{\begin{array}{l}\text { bis } 10 \mathrm{Uhr} . \\ \text { bis zum späten Abend. } \\ \text { bis in den Morgen. }\end{array}\right.$

5.5.3. Da die Präposition in. der $\mathrm{E}_{4}$ fest mit dem Verb verbunden 1st, hat man gelegentlich vorgeschlagen, sle uberhaupt dem Verb zuzurechnen. Dafür spricht die Bedeutungsleere der Präposition in der $\mathrm{E}_{4}$, die der Bedeutungsleere des Kasus "an sich" gleicht. Und der Fremdsprachenunterricht hat daraus die Konsequenz gezogen: man lernt sich exinnem an, sich beschäftigen mit usw., genau so wie man lernt essen mit. Akkusativ, helfen mit Dativ usw. Folge eines solchen Verfahrens wäre efnfach, daß den Kategorien 
$E_{1}$ und $E_{3}$. erheblich mehr Elemente zuwuichsen' und die Kategorie $\mathrm{E}_{4}$ wegfiele.

Dre1 Gründe sprechen jedoch dagegen, die Präpositionen der $\mathrm{E}_{4}$ dem regierenden verb zuzurechnen: 1

1. Die Präposition bildet mit den von ihr abhängigen Elementen, ưbrigens nicht nur im Falle der Pronominaladverbien, deren Bestandteil sie ist, einen besonders engen Komplex (die Präpositionalphrase), die auf die genannte Weise auseinandergerissen würde.

2. Die Tatsache, daß gerade viele $\mathrm{E}_{4}$ fakultativ sind, macht eine Integration der Präposition in das regierende Verb unzweckmäßig. Denn wir können zwar Ergänzungen als fakultativ kennzeichnen, nicht aber Teile von Verben. Die Folge wäre, daß man bei jedem verb mit. fakultativer $\mathrm{E}_{4}$ zwei wörterbucheinhei-

1 Vgl. 4.1. ten ansetzen müßte, z.B.

sich exinnern

und

sich erinnern an.

Das Lexikon würde dadurch ganz erheblich aufgebläht.

3. Außerdem gibt es eine Anzahl von Verben, die zwei $\mathrm{E}_{4}$ mit verschiedenen Präpositionen regieren, deren Nominalgruppen in teilweise unterschiedlichen Kasus auftreten:

Er spricht mit ihm über etwas.

Oft ist es so, daB mindestens eine $\mathrm{E}_{4}$ aktualisiert sein muß, aber auch beide gemeinsam auftreten können. Wir verwenden in diesen Fällen bei SBP die Codierung 04v4. Es wäre auch in diesen Fällen sehr schwierig, die Präpositionen in den Verbalkomplex $\mathrm{zu}$ integrieren. 


\subsubsection{Phrasen mit der Prăposition bei} als regierendem Element sind in der Regel Adverbialbestimmungen mit austauschbarer Präposition. Im Sonderfall einiger kommunikativer Verben wie sich melden bei wird aber das präpositionale Element als nicht kommutierend angesehen und als (hier fakultative) $\mathrm{E}_{4}$ kodiert. Zwar sind auch hier andere Präpositonen möglich (sich in der Zentrale melden), aber diese schließen das meist personale bei-Element nicht aus:

Er meldet sich in der Zentrale beim Operateur.

Hier würde neben der $\mathrm{E}_{4}$ noch eine Lokalangabe angesetzt.

5.5.4. Phrasen mit regierendem mit sind meist entweder Instrumentalbestimmungen -

Ich mache das mit dem Harmer.

- oder Komitativbestimmungen (sie geben die begleitenden Umstände an) :

Inge geht mit ihrem Freund ins Kino.

Walter geht mit dem Schirm in die stadt.
Die Komitativbestimmungen werden hier als Angaben angesehen, da sie nicht verbsubklassenspezifisch sind. AuBerdem kommutiert das komitative mit mit in Begleitung (von), in Gesellschaft (von) bzw. unter Mitnahme (von), nicht ohne $\mathrm{u} . \mathrm{a}$. Solche Phrasen werden deshalb im KVL nicht eigens angegeben.

Bei den Instrumentalbestimmungen liegt der Fall komplizierter. Zwar kommutiert auch hier die Präposition mit mittels, mit Hilfel Unterstutzung (von), unter Verwendung (von) u.a. Jedoch sind Instrumentalphrasen spezifisch für die Subklasse derjenigen Verben, die Tätigkeiten oder Handlungen bezeichnen.

In den meisten Fällen gibt es bei diesen Verben auch die Möglichkeit, das Instrument mit einer $\mathrm{E}_{\mathrm{O}} \mathrm{zu}$ bezeichnen, so in

Der Stein traf das Fenster.

Die semantische Beziehung dieses Satzes zu Hans traf das Fenster mit dem Stein.

ist, neben der Subklassenspezifik, ein zu- 
sätzliches Argument fưr die Behandlung der Instrumentalphrasen als fakultative $\mathrm{E}_{4}{ }{ }^{1}$

Daneben gibt es auch andere mit- Phrasen, die zweifellos $z u$ den Ergänzungen gehören, so bei streiten (mit). Sie werden somit als $\mathrm{E}_{4}$ codiert. Bei reziprokem Gebrauch der Verben erscheint die $\mathrm{E}_{4}$ als miteinander: ${ }^{2}$

Inge und Walter streiten miteinander.

Das Element miteinander ist jedoch häufig fakultativ, besonders bei reziprokem Gebrauch reflexiver Verben:

Hans und Oskar unterhalten sich miteinander. Hans und Oskar unterhalten sich.

5.5.5. Verben des Gebens und Nehmens wie essen, geben, nehmen, trinken, versuchen haben in der Regel eine $E_{1}$, die das Gegebene/ Genommene bezeichnet:

Er bringt mir Kartoffeln.

Rolf versucht die Schokolade.

1 Vgl. Ballweg, Hans trifft das Fenster.

2 Vgl. 4.4.6.
Anstelle dieser $E_{1}$ ist meist auch eine $E_{4}$ mit von möglich, wenn deutlich gemacht werden soll, daß es sich um Tellmengen handelt: Er bringt mir von den Kartoffeln.

Rolf versucht von der Schokolade.

Diese Alternative gilt so generell, daß sie, von Ausnahmen abgesehen, nicht eigens kodiert wird: Wo jedoch akkusativisches und präpositionales Element zusammen vorkommen - wie in

Rolf versucht ein Stück von der Schokolade.

- betrachten wir das präpositionale Element nicht als selbständiges Satzglied, sondern als Attribut zur $E_{1}$.

5.5.6. Es ist, auch in neueren Grammatiken, häufig davon die Rede, daB die $\mathrm{E}_{4}$ in vielen Fällen eine ursprünglich andere Ergänzung, namentlich eine $E_{2}$, "verdrängt" habe. Als Beispiel seien aufgeführt

sich einer sache erinnerm

sich an etwas erinnern 
Solche Entwicklungen sind jedoch fưr ein wörterbuch, das nur den gegenwärtigen Sprachgebrauch aufweisen will, ganz unwichtig. Deshalb wurde im KVL nur die Form

$$
\text { sich erinnern (an) } O(4
$$

kodiert und der heute ganz unübliche Genitiv nicht eigens vermerkt.

5.5.7. Gegenủber anderen Ergänzungen, besonders $E_{2}$ und $E_{3}$, weist die $E_{4}$ eine Vlelzahl von Ausdrucksformen auf.

Es kommen vor

Präpositionalphrase mit Nomen:

Walter erinnert sich an einen Freund.

Präpositionalphrase mit pronomen oder Determinativ:

Walter erinnert sich an ihn/diesen.

In diesen Fällen wird der Kasus der abhängigen Phrase von der Präposition bestimmt, das KVL enthält einen entsprechen- den Vermerk.

"Pronominaladverb":

Walter erinnert sich doran.

Diese Form alterniert mit Präpositionalphrasen mit Pronomen/Determinativ. Letztere werden gewöhnlich für personen und (höhere) Tiere gebraucht, erstere für Sachverhalte. Bei unbelebten Gegenstånden sind bei de Formen möglich:

Walter erinnert sich an den alten Tisch. Walter erinnert sich daran. Walter erinnert sich an ihn.

Nebensatz mit Korrelat: ${ }^{1}$

Rolf denkt daran zu kommen.

Rolf denkt daran, daß er hingehen sollte.

Rolf denkt daran, wohin er gehen sollte.

Nebensätze ohne Korrelat:

Walter erinnert sich, daß er hingehen wollte. usw.

1 Vgl. 6.3.2. und 6.3.5. 
neben

Walter erinnert sich daran, daß er hingehen wollte.

5.6. $E_{5}$ und $E_{6}$

5.6.1. In der traditionellen Grammatik treten diese beiden Ergänzungen - wenn überhaupt - unter Bezeichnungen wie Umstandsergänzung, Umstandsbestimmung, Adverbialbestimmung, Richtungsbestimmung u.a. auf. Selten werden sie kategoriell voneinander getrennt, und fast nie werden sie von $\operatorname{der} \mathrm{E}_{4}$ unterschieden. Den letztgenannten Mangel weisen allerdings auch die meisten. modernen Grammatiken auf.

Der Augenschein mag dafür sprechen, $E_{5}$ und $\mathrm{E}_{6}$ in einer Ergänzungsklasse zusammenzufassen, denn beide werden als einzige Ergänzungen durch Partikeln realisiert, die man gewöhnlich Adverbien nennt. Aber die Unterschiede überwiegen. Vor allem ist die Menge der Verben mit $\mathrm{E}_{5}$ von der Menge der Verben mit $\mathrm{E}_{6}$ deutlich verschieden; nur weni- ge Verben kommen wahlweise mit $\mathrm{E}_{5}$ oder $\mathrm{E}_{6}$ vor. ${ }^{1}$ Außerdem sind die jeweiligen Anaphern deutlich verschieden. Beide Ergänzungen werden hier nur deshalb zusammen behandelt, weil so die Notwendigkeit ihrer Trennung leichter nachgewiesen werden kann.

5.6.2. $\mathrm{E}_{5}$ liegt vor in Sätzen wie

Helmut wohnte in Wasseralfingen.

Die sitzung beginnt um $10 \mathrm{Uhr}$.

Anaphern sind da bei lokaler und dann bei temporaler Präpositionalgruppe. Meist handelt es sich bei der $E_{5}$ um eine Lokalergänzung. Nur bei wenigen Verben wie starten (Das Flugzeug startet um $8 \mathrm{Uhr}$. Das Flugzeug startet in Frankfurt.) kommen temporale neben lokalen Bestimmungen vor oder nur temporale wie bei angehen (Das Theater geht um $20 \mathrm{Uhr}$ an.).

Lokale wie temporale $\mathrm{E}_{5}$ benennen unzweifelhaft die Situation, in die ein Sachverhalt eingebettet ist. Deshalb wird die $E_{5}$ hier

$1 \mathrm{Zu}$ den Ausnahmen vgl. Pape, Bemerkungen, s. 42. 
auch $s$ i $t$ a $t 1$ v e r g ä $n z u n g$ genannt.

Die Ausdrucksformen der $E_{5}$ sind nicht sehr zahlreich. Hauptsächlich erscheint die $\mathrm{E}_{5}$ in zwei Formen:

als "adverblale" partikel/Partikelphrase:

Helnut wohnt dort.

Helmut wohnt genau dort.

Er lebt weit droben.

als Präpositionalphrase mit austauschbarer Präposition:

Helmut wohnt in der Stadt.

Helmut wohnt am FZuß.

Satzförmige Ergänzungen ${ }^{1}$ kommen nicht vor.

Generallsierende Nebensätze:

Horst wohnt, wo gestern der Waldbrand war.

Da bei der $\mathrm{E}_{5}$ wie auch bei der $\mathrm{E}_{6}$ mehrere Präpositionen in Betracht kommen, haben

1 Vgl. 6.2. wir im KVL auf eine Aufzählung verzichtet.

5.6.3. Im Bere1ch der Situativbestimmungen 1st zwischen Ergänzungen und Angaben

nicht. immer leicht $\mathrm{zu}$ unterschelden. Obligator1sche Satzglieder können (da Angaben immer fakultativ sind) nur Ergänzungen sein. Daher ist die ortsbestimmung bel wohnen eine Ergänzung, we11 *Er wohnt. eln ungramutischer Satz wäre. Auch bei leben (in Sätzen wie Sie lebt in Feudenheim. ) liegt eine $\mathrm{E}_{5}$ vor. Zwar hat der.Satz Er Zebt. als grammatisch zu gelten. Da es sich aber um verschiedene Bedeutungen von leben handelt - im ersten Fall 'slch aufhalten, wohnen', im zwelten Fall 'am Leben seln' -, kann man zwe1 wörterbucheinheiten mit verschiedener valenz ansetzen. Schwierigkeiten bereiten Verben wie starter, wo die Lokalbestimung fakultativ ist. Hier ließen wir uns von der Erwägung leiten, inwleweit die Erwartung elner Lokalbestimmung begründet ist. Bei besonders hohem Erwartungsgrad wurde die Lokalbestimmung dann als Ergänzung klassifiziert. Es muß betont 
werden, daß sich in solchen Fällen keinesfalls "beweisen" läßt, ob Angabe oder Ergänzung vorliegt, darum können auch Zählungen unsere Klassifikation nicht zwingend beeinflussen. In gewissen Grenzfällen bleibt es dem Grammatiker nicht erspart, nach $\mathrm{Ab}-$ wägung aller Gesichtspunkte Entscheidungen $z u$ fällen und $z u$ vertreten. Jedenfalls helfen Proben wie die unter 2.4 erwähnten (z.B.: Bestimmte Satzglieder sind Angaben, wenn sie durch einen Nebensatz vertreten werden können) auch nicht viel weiter, wenn sie nur zusätzliche Kriterien für Begriffe liefern, die vorher anderweitig definiert worden waren.

sichtbar etwas mit ihnen $z u$ tun haben.

5.6.4. Vieles von dem zur $E_{5}$ Gesagten gilt auch für die $E_{6}$. Sie liegt vor in den Sätzen

Helmut fährt nach Berlin.

Erwin kommt aus Heidelberg.

Anapher ist hin oder dorther/daher. Beide
Partikeln haben im Neuhochdeutschen nur lokale, jedenfalls keine temporale Bedeutung; $E_{6}$ sind also immer Ergänzungen lokalen Inhalts. Sie geben in jedem Fall, indem sie Ziel, Ausgangspunkt oder den passierten Bereich nennen, die Richtung eines Vorgangs an. Deshalb wird die $\mathrm{E}_{6}$ auch $D i r e k t i v e r g a ̈ n z u g$ genannt.

Ausdrucksformen sind, wie bei $\mathrm{E}_{5}$, Partikeln/Partikelphrasen -

Er fährt hin.

Er zielt genau dorthin.

- oder Präpositionalphrasen mit austauschbarer Präposition:

Regine kam aus Göttingen.

Regine fuhr nach stuttgart.

Satzförmige Ergänzungen kommen nicht vor, wohl aber generalisierende Nebensätze: ${ }^{1}$

1 Vgl. 6.2.2. 
Wohin du gehst, will ich auch gehen.

Im Bereich der Direktivbestimmungen gibt es nicht nur Ergänzungen. Wieder gilt, daß obligatorische Elemente allemal als $E_{6} \mathrm{zu}$ gelten haben, von den fakultativen die, denen wir elnen besonders hohen Erwartungsgrad und damit eine spezifische Relation zum Verb zuschreiben. Wird diese Bedingung nicht erfüllt, so liegt eine Direktivangabe vor. Würde diese Einschränkung nicht gemacht, so wirrden zahllose Verben die Valenz 6 , aufweisen, auch wenn sie nur selten und nur unter besonderen Bedingungen mit einer Richtungsbestimmung zusammen vorkommen: man vergleiche

Er sang aus dem Fenster.

Er keuchte den Gang entlang.

Er Zächelte hinüber.

usw.

In all diesen Sätzen, die nur ausnahmsweise mit Richtungsbestimmungen vorkommen, sprechen wir von Angaben; nur die verhältnismäßig kleine Gruppe der eigentlichen "Richtungsverben" wie fahren, gehen, Zaufen, reisen u.a. kommen mit $\mathrm{E}_{6}$ vor.

5.6.5. Es ist gelegentlich kritisiert worden, daß $E_{5}$ und $E_{6}$ "semantisch definiert" seien und daß, da zum Beispiel $E_{0}$ bis $E_{4}$ morphologisch definiert sind, die Kriterien für die Klassiflkation der Ergänzungen disparat seien. 1 Diesem Vorwurf kann folgendes entgegengehalten werden: Die Ergänzungen beruhen auf Kommutationsreihen, die, da nicht Beliebiges mit Beliebigem kommutieren kann (sondern nur sich irgendwie entsprechende Elemente in jeweils sinnvollen Sätzen), ohnehin erhebliche semantische Voraussetzungen machen; insofern war ja auch der taxonomische strukturalismus keineswegs so asemantisch, wie sich viele seiner Vertreter gerierten. Uber diese allgemeine semantische Voraussetzung hinaus wurde bei der Ermittlung von $\mathrm{E}_{5}$ und

1 Vgl. z.B. Schumacher, Probleme, S. 52. 
und $E_{6}$ nicht mehr semantik ins spiel gebracht als bei den zuvor aufgeführten Ergänzungen: auch dort enthielt die Kommutationsreihe (das Paradigma) jeweils eine Anapher, mit deren Hilfe eine eindeutige Identifikation der Ergänzung möglich wurde. Im übrigen kann es angesichts der Doppelnatur sprachlicher Zeichen (mit Ausdrucksund Inhaltsseite) nicht wundernehmen, wenn sich zu oberflächensyntaktisch ermittelten Kategorien stellenweise (sekundär) semantische Entsprechungen ergeben (wie bei $\mathrm{E}_{5}$ und $E_{6}$ ): angesichts des Umstands, daß es keine grundsätzliche 1:1-Relation zwischen Ausdruck und Inhalt gibt, darf allerdings das Fehlen solcher Entsprechungen (zum Beispiel bei $E_{O}$ bis $E_{4}$ ) auch kein Wehgeschrei auslösen.

\section{7. $\mathrm{E}_{7}$ und $\mathrm{E}_{8}$}

5.7.1. In der traditionellen Grammatik kommen diese beiden Elemente teilweise gar nicht vor, weil sie einfach als Teile des "Prädi- kats" begriffen werden, in dem dann das Verb (meist sein, werden, bleiben) nur noch die unselbständige Rolle einer "Kopula" spielt. Als Prädikatsteile werden sie jedoch von vielen Grammatikern (substantivisches bzw. adjekt1visches) Prädikatsnomen genannt.

GLINZ und die inhaltbezogene schule wollten der "Kopula" wieder den Rang eines vollwertigen Verbs zugestehen und kamen - auf Grund einer Verkennung der durch das Verb ausgedrückten Beziehung - zu Bezeichnungen wie "GleichgröBe" (GLINZ), "Gleichsetzungsnominativ" (GREBE) für die $\mathrm{E}_{7}$ " "Artangabe" (GLINZ), "Artergänzung" (GREBE) für die $\mathrm{E}_{8} \mathrm{bzw}$. einen Teilbereich von ihr. ${ }^{1}$ Eine zumindest für die $\mathrm{E}_{7}$ notwendige neue Bezeichnung wird unten begründet.

Es bestehen ausreichende Gründe, die bei-

1 Vgl. die Synopse s. $22 / 23$. 
den Ergänzungen zusammen $z u$ behandeln. Sie weisen in der Tat viele Gemeinsamkeiten auf. Vor allem handelt es sich - und das unterscheldet sie von dem Paar $E_{5} / E_{6}-$ fast immer um dieselben Verben, die mit $\mathrm{E}_{7}$ und $\mathrm{E}_{8}$ vorkommen, vor allem um sein, werden, bleiben, nennen. Außerdem gelten größtentells dieselben Anaphern. Es gibt Forscher, die einen Klassenunterschied zwischen beiden nicht anerkennen oder höchstens eine subklassifikation zulassen wollen. Eine solche Lösung muß ernsthaft diskutiert werden. Im KVL werden zwei getrennte Klassen angesetzt vor allem, well die Kernlexeme je verschieden sind (Nomen bzw. Adjektiv).

5.7.2. $\mathrm{E}_{7}$ kommt meist als Nominalphrase im Nominativ oder (bei Vorliegen einer $E_{1}$ ) im Akkusativ vor:

Manfred ist ein Musiker.

Man nennt Manfred einen sanften Menschen. Mein Schwager heiBt Manfred.

Sie hießen ihn den Großen Vorsitzenden.
In bestimmten Fällen wird $E_{7}$ durch Phrasen mit wie oder als wiedergegeben:

Er galt als ein zuverlässiger Mann.

Er heißt wie sein Vater.

Man bezeichnete ihn als einen zuverlässigen Mann.

Man nannte ihn wie seinen Vater.

Wären als und wie Präpositionen, so müßten die Ergånzungen als $\mathrm{E}_{4}$ klassifiziert werden. Präpositionen sind jedoch hier als wörter definiert, die, falls sie eine Nominal- oder eine Pronominalgruppe regieren, einen spezifischen Kasus verlangen. Genau dies trifft bei als und wie nicht zu: der Kasus der ihnen folgenden Phrasen richtet sich nach dem eines anderen Satzglieds (der $\mathrm{E}_{\mathrm{O}}$ oder der $\mathrm{E}_{1}$ ). Aus diesem Grund können von als oder wie eingeleitete Phrasen keine $\mathrm{E}_{4}$ sein.

Anapher ist in den erstgenannten Fällen so oder (unveränderliches) es, bei den als-Phrasen ein-: 


\section{Manfred ist es.}

Man nennt ihn so.

Mein Schwager heiBt so.

Sie heiBen ihn so.

Er galt als einer.

Man bezeichnete ihn als einen.

$\mathrm{Da}$ es unveränderlich ist, kann - selbst bei Gleichklang mit pronominalem es aus $E_{0}$ oder $E_{1}-E_{7}$ nicht mit $E_{0}$ oder $E_{1}$ verwechselt werden. Wichtig für die Unterscheidung von der $E_{8}$ ist, $d a \beta$, sofern keine Anapher vorliegt, der Nukleus der $E_{7}$ immer ein Nomen ist.

5.7.3. An Ausdrucksformen von $\mathrm{E}_{7}$ kommen neben den schon genannten Nominalphrasen und Anaphern nur generalisierende Nebensätze vor:

Er heißt, wie es ihm paßt.

Er gilt, als was er will.

Der primär morphosyntaktisch definierten $\mathrm{E}_{7}$ kommt - ähnliches gilt übrigens auch für $E_{8}$ - ein ziemlich eindeutiges seman- tisches Merkmal zu. Es läßt sich allerdings mit Bezeichnungen wie "Gleichsetzung" nicht fassen - die aufgeführten Beispiele zeigen mit hinreichender Deutlichkeit, daß hier nichts "gleichgesetzt" wird. Sie weisen eher auf eine ElementMenge-Beziehung hin. Wenn man aber den Satz

Viele Touristen in Italien sind Deutsche. betrachtet, so zelgt sich, daß die zuvor gebrachten Beispiele nur Grenzfälle darstellen. Prinzipiell geht es um die Relation von Untermenge $z u$ Obermenge, und die - in Schulbüchern, überhaupt in Lehrbeispielen so beliebte - Einermenge ist nur eine, wenngleich häufige, Form der Untermenge. Sätze mit $E_{7}$ geben also immer eine Mengeninklusion wieder: eine Untermenge wird einer Obermenge subsumiert oder eingeordnet. Deshalb nennen wir die $E_{7}$ auch $E i n \circ r d n$ u $g$ s e $r g a ̈ n z$ u $n g{ }^{1}$.

1 Bei Engel, Syntax: Subsumptivergänzung. 
5.7.4. Kategorielle Nähe zur $\mathrm{E}_{8}$ ist freilich unubersehbar. Dies zeigen schon die folgenden Beispiele:

Brigitte ist verträumt.

Man nennt Brigitte verträumt.

Sie gilt als liebenswert.

Man bezeichnet sie als liebenswert.

Auch die Anaphern gleichen sich: so oder unveränderliches es, bet als-Phrasen am ehesten solch-:

Brigitte ist soles.

Man nennt sie so.

Sie gilt als solche.

Man bezeichnet sie als solche.

Die $\mathrm{E}_{8}$ enthält, wo keine Anapher vorliegt, immer ein Adjektiv als zentrales Lexem.

An Ausdrucksformen kommen neben den in den Beispielen angegebenen Formen wiederum generalisierende Nebensätze vor: ${ }^{1}$

1 Vgl. 6.2.2.
Man nennt sie, wie man will.

sie gilt, als was sie will.

Auch die $\mathrm{E}_{8}$ ist (sekundär) semantisch charakterisierbar. In jedem Falle geht es um eine Art von Qualifikation. Wir nennen die $E_{8}$ deshalb A $r$ e $r g a ̈ n z u n g{ }^{2}$. 5.7.5. Die Unterscheidung von $\mathrm{E}_{7}$ oder $\mathrm{E}_{8}$ stößt bel der Anaphorisierung auf Schwierigkeiten. Es und so kommen bei $\mathrm{E}_{7}$ wie auch bei $\mathrm{E}_{8}$ vor. Hinzu kommt, daß die alsPhrasen bei $E_{7}$ auch eine Anaphorisierung durch solch- erlauben wïrden (well die meisten $\mathrm{E}_{7}$ a $\mathrm{u} c \mathrm{~h}$ qualifizierende Funktion haben) und daß die Anaphorisierung der als-Phrasen bei $E_{8}$ überhaupt nicht völlig überzeugend durchführbar ist. Denkbar wäre durchaus elne Lösung, die alle alsPhrasen der Kategorie $\mathrm{E}_{8}$ zuweist (mit solchals Anapher). Wir sind allerdings hier anders verfahren.

2 Bei Engel, Syntax: Qualifikativergänzung. 
Die Abgrenzung von $\mathrm{E}_{7}$ und $\mathrm{E}_{8}$ gründet sich ausschließlich auf die zentralen Lexeme (Nomen bei $E_{7}$, Adjektiv bei $E_{8}$ ). Es ist klar, daß diese Kriterien bei den Anaphern (und bei den generalisierenden Nebensätzen) versagen. Aber hier zeigt sich zugleich eine wichtige Einschränkung der Operationalisierbarkeit des von uns angewandten Klassifikationsverfahrens: Anaphern lassen sich syntaktisch nur dann einwandfrei charakterisieren, wenn klar ist, wo f ü $r$ sie stehen, $w$ o $r$ a f sie verweisen. Man muß also, um eine Anapher der entsprechenden Klasse zuordnen $\mathrm{zu}$ können, das gesamte $\mathrm{Pa}-$ radigma oder doch seine wesentlichen Teile kennen. Bei den meisten Ergänzungen ist das völlig unproblematisch. Schwierigkeiten können sich aber ergeben, wenn so oder unveränderliches es zu klassifizieren sind. Das Dilemma, das übrigens nur bei der Textanalyse und nie bei der sprachproduktion auftritt, läßt sich nicht völlig befriedigend lösen. In den meisten Fällen wird zwar der Kontext klären, weil eine Anapher selten ohne vorausgehendes konkreteres Bezugselement vorkommt. In den übrigen Fållen hat der Textinterpret die zuordnung vorzunehmen; helfen können dabei die Begriffe subsumption und Qualifikation, wie sie oben umrissen wurden. Es erübrigt sich zu sagen, daß Gleiches für die Klassifikation generalisierender Nebensätze aus $\mathrm{E}_{7}$ bzw. $E_{8}$ gilt.

5.8. $\mathrm{E}_{9}$

5.8.1. In dem komplexen Satz

Mein Bruder läßt volztanken.

bildet das infinitivische Verb volztanken unzweifelhaft eine Ergänzung, weil es nur als von einer Subklasse von Verben abhängiges Element realisierbar ist. Die meisten Grammatiken gehen auf diese Ergänzungsklasse überhaupt nicht ein. Stellenweise findet man Bezeichnungen wie "abhängiger Infinitiv" oder "Infinitivkonstruktion", ohne 
daß damit eine nähere Charakterisierung als Satzglied verbunden wäre. Nur KAUFMANN spricht vom "Infinitiv als Ergänzung"."

5.8.2. Ergänzungen in Form von Nebensätzen sind uns freilich schon oben begegnet. G e ne r a 1 is i e re nde $N$ e b e n $s \ddot{a} t z$ e sind bei allen bisher besprochenen Ergänzungen ( $E_{0}$ bis $E_{8}$ ) möglich. Bei $\mathrm{E}_{\mathrm{O}}, \mathrm{E}_{1}$ und $\mathrm{E}_{4}$ kommutieren außerdem sonstige Nebensätze mit verschiedener Ausdrucksform mit einfachen (= nichtsatzförmigen), Elementen; wir sprechen in solchen Fällen von s a $t z$ f ö $r$ m $g$ e $n$ E r g ä $n z u n-$ $g$ e $n^{2}$.

Die $\mathrm{E}_{9}$ unterscheidet sich von generalisierenden Nebensätzen und von satzförmigen Ergänzungen lediglich dadurch, daß sie

$n i c h t m i t e i n a c h e n E l e-$ $m$ e $n t e n$ k $m$ m $t i$ e $t .{ }^{3}$ Es han-

Vgl. Kaufmann, Grammatik, s. 30-32.

2 Bei Engel, Syntax: Ausbausätze.

3 Vgl. 4.6. und 6.1. delt sich also um obilgatorische Nebensätze in Ergänzungsfunktion. Wir nennen die $E_{g}$ auch $E$ r gä $n z$ u $g$ s $s$ a $z .^{4}$ 5.8.3. $E_{9}$ kommen nur bei einer kleinen Anzahl von Verben vor. Sie haben übrigens - gesteuert von den regierenden Verben verschiedene Ausdrucksformen:

daß-Satz,

z.B. bei "unpersönlich" gebrauchtem heißen:

Es heißt, daß dicke Menschen gemutlich sind.

Heißen hat hier die Valenz 9; das es ist unveränderlich, also kein Satzglied.

Infinitivsatz ohne $z u$, vor allem bei Zassen ('veranlassen' oder 'zulassen') :

Mein Bruder läßt den Wagen waschen.

Infinitivsatz mit $z u$,

z.B. das "unpersönlich" gebrauchte gelten:

4 Bei Enge1, Syntax: Verbativergänzung. 
Es gilt, den Preis zu gewinnen.

5.8.4. Bei den infinitivischen Ergänzungssätzen ist darauf $z u$ achten, daB alle Ergänzungen außer einer $E_{0}$ nicht vom Hauptverb des Satzes ( Zäßt/heiBt/gilt), sondern vom abhängigen Infinitiv ( waschen/sein/gewinnen) abhängen. Ein strukturdiagramm für das letzte Beispiel sieht also folgendermaßen aus:

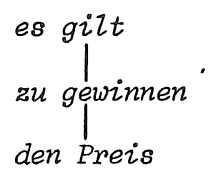

mit Klassensymbolen:

oder detaillierter:

$$
\left.\right|_{E_{9}} ^{V}
$$

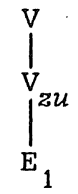

Deutlicher wird dies noch bei komplexeren Sätzen wie

Vater läßt die Kinder das Zimmer aufräumen.

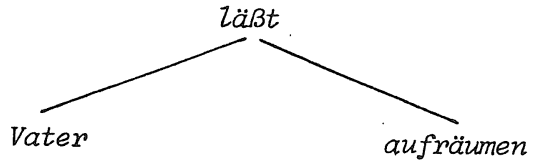

die Kinder

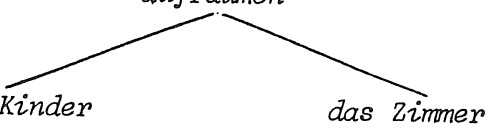

Verwendet man auch hier Symbole, so ergibt sich

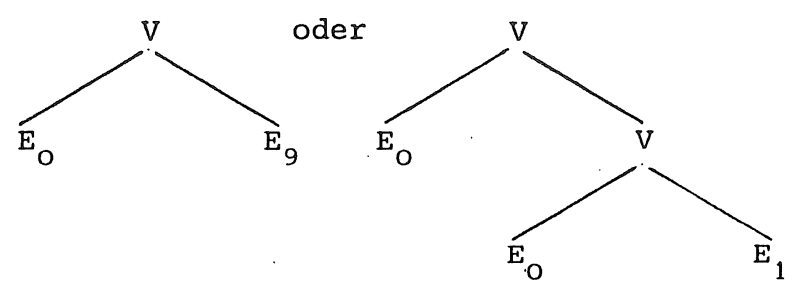

die Kinder ist zweifellos als $\mathrm{E}_{\mathrm{O}}$ des $\mathrm{ab}-$ hängigen Verbs aufräumen $\mathrm{zu}$ betrachten. Es erscheint aber hier als Akkusativ. Offenbar liegt eine Regel zugrunde, nach der innerhalb jeder $\mathrm{E}_{9}$ die entsprechende $E_{o}$ in ein Akkusativelement transformiert wird. Wir schreiben deshalb korrekter: 


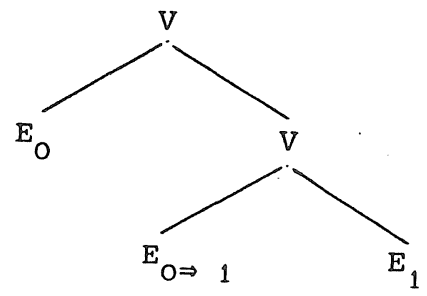

5.8.5. Die Anaphorisierung der $\mathrm{E}_{9}$ bereitet keine nennenswerten Schwierigkeiten. Da jede $E_{9}$ (als Nebensatz) ein Verbalsatz ist, kann nur mittels eines Verbs anaphorisiert werden. Verben mit besonders allgemeiner Bedeutung sind geschehen und tun. Wir wählen deshalb als Anapher der $\mathrm{E}_{9}$ (es) geschehen bzw. (es) (zu) tun bei Infinitivsätzen, daß/als ob es geschieht/ geschähe bzw. als ob jemand es tut/täte bei den übrigen Nebensätzen. Das führt zu anaphorisierten Sätzen wie

Er Zäßt es geschehen.
Es gizt, es zu tun.

Es heißt, daß es geschieht.

\section{Satzförmige Ergänzungen}

6.1. Definition der SE

6.1.1. Prinzipiell ist es möglich, daß alle Satzglieder in Form eines Satzes auftreten. Solche Sätze werden hier Gliedsätze genannt. Sie lassen sich in Ergänzungsund Angabesätze untergliedern. In diesem Zusammenhang sind die Angabesätze nicht von Interesse, da sie nicht valenzbedingt sind ( We $n n d$ e $r$ H $h n k r \ddot{a} h t$, ist die Welt noch in Ordnung. Oder: Wir mußten uns beeilen, we $i$ i e in Gewitter aufkam.). Bei den verbleibenden, als Verbergänzungen fungierenden Gliedsätzen lassen sich zwei Gruppen unterscheiden:

1.) Solche, die die $E_{0}$ bis $E_{8}$ unbeschränkt vertreten können und somit nichts beitragen zur weiteren Untergliederung der durch die Satzbaupläne konstituierten Verbsubklassen, 
und

2.) solche, die nur bei bestimmten Ergänzungen und nicht bei allen Elementen einer Verbsubklasse möglich sind.

6.1.2. Die ersteren - sie sollen generalisierende Ergänzungssätze heißen sind immer möglich, vorausgesetzt, die Ergänzung ist vom Satżbauplan her zugelassen. Beispiele für generalisierende Ergänzungssätze sịnd etwa:

We $r$ das $l i$ est, ist gescheit.

Oder: Ich helfe, wem ich will.

Oder: Sieweik, was er damit gemeint hat.

Da sie innerhalb der Verben eines Satzbauplans nicht distinktiv sind, können sie hier vernachlässigt werden.

6.1.3. Von Belang dagegen sind die unter 2.) genannten Gliedsätze. Sie werden hier satzförmige Ergänzungen (SE) genannt. Mit Hilfe der SE läßt sich eine Spezifizierung der Verbsubklassen erreichen, deren Elemente alle denselben Satzbauplan haben. So ist aus semantischen Gründen z.B. nicht bei allen Verben mit dem Satzbauplan 01 eine $\mathrm{SE}$, möglich ( $E r$ fragt, $o b$ s $i e$ kommen wol le . Oder: Er versichert, $d a B \cdot e r$ von alledem nichts $g e w u \beta t h a b e$. Aber: "Er kauft ein, $d a B . / \circ b \ldots$.$) . Hierbei werden die Ursa-$ chen, die in der Verbsemantik liegen, nicht analysiert; lediglich der Effekt in Form der SE wird registriert. SE sind keinesfalls $\mathrm{zu}$ verwechseln mit $\mathrm{E}_{9}{ }^{1}$. Wenn eine $\mathrm{SE}$ möglich ist, kann immer auch eine "einfache" E stehen (Er versichert, daß ... - Er versichert seine Unschuld. ). Dies ist bei $\mathrm{E}_{9}$ gerade nicht der Fall. ( Jetat heißt es, die Sache anders anzufassen. - ${ }^{*}$ Jetzt heißt es das. ) 6.1.4. Außerdem sind $\mathrm{SE}$ abzugrenzen von Nebensätzen, wie sie die folgenden Beispie-

1 Vgl. 5.8.2. 


\section{le enthalten:}

Die Frage, $o b$ s $i e k \circ m$ e $n$ wollen, bewegt ihn.

Seine Versicherung, $d a \beta$ e $r$ o $n$ a $l i e-$ dem. nichts $g e w u \beta t h a b e$, hilft uns nicht.

Ich.bin gespannt, $o b$ e $r$ mi $r$ a $r$ a $u f$ $a n t w o r t e t$.

Hier handelt es sich um Sätze, die nur mittelbar verbabhängig sind. Direkt hängen sie vom Nukleus einer $E_{0} b z w . E_{8} a b$, und da sie Attribute zu Nomen bzw. Adjektiv darstellen, werden sie Attributsätze genannt. Diese lassen sich ihrerseits wieder danach unterscheiden, ob sie subklassenspezifisch für die Klasse Nomen bzw. Adjektiv sind oder nicht. Im ersteren Fall liegen Ergänzungen vor, Im letzteren Angaben. Aber dies zu registrieren ist Sache eines Nomen- oder Adjektivvalenzlexikon. Deshalb bleiben hier die Attributsätze außer Betracht.

$\mathrm{Zu}$ erwähnen ist ein Sonderfall, bei dem die Abhängigkeitsverhältnisse komplizierter sind. In Beispielen wie

Es ist fraglich, $o b$ e r kom $\mathrm{m}$.

$D a \beta$ e $r$ k $\mathrm{mm}$, finde ich erfreulich.

vertreten die Nebensätze eine $E_{0} b z w_{1} E_{1}$ und hängen damit direkt vom Verb ab. Das Auftreten und die. Form dieser sàtze ist aber weniger durch das Verb (sein bzw. finden) gesteuert als durch das Adjektiv (fraglich bzw. erfreuzich), d.h. durch die $E_{8}$. Das zeigen die folgenden ungrammatischen Beispiele:

*Es ist verrostet, ob er kommt.

* $O b$ er kommt, finde ich erfreulich.

Trotz diesen Interrelationen zwischen $\mathrm{E}_{8}$ und einer anderen $E$ werden sie nicht als Attributsätze, sondern als SE behandelt aufgrund ihrer direkten, durch Kommutationstests $\mathrm{zu}$ veranschaulichenden Abhängigkeit vom Verb.

$$
\left\{\begin{array}{l}
\text { ob er kommt, } \\
\text { Die sache ist }
\end{array}\right\} \text { ist fraglich. }
$$


$\left\{\begin{array}{l}\text { Daß er kommt, } \\ \text { Seinen Entschluß }\end{array}\right\}$ finde ich erfreulich.

Hinzu kommt, daß dem Benutzer des KVL einige wesentliche Informationen verlorengegangen wären, hätte man sich in diesen nicht eindeutigen Fällen für eine andere Handhabung entschieden.

6:1.5. Die nunmehr gegenüber den verschiedenen anderen Nebensätzen abgegrenzten SE konstituieren die sogenannten Ausbaupläne. Es handelt sich hierbei um Satzbaupläne, bei denen eine oder mehr Ergänzungen als SE realisiert werden können. Wenn ein Ausbauplan vorliegt, ist das im KVL daran erkennbar, daß unter dem entsprechenden Verb die Ausbaumöglichkeiten mit'dazugehörigen Beispielsätzen aufgeführt sind.

6.2. SE-Klasse

6.2.1. Die SE werden wie die "einfachen" E klassifiziert. Um welche SE-Klasse es sich handelt, läßt sich an der Eintragung, die sich direkt unter dem Verb befindet, ablesen. Dort ist beispielsweise vermerkt enthält also eine $E_{1}$ in Form einer SE. Im KVL erscheinen die folgenden SE-Klassen:

die folgenden SE-Klassen:

$\mathrm{SE}_{\mathrm{O}}$ : Es beruhigt mich, dich zu sehen.

Es interessiert mich, warum du das zu mir gesagt hast.

Es ist ärgerlich, daß er imner zu spät kam.

$\mathrm{SE}_{1}:$ Ich nehme an, du hast nach der Zangen Reise Hunger.

Ich hoffe, daß du mir häufig schreibst.

Ich überlege 〈es〉 mir grïndlich, ob ich mit dir ins Kino gehe.

$\mathrm{SE}_{2}:$ Er kann sich nicht besinnen, diesen Brief erhalten zu haben.

$\mathrm{SE}_{4}$ : Wir haben uns 〈dazu〉 entschlossen, den Vorschlag anzunehmen.

Er überzeugt sich〈davon〉, daß sein neuer Anzug gut sitzt.

Ich bin mir im unklaren darüber, wie ich das Problem lösen soll.

6.2.2. Wenn $E_{3}$ und $E_{5}$ bis $E_{8}$ als Glied- 
sätze realisiert werden, liegen general1sierende Ergånzungssätze vor ${ }^{1}$ und kelne SE. Bei $E_{9}$ handelt es sich per definitionem immer um einen Ergänzungssatz. ${ }^{2}$

Obgleich die generalisierenden Ergänzungssätze im KVL nicht berücksichtigt werden, sei im folgenden für jede der E-Klassen, die keine SE haben, ein Beispiel mit einem generalisierenden Ergänzungssatz angeführt:

$\mathrm{E}_{3}:$ Er vertraut, wem er kann.

$\mathrm{E}_{5}$ : Er wohnt, wo Fuchs und Hase sich Gutenacht sagen.

$\mathrm{E}_{6}:$ Ich folge dir, wohin du gehst.

$\mathrm{E}_{7}$ : Er ist, was seine Väter schon waren.

$\mathrm{E}_{8}$ : Sie ist nicht, wie ich sie mir vorgestellt habe.

Sie behandelt ihn, als ob/wie wenn er ein Kind wäre.

Sie behandelt ihn, als wäre er ein Kind.

6.3. SE-TYP

6.3.1. Die SE werden im KVL nicht nur da-

1 Vgl. 6.1.2.

2 Vgl. 5.8.2. nach unterschieden, welche Ergänzungsklassen sie vertreten, sondern auch nach SE-TYP, je nach dem, ob sie mit obligatorischem, fakultativem oder ohne Korrelat stehen.

Die Information über den SE-TYp findet sich im KVL nach der Angabe der SE-Klasse. Unter Korrelat verstehen wir sprachliche Elemente wie es, damit, darauf, dazu .... die mit einer im selben Satz auftretenden. SE im Zusammenhang stehen. ${ }^{1}$ Es ist Korrelat zu $\mathrm{SE}_{\mathrm{O}}$ und $\mathrm{SE}_{1}$; alle anderen Elemente korrelieren mit $\mathrm{SE}_{4}$, wobei die verbspezifische Präposition bei $\mathrm{E}_{4}$ sich wiederfindet als Teil des Korrelats zu SE 4 (mit-damit, auf - darauf, zu-dazu ... ).

6.3.2. Korrelate haben zwar dieselbe Form wie Anaphern ${ }^{2}$, sie sind jedoch aufgrund ihrer Funktion anders $\mathrm{zu}$ werten. Von Ana-

1 Vgl. auch Köhler, Korrelate in Gliedsätzen, S. 200.

2 Vgl. 2.4. und 5.0 . 
pher sprechen wir bei der Bestimmung von E-Klassen, wenn es also darum geht, daß ein möglichst abstraktes Element als Ergänzung auftritt. Ein Korrelat dagegen kann $1 \mathrm{~m}$ e $r$ n u r $z$ u s ä $t z$ $11 \mathrm{c} \mathrm{h} z u$ einer (satzförmigen) Ergänzung stehen. Die Zusammenhänge zwischen Korrelat und $\mathrm{SE}$ können verschiedenartig sein. Wenn z.B. bei einem Verb eine $S E$ nur dann möglich ist, wenn im selben Satz ein Korrelat steht, sprechen wir von obligatorischem Korrelat.'Aus Gründen, auf die unten noch näher einzugehen ist, findet sich die Bemerkung "mit obligatori- . schem Korrelat" im KVL nur bei $\mathrm{SE}_{4}$. Beispiele dafür sind etwa:

Er baut fest $d$ arauf, einen Kredit zu bekommen.

Er baut fest $d$ a $r$ a $u f$, daß sein Freund verschwiegen ist.

Es geht ihm vor allem d a $r u m$, ob er den Prozeß gewinnen kann.

Das Kind litt d a r unter, daß es immer allein war.
Auch bel veränderter Satzgliedstellung muß das obligatorische Korrelat stehen: der Vollständigkeit halber sei ein Beispiel aufgeführt, obwohl der satz leicht schwerfällig wirkt, wenn die $\mathrm{SE}_{4}$ vor dem finiten Verb steht:

Damunter, daß es immer allein war, litt das Kind.

6.3.3. Bei einer großen Gruppe von Verben ist der zusammenhang zwischen Korrelat und SE weniger eng. Mag das Korrelat nun die Aufgabe haben, auf die SE hinzuweisen oder sie hervorzuheben; jedenfalls handelt es sich hier um den SE-Typ mit fakultativem Korrelat. Es ist in den Beispielsätzen mit Spitzklammern gekennzeichnet. Dafür, ob das Korrelat schließlich gesetzt wird oder nicht, können stilistische Erwägungen entscheidend sein. ${ }^{1}$ Wir haben bei $S E_{1}$ die Beobachtung gemacht,

1 Vgl. Ballweg, 1:SE mit fakultativem Korrelat. 
daß es in kurzen sätzen oft weggelassen

wird: Sie glaubt ihm, daß er sie liebt. In längeren Sätzen dagegen; die z.B. einige Angaben enthalten, ist ein Korrelat durchaus üblich: Sie glaubt es ihm nur alzzu gerne, daß er sie liebt. vom fakultativen Korrelat $z u \mathrm{SE}_{4}$ läßt sich nicht behaupten, daß es in kurzen Sätzen ungewöhnlich sei:

Ich denke darüber nach, warum du das gesagt hast. kann man genau so gut sagen wie

Ich denke nach, warum du das gesagt hast.

Bei längeren Sätzen scheint allerdings auch bei $\mathrm{SE}_{4}$ die Wahrscheinlichkeit für das Auftreten des Korrelats zu steigen:

Ich denke oftmals und immer ohne Ergebnis darüber nach; warum du das gesagt hast.

Beim SE-Typ mit fakultativem Korrelat verzeichnet also das KVL lediglich mögliche Strukturen. Bei einer didaktischen Verarbeitung ist zusätzlich der stilistische Aspekt zu berücksichtigen:
6.3.4. Der dritte SE-Typ ist der ohne Korrelat. Beispiele hierfür sind etwa:

Ich nehme an, du hast nach der langen Reise Hunger. ( $\left.\mathrm{SE}_{1}\right)$

Sie beschlossen, ihn zu entlassen. ( $\mathrm{SE}_{1}$ )

Ich meine, daß wir nachhause fahren solzten. ( $\left.\mathrm{SE}_{1}\right)$

Fritzchen hört auf zu Zernen. $\left(\mathrm{SE}_{4}\right)$

6.3.5. Bei einer Reihe von Verben findet sich im KVL an der stelle, wo sonst der SE-Typ angegeben ist, ein Verweis auf die Einleitung. Dies ist bei allen $\mathrm{SE}_{\mathrm{O}}$ und bei einigen $\mathrm{SE}_{1}$ der Fall. ${ }^{1}$ In Sätzen mit $\mathrm{SE}_{\mathrm{O}}$ ist das Korrelat es immer dann obligatorisch, wenn das Vorfeld des Satzes nicht anderweitig besetzt ist:

Es ärgert mich, daß du nicht gekommen bist. Ist das Vorfeld besetzt, so kann das Korrelat als fakultativ gelten, vorausgesetzt, die $\mathrm{SE}_{\mathrm{O}}$ steht im Nachfeld:

1 Vgl. Ballweg-Schramm, Korrelat und Satzgliedstellung. 
Mich ärgert $\langle e s\rangle, d a ß d u$ nicht gekommen bist. Jetzt ärgert 〈es〉 mich, daß du nicht gekommen bist.

Tritt schlieblich die $\mathrm{SE}_{\mathrm{O}}$ ins Vorfeld, so kann kein Korrelat stehen:

Daß du nicht gekommen bist, ärgert mich.

Bei den fraglichen $\mathrm{SE}_{1}$ - es handelt sich, genauer gesagt, um die $\mathrm{SE}_{1}$, die weder dem Typ "mit fakultativem Korrelat" noch dem Typ "ohne Korrelat" zugeordnet sind - ist das Korrelat dann obligatorisch, wenn sie im Nachfeld stehen:

Ich finde es schade, daß wir den Berg nicht be stiegen haben.

Steht die $\mathrm{SE}_{1}$ aber im Vorfeld, so ist wiederum ein Korrelat unmöglich:

Daß wir den Berg nicht bestiegen haben, finde ich schade.

Dies gilt im übrigen für alle $S E_{1}$, auch für die des Typs "mit fakultativem Korrelat". Es schien uns jedoch geboten, diejenigen Fälle durch einen Verweis auf die Einlei- tung auszugliedern, in denen ein Vermerk

"mit obligatorischem Korrelat" nur bei einer "normalen" Satzgliedfolge gerechtfertigt gewesen wäre.

6.4. SE-Form

6.4.1. Außer nach SE-Klassen und SE-TYpen können SE auch nach ihrer (Ausdrucks-) Form unterschieden werden. Die SE-Form wird im KVL direkt vor dem Beispielsatz angegeben; dabei stehen

INF+ für Infinitivsatz mit $z u$ 。 INF- für Infinitivsatz ohne zu, DASS für daß-Satz, FRAG für indirekten Fragesatz und HPTS für hauptsatzförmige Ergänzung.

\subsubsection{Beispiele für INF+ sind etwa:}

Sie lehnt es $a b$, von ihm unterstutzt zu werden. Ir baut fest darauf, einen Kredit zu bekommen.

Wir haben uns im KVL darauf beschränkt, die üblichen und nicht alle möglichen Infinitivsätze mit zu zu erwähnen. 
6.4.3. Für Infinitivsätze ohne zu (INF-) gibt es nur wentge Belege; sie stehen vor allem bel verben der sinnlichen wahrnehmung: 1

Ich hörte den Marn kommen.

Ich fithte mein Herz schlagen.

Die SE in Infinitivform dürfen nicht verwechselt werden mit Dependentien von Modalund Modalitätsverben. ${ }^{2}$

6.4.4. Die SE-Form, die sich am häufigsten Im KVL findet, ist der daß-satz:

Er baut fest darauf, dảß sein Freund ver schwiegen ist.

Ex hat 〈es〉 nie erfahren, daß sein Freund ihn betrogen hat.

Nicht berücksichtigt ist im KVL, daß bei manchen Verben, besonders den verba sentiendi, ein daß-Satz mit einem wie-Satz kommutieren kann, wobei sich allerdings die Bedeutung leicht verändert:

1 Vgl. 5.2.2.

2 vgl. 4.6.
Ich sehe, daß er konmt.

Ich sehe, wie ex kommt.

Ebenso ist nicht verzeichnet, daß bei einigen Verben der Gemitsbewegung der daß- Satz gegen einen wenn- Satz austauschbar 1st:

Es freut mich, daß du kommst.

Es freut mich, wenn du konnst.

Auch hier sind Bedeutungsunterschiede festzustellen. Der wen-Satz zeigt allerdings ein anderes syntaktisches Verhalten als der daß-Satz; so wird z.B. es auch dann gesetzt, wenn der wenn-Satz im Vorfeld steht:

Wenn du kommst, freut es mich.

*Daß du korrnst, freut es mich.

Das würde u.a. dafür sprechen; den wennSatz als Angabesatz einzustufen. Da also sein status nicht ohne weiteres geklärt werden kann, haben wir ihn nicht ins KVL aufgenommen.

6.4.5. Eine weitere SE-Form ist FRAG, der indirekte Fragesatz. Darunter verste- 
hen wir alle die untergeordneten Sätze,

- die, in Hauptsatzform gebracht, eine Entscheidungs- oder $\omega$-Frage ergeben. Es

handelt sich also um ob-oder w-Sätze, wobei die letzteren keinesfalls mit den generalisierenden Ergänzungssätzen ${ }^{1}$ verwechselt werden dürfen:

Die Kinder versuchen, ob der Kuchen gut ist. Er konnte sich nicht besinnen, wo er den Brief hingelegt hatte.

Ich kann 〈es〉 mir schon denken, warum du nicht kommen willst.

Ich werde $\langle e s\rangle$ mir nie merken, wie dein Freund heiBt.

Nicht bei allen Verben, bei denen eine SE in der Form FRAG vorkommen kann, ist ein ob-Satz möglich. Deshalb ist eine Subklassifizierung der SE-Form FRAG ernsthaft in Erwägung zu ziehen. Bei den in dieser Kategorie aufgeführten wie-sätzen haben wir uns bemüht, solche auszuwählen, die nicht mit daß-sätzen kommutieren (s.o.).

1 Vgl. 6.1.2.
Die genannten Interrelationen ${ }^{2}$ zwischen Ergänzungen betreffen vor allem die Selektion der beiden SE-Formen DASS und FRAG. 6.4.6. Schlièlich bleibt noch die hauptsatzförmige Ergänzung (HPTS) zu nennen. Sie zeichnet sich aus durch Fehlen eines subjunktors und durch die im konstativen Hauptsatz ${ }^{3}$ übliche Satzgliedfolge, d.h. Zweitstellung des finiten Verbs, das übrigens häufig im Konjunktiv steht, besonders bei Verben des

\section{Sagens :}

Der Junge bestellt mir, ich solle in die stadt fahren.

Ich hoffe, du schreibst mir wieder.

Ich sage 〈es〉 immer wieder, es muß bald etwas geschehen.

2 Vgl. 6.1.4.

3 Vereinzelt findet sich auch die im interrogativen Hauptsatz ohne Fragewort übliche Satzgliedfolge, d.h. Erststellung des finiten Verbs: Ich weiß nicht, sind diese Leute noch bei Sinnen. $\mathrm{Da}$ solche Konstruktionen jedoch weniger gelăufig sind, haben wir sie nicht ins KVL aufgenommen. Im übrigen kommutieren sie immer mit einem ob-Satz: Ich weiß nicht, ob diese Leute noch bei Sinnen sind. 
Er bittet seinen Vater (darum), er möchte ihm helfen.

Wenn das fakultative Korrelat realisiert ist, könnte man - vorausgesetzt, das Verb des zweiten Satzes steht nicht im Konjunktiv I, - diese Konstrukte allerdings auch als zwei aneinandergereihte Hauptsätze betrachten, wobei dann das Korrelat als Anapher und damit als Ergänzung gewertet werden müßte. Die Tatsache, daß das Korrelat ohne weiteres wegfallen kann, rechtfertigt jedoch die Entscheidung, hier von éiner hauptsatzförmigen Ergänzung zu sprechen, die wie jede andere Ergänzung eine vom Verb eröffnete Leerstelle besetzt.

6.4.7. Abschließend ist noch auf eines besonders hinzuweisen. Alle hier genannten Formen, Infinitivsatz mit und ohne zu, indirekter Fragesatz und hauptsatzförmige Ergänzung, finden sich nicht nur als Realisierungsmöglichkeiten von $\mathrm{SE}$, sondern auch von $E_{9}$. Bei letzterem kann jedoch der Satz, gleich in welcher Form er auftritt, nicht durch eine."einfache" Ergänzung ersetzt werden: 1

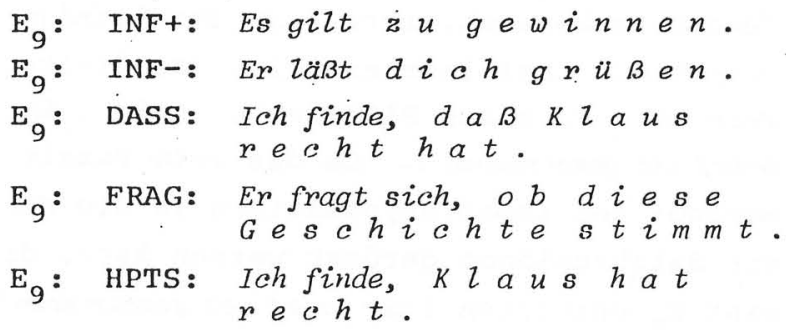

7. Passivfähigkeit

7.1. Formen des Passivs

Wenn bisher von Verbalsätzen die Rede war, so waren immer Aktivsätze gemeint. Im folgenden steht das Passiv im Mittelpunkt. Bekanntlich gibt es verschiedene Formen des Passivs. Im allgemeinen spricht man von einem werden- Passiv (oder Vorgangspassiv) und einem sein- Passiv (oder $\mathrm{Zu}-$ standspassiv). Bei ersterem wird eine Form

1 s. 6.1 .3 . 
des Hilfsverbs werden mit der unflektierten Form des Partizip Perfekt des betreffenden Verbs kombiniert ( der Brief wird geschrieben): bei letzterem ist das finite Verb eine Form des Hilfsverbs sein ( der Brief ist geschrieben). Da das sein-Passiv morpho- und semantosyntaktisch in die Nähe von Satzbauplänen gerückt werden kann, die eine $\mathrm{E}_{8}$ enthalten (der Brief ist geschrieben/ fertig/frankiert...), und da es zumindest immer dann stehen kann, wenn ein volles werden- Passiv möglich ist, bleibt es im Valenzlexikon unberücksichtigt. Außerdem bleiben unberücksichtigt "passivähnliche" Konstruktionen, wie sie im Kapitel 4.8.2. erwähnt worden sind.

\subsection{Angaben zur Passivfähigkeit}

Daß Im Valenzlexikon Hinweise zum Passiv。 genauer gesagt zum werden-Passiv, gegeben werden, hat im wesentlichen zwei Gründe. Zum einen erlaubt die Erwähnung der Passivfähigkeit von Verben, einen Satzbau- plan weiter zu spezifizieren:

$$
\begin{gathered}
\text { abgeben SBP 01. P1: } \begin{array}{l}
\text { Das Buch wurde recht- } \\
\text { zeitig von dem Schü- } \\
\text { Zer abgegeben. }
\end{array} \\
\text { abgeben SBP 01 PO: " } \begin{array}{l}
\text { Ein guter Ehemann wird } \\
\text { von Hans abgegeben. }
\end{array}
\end{gathered}
$$

Bekanntlich sind nicht alle Verben passivfähig, nicht einmal alle Akkusativverben, was semantische Gründe hat, auf die hier nicht weiter eingegangen werden kann ( Ich bekomme einen Brief. - "Ein Brief wird von mir bekommen. ). Die Passivfähigkeit ist also Kennzeichen nur einer Subklasse von Verben, die sich außerdem noch danach unterscheiden lassen, ob sie ein volles Passiv (markiert als P1) oder nur ein unpersönliches Passiv (markiert als P2) erlauben. Verben, die üblicherweise nicht im Passiv verwendet werden, tragen die Markierung $\mathrm{PO}$.

Zum anderen scheint es uns im Interesse der Benutzer geboten, möglichst viele verbspezifische Informationen $\mathrm{zu}$ geben, besonders wenn sie sich, wie das Passiv, unter dem Ge- 
sichtspunkt der Valenz verstehen lassen.

\subsection{Volles Passiv}

Beim vollen Passiv (P1) wird die $E_{1}$ des Aktivsatzes zur $\mathrm{E}_{\mathrm{O}}$ (zum "Subjekt" des Passivsatzesi. Die $\mathrm{E}_{\mathrm{O}}$ des Aktivsatzes wird im Passivsatz fakultativ; wenn sie aktualisiert wird, dann meist in Form einer Präpositionalphrase mit der Präposition von oder durch. Besonders wenn der. Satzbauplan eine weitere Ergänzung mit der Präposition von aufweist, wird durch gesetzt, um Ambiguitäten zu vermeiden:

Gritli grüste mich von seinem Vater.

Ich wurde von/durch Gritli von seinem Vater gegrilßt.

Die $\mathrm{E}_{\mathrm{O}}$ des Aktivsatzes kann im Passivsatz auch in bestimmten Fällen durch ein Adjektiv repräsentiert werden ( Das Gesetz ver bot damals die Tötung auf Verlangen. - Die Tötung auf Verlangen wurde damals gesetzlich verboten. ). Schließlich ist bei reziproken Verben ${ }^{1}$ im

$1 \mathrm{Vgl} .4 .4 .6$
Passiv eine Präpositionalphrase mit zwischen möglich:

Beide Parteien verabredeten ein erneutes Gespräch.

Wischen beiden Parteien wurde ein erneutes Gespräch verabredet.

Die hier angedeuteten Regeln der Passivkonverse gelten nicht für den Fall, daß ein partim reflexives Verb vorliegt ${ }^{2}$, wobei die zugehörige $E_{1}$ als Reflexivpronomen auftritt. Volles Passiv ist hier nicht möglich, nur unpersönliches (s.u.) (Peter wäscht sich. - "Er wird von Peter gewaschen; die resultierende Form ist zwar nicht ungrammatisch, aber eine Konverse zu Peter wäscht ihn. ).

Bei Verben, die zwar mit P1 markiert sind, deren E1 aber fakultativ ist, ist natürlich nur unpersönliches Passiv möglich, wenn die E1 nicht aktualisiert ist:

Manchmal kauft Thomas ein.

2 Vgl. 4.4.4. 
Manchmal wird von Thomas eingekauft.

\subsection{Unpersönliches Passiv}

Das unpersönliche Passiv (P2) findet sich bei intransitiven Verben, bei Verben also, deren Satzbauplan keine $\mathrm{E}_{1}$ aufweist. Demnach kann es in der Passivkonverse keine $E_{0}$ geben, die sich aus einer $E_{1}$ im Aktivsatz hèrleiten ließe. Deshalb tritt ins Vorfeld des finiten Verbs, wenn es nicht anderweitig besetzt ist, das nicht austauschbare es. Im übri'gen gelten die gleichen Regeln wie für das volle Passiv ( Der Rechtsanwalt geht gegen die. Entscheidung des Gerichts an. - Von dem Rechtsanwalt wird gegen ... angegangen. / Es wird von dem Rechtsanwalt gegen ... angegangen. ).

Abschließend ist noch eine Einschränkung beim unpersönlichen Passiv zu erwähnen. Bereits im Zusammenhang mit dem vollen Passiv ist darauf hingewiesen worden, daß die aus der $\mathrm{E}_{\mathrm{O}}$ des Aktivsatzes entstandene von- Phrase in der Passivkonverse fa- kultativ wird. Beim unpersönlichen Passiv läßt sich sogar feststellen, daß die Sätze mit von-Phrasen weniger üblich sind als die ohne ( es wird getanzt - es wird jetzt getanzt von euch.).

8. Beispielsätze und Bemerkungen

8.1. Problematik der Beispiele

Die Beispielsätze haben in erster Linie die Funktion, die angegebenen grammatischen Strukturen zu illustrieren. Darüber hinaus haben wir versucht, Sätze zu finden, die möglichst in der. Praxis anwendbar sind und deren Wortmaterial in der Regel aus der Wortliste des zertifikats 'DaF' stammt.' Auf diese Weise soll gewährleistet werden, daß die Beispiele den Sprachkenntnissen. der Zielgruppe angemessen sind. Allerdings mußte öfters über den Wortbestand der Zertifikatsliste hinausgegangen werden, weil besonders durch den Mangel an abstrakten

1 S. 1.2 . 
Nomina für manche Satzbaupläne sonst kaum akzeptable Belege zu finden gewesen wären. Grundsätzlich stellt sich das problem, daß die erlernten strukturen in Texten verwendet werden sollen, während es im Lexikon nur möglich ist, Beispiele auf Satzrang zu geben, die ohne weitergehenden Kontext verständlich sein sollen. Diese Diskrepanz ist nicht, wie oft behauptet wird, dadurch zu beheben, daß man als Beispiele nur Sequenzen zuläßt, die in einem wie auch immer gearteten Corpus belegt sind. Abgesehen davon, daß man zur Auffindung der selten auftretenden strukturen ein riesiges Corpus analysieren müßte, sind die aus dem Textzusammenhang herausgelösten Originalbelege meistens in der Praxis nicht besser verwendbar als konstruierte Sätze. Wo es zweckmäßig war, haben wir allerdings auf die Ergebnisse unserer Corpusanalyse auch für die Beispielsätze zurückgegriffen. Auf andere Probleme der Beispielsätze wird im Forschungsbe- richt 'Untersuchungen zur Verbvalenz' eingegangen. 1

\subsection{Form der Beispiele}

Die Beispielsätze werden überwiegend in einer Standardform gegeben, d.h. als einfache aktivische Aussagesätze im Präsens, Indikativ, die möglichst keine Angaben und Attribute enthalten. Diese Form ist zwar geeignet, um die Grundstruktur des Satzes sichtbar zu machen, führt jedoch in manchen Fällen zu wenig akzeptablen Sätzen. Einige Beispiele sind fast ausschließlich mit Verbangaben möglich. Dies gilt besonders, wenn die fakultativen Ergänzungen fehlen. Im Satz Er hilft seiner Frau bei der Ar beit. können bis auf die $E_{0}$ alle Ergänzungen weggelassen werden. Wenn sie jedoch fehlen, steht z.B. eine Lokalangabe:

Er hilft im Haushalt.

Da in den Beispielsätzen fakultative Ergän-

1 Vgl. Schumacher, Zum Forschungsbericht, S. 10-12. 
zungen aktualisiert werden, braucht man in diesem Fall keine Angaben hinzuzusetzen. Wenn hingegen selbst bei der Realisierung aller Ergänzungen wentg informative und ungebräuchliche Sätze entstehen, enthält der Beisplelsatz auch Angaben.

Einige Verben z.B. werden (fast) nur mit der Negation gebraucht:

Ich komme nicht umhin, dich tadeln zu müssen.

Ein anderer Grund für die Aufnahme von Angaben ișt die Möglichkeit, dadurch mitunter verschiedene Verbbedeutungen deutlich zu machen, wenn der Satzbauplan gleich ist:

Der Vogel fliegt (nach Süden).

Hans fliegt [im Jumbo-Jet] (nach Mailand).

Es hat sich auch gezeigt, daß die durch die standardisierung bedingte Form des Verbalkomplexes bei einigen Verben nicht der üblichen.Verwendung entspricht. Er finden im Sinne von eine Erfindung machen kommt oft in Verbindung mit den Vergangenheitstempora vor:
Wankel erfand einen Motor.

Wertpopiere handeln steht dazu immer im Passiv. Andere Verben erscheinen gewöhnlich im Konjunktiv oder in Kombination mit einem Modalverb:

Ich kann mir gut vorstellen, daß mein Vater kommen wird.

Einem verschiedentlich von Fremdsprachendidaktikern geäußerten Rat folgend, haben wir uns dazu entschlossen, manchmal von der Standardform abzugehen, um möglichst sätze $\mathrm{zu}$ bekommen, die im Unterricht als Beispiele verwendbar sind.

8.3. Arten von Beispielsätzen

Grundsätzlich wird jeder SBP durch einen Beispielsatz illustriert. Mehrere Sätze werden in den Fällen angegeben, in denen deutlich unterschiedliche Verbbedeutungen nicht durch verschiedene Satzbaupläne, unterschiedliche Formen von SE oder Unterschiede in der Passivbildung separiert werden können: 
Das Licht brennt.

Euer Haus brennt.

Da diesem Lexikon keine explizite Semantiktheorie zugrundeliegt, sind die Trennungen nicht theoretisch motiviert.

Bei vielen Verben sind in bestimmten SBP Pertinenzelemente möglich. ${ }^{1}$ Der entsprechende Vermerk im wörterbuch wird durch einen eigenen Beispielsatz erläutert. In einigen Fällen enthält auch bereits das - SBP-Beispiel einen Pertinenzdativ, wenn diese Verben kaum Verwendungsweisen haben, in denen ein Pertinenzelement ausgeschlossen wäre:

Cäsar küßt seine Freundin auf die Stim.

Bei den Ausbauplänen wird jede mögliche SE-Form durch einen Satz belegt.

8.4. Codierung der Beispielstze

Die Beispielsätze sind so notiert worden, daß jede im SBP aufgezählte Ergänzungs-

1 s. 5.2 .3 . und 5.4 .4 . klasse aktualisiert wird. Soweit es die Regeln der Satzgliedfolge im Deutschen zulassen - was meist der Fall ist - entspricht die Abfolge der Satzglieder im Beispiel der Reihenfolge dér Codeziffern im SBP-Code.

Um Mißverständnisse $\mathrm{zu}$ vermeiden, werden auch im SBP-Beispiel entsprechend der Notation im Code obligatorische Ergänzungen nicht markiert, während fakulțative Ergänzungen in $\mathrm{R} \mathrm{u} \mathrm{n} \mathrm{d} \mathrm{k} l \mathrm{a} \mathrm{m} \mathrm{m} \mathrm{e} r \mathrm{n}$ erscheinen. Auf diese Klammerung ist in den Beispielen $z$ u den SE verzichtet worden, da bei der Einklammerung von Nebensätzen die Notation nicht mehr übersichtlich ist. Ebenfalls aus Gründen.der Ubersichtlichkeit werden bei SBP mit disjunk-. ter Besetzung von Leerstellen drei Beispiele angegeben, die den drei möglichen Aktualisierungsformen entsprechen. Der erste Satz illustriert die Maximalform mit der Besetzung beider Positionen. Im zweiten Beispiel wird allein das jeweils 
erste Element des Disjunktionspaares aktualisiert, während im dritten satz nur das zweite Element auftritt. Beim Verb schreiben im SBP 01v3 lauten dementsprechend die Beispiele:

Er schreibt seiner Mutter einen Brief.

Er schreibt einen Brief.

Er schreibt seiner Mutter.

In e c k $\mathrm{i} g$ e $k \mathrm{l}$ a m e $\mathrm{m}$ werden in den Beispielsätzen zum SBP die Verbangaben gesetzt. Die gelegentlich vorkommenden Attribute bleiben dagegen unmarkiert.

Schließlich ist noch die $\mathrm{s} p \mathrm{i}$ t $\mathrm{z} \mathrm{l}$ a $\mathrm{m}-$ $m$ e $r$ zu nennen, die eine doppelte Funktion hat. Sie kennzeichnet einmal das fakultative Reflexivpronomen. Außerdem wird das fakultative Korrelat in den Beispielen zum Ausbauplan in Spitzklammern gesetzt.

8.5. Bemerkungen im Wörterbuch

Einige Wörterbuchartikel werden durch eine Bemerkung abgeschlossen. In der linken
Spalte unter dem Verbkomplex etwas eingerückt steht der Eintrag: BEMERKUNG. Der Text der Bemerkung ist rechts, im Verhältnis zu den Beispielsätzen etwas eingerückt, placiert.

Ein Typ von Bemerkungen bezieht sich auf die stammformen der Verben. Wenn es gleichlautende Verben mit starker und schwacher Flexion oder mit Perfektbildung mit haben und sein gibt, werden die wörterbuchartikel getrennt und die stammformen in einer Bemerkung angegeben.

Die Besonderheiten der Perfektbildung bei den reflexiven und reziproken Verben ${ }^{1}$ werden in den Bemerkungen nicht berücksichtigt. Eine andere Form von Bemerkungen kann nach dem Satzbauplan stehen und bestimmte Informationen zur Besetzung von einzelnen Ergänzungspositionen enthalten. Oft beziehen sich diese Angaben auf die $E_{1}$ und besagen, $\mathrm{da} \beta$ bei dieser Struktur die Besetzung meist indefinit ist oder daß normalerweise eine SE vorkommt.

1 Vgl. Helbig/Buscha, Grammatik, S. 116. 
9. Maschinenausdruckezu Valenzlexikon und $V$ alenzregister

\subsection{Valenzlexikon}

Bei der Erstellung des Valenzlexikons haben wir uns von vornherein der Mittel der maschinellen Datenverarbeitung bedient. Alle Informationen wurden auf Lochkarten übertragen und maschinell ausgewertet. Es gibt daher eine ganze Anzahl von Maschinenausdrucken zu diesem Lexikon, die nach verschiedenen Auswahl- und Sortierkriterien organisiert sind. ${ }^{1}$ Eine genauere Beschreibung enthält der Beitrag von HAMEL und SCHUMACHER im Forschungsbericht "Untersuchungen zur Verbvalenz".2

Neben der Standardversion, die mit einigen Ausnahmen der hier vorliegenden Buchfassung

1 Die Maschinenprogramme wurden am Datenverarbeitungszentrum des IdS von $J$. Lindemann und A. Kurbel erstellt. Bei der Aufbereitung der Daten für die maschinelle Auswertung wirkten $\mathrm{K}$. Keller und $\mathrm{G}$. Wettstein mit.

2 Vgl. Hamel/Schumacher, Maschinenausdrucke. entspricht, gibt es einen Ausdruck, in dem jeweils die Verben mit einem bestimmten Satzmuster zusammengefaBt werden. Dazu wird auch eine statistik über die Häufigkeit des Vorkommens der einzelnen Satzmuster gegeben. In einer anderen Gruppe von Ausdrucken werden nur die wörterbucheinheiten herausgegriffen, die mit einer satzförmigen Ergänzung auftreten können. Diese Einheiten können dann nach verschiedenen Gesichtspunkten sortiert werden, nämlich nach den E-Klassen, bei denen eine SE auftritt, oder nach den füf Formen der Nebensätze sowie nach dem Kriterium, ob ein Korrelat auftritt oder nicht. Diese Sortierbegriffe können auch kombiniert werden, indem etwa innerhalb jeder E-Klasse noch nach dem Vorkommen des Korrelats unterschieden wird. Innerhalb der aussortierten Gruppe erscheinen die Verben dann wieder alphabetisch. Spezielle Auswahlregister gibt es auch für die Reflexivverben und die lexikalischen Fügungen. 
Diese Spezialregister dienen in erster Inie als Materialbasis für linguistische Untersuchungen der verschiedensten Art. Für manche Aufgabenstellungen hat es sich als zweckmäßig erwiesen, wenn die Daten nicht im fortlaufenden Maschinenausdruck stehen, sondern jede Wörterbucheinheit auf einer Karteikarte einzeln ausgedruckt wird. Damit hat jeder Benutzer die Möglichkeit, sich sein Material nach anderen Ordnungskriteriēn zusammenzustellen. Wir haben deshalb die Möglichkeit geschaffen, alle versionen auch in dieser Form auszudrucken. Nach der Fertigstellung·des Erweiterten Valenzlexikons werden auch für das größere Arbeitsvorhảben die entsprechenden Spezialregister vorliegen. Diese-können bei der Projektgruppe 'Verbvalenz' am Institut für deutsche Sprache in Mannheim von Linguisten für wissenschaftliche Zwecke bezogen werden.

\subsection{Valenzregister}

Auch für das Teilprojekt 'Valenzregister' werden die Analysedaten maschinell ausgewertet. ${ }^{1}$ Bei diesem Unternehmen werden durch ein statistisches Verfahren ausgewählte Textmengen aus dem Mannheimer Corpus morphosyntaktisch analysiert. ${ }^{2}$ Es können Häufigkeitsstatistikén über die auftretenden Satzbaupläne sowie über eine Anzahl von anderen syntaktischen Kategorien erstellt werden.

Bei der Standardversion werden die analysierten Einheiten fortlaufend nach ihrem Vorkommen im Text ausgédruckt: Statistische Auswertungen sowohl nach den Einzeltextmengen als auch nach den Textgattungen schließen sich an. Ein zweites Register sortiert die-Daten nach 'Satzmustern, während in der dritten Version die auftre-

1 Die Maschinenprogramme wurden von 0 . Winkelmann, Universität Mannheim, erstellt.

2 Vgl. Schumacher/Höfert, Erläuterungen. 
tenden regierenden Verben als oberster Sortierbegriff dienen.

Es ist ferner vorgesehen, spezielle Auswahlregister, z.B. für satzförmige Ergänzungen, Pertinenzelemente, Passiversatzformen und bestimmte Informationen zum Auxiliarkomplex, $z u$ erstellen. $\mathrm{zu}$ den Analysedaten kann auch jewells der Belegsatz aus dem Corpus maschinell zugeordnet werden. Neben den fortlaufenden Maschinenausdrucken können auch bei diesem Projekt alle Bearbeitungseinheiten im Datenformat der verschiedenen Versionen einzeln auf Karteikarten erstellt werden. Nach $\mathrm{Ab}-$ schluB der Arbeiten können interessierte Sprachwissenschaftler die Ausdrucke über die Projektgruppe 'Verbvalenz' bestellen. 



\section{Litcraturverzeichnis}

Axbeitsgruppe Mas $\Lambda$, Zur maschinellen Syntaxanalyse I. Morphosyntaktische Voraussetzungen für eine maschinelle Sprachanalyse des Deutschen, Tubingen 1974,2 Teile (= IdS-Forschungsberichte 18.1. und 18.2.). ( $\mathrm{s} y \mathrm{n}$ t a $\mathrm{x}$ a $\mathrm{n}$ a $I$ y $\mathrm{s}$ e I )

-- . Zur maschinellen syntaxanalyse II. Ein Lexikon für eine maschinelle Sprachanalyse des Deutschen, Tubingen 1974 (= IdS-Forschungsberichte 19). ( $S$ y $n$ t a $x$ a n a 1 y s e I I )

Ballweg, Joachim, $1: \mathrm{SE}$ mit fakultativem Korrelat, in: Schumacher, Helmut

(Hrsg.), Untersuchungen, S. 248-252. ( $1: S E$ m $i t$ a $k$ u 1 a $t i$ $\mathrm{v}$ e $\mathrm{m}$ K o r r e l a $t$ )

---, Zur Diskussion des syntaktischen status der Präpositionalphrasen des Týps Hans trifft das Fenster mit dem Stein, in: Schumacher, Helmut (Hrsg.), Untersuchungen, S. 253-258. ( H a ns $t r i f f t$ das renster)

Ballweg-Schramm, Angelika, Korrelat und Satzgliedstellung, dargestellt am Beispiel satzförmiger $\mathrm{E}_{\text {ond }} \mathrm{E}_{\text {, }}$ in: Schumacher, Helmue (Hrsg.), Untersuchungen, S. 240-247. ( K O r r e 1 a $t$ u n d $s$ a $t z g l i$ e d s t e l l l u n g )
Baltruweit, Sabine, Analyse fremdsprachlicher Curricula in der Weiterbildung, in: Pädagogische Arbeitsstelle des DVV (Hrsg.), Materialien zu den VHS-Zertifikaten Nr. 24, Frankfurt/M. 1975. ( A n a 1 y s e)

Biere, Bernd Ulrich, Ergänzungen und Angaben, in: Schumacher, Helmut (Hrsg.), Untersuchungen, S. 129-173. ( E r $\mathrm{g} \ddot{a} \mathrm{n} z$ u $n \mathrm{~g}$ e $\mathrm{n}$ )

Brinkmann, Hennig, Die deutsche Sprache. Gestalt und Leistung, Düsseldorf 1962, 2. Aufl. 1971. ( D e u t s c h e s p r a c h e)

Brosch, Gerd, Das Zertifikat Deutsch als Fremdsprache, in: Wierlacher, Alois (Hrsig.), Jahrbuch Deutsch als Fremdsprache, Band 1, Heidelberg 1975, s. 149-152. ( $z$ e $r$ t $i f i k$ a $t$ D a F )

Buhler, Karl, Sprachtheorie, Jena 1934, 2. Aufl. Stuttgart 1965. ( $s$ p r a c h theorie)

Enge1, Ulrich, Die Satzbaupläne der deutschen Gegenwartssprache I $u$. II, in: der deutsche Lehrer im Ausland, Heft 10, 1970, S. 259-268 u. Heft 11, 1970, S. 294-302. ( $S$ a $t z$ b a u p 1 ä$\mathrm{n}$ e )

---, Die deutschen Satzbaupläne, in: Wirkendes Wort 20, 1970, S. 361-392.

( $D$ e u $t$ s c h e $s$ a $t z$ b a $u$ p 1 ä $n$ e ) 
Engel, Ulrich, Bericht uber das Forschungsunternehmen "Grundstrukturen der deutschen Sprache", in: Moser, Hugo (Hrsg.), Sprache und Gesellschaft. Beiträge zur soziolinguistischen Beschreibung der deutschen Gegenwartssprache, Jahrbuch 1970 des IdS, Düsseldorf 1971, S. 295322 (= Sprache der Gegenwart 13). ( $\mathrm{G}$ u n d s $\mathrm{t}$ u k t u r e n)

---, Thesen zur Syntax, in: Bulletin Phonographique 12, 1971, S. 85-107. $(\mathrm{T} h$ e $\mathrm{s}$ e $\mathrm{n}$ )

---, Bemerkungen zur Dependenzgrammatik, in: Moser, Hugo (Hrsg.), Neue Grammatiktheorien, S. 111-155. ( B e $\mathrm{m}$ e $\mathrm{r} k \mathrm{u} \mathrm{n} \mathrm{g}$ e $\mathrm{n}$ )

---, Syntax der deutschen Gegenwartssprache, Berlin 1977 (= Grundlagen der Germanistik 22). (

Engelen, Bernhard, Untersuchungen zu Satzbauplan und wortfeld in der geschriebenen deutschen Sprache der Gegenwart, München 1975, 2 Bde. (= Heutiges Deutsch, Reihe 1, Bd. 3). ( U n $t$ e r.s u c h u n g e n )

Erben, Johannes, Deutsche Grammatik. Ein Abriß, 11., völlig neu bearbeitete Auflage von "AbriB der deutschen Grammatik", München 1972. ( G r a m m a t i k )
Flämig, Walter u.a., Skizze der deutschen Grammatik, Berlin 1972. ( S k i z z e )

Glinz, Hans, Die innere Form des Deutschen, 4. Auf1. Bern, München 1965, 5. Aufl. 1968 .

( I $\mathrm{n} n$ e $\mathrm{r}$ e $\mathrm{F}$ or $\mathrm{m}$ )

Grebe, Paul (Hrsg.), Duden-Grammatik der deutschen Gegenwartssprache, 3., neu bearbeitete und erweiterte Auf1., Mannheim 1973 (= Der Große Duden, Bd. 4). ( D u d e n - G r a m $\mathrm{m}$ a $\mathrm{t} i \mathrm{k}$ )

Günther, Heide, Zur Codierung verbabhängiger infiniter Verbalsyntagmen, IdS-Arbeitspapier V 102 der Arbeitsgruppe 'Valenzlexikon', Mannheim 1972 (vervielfältigt).. ( $\mathrm{C}$ o d $\mathrm{i}$ e $\mathrm{r}$ u $\mathrm{g}$ )

---, Zur Korrektur der Infinitivkodierung im Valenzlexikon, IdS-Arbeitspapier V 107 der Arbeitsgruppe 'Valenzlexikon', Mannheim 1972 (vervielfältigt). ( $\mathrm{k} \circ \mathrm{r}$ e k $\mathrm{t} u \mathrm{r}$ )

Günther, Heide/Pape, Sabine, Funktionsverbgefüge als Problem der Beschreibung komplexer Verben in der Valenztheorie, in: Schumacher, Helmut (Hrsg.), Untersuchungen, $S .92-128$. ( $\mathrm{F}$ u n k $t i \circ \mathrm{s} v$ e $r$ b g e $f \ddot{u} g$ e)

Hamel, Günther/Schumacher, Helmut, Die Maschinenausdrucke des Mannheimer Valenzlexikons, in: Schumacher, Helmut (Hrsg.), Untersuchungen, S. 293-313. ( $M$ a s c hi n e n a s d r u c k e) 
Helbig, Gerhard, Valenz und Tiefenstruktur, in: Deutsch als Fremdsprache 6, 1969, S. 159-169. ( V a 1 e $n$ z u n d $\mathrm{T} i \mathrm{e}$ e $\mathrm{n} \mathbf{s} \mathrm{r}$ u k $\mathrm{t}$ u r

---, Valenz, Tiefenstruktur und Semantik, in: Glottodidactica 3/4, 1969, S. 1146. $(\mathrm{V}$ a 1 e $\mathrm{n} z)$

Helbig, Gerhard/Buscha, Joachim, Deutsche Grammatik, 2. Aufi. Leipzig 1974.

( G $r$ a $\mathrm{m} \mathrm{m}$ a $t i k$ )

Helbig, Gerhard/Schenkel, Wolfgang, Wörterbuch zur Valenz und Distribution deutscher Verben, Leipzig 1969, 2., uberarbeitete und erweiterte Aufl., Leipzig 1973, 3. Aufl. Leipzig 1975. ( W ör t e r b u c h r

Heringer, Hans-Jürgen, Theorie der deutschen Syntax, München 1970, 2. Auf1. München 1973 (= Linguistische Reihe 1).

( $\mathrm{T}$ h e o r i e)

Kaufmann, Gerhard, Grammatik der deutschen Grundwortarten, München 1967 (= Schriften der wissenschaftlichen Arbeitsstelle des Goethe-Instituts 1). ( G r a m m a $\mathrm{t} 1 \mathrm{k}$ )

Keim, Inken/Volkmann, Barbara, Die Reflexiva, IdS-Arbeitspapier V 52 A der Arbeitsgruppe 'Valenzlexikon', Mannheim 1972 (vervielfältigt). ( $\mathrm{R}$ e $\mathrm{f} 1$ e $\mathrm{x} \perp \mathrm{v}$ a)

Köhler, Karl-Heinz, Zum Problem der Korrelate in Gliedsätzen, in: Schumacher,
Helmut (Hrsg.), Untersuchungen, S. 174239. ( $\mathrm{k} \circ \mathrm{r}$ e 1 a $t$ e $i \mathrm{n}$

G $l i$ e d s ä $t z$ e $n$ l

Leirbukt, Oddleif, Vorschläge für eine operationelle Untersuchung als Vorarbeit fir die Unterscheidung zwischen Verbzusatz und Satzglied im Bereich der adjektivischen Elemente, in: Schumacher, Helmut (Hrsg.), Untersuchungen, S. 7591. ( V O r s c h 1 äg e )

Moser, Hugo (Hrsg.), Neue Grammatiktheorien und ihre Anwendung auf das heutige Deutsch, Jahrbuch 1971 des IdS, Düsseldorf 1972 (= Sprache der Gegenwart 20). ( $\mathrm{N} e \mathrm{u}$ e $\mathrm{G} \mathrm{r}$ a in $\mathrm{m}$ a $\mathrm{i} k \mathrm{t} \mathrm{h}$ e o$r$ i e n )

Neugeborn, Wolfgang, Zur Analyse von Sätzen mit finiter Verbform + Infinitiv, in: Schumacher, Helmut (Hrsg.), Untersuchungen, S. 66-74. ( $\mathrm{z}$ u r A n a l y $\mathrm{s}$ e )

Pädagogische Arbeitsstelle des DVV (Hrsg.), Erster Zwischenbericht der Gutachterkommission Lernziele. Zur Explizierung der Lernziele der VHS-Sprachenzertifikate, Frankfurt/M. 1974 (= Materialien $\mathrm{zu}$ den VHS-Zertifikaten Nr. 12). ( E r s t e r z w i $\mathrm{s} c \mathrm{~h}$ e $\mathrm{n} \mathrm{b}$ e $\mathrm{r}$ i c h t )

Pape, Sabine, Bemerkungen zu einigen Grundbegriffen der Valenztheorie, in: Schumacher, Helmut (Hrsg.), Untersuchungen, S. 21-53. ( $\mathrm{B}$ e m e $\mathrm{r}$ k n g e n ) 
Pape, Sabine, Stellungnahme zu verschiedenen Verbklassifikationen, in: Projektgruppe 'Verbvalenz', Jahresbericht 1975, S. 9/1-18. ( s t e 1 l u n $g \mathrm{n}$ a $\mathrm{h} \mathrm{m} e$ )

---, Zur Methodik der Ermittlung von Verbwortfeldern, in: Projektgruppe 'Verbvalenz', Jahresbericht 1975, s. 10/1-16. ( $\mathrm{z}$ u $r$ M e $t \mathrm{~h}$ o d i k )

v. Polenz, Peter, Der Pertinenzdativ und seine Satzbaupläne, in: Engel, Ulrich/ Grebe, Paul/Rupp, Heinz (Hrsg.), Festschrift für Hugo Moser zum 60. Geburtstag, Düsseldorf 1969, S. 146-17i. ( $P$ e $r t i n$ e $n$ z d $t i v$ )

Projektgruppe 'Verbvalenz', Erarbeitung einer Verbvalenzgrammatik auf semantischer Basis zur Vorbereitung eines Valenzwörterbuchs, Mannheim, Dezember 1975 (= Jahresbericht 1975). ( J a h r e s b e r i c h t 1976 )

Schenkel, Wolfgang, Die Valenz im adnominalen Raum, in: Helbig, Gerhard (Hrsg.), Beiträge zur Valenztheorie, The Hague, Paris 1971 , S. 67-83 (= Janua Linguarum, Series minor 115). ( V a $1 \mathrm{e} n \mathrm{z}$ )

Schulz, Dora/Griesbach, Heinz, Grammatik der deutschen Sprache, 3., überarbeitete Aufl., München 1965, 9., neubearbeitete Aufl., München 1972. ( $\mathrm{G}$ r a m a $\mathrm{mi} \mathrm{k}$ )
Schumacher, Helmut, Zum deutschen Valenzlexikon, in: Moser, Hugo (Hrsg.), Neue Grammatiktheorien, S. 184-193. ( $\mathrm{z}$ u m V a 1 e $\mathrm{n}$ z 1 e $\mathrm{x} i \mathrm{k}$ o $\mathrm{n}$ )

--- Ein deutsches Valenzlexikon, in: Nickel, Gerhard/Raasch, Albert (Hrsg.), IRAL-Sonderband, KongreBbericht der 3. Jahrestagung der GAL e.V., Heidelberg 1972, S. 237-242. ( $\mathrm{E}$ i $\mathrm{n}$ V a 1 e $n z l$ e $x i k \circ n$ )

---, Probleme der Verbvalenz, in: Werner, Otmar/Fritz, Gerd (Hrsg.), Deutsch als Fremdsprache und neuere Linguistik, München 1975, S. 41-66. ( P r o b 1 e $m$ e)

-- , Uber das Mannheimer Wörterbuch zur Verbvalenz, in: Zielsprache Deutsch, 1,1976 , S. 10-15.

--- (Hrsg.), Untersuchungen zur Verbvalenz. Eine Dokumentation über die Arbeit an einem deutschen Valenzlexikon, Tühingen 1976 (= IdS-Forschungsberichte 30). ( $u n t e r s$ u $\mathrm{c}$ u $\mathrm{n}$ g e $n$ )

---, Zum Forschungsbericht 'Untersuchungen zur Verbvalenz', in: Schumacher, Helmut (Hrsg.), Untersuchungen, S. 520. ( $\mathrm{z} u \mathrm{~m}$ F. $\circ \mathrm{r} \mathrm{s}$. h u n $\mathrm{g}$ $\mathrm{b} e \mathrm{r} i \mathrm{c} \mathrm{h}$ )

---, Valenz und Dependenz, Entwicklung und Forschungsstand (= Linguistische Arbeiten), Tübingen (erscheint 1978).

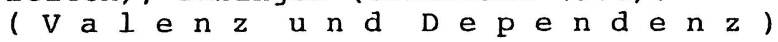


Schumacher, Helmut/Höfert, Günther, Erläuterungen $\mathrm{zu}$ den Maschinenausdrucken des Projekts 'Valenzregister', in: Projektgruppe 'Verbvalenz', Jahresbericht 1975, S. 27/1-27. ( E r 1 ä u t e r u n g e n)

Sommerfeldt, Karl-Ernst/Schreiber, Herbert, Untersuchungen zur syntaktischen und semantischen Valenz deutscher Adjektive (1) und (2), in: Deutsch als Fremdsprache 8, 1971, S. 227-231 und 287-293. ( $u n t$ e $r$ u c h u n $\mathrm{g}$ e $\mathrm{n}$ )

---, Wörterbuch zur Valenz und Distribution deutscher Adjektive, Leipzig 1974. ( $W$ ö $r$ e $r$ b u c h )

---, zu einem Wörterbuch der Valenz und Distribution der Substantive, in: Deutsch als Fremdsprache 12, 1975 , S. 112-119. ( $\mathrm{z} u$ e $i \mathrm{n}$ e $\mathrm{m}$ W ö $\mathrm{r}-$ t e $r$ b u c h )

Steger, Hugo (Hrsg.), Das Zertifikat 'Deutsch als Fremdsprache'. Für den Deutschen Volkshochschulverband e.V. Bonn und des Goethe-Instituts zur Pflege deutscher Sprache und Kultur im Ausland. Bonn 1972. ( $\mathrm{z}$ e $r \mathrm{t}$ i $\mathrm{i} k \mathrm{at}$ ' D a F ' )

Stötzel, Georg, Ausdrucksseite und Inhaltsseite der Sprache. Methodenkritische Studien am Beispiel der deutschen Reflexivverben, München 1970 (= Linguistische Reihe 3). ( A u s d r u c k s s e i $t$ e)
Tesnière, Lucien, Esquisse d'une syntaxe structurale, Paris 1953.

( $\mathrm{E}$ s q u i s $\mathrm{s}$ e)

--- Eléments de syntaxe structurale, Paris 1959, 2. Aufl. 1966. ( $\mathrm{E} l$ é $m$ e $n t s$ ) 


\section{Register}

Adjektiv

4.2 .3 5.5 .2 . 5.7 .4 .

$5 \cdot 7 \cdot 5$.

6.1 .4

Akkusativergänzung $\left(E_{1}\right)$

Akkusativ der Erstreckung

S. auch Pertinenzakkusativ

als-Phrase

5.7 .2 .

5.7 .5 .

als ob-Satz

5.8 .5 .

Anapher

Anaphorisierung

Angabe

Angabe vs. Ergänzung

Angabesatz

Artergänzung (Qualifikativergänzung) $\left(\mathrm{E}_{8}\right)$

6.3 .2 .

4.2 .3 .

4.3 .2 .

4.7 .

4.8 .4 .

5.7 .

5.8 .

2.4 .

$5 \cdot 6 \cdot 3$,

2.4 .

6.1 .1 . 6.4 .4 .
Artergänzung (Qualifikativergänzung) $\left(E_{8}\right)$

Attributsatz

Ausbauplan

Austauschbarkeit (Kommutierbarkeit)

4. 2.2

4. 4.2 .

4. 4.3 .

4.4 .4 .

4. 4.6 .

4. 5.1 .

4.7 .

4.8.5.

5.0 .

bei-Phrase

Beispielsatz

5.5 .1

6.4 .4 .

5.5 .3$.

8.1 .

8.2 .

8. 3 .

8.4 .

8.5 .

2.7 .

4.8 .5 .

5.5 .2 . 


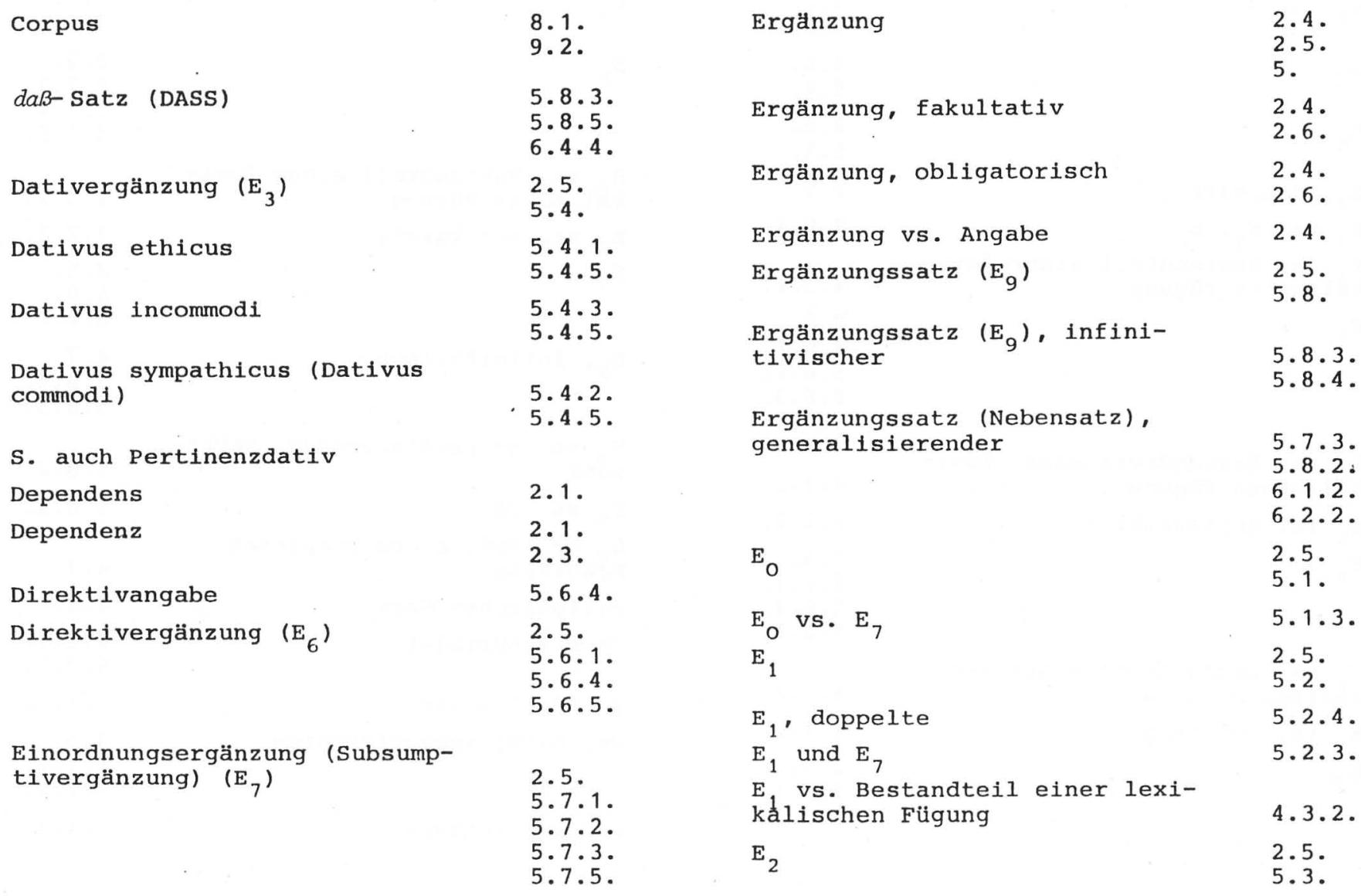


$\mathrm{E}_{2}$ vs. $\mathrm{E}_{4}$
$\mathrm{E}_{3}$
$\mathrm{E}_{4}$
$\mathrm{E}_{4}$, doppelte
$\mathrm{E}_{4}$ vs. $\mathrm{E}_{5}, \mathrm{E}_{6}$
$\mathrm{E}_{4}$ vs. Bestandteil einer lexi-
$\mathrm{kälischen} \mathrm{Fügung}$
$\mathrm{E}_{5}$

$E_{5}$ vs. Bestandteil einer lexikălischen Fügung

$E_{5}$ ys. Verbzusatz

$\mathrm{E}_{6}$

$E$ vs. Bestandteil einer lexikălischen Fügung

$\mathrm{E}_{6}$ vs. Verbzusatz

$\mathrm{E}_{7}$
5.3

$5.5 \cdot 6$.

2.5 .

5.4 .

2.5 .

5.5 .

5.5 .

5.5 .1 .

4.3.2.

2.5 .

5.6 .1 .

5.6 .2 .

$5 \cdot 6 \cdot 3$.

5.6 .5 .

4. 3.2:

4.2 .2 .

2.5 .

5.6 .1 .

5.6 .4 .

5.6 .5 .

4.3.2.

4.2 .2 .

2.5 .

5.7 .1 .

5.7 .2 .
$\mathrm{E}_{7}$

$\mathrm{E}_{8}$

$5 \cdot 7 \cdot 3$.

5.7 .5 .

2.5 .

5.7 .1 .

5.7 .4 .

5.7 .5 .

$E$ vs. Bestandteil einer lexikălischen Fügung

$\mathrm{E}_{8}$ vs. Verbzusatz

$\mathrm{E}_{9}$

$\mathrm{E}_{9}$, infinitivische

4.3 .2 .

4.2 .3 .

2.5 .

5.8 .

6.4 .7 .

4.7 .

5.8 .4 .

5.8 .5 .

$\mathrm{E}_{9}$ vs. generalisierender Nebensâtz

$\mathrm{E}_{9}$ vs. SE

5.8 .2 .

5.8 .2 .

$E_{g}$ vs. Teil eines komplexen

Prädikats

4. 7 .

elliptischer Satz

4.6 .

es als Korrelat

6.3 .1 .

6.3 .5 .

es, expletives

5.1 .2 .

es, nicht kommutierbares

4.5 .

5.1 .2 .

5.2 .3 .

5.1 .2 . 


\begin{tabular}{|c|c|c|c|}
\hline fakultativ & $\begin{array}{l}2.4 \\
2.6\end{array}$ & $\begin{array}{l}\text { Konnexion (primäre Kombinier- } \\
\text { barkeit }\end{array}$ & 2.1 \\
\hline $\begin{array}{l}\text { Fragesatz, indirekter (FRAG) } \\
\left(\begin{array}{l}\text { ob- oder } w \text {-Satz ) }\end{array}\right.\end{array}$ & 6.4 .5 & Konstituenz & $\begin{array}{l}2.1 \\
2.3\end{array}$ \\
\hline $\begin{array}{l}\text { Funktionsverbgefüge } \\
\text { für-Phrase }\end{array}$ & $\begin{array}{l}4.3 .3 . \\
5.4 .2\end{array}$ & Korrelat & $\begin{array}{l}6.3 \cdot \\
6.4 .6 \\
8.4 .\end{array}$ \\
\hline $\begin{array}{l}\text { Genitivergänzung }\left(\mathrm{E}_{2}\right) \\
\text { Gliedsatz }\end{array}$ & $\begin{array}{l}2.5 . \\
5.3 . \\
6.1 .1\end{array}$ & $\begin{array}{l}\text { Korrelat, fakultatives } \\
\text { Korrelat, obligatorisches }\end{array}$ & $\begin{array}{l}6.3 \cdot 3 \\
6.3 \cdot 2 . \\
6.3 \cdot 5\end{array}$ \\
\hline $\begin{array}{l}\text { Hauptsatzförmige Ergänzung } \\
\text { (HPTS) }\end{array}$ & 6.4 .6 & $\begin{array}{l}\text { S. auch SE ohne Korrelat } \\
\text { lexikalische Fügung }\end{array}$ & 4.3. \\
\hline Hilfsverb & 4.6 & $\begin{array}{l}\text { lexikalische Fügung, attri- } \\
\text { buierbare }\end{array}$ & 4.3 .3$. \\
\hline $\begin{array}{l}\text { Hilfsverb vs. Vollverb } \\
\text { Infinitiv }\end{array}$ & $\begin{array}{l}4.6 . \\
4.7\end{array}$ & Lokalbestimmung & $\begin{array}{l}5 \cdot 6 \cdot 2 \\
5 \cdot 6 \cdot 3\end{array}$ \\
\hline Infinitivsatz mit $z u($ INF+) & $\begin{array}{l}5.8 .3 . \\
6.4 .\end{array}$ & $\begin{array}{l}\text { Maschinenausdrucke (Valenz- } \\
\text { lexikon) }\end{array}$ & 9.1. \\
\hline Infinitivsatz ohne $z u$ (INF-) & $\begin{array}{l}5 \cdot 8 \cdot 3 \\
6.4\end{array}$ & $\begin{array}{l}\text { Maschinenausdrucke (Valenz- } \\
\text { register) }\end{array}$ & 9.2 . \\
\hline $\begin{array}{l}\text { Klammer, eckige } \\
\text { Klammer, runde } \\
\text { Klammer, spitze }\end{array}$ & $\begin{array}{l}8.4 . \\
8.4 . \\
8.4\end{array}$ & $\begin{array}{l}\text { mit-Phrase } \\
\text { Modalitätsverb }\end{array}$ & $\begin{array}{l}5.5 \cdot 4 \\
4.7 . \\
4.8 \cdot 4 \\
4.8 .7\end{array}$ \\
\hline $\begin{array}{l}\text { Kommutierbarkeit (siehe Aus- } \\
\text { tauschbarkeit) }\end{array}$ & & $\begin{array}{l}\text { Modalitätśverb vs. Vollverb } \\
\text { Modalverb }\end{array}$ & $\begin{array}{l}4.8 \cdot 4 . \\
4.6 . \\
4.8 \cdot 3\end{array}$ \\
\hline
\end{tabular}




\begin{tabular}{|c|c|c|c|}
\hline Nominativergänzung $\left(E_{0}\right)$ & $\begin{array}{l}2 \cdot 5 \\
5.1\end{array}$ & Präposition & $\begin{array}{l}4.1 . \\
5.5 \cdot 1\end{array}$ \\
\hline $\begin{array}{l}\text { notwendig } \\
\text { ob-Satz s. Fragesatz, indirekter }\end{array}$ & 2.6 & - & $\begin{array}{l}5 \cdot 5 \cdot 2 \\
5 \cdot 5 \cdot 3 \\
5 \cdot 7 \cdot 2 \\
6.3 \cdot 1\end{array}$ \\
\hline $\begin{array}{l}\text { Objekt, inneres } \\
\text { obligatorisch }\end{array}$ & $\begin{array}{l}5.2 .2 \\
2.4 \\
2.6\end{array}$ & $\begin{array}{l}\text { Präpositionalergänzung } \\
\text { (Präpositivergänzung) }\left(\mathrm{E}_{4}\right)\end{array}$ & $\begin{array}{l}2.5 \\
5.5\end{array}$ \\
\hline Paradigma & $\begin{array}{l}2 \cdot 5 \\
5 \cdot 0 \\
5.1 \cdot 2\end{array}$ & $\begin{array}{l}\text { reflexive Fügung } \\
\text { Reflexivpronomen }\end{array}$ & $\begin{array}{l}4.4 .5 \\
4.4 .2 . \\
4.4 .3\end{array}$ \\
\hline $\begin{array}{l}\text { Passivfähigkeit } \\
\text { Passivformen }\end{array}$ & $\begin{array}{l}5.2 \cdot 1 \\
5.2 \cdot 2 \\
7 . \\
7.1\end{array}$ & & $\begin{array}{l}4.4 \cdot 4 . \\
4.4 \cdot 5 \\
4.4 \cdot 6 \\
4.4 .7\end{array}$ \\
\hline Passiv, unpersönliches & $\begin{array}{l}7.3 . \\
7.4\end{array}$ & $\begin{array}{l}\text { Reflexivverb } \\
\text { Regens }\end{array}$ & $\begin{array}{l}4.4 . \\
2.1\end{array}$ \\
\hline Passiv, volles (persönliches) & $\begin{array}{l}7.2 . \\
7.3\end{array}$ & Rektion & 2.2. \\
\hline Pertinenzakkusativ & 5.2 .3 & Satzbauplan (SBP) & 2.6 \\
\hline Pertinenzdativ & $\begin{array}{l}5.2 \cdot 3 . \\
5.4 \cdot 4\end{array}$ & $\begin{array}{l}\text { Satzförmige Ergänzung (SE) } \\
\text { Satzglied }\end{array}$ & $\begin{array}{l}6 . \\
2.4 .\end{array}$ \\
\hline Pertinenzelement & $\begin{array}{l}5.2 .3 . \\
5.4 .\end{array}$ & $\begin{array}{l}\text { Satzmuster (SM) } \\
\text { SE ohne Korrelat. }\end{array}$ & $\begin{array}{l}2.6 . \\
6.3 .4 .\end{array}$ \\
\hline Prädikat, komplexes & $\begin{array}{l}3.2 \cdot 1 \\
4.8\end{array}$ & SE-Form & 6.4 \\
\hline Präfixverben & 4.2 & SE, infinitivische & $\begin{array}{l}4.7 . \\
6.4 .2 . \\
6.4 .3\end{array}$ \\
\hline
\end{tabular}




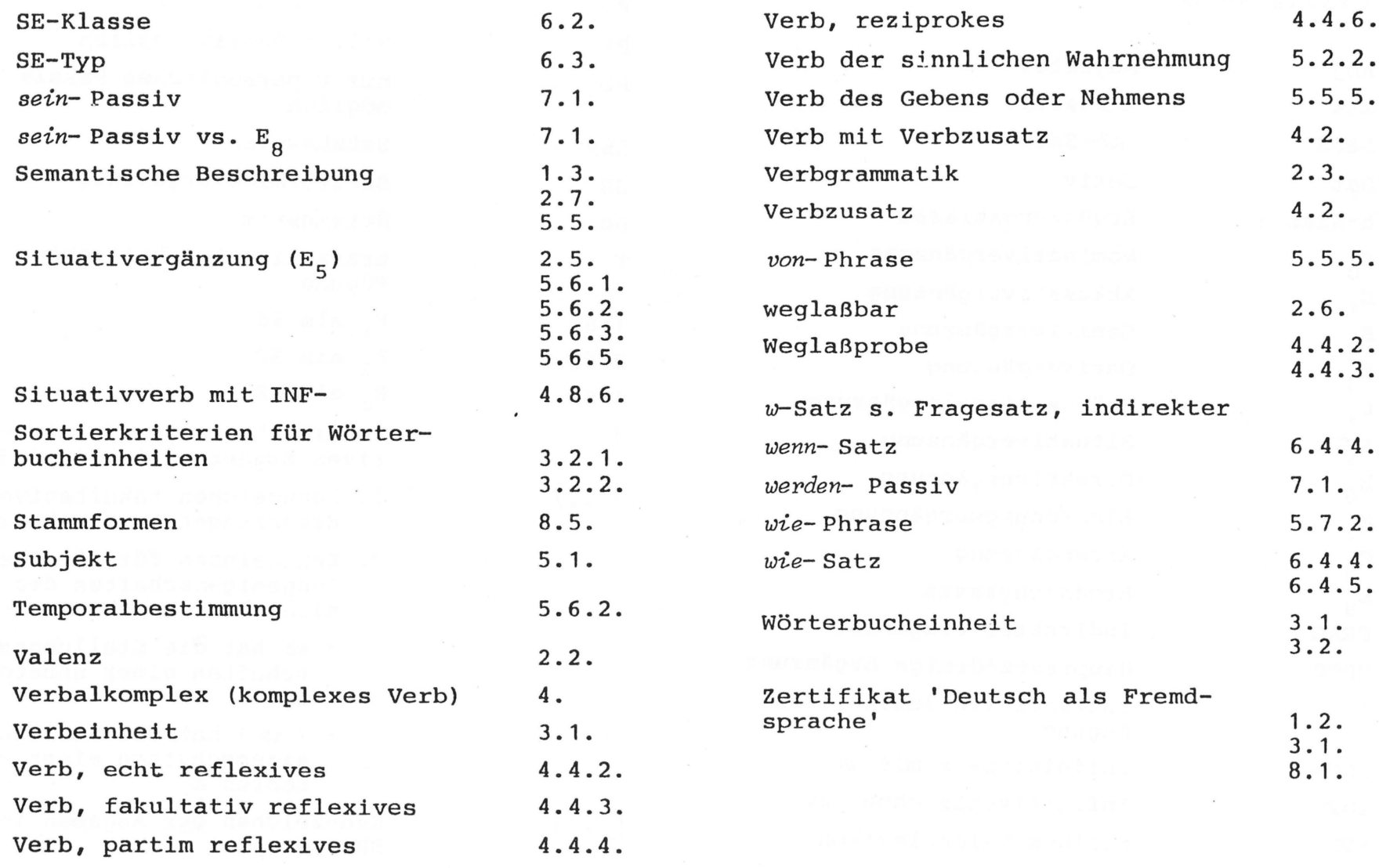




\section{Abkürzungsverzeichnis}

Adj
Akk
DASS
Dat
$E_{-K l a s s e}$
$E_{0}$
$E_{1}$
$E_{2}$
$E_{3}$
$E_{4}$
$E_{5}$
$E_{6}$
$E_{7}$
$E_{8}$
$E_{9}$
FRAG
HPTS
I
INF+
INF-
KVL

Adjektiv

Akkusativ

daß- Satz

Dativ

Ergänzungsklasse

Nominativergänzung

Akkusativergänzung

Genitivergänzung

Dativergänzung

Präpositionalergänzung

Situativergänzung

Direktivergänzung

Einordnungsergänzung

Artergänzung

Ergänzungssatz

indirekter Fragesatz

Hauptsatzförmige Ergänzung

intransitive lexikalische Fügung

Infinitivsatz mit $z u$

Infinitivsatz ohne $z \dot{u}$

Kleines Valenzlexikon
SBP

SE

$S M$

T

$1: S E$

$2: \mathrm{SE}$

$4: S E$

(

$(\ldots)$

[... $]$ kein Passiv möglich

volles Passiv möglich

nur unpersönliches Passiv möglich

Satzbauplan

Satzförmige Ergänzung

Satzmuster

transitive lexikalische Fügung

$E_{1}$ als SE

$E_{2}$ als $S E$

$\mathrm{E}_{4}$ als $\mathrm{SE}$

Kennzeichen einer fakultativen Ergänzung im SBP-Code

1. Kennzeichen fakultativer Ergänzungen im SBP-Beispiel

2. Kennzeichen für die Ste1lungseigenschaften des nicht-kommutierbaren es:

- es hat die Stellungseigenschaften einer unbetonten $\mathrm{E}_{1}$

- (es ) hat die Stellungseigenschaften einer unbetonten $\mathrm{E}_{\mathrm{O}}$

Kennzeichen der Angaben im SBP-Beispiel 
1. Kennzeichen fakultativer Reflexivpronomina in Verbalkomplex und SBP-Beispiel

2. Kennzeichen fakultativer Korrelate $1 \mathrm{~m} \mathrm{SE-}$ Beispiel

1. Codierung des ergänzungslosen SBP

2. Nach Präposition: Kennzeichen des Rektionsverhältnisses

1. Kennzeichen alternativer Besetzung

2. Nach Reflexivpronomen: Kennzeichen dessen nachfolgender Kasusbestimmung

Kennzeichen disjunkter EKlassen im SBP-Code

1. Hochgestellt: Kennzeichen ungrammatischer bzw. inakzeptabler Sätze

2. Nicht hochgestellt: Kennzeichen lexikalischer Fügungen

Kennzeichen der möglichen Aktualisierung einer $\mathrm{E}$ als SE 



\author{
WÖRTERB UCH
}





\section{abbiegen}

abbiegen

BEMERKUNG

\section{abbiegen}

abbiegen

BEMERKUNG :

\section{abfahren}

abfahren

Pertinenzelement im Dativ BEMERKUNG :

\section{abfahren}

abfahren

BEMERKUNG :

\section{abgeben}

abgeben

abgeben

01

abgeben

0113

abgeben ( $a n-A k k)$

$01(4$

abgeben (für-Akk)
P1 Er konnte die Gefahr [gerade noch] abbiegen. STAMMFORMEN: abbiegen, bog ab, hat abgebogen.

P2 Der Autofahrer biegt (nach Zinks) ab. STAMMFORMEN: abbiegen, bog $a b$, ist abgebogen.

P1 Der Autofahrer hat die Reifen [ziemlich schnelZ] abgefahren.

Der Autofahrer fährt dem ilann ein Bein $a b$.

STAMMFORMEN: abfahren, fuhr $a b$, hat abgefahren.

PO Der Zug fährt (von Frankfurt) $a b$.

STAMMFORMEN: abfahren, fuhr $a b$, ist abgefahren.

PO Wolfgang gibt einen guten Ehemann $a b$.

P1 Die Einwohner gaben [bei der Wahl] ihre Stimme ab.

P1 Er gibt (mir) ein Stück $a b$.

P1 Herr Meier gibt sein Amt (an Herrn Schmitz) $a b$.

P1 Er gibt seine Stimme (für den linken Politiker) ab. 


\section{abgeben}

abgeben (gegen - Akk)

$01(4$

abgeben von - Dat

O (34

sich/Akk abgeben mit-Dat

04

4:SE mit obligatorischem Korrelat

\section{abhängen}

abhängen

abhängen

BEMERKUNG :

\section{abhängen}

abhängen von-Dat

04
FRAG :

DASS :

FRAG :
P1 Fr gibt seine Stimme (gegen den Antrag) $a b$.

P2 Er gibt (mir) von seinem Kuchen $a b$.

PO Erma gibt sich [germ] mit Kindern $a b$. Stella gibt sich nicht damit $a b$, auf jeäen kleinsten Fehler hinzuweisen.

P1 Die Diebe hatten die Polizei [nach kurzer Zeit] abgehängt.

P1 Die Mutter hängt [gerade] die Wäsche (von der Leine)ab. STAMMFORMEN : abhängen, hängte $a b$, hat abgehängt.

Po Die Wahl des Berufes hängt von meinen Interessen ab. Viele Studenten hängen [finanziell] von ihren Eltern $a b$.

Es hängt von der Erlaubnis meines Arztes $a b$, ob ich nach Berlin fahren kann.

Das Leben des Verletzten hängt davon $a b$, daß der Krankenwagen rechtzeitig kommt.

Das Leben des Verletzten hängt davon $a b$, ob der Krankenwagen rechtzeitig kommt.

STAMMFORMEN : abhängen, hing ab, hat abgehangen. 
abholen

abholen

$01(6$

ablehnen

ablehnen

1:SE Zum Korrelat vgl. Einleitung 6.3.5.

\section{abmachen}

abmachen mit-Dat

$1: \mathrm{SE}$ mit fakultativem Korrelat

INF+:

DASS :

FRAG :

HPTS :

abmachen

$01(6$

abmelden

abmelden (von-Dat)

INF+ :

DASS :
P1 Der Mann holt das Gepäck (vom Bahnhof) ab.

P1 Der Kronke Zehnt jedes Essen $a b$. Sie lehnt es $a b$, von ihm unterstutzt zu werden. Sie lehnt es $a b$, daß er ihnen hilft.

P1 Ich mache einen Termin mit meinem Arat $a b$. Ich habe <es> mit meiner Frau abgemacht, morgen die Kinder zu hüten.

Ich habe <es > mit meiner Frau abgemacht, daß wir abwechselnd die Kinder hüten.

Ich habe <es> mit meiner Frau abgemacht, wann wir die Kinder hüten.

Ich habe <es > mit meiner Frau abgemacht, ich hüte die Kinder morgen.

P1 Sie macht das Preisschild (von der Schallplatte) ab.

P1 Die Mutter meldet ihren Sohn (von der Schule) ab. Die Angestellte meldet sich (vom Kursus) $a b$. 


\section{abnehmen}

abnehmen

01

\section{Pertinenzelement im Dativ}

abnehmen

$0(1$

abnehmen

013

\section{1:SE mit fakultativem} Korrelat

abnehmen (vor - Dat)

abnehmen

$01(4$

abnehmen (an-Dat)

$01(6$

O 14

abnehmen (um-Akk)

$0(4$

\section{abschleppen}

abschleppen

sïch/Akk abschleppen (mit-Dat) O ( 4

\section{abschließen}

abschließen

abschließen
P1 Die Baupolizei nimmt den Neubau $a b$.

Der Lehrer nimmt die Prüfung $a b$.

Der Chirurg nimmt dem Patienten ein Bein $a b$.

Po Die Frau hat (drei Pfund) abgenommen.

P1 Die Sekretärin nimmt dem Chef die Arbeit ab. Ich nehme ihm diese Geschichte [nicht] $a b$.

DASS :

Ich nehme <es> ihm nicht $a b$, daß er mir wirklich helfen wollte.

P1 Er nahm den Hut (vor ihm) ab.

P1 Er nimnt das Bizd (von der Wand) $a b$.

PO Die Sonnenstrahlen nehmen [gegen Abend] [schne II] (an Intensität) $a b$.

po Der Mond nimmt (um ein Viertel) $a b$.

P1 Die Polizei schleppte das Auto (von der Straße) ab.

PO Ich schleppe mich (mit den Paketen) $a b$.

P1 Wir haben einen Vertrag [mit dem Hausbesitzer] abgeschlossen.

P1 Mutter schließt (die Tür) $a b$. 
abschließen

abschließen mit - Dat

abschließen

\section{abschmieren}

abschmieren

\section{abtrocknen}

abtrocknen

\section{Pertinenzelement im Dativ} BEMERKUNG :

\section{abtrocknen}

abtrocknen

BEMERKUNG :

\section{abwarten}

abwarten

1:SE ohne Korrelat
$01(8$

abgeschlossen.

Po [In diesem Roman] schließt der Dichter mit der Welt $a b$.

PO Das Geschäftsjahr schloß mit Erfolg $a b$.

P1 Der Mechaniker schmiert den Wagen $a b$.

P1 Die Mutter trocknet (das Geschirr) $a b$. Das Kind trocknet sich $a b$.

Die Mutter trocknet dem Kind das Gesicht ab. STAMMFORMEN: abtrocknen, trocknete $a b$, hat abgetrocknet.

Po [Nach dem Gewitter] ist die Straße [schnell] abgetrocknet.

STAMMFORMEN : abtrocknen, trocknete $a b$, ist abgetrocknet.

P1 Das Mädchen wartet die letzte Straßenbahn ab. FRAG: Wir warten $a b$, ob das Wetter besser wird. 


\section{achten}

achten

achten auf - Akk

4:SE mit obligatorischem Korrelat

\section{anbieten \\ anbieten \\ 1:SE mit fakultativem Korrelat}

0113

\section{ändern}

ändern (an - Dat)

$01(4$

O:SE Zum Korrelat vgl.

4:SE mit obligatorischem Korrelat
INF+:

DASS :

01

04

INF+:

DASS :

FRAG :

DASS:

\section{DASS :}

DASS :

FRAG :
P1 Wir achten seine Entscheidung.

P2 Sie achtet ouf ihre Gesundheit.

Sie achtet darauf, immer püntlich zu sein.

Sie achtet darouf, daß die Blumen genug Wasser bekommen.

Sie hat nicht darauf geachtet, ob das Auto rot oder blau gewesen ist.

P1 Der Makler bietet (dem Mann) eine Wohnung an.

Der kleine Junge bietet 〈es〉 dem Mann an, ihn über die Straße zu führen.

Meine Freundin hat 〈es> mir angeboten, daß ich am Wochenende ihr Auto benützen könnte.

P1 Das neue Gesetz ändert wenig (an unserer Situation). Es ändert nichts an unserer Laune, daß wir jetzt eine andere Arbeit machen müssen.

Sein Kommen ändert nichts daran, daß ich schlechte Laune habe.

Seine Stimme ändert nichts daran, wie die Wahl ausgeht. 


\begin{tabular}{|c|c|c|c|c|}
\hline BEMERKUNG: & & & & $\mathrm{E}_{1}$ meist indefinit. \\
\hline sich/Akk ändern & 0 & . & PO & Die Zeiten änderm sich. \\
\hline sich/Akk ändern (an-Dat) & $0(4$ & & Po & $\begin{array}{l}\text { Es hat sich [noch] nichts (an meiner Situation) } \\
\text { geändert. }\end{array}$ \\
\hline $\begin{array}{l}\text { 4:SE mit obligatorischem } \\
\text { Korrelat }\end{array}$ & & DASS : & & $\begin{array}{l}\text { Bis jetzt hat sich nichts daran geändert, daß er } \\
\text { immer zu spät kommt. }\end{array}$ \\
\hline BEMERKUNG : & & & & $\mathrm{E}_{\mathrm{o}}$ meist indefinit. \\
\hline anfahren & & & & \\
\hline anfahren & 01 & & P1 & Der Autofahrer hat ein Kind angefahren. \\
\hline $\begin{array}{l}\text { BEMERKUNG : } \\
\text { anfahren }\end{array}$ & & & & STAMMFORMEN : anfahren, fuhr an, hat angefahren. \\
\hline anfahren & 0 & & PO & Der Wagen fuhr [Zeise] an. \\
\hline $\begin{array}{l}\text { BEMERKUNG: } \\
\text { anfangen }\end{array}$ & & & & STAMMFORMEN: anfahren, fuhr an, ist angefahren. \\
\hline anfangen & 01 & & P1 & Die Hausfrau fängt ihre Arbeit an. \\
\hline 1.SE ohne Korrelat & & INF+: & & Die Hausfrau fängt zu arbeiten an. \\
\hline änfaingen & $0(17$ & & PO & $\begin{array}{l}\text { Der Millionär hat (seine Laufbahn) als Teller- } \\
\text { wäscher angefangen. }\end{array}$ \\
\hline anfangen & $01(8$ & & P1 & Wir fangen die Sache (geschickt) an. \\
\hline anfangen mit-Dat. & 04 & & $\mathrm{P} 2$ & Die Hausfrau fängt mit dem Abtrocknen an. \\
\hline
\end{tabular}




\begin{tabular}{|c|c|c|c|c|c|}
\hline \multicolumn{2}{|c|}{ 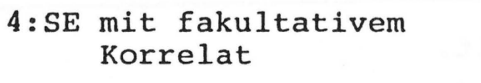 } & \multicolumn{3}{|c|}{ INF+: } & $\begin{array}{l}\text { Die Hausfrau fängt <damit>an, das Geschirr abzu- } \\
\text { trocknen. }\end{array}$ \\
\hline \multicolumn{2}{|c|}{$\begin{array}{l}4: \text { SE mit obligatorischem } \\
\text { Korrelat }\end{array}$} & & DASS : & & $\begin{array}{l}\text { Die Sitzung fängt domit an, daß die Tagesordnung } \\
\text { verlesen wird. }\end{array}$ \\
\hline \multicolumn{2}{|c|}{ anfangen } & $O(5$ & & Po & Die Vorstellung fängt (in einer halben Stunde) an. \\
\hline \multicolumn{6}{|c|}{ anfassen } \\
\hline \multicolumn{2}{|c|}{ anfassen } & 01 & & P1 & Der kleine Junge faßt die Katze [nicht gern] an. \\
\hline \multicolumn{2}{|c|}{ anfassen } & 018 & & P 1 & Der Lehrer faßt die Schüler hart an. \\
\hline \multicolumn{6}{|c|}{ angehen } \\
\hline \multicolumn{2}{|c|}{ angehen } & 01 & & P1 & Der Hund geht den Briefträger an. \\
\hline \multicolumn{2}{|c|}{ BEMERKUNG : } & & & & STAMMFORMEN: angehen, ging an, hat angegangen. \\
\hline \multicolumn{2}{|c|}{ angehen } & & & & \\
\hline \multirow{2}{*}{\multicolumn{2}{|c|}{ angehen }} & 0 & & PO & Das Licht geht an. \\
\hline & & & & & Die Pflanzen sind angegangen. \\
\hline \multicolumn{2}{|c|}{ angehen } & 011 & & PO & Meine Arbeit geht dich nichts an. \\
\hline \multirow[t]{2}{*}{$\mathrm{O}: \mathrm{SE}$} & $\begin{array}{l}\text { Zum Korrelat vgl. } \\
\text { Einleitung } 6.3 .5\end{array}$ & & DASS : & & $\begin{array}{l}\text { Es geht niemanden etwas an, daß Frau Miiller ge- } \\
\text { schieden ist. }\end{array}$ \\
\hline & & & FRAG : & & Es geht dich nichts an, was in diesem Brief steht. \\
\hline \multicolumn{2}{|c|}{$\begin{array}{l}\text { BEMERKUNG : } \\
\text { angehen um - Akk }\end{array}$} & 014 & & P1 & $\begin{array}{l}\text { Unpersönliche } \mathrm{E}_{1} \text { meist indefinit. } \\
\text { Der Sohn ging den Vater um Geld an. }\end{array}$ \\
\hline
\end{tabular}




\section{angehen}

018

angehen gegen - Akk

04

angehen

$O(5$

BEMERKUNG :

\section{ankommen}

ankommen

018

O:SE Zum Korrelat vgl. Einleitung 6.3.5.

ankommen gegen - Akk

04

ankommen

$0(5$

ankommen (bei- Dat)

$0(4) 8$

ankommen (es) auf-Akk

ankommen (es) auf-Akk (bei-Dat) 4 (4

4:SE mit obligatorischem Korrelat

INF+:

DASS :

FRAG :

anmachen

anmachen
P1 Er geht das Problem geschickt an.

P2 Der Rechtsanwalt geht gegen die Entscheidung des Gerichts an.

Po Das Theater geht (um $20 \mathrm{Uhr}$ ) an. STAMMFORMEN: angehen, ging an, ist angegangen.

PO Dieser Verzicht kommt i.hn schwer an.

INF+: Es kam mich schwer an, auf die Reise zu verzichten.

DASS: $\quad$ Es kam mich schwer an, daß ich auf die Reise verzichten mußte.

Po Gegen ihre Uberredungskünste kam niemand an.

Po Der Zug kam (in Heidelberg) an.

PO Seine Idee kommt (gut) (beim Chef) an.

PO Es kommt (ihm) auf fünf Mark [nicht] an.

PO (Bei diesem Test) kommt es [vor allem] auf schnelle Reaktion an.

Es kommt darauf an, schnelz zu sein.

Es kommt darauf an, daß er schnell ist.

Es kommt darauf an, wie schnelz er ist.

P1 Er macht das Licht an. 
anmachen

$01(8$

$01(5$

anmelden

conmelden

01

01

annehmen

1:SE ohne Korrelat

1:SE mit fakultativem Korrelat

\section{BEMERKUNG :}

annehmen (von-Dat)

$01(4$

annehmen

017

sich/Akk annehmen

02

01

018

01

$0(1$
P1 Die Mutter macht den Salat (mit or) an.

P1 Die Mutter meldet ihr Kind (beim Arzt) an. Die Mutter meldet sich (beim Arzt) an.

P1 Meine Wirtin hat das Paket [nicht] angenommen.

P1 Ich nehme die Wahrheit dieses Satzes [einmal] an.

HPTS: Ich nehme an, du hast nach der langen Reise Hunger.

DASS: $\quad$ Ich nehme <es $>$ an, daß dieser Satz richtig ist.

$E_{1}$ meist indefinit oder SE.

P1 Er nahm das Geschenk (von seinen Freunden) an.

P1 Er nahm ihn als Sohn an.

Po Der Vormund nimmt sich des Kindes an.

P1 Er redet ihn [auf der Straße] an.

P1 Er redet ihn mit Sie an.

P1 Der Posten ruft den Soldaten an.

P1 Der Ehemann ruft (seine Frau) an. 
anrufen (um-Akk)

$01(4$

4:SE ohne Korrelat

4:SE mit obligatorischem Korrelat

anrufen

ansehen

ansehen

ansehen

1:SE mit fakultativem Korrelat

ansehen für - Akk/Adj

\section{1:SE Zum Korrelat vgl.} Einleitung 6.3.5.

ansehen

1:SE Zum Korrelat vgl. Einleitung 6.3.5.
P1 [In der Not] mufen wir Gott (um Hilfe) an.

Mon ruft das Bundesverfassungsgericht an, die Verfassungsmäßigkeit eines Gesetzes zu prüfen.

DASS : Sie rief Gott damu an, daß er ihr vergebe.

P2 Der Ehemann ruft (zu Hause) an.

P1 Er sah seine Freundin [verstohlen] an.

P1 Man sieht diesem Mann seine Müdigkeit an.

DASS :

Man sieht <es> dem Mann an, daß er müde ist.

FRAG : Man sieht <es> dem Mann nicht an, ob er müde ist.

HPTS : Man sieht <es> ihm an, er ist müde.

P1 Der Polizist sah den Jungen für den Dieb an. Wir sehen die Entscheidung für falsch an.

INF+:

DASS : Ich sehe es für einen Fehler an, seinen Vorschlag abzulehnen.

Wir sehen es für falsch an, daß das Projekt $a b-$ gebrochen wurde.

P1 Er sieht den Lottogewinn als ein Geschenk. des Hinmels an.

INF+: Ich sehe es als Arbeit an, Briefe zu schreiben. 
1:SE Zum Korrelat vgl. Einleitung 6.3.5.

ansehen

<sich/Dat> ansehen

$1: \mathrm{SE}$ Zum Korrelat vgl. Einleitung 6.3 .5 .

\section{antworten}

antworten (auf-Akk)

$1: \mathrm{SE}$ ohne Korrelat

$011(3) 4$

DASS :

HPTS :

BEMERKUNG :

\section{anzeigen}

anzeigen

01

1:SE ohne Korrelat

anzeigen (bei-Dat)

DASS :

FRAG:

018

01

FRAG:

DASS :

FRAG :

$01(4$
P1 Hans sieht den Fall anders an als Karl.

PO Der Arzt sah <sich> die Verletzung an.

Der Arzt sieht sich an, wie schwer der Mann verletat ist.

P1 Er antwortet (mir) (nichts) (auf meine Frage). Auf unsere Fragen antwortete er, daß er kein Geld hätte.

Auf unsere Fragen antwortete er schließlich, er hätte kein Geld.

$E_{1}$ meist indefinit oder SE.

P1 Der Radfahrer zeigt die Richtung an.

Ich sehe es als Fehler an, daß diese Stellung ausgerechnet ihm angeboten wurde.

Ich sehe es als entscheidend an, ob du gelogen hast oder nicht.

Der Radfahrer zeigt an, daß er die Richtung änderm wilz.

Der Radfahrer zeigt an, wohin ex fahren wizl.

P1 Der Fußgänger zeigt den Autofahrer (bei der Polizeil on. 


\section{anziehen}

anziehen

anziehen

anziehen

anziehen

\section{anzünden}

anzünden

Pertinenzelement im Dativ

\section{arbeiten}

arbeiten

arbeiten (an - Dat)

$4:$ SE mit obligatorischem Korrelat

arbeiten (für-Akk)

arbeiten

O $(4$
PO Die Preise ziehen an.

P1 Emil zieht die Bremse an.

Ihre schönen Beine ziehen die Blicke vieler Männer an.

Die Bäume ziehen den Blitz an.

P1 Die Mutter zieht dem Kind die Schuhe an. Die Mutter zieht sîch die Schuhe an.

P1 Claudia zieht ihre Söhne [immer] (gut) an. Ich ziehe mich [immer] (gut) an.

P1 Michael zündet ein Streichholz an. [Am Lagerfeuer] hat sie sich [aus Leichtsinn] die Haare angezündet.

PO Die neue Maschine arbeitet [hervorragend].

P2 Der Mechaniker arbeitet (an einem Auto).

INF+: Di.e Firma arbeitet daran, das Produkt zu verbessern.

P2 Hans arbeitet (für einen Zeitschriftenveriag).

O (5 P2 Ich arbeite (bei einer großen Firma). 
arbeiten

07

ärgern

ärgern

ärgern

O:SE Zum Korrelat vgl. Einleitung 6.3.5.

sich/Akk ärgern (über-Akk)

4:SE mit obligatorischem Korrelat

\section{atmen}

atmen

atmen

atmen

\section{aufgeben}

aufgeben

aufgeben

1:SE Zum Korrelat vgl. Einleitung 6.3.5.
01

01

INF+:

DASS :

$014(8$

INF+:

DASS :

0

01

08

01
Po Mein Vater arbeitet als Fahrer.

P1 Ich ärgere meinen Vater.

PO Deine Tat ärgert mich. Es ärgert mich, dich hier zu finden.

Es ärgert mich, daß du nicht gekommen bist.

PO Er ärgert sich (krank) (über ihre Antwort). Er ärgert sich dariber, nicht gefragt zu werden. Er ärgert sich darüber, daß er nicht gefragt wurde.

po Die Haut atmet.

PO Dieser Ort atmet Ruhe.

P2 Der Kranke atmet unregelmäßig.

P1 Die Sekretärin gibt ein Telegramm auf. Der Vater gibt das Geschäft auf. Der Arzt gibt den Kranken auf.

P1 Der Kranke gibt die Hoffnung auf Besserung auf. INF+: $\quad$ Der Kranke hat es aufgegeben, auf Besserung zu hoffen. 
aufgeben

aufgeben

$1: \mathrm{SE}$ ohne Rorrelat

$O(1$

$01(3$

\section{INF+:}

\section{aufheben}

oufheben

aufheben (für - Akk)

$01(4$

oufheben

$01(6$

\section{aufhören}

aufhören

aufhoren mit-Dat

4:SE ohne Korrelat

\section{aufmachen}

aufmachen

Pertinenzelement im Dativ

oufmachen
P1 Der Sportler gibt (das Rennen) auf.

P1 Der Lehrer gibt (den Schülerm) ein Zanges Gedicht auf. Der Lehrer gibt den Schülern auf, das Gedicht auswendig zu Zemen.

P1 Die neue Regierung hebt das Gesetz auf.

Die Gastgeberin hebt die Tafel auf.

P1. Die Elterm heben das Geld (für ihre Kinder) auf.

P1 Fritzchen hebt das Buch (vom Boden) ouf.

PO Die Straße hört [dort hinten] auf.

P2 Hört endlich mit dem Geschrei auf!

INF+: Fritzchen hört auf zu Zernen.

P1 Er macht die Tür auf.

Er macht einen Laden auf.

Der Handwerker macht seine Rechnung auf.

Er macht ihr das KZeid auf.

P1 Der Verlag hat das Buch ansprechend aufgemacht.

P2 Die Kaufhäuser machen (um neun Uhr) auf. 
sich/Akk aufmachen

$O(6$

01

aufnehmen

018

aufnehmen

1:SE Zum Korrelat vgl. Einleitung 6.3.5.

aufnehmen es mit-Dat

04

\section{aufpassen}

aufpassen

013

aufpassen (auf-Akk)

$0(4$

\section{4:SE mit fakultativem} Korrelat

\section{aufräumen}

aufräumen

aufräumen (mit-Dat)

$0(4$

aufräumen unter-Dat po Ich mache mich (nach Hause) auf.

P1 Sie nahmen den Fremden [freundlich] ouf.

Der Kaufmann nimmt [bei der Bank] einen Kredit auf.

P1 Die Opposition hat den Plan der Regierung positiv aufgenommen.

DASS : Die Bevölkerung hat es positiv aufgenommen, daß die Regiemung die Renten erhöhen will.

PO Er nimmt es mit jedem auf.

P1 Die Verkäuferin paßt der Kundin den Hut auf.

P2 Der Hund paßt (auf die Kinder) auf. Die Jungen passen (auf die Worte des Lehrers) auf.

DASS: $\quad$ Er paßt <darauf > auf, daß er nicht zu dick wird. FRAG: $\quad$ Er paßt <darauf> auf, ob die Maschine richtig arbeitet.

P1 Das Mädchen räumt (das Zinmer) auf.

P2 Der neue Abteilungsleiter räumt (mit der Schlamperei) [gründiich] auf.

P2 Die Seuche röumte unter der Bevölkerung auf. 


\section{aufregen}

aufregen

01

O:SE Zum Korrelat vgl. Einle1tung 6.3 .5

sich/Akk aufregen (über-Akk)

4:SE mit obligatorischem Korrelat

\section{aufschreiben}

oufschreiben

01

\section{1:SE mit fakultativem Korrelat}

\section{aufstehen}

aufstehen

0

BEMERKUNG :

\section{aufstehen}

oufstehen

oufstehen

aufstehen
P1 Der Lärm regt mich auf.

INF+: Es regt mich auf, lange warten zu müssen.

DASS: $\quad$ Es regt mich auf, daß er nicht kommt.

Po Er regt sich (über ihre Antwort) auf.

INF+: Der Vater regt sich darüber auf, immer kritisiert zu werden.

DASS: $\quad$ Ich rege mich daruber auf, daß er nicht gekommen ist.

P1 Der Polizist schreibi das falsch parkende Auto auf.

DASS: Der Lehrer schreibt <es> auf, daß Hans zu spät gekommen ist.

FRAG :

Der Schüler schreibt <es> auf, was er gesehen hat.

PO Die Tür steht auf.

STAMMFORMEN: steht auf, stand auf, hat aufgestanden.

PO Das Volk steht auf.

PO Ich stehe [meistens] (um sechs) auf.

PO Claudia steht (vom Tisch) auf.

STAMMFORMEN : steht auf, stand auf, ist aufgestanden.

BEMERKUNG : 


\section{aufwachen}

aufwachen (aus-Dat)

$O(4$

ausgeben

ausgeben

ausgeben ( $f u r$ - Akk)

$4: \mathrm{SE}$ mit obligatorischem Korrelat

01

$01(4$

INF+ :

DASS

ausgeben

\section{ausmachen}

ausmachen

ausmachen

O:SE Zum Korrelat vgl. Einleitung 6.3.5.

\section{BEMERKUNG :}

ausmachen (mit-Dat)

1:SE mit fakultativem Korrelat

INF+:

DASS :

FRAG :

$01(4$
PO Erwacht (aus der Ohnmacht) auf.

P1 Er gibt eine Runde aus.

P1 Sie hat (fur diese Geschenke) viel Geld ausgegeben. sie gibt viel Geld dafür aus, andere Leute zu beschenken.

Der Staat gibt viel Geld dafür aus, daß neue Straßen gebaut werden.

P1 Ergibt seinen Freund als Ausländer aus.

P1 Die Kinder machen das Licht aus. Der Soldat macht die Richtung aus.

PO Das macht mir nichts aus. Es macht mir nichts aus, kein Geld zu haben. Es macht mir nichts aus, daß ich kein Geld habe. Es macht mir nichts aus, ob ich Geld habe oder nicht. $\mathrm{E}_{1}$ meist indefinit.

P1 Karl macht (mit seiner Freundin) ein Treffen aus. INF+: Die Freunde machen $\langle e s\rangle$ aus, sich nächste Woche zu treffen. 
1:SE mit fakultativem Korrelat

\section{ausnutzen}

cusnutzen

1:SE Zum Korrelat vgl. Einleitung 6.3.5.

\section{auspacken}

auspacken

auspacken

0

$0<1$

ausrechnen

ausrechnen

$1:$ SE mit fakultativem Korrelat

01

DASS :

FRAG :

01

DASS :

P1 Die Studenten nutzen ihre Vorteile aus.

Die Freunde machen <es > aus, daß sie sich nächste Woche treffen wollen.

Die Freunde machen <es> aus, wo sie sich treffen wollen.

P2 Der Angeklagte packt aus.

P1 Ich packe (den Koffer) aus.

P1 Die Hausfrau rechnet die Kosten der Urlaubsreise aus. DASS: Die Hausfrau rechnet <es> aus, daß die Urlaubsreise zu teuer wird.

FRAG :

<sich/Akk > ausruhen

\section{ausschließen}

ausschließen
01
Die Hausfrau hat <es> ausgerechnet, wieviel Geld sie im Urlaub ausgeben darf.

P2 Der Sportler ruht <sich> aus.

P1 Die Polizei schließt einen Selbstmord [nicht] aus. . 


\begin{tabular}{|c|c|c|c|c|}
\hline $\begin{array}{l}1: \text { SE mit fakultativem } \\
\text { Korrelat. }\end{array}$ & & DASS : & & $\begin{array}{l}\text { Die Polizei schließt <es> nicht völlig aus, daß } \\
\text { hier ein Fall von Selbstmord vorliegt. }\end{array}$ \\
\hline ausschließen (von-Dat) & $01(4$ & & P1 & $\begin{array}{l}\text { Die Wettkampfleitung schloß den Sportler (von der } \\
\text { weiteren Teilnahme) aus. }\end{array}$ \\
\hline $\begin{array}{l}\text { ausschließen } \\
\text { aussehen }\end{array}$ & $01(6$ & & P1 & $\begin{array}{l}\text { Der Vorstand schließt einige Mitglieder (aus dem } \\
\text { Verein) aus. }\end{array}$ \\
\hline aussehen nach-Dat & 04 & & Po & $\begin{array}{l}\text { Das sieht [mir] [ganz] nach Betrug aus. } \\
\text { Dieses Kleid sieht nach nichts aus. }\end{array}$ \\
\hline aussehen & 08 & & Po & $\begin{array}{l}\text { Das Schloß sieht alt aus. } \\
\text { [Fur ihn] sieht die Sache schlecht aus. }\end{array}$ \\
\hline aussehen (es) mit-Dat & 48 & & Po & Mit ihm sieht es schlecht aus. \\
\hline aussprechen & & & & \\
\hline aussprechen & 01 & & P1 & Das Kind spricht einen wunsch aus. \\
\hline aussprechen & 013 & & P1 & Der Chef spricht den Mitarbeiterm seinen Dank aus. \\
\hline sich/Akk aussprechen für-Akk & 04 & & Po & $\begin{array}{l}\text { Der Versammlungsleiter sprach sich für eine Ver- } \\
\text { tagung aus. }\end{array}$ \\
\hline $\begin{array}{l}4: \mathrm{SE} \text { mit obligatorischem } \\
\text { Korrelat }\end{array}$ & & INF+: & & $\begin{array}{l}\text { Der Versammlungsleiter sprach sich dafür aus, die } \\
\text { Sitzung zu vertagen. }\end{array}$ \\
\hline & & DASS : & & $\begin{array}{l}\text { Der Versanmlungsleiter sprach sich dafür aus, daß } \\
\text { die Sitzung vertagt werden solle. }\end{array}$ \\
\hline sich/Akk aussprechen gegen-Akk & 04 & & Po & $\begin{array}{l}\text { Die Opposition sprach sich gegen den neuen Gesetz- } \\
\text { entwurf aus. }\end{array}$ \\
\hline $\begin{array}{l}\text { 4:SE mit obligatorischem } \\
\text { Korrelat }\end{array}$ & & INF+: & & $\begin{array}{l}\text { Die Opposition sprach sich dagegen aus, den Vertrag } \\
\text { zu ratifizieren. }\end{array}$ \\
\hline
\end{tabular}


4:SE mit obligatorischem Korrelat

sich/Akk aussprechen (über-Akk) O(4

\section{aussteigen}

aussteigen

ausstellen

ausstellen

ausstezien

013

\section{aussuchen}

aussuchen

auswandern

01

\section{ausziehen}

ausziehen

ausziehen

BEMERKUNG :

\section{ausziehen}

ausziehen
DASS: $\quad$ Die Opposition sprach sich dagegen aus, daß der Vertrag ratifiziert wird.

PO Das Ehepaar spricht sich (über seine Probleme) aus.

P2 Er steigt (aus dem Auto) aus.

Er steigt (aus dem Geschäft) aus.

P1 Der Maler stezlt seine Bilder aus.

P1 Der Chef stellt dem Arbeiter ein gutes Zeugnis aus.

P1 Birgit sucht einen neuen Mantel aus.

P2 Vieie Europäer wandern (nach Australien) aus.

P1 Emiz zieht den Tisch aus.

P1 Die lutter zieht dem Kind den Pullover aus. STAMMFORMEN : zieht aus, zog aus, hat ausgezogen.

P2 Der Mieter zieht (aus der Wohnung) aus. STAMMFORMEN : zieht aus, zog aus, ist.ausgezogen. 


\section{backen}

backen

$0(1$

baden

baden

<sich/Akk > baden

01

0

bauen

bauen

01

bauen

0116

bauen auf-Akk

04

4:SE mit obligatorischem Korrelat

\section{beachten}

beachten

1:SE mit fakultativem Korrelat

\section{beantragen}

beantragen

01

01
P1 Unser Sohn hat [heute] (einen. Kuchen) gebacken.

P1 Die Mutter badet das Baby.

PO Die Kinder baden <sich〉.

P1 Wir bauen ein Haus.

P1 Wir bauen unser Haus (an den Berg).

P2 Er baut auf die Loyalität seines Freundes.

INF+: $\quad$ Er baut fest darauf, einen Kredit zu bekommen.

DASS: $\quad$ Er baut darauf, daß sein Freund verschwiegen ist.

P1 Der Kranke beachtet die Ratschläge seines Arztes. DASS: Du hast <es> nicht beachtet, daß die Fahrpläne sich geöndert haben.

P1 Der Arbeiter beantragt Uriaub.

INF+:

DASS :
Der Arbeiter beantragt, Gefahrenzulage zu bekommen.

Der Arbeiter beantragt, daß er Sonderurlaub bekommt. 


\section{bedanken}

sich/Akk bedanken (bei-Dat)

$O 14(4$

( $f u r$ - Akk)

4:SE mit obligatorischem Korrelat

\section{bedeuten}

bedeuten

1:SE ohne Korrelat

BEMERKUNG :

bedeuten

039

9: INF+:

DASS :

HPTS :

\section{bedienen}

bedienen

bedienen

sich/Akk bedienen

\section{beeilen}

sich/Akk beeilen (mit-Dat)
01

DASS :

\section{P2}

PO Er bedankt sich (bei seinen Kollegen) (für das Geschenk).

Er bedankt sich dafür, daß man ihm diese Arbeit abgenommen hat.

PO Geld bedeutet [mir] [nicht] viel. Seine Worte bedeuten, daß ich entlassen bin. $E_{1}$ meist indefinit.

Er bedeutet ihr, den Raum zu verlassen.

Er bedeutet ihr, daß sie den Raum verlassen soll. Er bedeutet ihr, sie solle den Kaum verlassen.

P1 Der Kellner bedient den Gast.

Die Lufthansa bedient die Strecke Frankfurt-Moskau.

P1 Der Skatspieler bedient (Pique).

Po Der Ausländer bedient sich des Wörterbuchs.

PO Der Schüler beeilt sich (mit den Hausaufgaben). 
$4: S E$ mit fakultativem Korrelat

INF+:

\section{begegnen}

begegnen

03

begegnen

\section{beginnen}

beginnen

beginnen

beginnen

018

beginnen mit-Dat

4:SE mit fakultativem Korrelat

4:SE mit obligatorischem Korrelat

beginnen

$0(5$

\section{begrüßen}

begrüßen

1:SE Zum Korrelat vgl. Einleitung 6.3.5.

DASS :

$\mathrm{INF}+$ :
Die Schüler beeilen sich <damit>, ihre Hausaufgaben zu erledigen.

PO Ich bin meinem Nachbarn begegnet.

P2 Wir begegnen dem Vater mit Achtung.

P1 Die Hausfrau beginnt ihre Arbeit.

PO Kaum ein Millionär hat (seine Laufbahn) [tatsächlich] als Tellerwäscher begonnen.

P1 Die Mannschaft begann die zweite Halbzeit offensiv.

P2 Die Hausfrau beginnt mit ihrer Arbeit.

INF+: Die Hausfrau beginnt 〈damit>, das Geschirr abzutrocknen. Die Sitzung beginnt damit, daß die Tagesordnung verZesen wird.

Po Die Theatervorstellung hat (vor einer halben Stunde) begonnen.

P1 Die Housfrau begrüßt die Gäste.

Die Regierung begrüßt den Verhandlungserfolg.
Die Schuler begrüßen es, einen neuen Lehrer zu bekommen. 
1:SE Zum Korrelat vgl.

Einleitung 6.3 .5 .

\author{
behalten \\ behalten \\ behalten \\ 1:SE mit fakultativem \\ Korrelat
}

01

015

DASS :

FRAG :

* für sich behalten

\section{behandeln}

behandeln

behandeln

behandeln

018

\section{behaupten}

behaupten

behaupten
DASS :

Die Regierung begrüßt es, daß die Opposition sie unterstutzen wizl.

P1 Die Firma behält den Angestelzten.

P1 Er behält den Urlaub in guter Erinnerung.

Ich werde <es > immer in Erinnerung behalten, daß du mir geholfen hast.

Ich werde <es > immer in Erinnerung behalten, wie du mir geholfen hast.

PO Er behält das Geheimnis für sich.

P1 Die Schuler behandeln dieses Thema.

Der Arzt behandelt Hans.

P1 Die Mutter behandelt ihren erwachsenen Sohn wie ein Kind.

P1 Wir behandeln ihn schlecht.

P1 Er behouptet seine Stellung.

P1 Er behouptet viel. 


\section{1:SE mit fakultativem} Korrelat

\section{BEMERKUNG :}

sich/Akk behaupten

(gegen-Akk)

\section{bekanntmachen}

bekanntmachen

bekanntmachen mit-Dat

\section{bekommen}

bekommen

bekommen (von - Dat)

$1: \mathrm{SE}$ ohne Korrelat

békonmen

*in seine Gewalt bekommen

*zu tun bekommen es mit-Dat

BEMERKUNG :

\section{bekommen}

bekommen
$O(4$

01

$01(4$

014

INF+:

DASS :

HPTS :

014

018

$\mathrm{T}$

I

$03(8$
Er behauptet 〈es>, mich zu Zieben.

Er behouptet 〈es>, daß er mich liebt.

Er behauptet $\langle e s\rangle$, er liebe mich.

$\mathrm{E}_{1}$ meist indefinit oder $\mathrm{SE}$.

PO Er behauptet sich (gegen die Familie).

P1 Jörg macht (uns) seine Ideen bekannt.

P1 Almut macht ihren Vater mit ihrem Freund bekannt.

PO Die Lehrerin bekam einen Sohn.

Hans bekommt Arbeit.

Po Ich habe das Foto (von meinem Freund) bekommen. Ich habe von ihm zu essen bekommen.

PO Die Mutter bekommt das Geschirr sauber.

Po Die Polizei bekommt den Verbrecher in ihre Gewalt.

PO Er bekommt es mit der Angst zu tun.

STAMMFORMEN : bekommt, bekam, hat bekommen.

PO Das Brot bekommt mix (gut). 
O:SE Zum Korrelat vgl. Einleitung 6.3.5.

\section{BEMERKUNG :}

\section{belegen}

belegen

belegen mit-Dat

belegen (mit-Dat)

$01(4$

\section{beleidigen}

beleidigen

01

\section{bemerken}

bemerken

1:SE mit fakultativem Korrelat

bemerken zu-Dat
INF+:

DASS : Es bekommt mir gut, weniger als früher zu rauchen. Es bekommt mir gut, daß ich wenig esse. STAMMFORMEN : bekommen, bekam, ist bekommen.

P1 Ich belege zwei Plätze.

Der Student belegt die Vorlesung.

P1 Der Richter belegt den Angeklagten mit einer empfindlichen Strafe.

P1 Ich belege den Kuchen (mit obst).

P1 Karl beleidigt seinen Freund.

P1 Der Dieb bemerkt den Hund.

DASS : Der Dieb bemerkt 〈es> zu spät, daß ein Hund im Hause ist.

FRAG : Wir haben <es> nicht bemerkt, wie der Dieb ins Haus kam.

P1 Er bemerkte etwas zu diesem Problem.

DASS: $\quad$ Er bemerkte zu diesem Problem, daß es so nicht zu Zösen sei. 


\section{$1:$ SE ohne Korrelat}

HPTS :

BEMERKUNG :

\author{
bemühen \\ bemühen \\ sich/Akk bemühen (um-Akk) \\ $4: S E$ mit fakultativem \\ Korrelat
}

$01(6$

$\mathrm{O}(4$

INF+

DASS :

\section{beobachten}

beobachten

1:SE mit fakultativem Korrelat

01

DASS :

FRAG :

beobachten (bei-Dat)

$01(4$

01

014

beraten mit-Dat

1:SE mit fakultativem Korrelat
Er bemerkte zu dem Problem, er halte es für unlösbar.

$E_{1}$ meist indefinit oder $\mathrm{SE}$.

P1 Du hast die Eltern (hierher) bemüht.

PO Der Arzt bemüht sich (um den Kranken). Ich bemühe mich <darum>, eine gute Prüfung zu machen.

Ich bemühe mich <darum>, daß ich die Arbeit bekomme.

P1 Er beobachtet seine Frau.

Ich habe <es > beobachtet, daß du deine Schwester geschlagen hast.

Das Kind beobachtet <es> genau, wie sein Vater das Auto repariert.

P1 Er beobachtet ihn (beim Schwimmmen).

P1 Der Wirt berät den Gast.

P1 Ich berate meine Pläne mit dir.

FRAG : Die Eltern beraten <es > mit den Kinderm, wohin sie in Urlaub fahren. 


\section{berichten}

berichten

0113

1:SE ohne Korrelat

DASS :

FRAG:

HPTS :

BEMERKUNG :

berichten über - Akk

$O(34$

4:SE mit fakultativem Korrelat

berichten von - Dat

4: SE mit fakultativem Korrelat

$O(34$

DASS :

FRAG:

\section{beruhigen}

beruhigen

O:SE Zum Korrelat vgl. Einleitung 6.3.5.

beruhigen (mit-Dat)

sich/Akk beruhigen

$0(1$

$01(4$

0
P1 Er berichtet (uns) Neues aus der Alten Welt. Er berichtet mir, daß sich etwas ereignet hat. Er berichtet mir, wie sich die Sache ereignet hat. Er berichtet mir, es hat sich etwas ereignet. $E_{1}$ meist indefinit oder SE.

P2 Der Fremde berichtet (mir) über den Unfalz. Mein Freund hat mir <dariber > berichtet, daß er einen Unfall hatte.

Mein Freund hat nur mir <darüber > berichtet, wie es zu diesem Unfall kam.

P2 Er berichtet (mir) von dem Unfall.

Mein Freund hat nur mir <davon> berichtet, daß er in diesen Unfall verwickelt war.

Mein Freund hat mir nie <davon> berichtet, wie es zu diesem Unglück gekommen ist.

P1 Musik beruhigt (mich).

INF+: $\quad$ Es beruhigt mich, dich zu sehen.

DASS: $\quad$ Es bemhigt mich, daß du das gesagt hast.
P1 Hans beruhigt seine Freunde (mit einer Erklärung).

PO Das Meer beruhigt sich. 


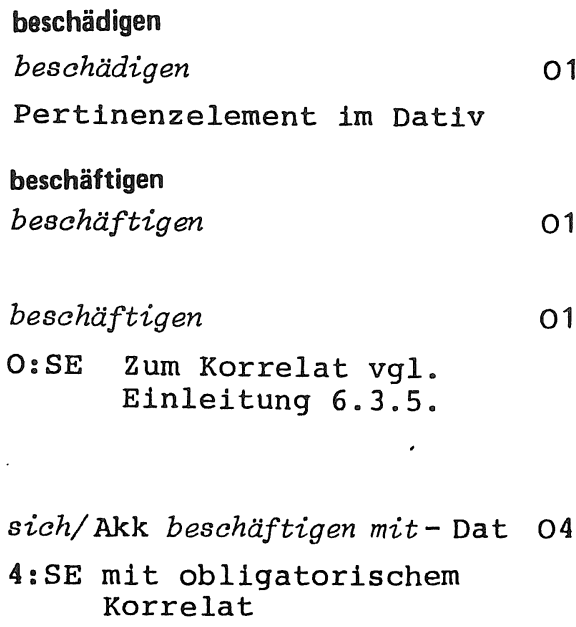

DASS :

FRAG :

INF+ :

FRAG :

\section{beschließen}

beschließen
P1 Der Sturm beschädigte die Häuser. Man hat mir die Brizle beschädigt.

P1 Diese Firma-beschäftigt viele Angestellte. Tante Emma beschäftigt die Kinder.

PO Das Problem beschäftigt den Studenten. Es beschäfigt den Mann, daß seine Frau sich vernachlässigt.

Es beschäftigt den Studenten, wie das Problem zu Zösen ist.

PO Ich beschäftige mich mit Geschichte. Hans beschäftigt sich schon lange domit, dieses Problem zu lösen.

Der Forscher beschäftigt sich schon lange domit, wie dieses Problem zu lösen ist.

P1 Der Personalchef beschloß die Entlassung des Angestelzten.

INF+: Sie beschlossen, ihn zu entlassen.

DASS: $\quad$ Sie beschließen, daß er entlassen wird.

HPTS: Müllers beschließen, sie bleiben zu Hause. 
beschließen mit-Dat

014

4:SE mit obligatorischem Korrelat

INF+:

DASS :

\section{beschreiben}

beschreiben

beschreiben

1:SE mit fakultativem Korrelat

beschreiben (mit-Dat)

\section{beschweren}

beschweren

01

sich/Akk beschweren (bei-Dat) (über - Akk)

4:SE mit obligatorischem Korrelat

01

$01(3$

FRAG :

$01(4$

$014(4$

INF+:

DASS :

\section{besetzen}

besetzen
01
P1 Der Redner beschloß seinen Vortrag mit einem Dankeswort.

Er beschloß seinen Vortrag domit, den Veranstaltern für die gute Organisation zu danken.

Erbeschloß seine Rede domit, daß er den Veranstaltern für die gute Organisation dankte.

P1 Das Flugzeug beschreibt einen Kreis.

P1 Sie beschreibt (mir) den Mantel.

Der Lehrer beschreibt $\langle e s\rangle$ den Schülern noch einmal, wie die Aufgabe zu lösen ist.

P1 Der Schüler beschreibt die Tafel (mit Formeln).

P1 Ich beschwere die Briefe [mit einem Stein].

PO Wir beschweren uns (beim Chef) (über die Löhne).

Wir beschweren uns darüber, schlecht behandelt worden zu sein.

Wir beschweren uns darüber, daß wir schlecht behandelt werden.

P1 Die alte Dame besetzte den freien Platz. Die Studenten besetzen das Haus. 
besetzen mit - Dat

014

besetzen

018

\section{besichtigen}

besichtigen

\section{besinnen}

sich/Akk besinnen

2:SE ohne Korrelat

02

DASS :

FRAG :

sich/Akk besinnen auf-Akk

4:SE mit obligatorischem Korrelat

04

INF+:

DASS :

FRAG :
sich/Akk besinnen
P1 Die Mutter besetzt den Mantel mit Pelz.

Der Regisseur hat die Rolle des Hamlet mit einem schlechten Schauspieler besetzt.

P1 Der Regisseur hat die Rolle des Homlet schlecht besetzt.

P1 Die Schüler besichtigen die Ausstellung.

PO Er besinnt sich eines Besseren.

Er kann sich nicht besinnen, diesen Brief erhalten zu haben.

Nach Zangem Nachdenken besann er sich, daß er diesen Brief erhalten hatte.

Er konnte sich nicht besinnen, wo er den Brief hingelegt hatte.

PO Sie besinnt sich auf ihre Pfichten.

Er kann sich nicht darauf besinnen, das Buch gelesen zu haben.

Er kann sich nicht darauf besinnen, daß er das Buch gelesen hat.

Er kann sich nicht darauf besinnen, wo er das Buch gekauft hat.

PO Die Frau besann sich (anders). 


\section{besorgen}

besorgen

01

\section{bestehen}

bestehen

bestehen

bestehen

bestehen auf-Dat

4:SE mit obligatorischem Korrelat

bestehen aus - Dat

4:SE mit obligatorischem Korrelat

bestehen in - Dat

4:SE mit obligatorischem Korrelat

bestehen vor - Dat

bestehen
INF+:

DASS :

04

04

INF+ :

DASS :

05
P1 Die Hausfrou hat Milch besorgt. Der Bauer besorgt den Hof.

PO Es besteht [noch] Hoffnung.

P1 Siegfried bestand den Kampf.

P1 Der Student besteht (seine Prüfung).

P2 Er besteht auf seinem Recht. Er besteht darauf, sein Recht zu bekommen. Er besteht darauf, daß wir seiner Einladung folgen.

PO Das Haus besteht aus Steinen.

Die Prüfung besteht aus einer Ubersetzung und einer Interpretation.

DASS: Die Prüfung besteht daraus, daß ein Text übersetzt und interpretiert wird.

po Die Prüfung besteht in einer schriftzichen Arbeit. Die Prüfung besteht darin, einen Testbogen auszufülzen.

Die Prüfung besteht darin, daß ein Testbogen ausgefüllt wird.

PO Er kann vor den Augen seiner Familie bestehen.

Po Die Fabrik besteht seit einigen Jahren. 


\section{bestellen}

bestellen

01

bestellen

$1: S E$ ohne Korrelat

013

\section{DASS :}

HPTS :

besteZlen (bei-Dat)

bestellen

$01(4$

016

\section{bestimmen}

bestimmen

1:SE mit fakultativem Korrelat

\section{DASS :}

FRAG :

HPTS :

bestimmen $z u$-Dat

014

4:SE mit obligatorischem Korrelat
P1 Der Bauer bestellt das Feld.

P1 Ein Junge hat mir die Nachricht bestellt. Der Junge hat mir bestellt, daß ich gleich kommen solz.

Der Junge bestellt mir, ich solle in die stadt fahren.

P1 Die Hausfrau bestel.lt Kartoffeln (beim Bauern).

P1 Otto bestellt seine Freundin an den Bahnhof.

P1 Der Lehrer bestimmt den Termin der Prüfung. Der Lehrer hat <es> bestimmt, daß die Prüfung morgen stattfindet.

Die Schulleitung wird <es> morgen bestimmen, wann die Prüfung stattfindet.

Der Lehrer hat 〈es> endgültig bestimmt, die Prüfung findet am Donnerstag statt.

P1 Der Minister hat Herm Mayer zum Chef dieser Abteilung bestimmt.

INF+: Der Chef hat Herrm Mayer dazu bestimmt, während seines Urlaubs das Geschäft zu führen.

DASS: $\quad$ Der Chef hat Herm Mayer dazu bestimmt, daß er während seines Urlaubs das Geschäft führt. 
bestimmen uber - Akk

04

4:SE mit obligatorischem Korrelat

\section{bestrafen}

bestrafen (fur - Akk)

4:SE mit obligatorischem Korrelat

\section{besuchen}

besuchen

\section{betrinken}

sich/Akk betrinken

betrügen

betrügen

betrügen ( um - Akk)

\section{bewegen}

bewegen

bewegen

O:SE Zum Korrelat vgl. Einleitung 6.3 .5 .
P2 Der Vater bestimmt über di.e Zukunft seiner Kinder.

FRAG: Das Gericht bestimmt darüber, ob der Flughafen gebaut werden darf.

P1 Das Gericht bestraft den Verbrecher (für seine Taten) [mit Gefängnis].

DASS: $\quad$ Der Lehrer bestraft den Schüler dafür, daß er zu spät gekommen ist.

P1 Der Vater besucht seinen Sohn (im Krankenhaus).

PO Unser Nachbar hat sich betrunken.

P1 Der Ehemann betrügt seine Frau.

P1 Der Wind bewegt die Blätter.

PO Dein Besuch hat mich (sehr) bewegt.

INF+: $\quad$ Es hat mich sehr bewegt, dich so krank zu sehen.

DASS: $\quad$ Es hat mich sehr bewegt, daß du mich besucht hast. 


\section{bewegen \\ sich/Akk bewegen \\ sich/Akk bewegen 2om-Akk \\ 4:SE mit obligatorischem Korrelat}

sich/Akk bewegen

sich/Akk bewegen

BEMERKUNG :

\section{bewegen \\ bewegen zu-Dat \\ 4:SE mit fakultativem Korrelat \\ 4:SE mit obligatorischem Korrelat \\ BEMERKUNG :}

\section{beweisen}

beweisen

1:SE mit fakultativem Korrelat
0

04

DASS :

FRAG:

018

014

INF+:

DASS :

$01(3$

DASS :

HPTS :
P1 Ich kann den Wagen [kaum] von der Stelle bewegen.

PO Das Blatt bewegt sich.

PO Seine Gedanken bewegen sich um das Mädchen.

Seine Gedanken bewegen sich den ganzen Tag darum, daß er morgen Examen hat.

Seine Gedanken bewegen sich den ganzen Tag darum, wie dieses Problem zu lösen ist.

PO Das Mädchen bewegt sich [nur] in guter Gesellschaft.

Po Das Kind bewegt sich (naturlich).

STAMMFORMEN: bewegen, bewegte, bewegt.

P1 Seine kritischen Worte bewogen mich zum Nachdenken. Er bewog mich <dazu>, ihn zu besuchen.

Er bewog mich dazu, daß ich mich bei ihr für mein schlechtes Benehmen entschuldigte.

STAMMFORMEN: bewegen, bewog, bewogen.

P1 Sein Verhalten beweist (mir) seine Aufmerksamkeit. Dieser Unfall beweist <es> uns wieder, daß diese Straße gefährlich ist.

Dieser Unfall beweist <es> uns wieder, diese Straße ist gefährlich. 


\section{bewerben}

sich/Akk bewerben (bei-Dat)

( $2 \mathrm{~m}-\mathrm{Akk})$

4:SE mit obligatorischem Korrelat

INF+:

\section{bewundern}

bewundern

$1: \mathrm{SE} \quad$ Zum Korrelat vgl. Einleitung 6.3 .5 .

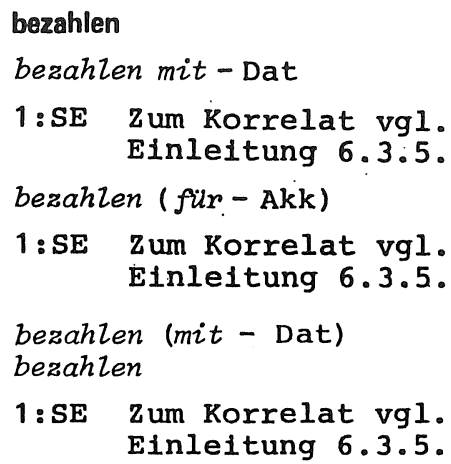

01

DASS :

014

DASS :

$0(1(4$

DASS :

$0(1) 4$

018

\section{DASS :}

\section{beziehen}

beziehen
P1 Der Junge bewundert das Mädchen.

Ich beurndere es, daß du ihm hilfst.

Po Er bewirbt sich (bei dieser Firma) (um den Posten als Abteilungsieiter).

Er bewirbt sich darum, in den Aufsichtsrat gewählt zu werden.

P1 Sie hat ihren Irrtum mit dem Leben bezahlen müssen. sie hat es mit dem Leben bezahlen müssen, daß sie nicht aufgepaßt hat.

P1 Er bezahlt (zwei Mark) (für Zigaretten). Er bezahlt zehn Mark dafur, daß er falsch geparkt hat.

P1 Er bezahlt (das Auto) (mit einem Scheck).

P1 Sie hat ihren Irrtum teuer bezahlen milssen.

Er hat es teuer bezahlen müssen, daß er dem Geschätspartner vertraut hat.

P1 Wir beziehen das Haus. 


beiziehen auf-Akk
beziehen ( mit- Dat)
beziehen
beziehen
sich/Akk beziehen auf-Akk
4: SE mit obligatorischem
$\quad$ Korrelat
sich/Akk beziehen (mit-Dat)
bieten
bieten
bieten
bieten (fü-Akk)
4:SE mit obligatorischem
Korrelat

\section{bitten}

bitten um - Akk

4:SE mit fakultativem Korrelat

\section{4}

$01(4$

016

Q1 (6

04

\section{DASS :}

FRAG :

$0(4$

01

013

$0113(4$

DASS:

$O(14$

INF+:

DASS :

HPTS :
P1 Der'Schüler bezog die Vorschrift auf einen bestimmten Falz.

P1 Mutter bezieht die Betten (mit frischer Wäsche).

P1 Die Bundesrepublik bezieht Erdöl aus Nahost.

PO Hans bezieht ein gutes Gehalt (von seiner Firma).

PO Seine Antwort bezog sich ouf den Brief. Unser Gespräch bezog sich darauf, daß wir wahrscheinlich eine Entscheidung verschieben müssen. Die Frage bezog sich darauf, was wir morgen machen.

PO Der Himmel bezog sich (mit drohenden Wolken).

Po Diese Arbeit bietet keine Schwierigkeiten.

P1 Er bietet ihr ein schönes Leben.

P1 Erbietet (dir) zehntausend Mark (für das Bild). Er bietet Hans hundert Mark dafür, daß er ihm eine Wohnung vermittelt.

P1 Er bittet (seinen Vater) um Hilfe. Er bittet seinen Vater <darum >, ihm zu helfen. Er bittet seinen Vater <darum >, daß er ihm hilft. Er bittet seinen Vater <darum>, er solle ihm helfen. 


\begin{tabular}{|c|c|c|c|}
\hline VERB & SBP & & BEISPIELSATZ \\
\hline bitten (bei-Dat) fur -Akk & $0(44$ & P2 & Er bittet (beim Direktor) für seinen Sohn. \\
\hline \multicolumn{4}{|l|}{ bleiben } \\
\hline bleiben & 013 & PO & Die Erinnerung bleibt (mir). \\
\hline bleiben & 05 & PO & Erblieb im Bett. \\
\hline Pertinenzelement im Dativ & & & Das ist mir im Gedächtnis geblieben. \\
\hline bleiben & 07 & PO & Klaus bleibt Beanter [trotz des niedrigen Gehalts]. \\
\hline bleiben & 08 & $\mathrm{PO}$ & [Noch] bleibt er mihig. \\
\hline bleiben (es) & 7 & PO & [Im Sommer] bleibt es [Zange] Tag. \\
\hline bleiben (es) & 8 & PO & Es bleibt [im Winter länger] dunkel [als im Sommer]. \\
\hline *am Leben bleiben & I & $\mathrm{PO}$ & Der Verunglückte blieb am Leben. \\
\hline \multicolumn{4}{|l|}{ blühen } \\
\hline bzühen & o & PO & $\begin{array}{l}\text { Sein Geschäft blüht. } \\
\text { Die Blumen blühen. }\end{array}$ \\
\hline \multicolumn{4}{|l|}{ bluten } \\
\hline bluten & 0 & PO & Seine Hand blutet. \\
\hline Pertinenzelement im Dativ & & & Mir blutet das Zahnfleisch. \\
\hline \multicolumn{4}{|l|}{ braten } \\
\hline braten & 0 & PO & Die Schnitzel braten. \\
\hline braten & 01 & P1 & Die Kinder braten ein Schnitzel. \\
\hline
\end{tabular}




\section{brauchen}

brauchen

brouchen fur - Akk

01

014

brechen

brechen

Pertinenzelement im Dativ

brechen mit-Dat

04

BEMERKUNG :

\section{brechen}

brechen

Pertinenzelement im Dativ

brechen

BEMERKUNG :

\section{bremsen}

bremsen

\section{brennen}

brennen

Pertinenzelement im Dativ brennen
P1 Ich brauche deine Hilfe.

P1 Der Zug braucht für diese Strecke fünf Stunden.

P1 Er bricht sein Versprechen.

[Beinahe] hätte ich mir den Fuß gebrochen.

P2 Otto hat mit seinen alten Gewohnheiten gebrochen. STAMMFORMEN: bricht, brach, hat gebrochen.

PO Das Eis bricht.

Mir bricht der Fingernagel.

Po Die Sonne bricht durch die Wolken.

STAMMFORMEN: bricht, brach, ist gebrochen.

P1 Berta bremst (das Auto).

PO Das Licht brennt. Euer Hous brennt. Mir brennen die Füße. 


\section{brennen ( zu - Dàt)}

brennen

Pertinenzelement im Dativ

brennen auf - Akk

\section{4:SE mit obligatorischem} Korrelat

brennen

Pertinenzelement im Dativ

bringen
bringen
bringen
bringen

bringen auf - Akk
4: SE mit obligatorischem
Korrelat

\section{$01(4$}

016

04

INF+:

DASS :

06

01

013

013v6

014

INF+:

DASS :
P1 Wir brennen das Holz (zu Kohle).

P1 Die Zigarre brennt ein Loch in die Tischdecke. Er hat mir [mit der 2igarre] ein Loch in den Mantel gebrannt.

PO Ich brenne ouf dein Kommen.

Ich brenne darauf, dich zu sehen.

Das Kind brennt darauf, daß es endlich seine Geschenke auspacken darf.

Po Die Sonne brennt auf die Dünen.

Die Sonne brennt mir ouf den Rücken.

P1 Die Zeitung bringt Neues.

P1 Der Chor bringt dem Direktor ein Ständchen.

P1 Erbringt ihr die Bücher ins Büro.

Der Briefträger bringt ihr einen Brief.

Mitter bringt die Blumen ins Wohnzimmer.

P1 Das Gespräch hat ihn auf einen Ausweg gebracht.

Der Hinweis des Freundes hat ihn darauf gebracht, seinen Aufsatz neu zu schreiben.

Der Hinweis des Freundes brachte ihn darauf, daß er einen Fehler gemacht hatte. 


\section{4:SE mit obligatorischem Korrelat}

bringen über - Akk

bringen um-Akk

bringen zu-Dat

4:SE mit obligatorischem Korrelat

bringen

bringen es auf-Akk .

bringen es zu-Dat

4:SE mit obligatorischem Korrelat

\section{BEMERKUNG :}

*an den Mann bringen

*in Aufregung bringen

*in Begeisterung bringen uber-Akk

*i.n Bewegung bringen

*in Ekstase bringen

*in Erfahrung bringen
014

$\mathrm{T}$

FRAG:

014

014

INF+:

DASS :

016

04

04

INF+:

DASS :

T

$\mathrm{T}$
Der Hinveis des Freundes brachte ihn darauf, wie er seine Arbeit verbessern könnte.

P1 Er bringt Unglück über die Familie.

P1 Der Dieb brachte mich um mein Geld.

P1 Der Lehrer bringt die Schüler zum Schweigen.

Mit Mühe brachte er ihn dazu, über diesen Vorfall zu schweigen.

Mit Mühe konnte er ihn dazu bringen, daß er über den Vorfall schwieg.

P1 Ich bringe Carola an die Bahn.

Po Dieses Ehepaar hat es ouf acht Kinder gebracht.

PO Er hat es zu etwas gebracht.

Er brachte es dazu, von allen anerkannt zu werden.

Er hat es endlich dazu gebracht, daß sie ihn heiratet. $\mathrm{E}_{4}$ meist indefinit oder SE.

P1 Hans bringt die Ware an den Mann.

P1 Die Nachricht brachte seinen Bruder in große Aufregung.

P1 Der Architekt brachte den Bauherrn nicht gerade in helle Begeisterung über seine Pläne.

P1 Der Mann brachte das Rad in Bewegung.

P1 Die Beatles bringen die Jugend in Ekstase.

P1 Er brachte die Hausnumer in Erfahrung. 
*in Erinnerung bringen.

*in Fahrt bringen

*in FZUß bringen

*in Gang bringen

* in Gefahr bringen

*in seine Gewalt bringen

*in seine Hände bringen

*in Ordnung bringen

*ins Rollen bringen

*ins Schwitzen bringen

* in Schwung bringen

*ins Spiel bringen

*in Stimmung bringen

*in Verbindung bringen mit-Dat

*in Verlegenheit bringen über - Akk

*ill Wut bringeil mit: - Dat

*ir wui wringei äbtor - isk k
P1 Er brachte ihm seine Schulden in Erinnerung.

P1 Seine Unverschömtheit bringt mich in Fahrt.

P1 Er brachte das Gespräch in Fluß.

P1 Der Mechaniker bringt das Auto wieder in Gang.

P1 Die Rücksichtslosigkeit des Autofahrers bringt den Fußgänger in große Gefahr.

Seine leichtfertigen Reden brachten sie in Gefahr, unüberlegt zu handeln.

P1 Sie bringen die Geiseln in ihre Gewalt.

P1 Er brachte die Geheimpapiere in seine Hände.

P1 Die Frau brachte das Haus in Ordnung.

P1 Er bringt den Stein ins Rolzen.

P1 Die Prüfung brachte ihn ins Schwitzen.

P1 Die Band brachte die Party in Schuring.

P1 Er brachte ein neues Problem ins Spiel.

P1 Der Alkohol bringt die Gäste in Stimmung.

P1 Das Gericht bringt die Tat mit seiner Erziehung in Verbindung.

P1 Er brachte sie in große Verlegenheit über ien ungerechtfertigten Vorwurf.

P1 Der Junge bringt seine Eltern in Wut nir jeinen Streicinen.

P1 Hans bringt seinen :ater in Wut riber Kle inigkesten. 
*in Zuscommenhang bringen mit-Dat

*ins Zwielicht bringen

*unter Kontrolle bringen

*zur Sprache bringen

*zur Verzweiflung bringen

\zur Welt bringen

*weit bringen es auf - Dat

*weit bringen es in - Dat

*weit bringen es mit-Dat

*an sich bringen

*hinter sich bringen

*mit sich bringen

*uber sich bringen
P1 Er bringt den Unfalz in Zusammenhang mit seiner Müdigkeit.

P1 Seine Kontakte zu dem Angeklagten brachten ihn ins Zwielicht.

P1 Die Feuerwehr brachte den Brand unter Kontrolle.

P1 Der Betriebsrat bringt ein heikles Problem zur Sprache.

P1 Seine Fragen bringen mich zur Verzweiflung.

P1 Die Frau brachte ein gesundes Mädchen zur Welt.

PO Dieser Forscher hat es sehr weit auf dem Gebiet der Atomphysik gebracht.

PO Er hat es im Leben weit gebracht.

In den naturwissenschaftlichen Fächern hat dieser Schüler es schon ganz schön weit gebracht.

PO Man bringt es im Beruf oft sehr weit mit Sturheit und Durchsetzungsvermögen.

PO Der Dieb brachte zehntausend Mark an sich.

PO Ich bringe die Prüfung hinter mich.

Po Diese Aufgabe bringt viel Arbeit mit sich.

Po Er brachte die Lüge nicht über sich. 


\section{buchstabieren}

buchstabieren

$0(1$

\section{bürsten}

bürsten

Pertinenzelement im Dativ bürsten

Pertinenzelement im Dativ
P1 Der Schüler buchstabiert (seinen Namen).

P1 Marlies bürstet ihrem kleinen Bruder das Haar. Das Mädchen bürstet sich das Haar.

P1 Karin bürstet ihrem Bruder die Haare aus der Stirn. Karin büstet sich die Haare aus der Stirn. Karin bürstet den Schmutz von ihren Schuhen. 


\section{dankèn}

danken

1:SE Zum Korrelat vgl. Einleitung 6.3.5.

danken ( für - Akk)

4:SE mit fakultativem Korrelat

013

DASS :

dauern

dauern

01

dauern

01

O:SE Zum Korrelat vgl. Einleitung 6.3.5.

douern bis

\section{denken}

denken

1:SE ohne Korrelat

INF+ :

DASS :

HPTS :

BEMERKUNG :

denken an - Akk
P1 Deine Hilfe dankt dir niemand. Niemand dankt es ihr, daß sie ihnen geholfen hat.

P2 Ich danke dir (für deine Hilfe).

Ich danke dir <dafür >, daß du gekommen bist.

PO Die Krankheit dauerte fünf Monate.

PO Er dauert mich.

DASS: $\quad$ Es dauert mich, daß du krank bist.

Po Das Semester douert bis Mitte Juli.

PO Hans denkt (etwas anderes als ich). Ich dachte, dich hier zu finden. Ich dachte, $d a \beta$ du nicht hier seist. Ich dachte, du seist hier. $\mathrm{E}$, meist indefinit oder SE. P2 Ich denke an Piroschka. 


\section{$4: S E$ mit obligator ischem Korrelat}

\author{
denken über - Akj \\ denken von -Dat. \\ denken \\ sich/Dat denken \\ 1:SE mit fakultativem \\ Korrelat.
}

048

048

08

01

INF+:

DASS :

FRAG:

8

DASS :

FRAG :

sich/Dat denken

018

$01(3$

$0(16$

diktieren

0

dröngen

$0(14$
Ich denke daran, in die Stadt zu gehen.

Ich denke daran, daß Gabi bald kommen wird.

Ich denke immer wieder daran, wie schön der UrZaub war.

Po Er denkt schlecht über seine Frau.

PO Er denkt schlecht von seiner Frau.

PO Er denkt wie ich.

PO Ich denke mir eine Zahl.

Er hat $\langle e s\rangle$ sich gedacht, die Arbeit schnell zu erledigen.

Er hat <es> sich nicht gedacht, daß die Arbeit so schwierig wird.

Ich kann <es> mir schon denken, warum du nicht kommen willst.

po Er denkt sich die Sache leicht.

P1 Der Verwaltungsleiter diktiert (seiner Sekretärin) einen Brief.

P1 Er diktiert (seine Memoiren) auf Band.

PO Die Zeit drängt.

P1 Er drängt (mich) zur Entscheidung. 


\section{4:SE mit fakultativem} Korrelat

\section{drängen}

drängen auf - Akk

4:SE mit obligatorischem Korrelat

drängen

sich/Akk drängen

\section{drehen}

drehen

drehen

Pertinenzelement im Dativ drehen

sich/Akk drehen um-Akk

4:SE mit obligatorischem Korrelat

sich/Akk drehen
INF+:

DASS :

016

04

INF+:

DASS :

06

06

01

016

016

04

DASS :

FRAG:
Er drängt mich 〈dazu〉, mich schnell zu entscheiden. Er drängt mich 〈dazu〉, daß ich mich schnell entscheide.

P1 Der Boxer drängte seinen Gegner in eine Ecke.

P2 Er drängt ouf Antwort.

Er drängt darauf, eine Antwort zu bekommen.

Er drängt darauf, daß er eine Antwort bekommt.

P2 Die Menge drängt zu den Ausgängen.

P2 Er drängt sich nach vorn.

P1 Herr Bauer dreht einen Film. Der Apotheker dreht Pillen.

P1 Ich drehe meine Haare um die Finger. Sie drehen ihm den Arm ouf den Rücken.

PO Der Wind dreht (nach Osten).

PO Ihr Gespräch dreht sich um ein schwieriges Problem. Ihr Gespräch dreht sich darum, daß morgen Feiertag ist.

Ihr Gespräch dreht sich darum, ob der Vertrag geschlossen wird.

PO Der Wind hat sich (nach Osten) gedreht. 


\begin{tabular}{|c|c|c|c|c|}
\hline VERB & SBP & & & BEISPIELSATZ \\
\hline $\begin{array}{l}\text { ^sich/Akk im Kreise drehen } \\
\text { drucken }\end{array}$ & $I$ & & po & Ihr Gespräch dreht sich im Kreis. \\
\hline drucken & 01 & & P1 & Der Verlag druckt ein Buch. \\
\hline drucken & 016 & & P1 & Die Schüler drucken Bilder auf das Papier. \\
\hline \multicolumn{5}{|l|}{ drücken } \\
\hline drücken & 01 & & P1 & Er drückt die Klingel [anhaltend]. \\
\hline drücken & $0(1$ & & $\mathrm{PO}$ & Die Schuhe drücken (mich). \\
\hline drücken & 016 & & P1 & Emma drückt Fritz an die Brust. \\
\hline drücken & 06 & & P2 & Der Gast drückt auf die Klingel. \\
\hline sich/Akk drücken ( $2 m-A k k)$ & $0(4$ & . & PO & Er drückt sich (um den Dank). \\
\hline sich/Akk drücken (vor-Dat) & $0(4$ & & PO & Er drückt sich (vor der Arbeit). \\
\hline $\begin{array}{l}\text { 4:SE mit obligatorischem } \\
\text { Korrelat }\end{array}$ & & INF+: & & Er drückt sich davor, arbeiten zu müssen. \\
\hline $\begin{array}{l}\text { ^die Hand drücken } \\
\text { duschen }\end{array}$ & I & & PO & Er drückt seinem Freund die Hand. \\
\hline$\langle\operatorname{sich} /$ Akk $\rangle$ duschen & 0 & & PO & Meine Schvester duscht <sich> [gerade]. \\
\hline
\end{tabular}




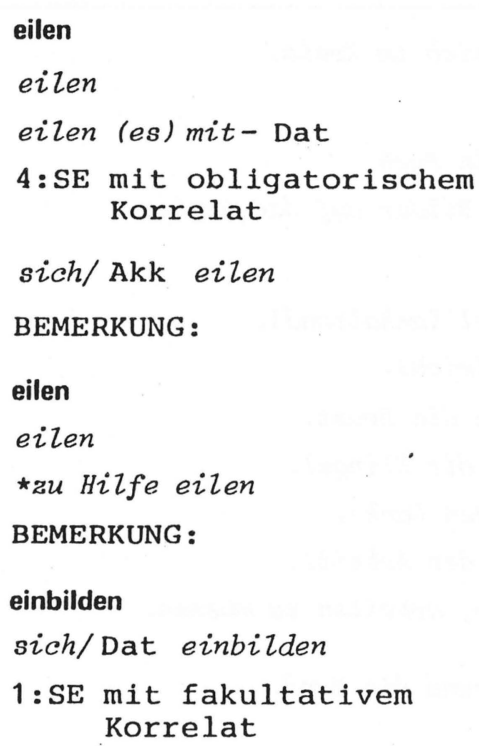

BEMERKUNG :

\section{einbilden}

sich/Dat einbilden

1:SE mit fakultativem Korrelat

013

34

INF+:

DASS :
PO Die Sache eilt (mir).

Po Mit der Arbeit hat es dem Chef geeilt. Es eizt ihm damit, die Arbeit zu beginnen. Es eilt ihm domit, daß diese Arbeit erledigt wird.

PO Er soll sich eilen. STAMMFORMEN: eilen, eilte, hat geeilt.

PO Das junge Mädchen eilt in den Garten.).

P2 Der Arzt eilt dem Kranken zu Hilfe. STAMMFORMEN : eilen, eilte, ist geeit.t.

PO Seine Großmutter bildet sich die Krankheit ein. INF+:

DASS :

HPTS :

\section{BEMERKUNG :}

sich/Dat einbilden (auf-Akk) 01 ( 4 4:SE mit obligatorischem Korrelat Seine Großmutter bildet 〈es〉 sich ein, krank zu sein.

Seine Großmutter bildet <es> sich ein, daß sie

Seine Großmutter bildet <es > sich ein, sie sei krank.

$\mathrm{E}_{1}$ meist indefinit oder SE.

Po Dubildest dir zuviel (auf deinen Erfolg) ein. Der Mann bildet sich nichts darauf ein, Erfolg zu haben. krank sei. 


\section{4:SE mit obligatorischem Korrelat}

BEMERKUNG :

\section{einkaufen}

einkaufen

011

sich/Akk einkaufen in - Akk

04

\section{einladen}

einZaden zu - Dat

$01 v 4$

\section{4:SE mit fakultativem} Korrelat

einladen

\section{einpacken}

einpacken (in - Akk)

\section{einschlafen}

einschlafen

O

Pertinenzelement im Dativ
DASS: Hein Freund bildet sich nichts darauf ein, daß er Erfolg hat.

$\mathrm{E}_{1}$ meist indefinit.

P1 Die ifutter kauft (Lebensmittel) ein.

PO Er kauft sich in das Geschäft ein.

P1 Hans lädt Gisela zum Essen ein.

Hans lädt Gisela ein.

Hans lädt zum Essen ein.

INF+:

Hans lädt Gisela 〈dazu> ein, mi.t ihm ssen zu gehen.

P1 Hans lädt Gisela (ins Theater) ein.

P1 Gabi packt das Buch (in Geschenkpapier) eir.

PO Die Kinder schiufer: eir.

Der Eriefwechsei sahtraft eirn.

Mir ist die Hand eingesintafe'. 


\section{einschreiben}

einschreiben (in-Akk)

$01(4$

\section{einsteigen}

einsteigen in - Akk

04

einsteigen

016

entlassen

entzassen

entlassen

\section{entscheiden}

entscheiden

1 :SE ohne Korrelat

entscheiden auf - Akk

entscheiden ( Uber-Akk)

4:SE mit fakultativem Korrelat

sich/Akk entscheiden für-Akk
P1 Der Student schreibt sich [an der Universität] (in eine Liste) ein.

P2 Peter steigt in das Geschäft seines Vaters ein.

P2 Die Leute steigen (in den Zug) ein.

P1 Der Chef entläßt den Angestellten.

P1 Der Lehrer entläßt die Schüler (in die Pause).

P1 Hans entscheidet den Streit.

INF+: Hans entscheidet, den Vorschlag anzunehmen.

DASS: Hans entscheidet, daß wir den Vorschlag annehmen.

FRAG: Hans entscheidet, ob wir noch länger. bleiben.

HPTS: Hans entscheidet, wir gehen in die Stadt.

P2 Der Richter entscheidet auf Gefängnis.

P2 Das Los entscheidet (über den Gewinn).

FRAG : Das Los entscheidet <darüber >, ob wir gewinnen.

PO Wir entscheiden uns für dieses Auto. 
4: SE mit fakultativem Korrelat

sich/Akk entscheiden gegen-Akk

4: SE mit obligatorischem Korrelat

\section{entschließen}

sich/Akk entschließen für -Akk

sich/Akk entschließen zu - Dat

4:SE mit fakultativem Korrelat

\section{entschuldigen}

entschuldigen

sich/Akk entschuldigen

( für - Akk) (bei-Dat)

4:SE mit obligatorischem Korrelat

\section{enttäuschen}

enttäuschen
O $14(4$

INF+:

DASS :

HPTS :

INF+:

DASS :

INF+

\section{DASS :}

01
Wir entscheiden uns <dafür>, dieses Auto zu kaufen.

Wir entscheiden uns <dafür>, daß wir dieses Autc kaufen.

Wir haben uns <dafür> entschieden, wir kaufen dieses Auto.

PO Wir entscheiden uns gegen diesen Plan.

Wir entscheiden uns dagegen, dieses Auto zu kaufen.

Der Redner hat sich dagegen entschieden, daß die Diskussion fortgesetzt wird.

PO Der Student entschließt sich für diese Universität.

PO Meine Freunde entschlossen sich zu dieser Reise. Wir haben uns 〈dxzu> entschlossen, den Vorschlag anzunehmen.

P1 Die Mutter entschuldigte das Verhalten ihres Sohnes.

po Ich entschuldige mich (bei meinem Vater) (für mein Verhalten).

Ich entschuldige mich bei meinem Vater dafür, daß ich zu spät gekommen bin.

P1 Die Verfilmung seines Buches hat den Autor enttäuscht. 
O:SE Zum Korrelat vgl. Einleitung 6.3.5.

INF+:

DASS :

\section{entwickeln}

entwickeln

entwickeln

sich/Akk entwickeln zu-Dat

\section{ereignen}

sich/Akk ereignen

\section{erfahren}

erfahren

01

erfahren (von - Dat)

$1: \mathrm{SE}$ mit fakultativem Korrelat

DASS :
Es enttäuschte die Eltern, ihren Sohn in schlechter Cesellschaft zu sehen.

Es enttäuschte die Eltern, daß ihr Sohn einen anderen Beruf ergriffen hatte.

P1 Der Fotograf entwickelt einen Film.

Hans entwickelt Maschinen.

Hans entwickelt große Kräfte.

P1 Der Arbeiter entwickelt dem Chef einen Plan.

PO Das Land entwickelt sich zu einem Industriestaat.

PO Das Unglück ereignete sich vor diesem Haus. Das Unglück ereignete sich um fünf Uhr.

Po Er erfuhr viel Liebe. Er erfährt eine gute Behandlung.

PO Er erfuhr ein Geheimnis (von mir). Er hat <es> nie erfahren, daß sein Freund ihn betrogen hat.

Erst jetzt hat er <es> erfahren, wann sein Vater gefallen ist.

HPTS: $\quad$ Ich habe <es> eben erfahren, der Minister ist zurückgetreten. 
erfahren von - Dat

04

4:SE mit fakultativem Korrelat

\section{erfinden}

erfinden

01

\section{erholen}

sich/Akk erholen (von - Dat)

$0(4$

\section{erinnern}

erinnern an-Akk

4:SE mit obligatorischem Korrelat

DASS :

FRAG :

sich/Akk erinnern (an-Akk)

$\mathrm{O}(4$

4:SE mit fakultativem Korrelat
PO [Erst spät] habe ich von deiner Abfahrt erfahren. Erst spät habe ich <davon> erfahren, daß du weggefahren bist.

P1 Wankel erfand einen Motor.

Der kleine Junge erfindet eine Ausrede.

Po Der Sportler erholt sich (von seinem Wettkampf).

P1 Diese Mode erinnert (mich) an frühere Zeiten. E' erinnert mich daran, daß ich ihm schreiben wollte.

Ich erinnere ihn daran, wo wir uns zum ersten Mal getroffen haben.

PO Ich erinnere mich (an das gestrige Essen).

Ich kann mich nicht <daran〉 erinnern, den alten Mann schon einmal gesehen zu haben.

DASS: $\quad$ Ich kann mich nicht <daran > erinnerm, daß wir uns verabredet hatten.

FRAG :

Ich erinnere mich nicht <daran >, wo ich die Tasche hinge legt habe.

HPTS :
Ich kann mich genau <daran> erinnern, ich habe den Mann gestern gesehen. 


\section{erkälten}

sich/Akk erkälten

0

erkennen

erkennen

$1:$ SE mit fakultativem Korrelat

01

\section{DASS :}

FRAG :

HPTS :

erkennen (an - Dat)

$01(4$

4:SE mit obligatorischem Korrelat

erkennen auf - Akk

04

\section{erklären}

erkzären

01

erklären

1:SE ohne Korrelat

erklären
PO Ich habe mich erkältet.

P1 Robert erkennt das Problem.

Er hat <es > sofort erkannt, daß er das Spiel verZoren hat.

Er hat <es> schnell erkannt, wie das Problem zu Zösen ist.

Der Arzt hat <es > schnell erkannt, der Verletzte muß operiert werden.

P1 Die Mutter erkennt ihren Sohn (an den langen Haaren).

DASS: $\quad$ Man erkennt ein Polizeiauto daran, daß es Blaulicht hat.

P2 Das Gericht erkannte auf Gefängnis.

P1 Die Regierung erklärt den Ausnahmezustand.

P1 Der Lehrer erklärt (dem Schüler) die Aufgabe.

FRAG: Der Lehrer mußte dem Schüler mehrmals erklären, wie ein Motor funktioniert.

P1 Die Römer erklärten (den Kathagern) den Krieg. Der Minister erklärte (dem Kanzler) seinen Rücktritt. 
$1:$ SE ohne Korrelat

erklären für - Adj

erklären

014

$01(5$

1:SE ohne Korrelat

erlauben

erlauben

1:SE mit fakultativem Korrelat
INF+:

DASS :

HPTS :

INF+

DASS :

HPTS :

INF+:

DASS :
Wir erklärten immer wieder, nicht einverstanden zu sein.

Der Angeklagte erklärte immer wieder, daß er unschuldig sei.

Der Lehrer erklärte den Schülern immer wieder, ihr Verhalten sei nicht tragbar.

P1 Das Gericht erklärt den jungen Mann für mündig.

P1 Der Minister hat (vor der Presse) nichts Neues erklärt.

Der Minister hat vor der Presse erklärt, in die USA zu wollen.

Der Minister hat vor der Presse erklärt, daß er in die USA reisen werde.

Der Minister hat vor der Presse erklärt, er werde in die USA reisen.

$\mathrm{E}_{1}$ meist indefinit oder SE.

Po Der junge Mann hat sich seiner Angebeteten erklärt.

PO Unsere Arbeit erlaubt keine weitere Verzögerung.

P1 Der Vater erlaubt seiner Tochter eine Urlaubsreise. Der Vater erlaubt 〈es > seiner Tochter, in Urlaub zu fahren.

Der Vater erlaubt <es > seiner Tochter, daß sie nach Italien fährt. 


\author{
eröffnen \\ eröffnen \\ eröffnen \\ 1:SE mit fakultativem \\ Korrelat
}

01

$1:$ SE mit fakultativem Korrelat erreichen

P1 Herr Schmidt eröffnet die Konferenz. Die Brüder eröffnen ein Geschäft.

P1 Wir haben ihm die Schwierigkeiten unserer Situation eröffnet.

DASS: $\quad$ Sie eröffnet <es > ihrem Freund, daß sie ein Kind bekommt.

FRAG: $\quad$ Sie eröffnet $\langle e s\rangle$ ihrem Freund, wann sie ihr Kind bekommt.

HPTS: Sie eröffnet <es > ihrem Freund, sie bekomme ein Kind.

$\mathrm{E}_{1}$ meist $\mathrm{SE}$.

P1 Ich erreichte den Zug. Die Post erreicht den Empfänger.

P1 Der Mieter erreicht die Verlängerung seines Vertrages.

INF+: Der Student hat <es> erreicht, von seinen Eltern mehr Geld zu bekommen.

DASS : Der Student hat 〈es> erreicht, daß ihm seine Eltern mehr Geld geben.

\section{erschrecken}

erschrecken
P1 Das Unglück erschreckte die Bevölkerung. 


\section{$0: S E \quad Z$ um Korrelat vgl.} Einle1tung 6.3.5.

\section{BEMERKUNG :}

\section{erschrecken}

erschrecken (uber - Akk)

4:SE mit obligatorischem Korrelat

erschrecken (vor - Dat)

4:SE mit obligatorischem Korrelat

sich/Akk erschrecken

BEMERKUNG :

\section{BEMERKUNG :}

\section{erwarten}

erwarten

01

emuarten (von - Dat)

1:SE mit fakultativem Korrelat

\section{erzählen}

$01(4$

\section{INF+:}

DASS :

INF+:

DASS :

INF+:

DASS :

DASS :

erzählen

$01(3$
Es erschreckte die Zuschauer sehr, das Unglück zu sehen.

Es hat alle erschreckt, daß dies passierte.

STAMMFORMEN : erschrecken, erschreckte, hat erschreckt.

Po Die Eltern exschraken (über die Nachricht).

Der Arzt erschrak darilber, den Kranken so elend zu sehen.

Der Arzt erschrak darüber, daß der Zustand des Kranken sich so sehr verschlechtert hatte.

Po Der Verbrecher erschrickt (vor dem Hund).

Der Angestellte erschrak davor, daß er mehr Verantwortung bekommen sollte.

PO [Gesterm] habe ich mich [furchtbar] exschrocken. STAMMFORMEN : erschrecken, erschrak, ist erschrocken. Ausnahme beim Refl.: hat ex'schrocken.

STAMMFORMEN : erschrecken, erschrak, ist erschrocken.

P1 Familie Schmitz erwartet Gäste.

PO Die Kinder erwarten (von den EZtem) ein Geschenk. Die Kinder erwarten 〈es > von den Elterm, ein $\mathrm{Ge}-$ schenk zu bekommen.

Sie erwarten < es >, daß sie etwas bekommen.
P1 Die Tante erzählt (ihrem Neffen) eine Geschichte. 
1:SE ohne Korrelat

erzählen ous - Dat

eraählen uber - Akk

erzählen von - Dat

4:SE mit fakultativem Korrelat

\section{erziehen}

erziehen ( $z u$ - Dat)

$4:$ SE mit obligatorischem Korrelat

$01(4$

$0(34$

$\mathrm{O}(34$

O $(34$

DASS :

FRAG :

DASS :

FRAG :

HPTS :

INF+ :

DASS :

\section{essen}

essen

essen

sich/Akk sattessen
Die Tante erzählt ihrem Neffen, daß sie einmal in den Fluß gefallen ist.

Hans erzahlte mir, warum er gestern in den Bach gefallen ist.

Franz erzählte dem Polizisten, es sei ein Unfall geschehen.

po Die Tante erzählt (ihrem Neffen) aus ihrem Leben.

P2 Der Opa erzählt (seinen Enkeln) uber sein Leben.

P2 Der Chef erzählt (uns) von seinen Plänen.

Der Chef erzählt uns <davon>, daß er Großes plant. Der Chef erählt uns <davon〉, was er plant.

P1 Die Eltem erziehen die Kinder (zur Vorsicht im Verkehr).

Die Eltern erziehen ihre Kinder dazu, auf der Straße vorsichtig au sein.

Die Eltern erziehen ihre Kinder dazu, daß sie auf die Verkehrszeichen achten.

P1 Die Kinder essen (Kartoffe $l n$ ).

P1 Wir essen den Teller Zeer. Die Kinder essen uns arm.

po Peter iBt sich satt. 


\section{fahren}

fahren

01

fahren

BEMERKUNG :

\section{fahren}

fahren

fahren

fahren

01

06

Pertinenzelement im Dativ fahren

*aus der Haut fahren

BEMERKUNG :

\section{fallen}

fallen durch - Akk

fallen

fazlen

Pertinenzelement im Dativ fallen
04

$0<5$

06

$0(6$

P1 Hans fährt einen Sportwagen.

Fangio hat einen neuen Rundenrekord gefahren.

P1 Die Firma Meier fährt unsere Mobbl nach Homburg. STAMMFORMEN: fahren, fuhr, hat gefahren.

Po Mein Auto fährt [wieder].

PO Der Busfahrer fährt diese Strecke [schon Zange].

PO Der Zug fährt durch ein langes Tunnel. Er fuhr ihr durchs Haar.

P2 Hans fährt nach Homburg.

PO Der Beante fährt leicht aus der Haut. STAMMFORMEN : fahren, fuhr, ist gefahren.

PO Der Student ist durch die Prüfung gefallen.

PO Hans ist (bei Stalingrad) gefallen.

PO Das Kind fälzt in den Fluß. Das Haar fallt ihm bis ouf die Schultern.

PO Die Temperatur ist (auf minus zwanzig Grad) ge- 
*ins Gewicht fallen

*in die Hände fallen

I

I

*in Ohnmacht fallen

*in Schlaf fallen

*in den Schoß fallen

*ins Wort fallen

*zur Last fallen

\section{fehlen}

fehzen

fehzen

fehlen (es) an-Dat

\section{feiern}

feierm

feiern

krankfeierm

\section{fernsehen}

fermsehen
I

I

I

I

I

O 5

01

O 1

0

0
PO Diese Information fällt für die Entscheidung nicht ins Gewicht.

PO Ein lange gesuchtes Buch fiel mir gestern wieder in die Hände.

Po Die Frau fiel bei der Nachricht vom Tode ihres Mannes in Ohnmacht.

Po Der betäubte Patient fiel in tiefen Schlaf.

PO Das Examen fiel dem Studenten in den Schoß.

P2 Peter fällt dem Lehrer ins Wort.

PO Das unmhige Kind fällt den Elterm zur Last.

Po Der Hund fehlt mir [sehr].

Po Der Lehrer fehlt (in der Schule).

PO Es fehlt (mir) an Geld.

P1 Die Firma feiert die Jubilare.

P1 Die Gäste feiem (Karls Geburtstag).

P2 Der Arbeiter feiert krank.

P2 Meine Elterm sehen [abends manchmal] ferm. 


\section{finden}

finden

finden bei - Dat

4:SE mit obligatorischem Korrelat

\section{BEMERKUNG :}

finden

finden

$1: \mathrm{SE}$ Zum Korrelat vgl. Einleitung 6.3.5.

finden

sich/Akk finden in - Akk

sich/Akk finden zu-Dat
018

INF+:

DASS :

FRAG :

09

9: DASS :

HPTS :

04

INF+:

DASS :

P2

04
P1 Der Anwalt fand keine weiteren Argumente.

Po Ich finde nichts Schlimmes dabei.

Ich finde nichts dabei, schon um sechs Uhr aufzustehen.

Die Eltern finden nichts dabei, daß ihre Tochter kurze Kleider trägt.

$\mathrm{E}_{1}$ meist indefinit.

P1 Ich fand den Nagel (unter dem Schronk).

PO Er findet das Buch interessant.

Viele Leute finden es schwierig, dieses Problem zu erklären.

Viele Leute finden es falsch, daß sie so viele Steuern zahlen müssen.

Der Lehrling findet es falsch, wie er behandelt wird.

Ich finde, daß Klaus recht hat.

Ich finde, Klaus hat recht.

PO Er findet sich in sein Unglück.

po Jemand findet sich zu dieser Arbeit. 


\section{fliegen}

fliegen

01

BEMERKUNG :

\section{fliegen}

fliegen

fliegen

fliegen

06

Pertinenzelement im Dativ

fliegen

016

BEMERKUNG :

\section{fliehen}

fliehen

fliehen

01

fliehen (vor-Dat)

O $14(6$

\section{folgen}

folgen

BEMERKUNG :

\section{folgen}

folgen

folgen
P1 Hans fliegt eine Sportmaschine.

STAMMFORMEN: fliegen, flog, hat geflogen.

PO Meine Haare fliegen [im Wind].

P1 Das. Flugzeug fliegt die Strecke Hannover-Berlin.

Po Der Ball flog iber die Mauer.

sie fliegt ihm an den Hals.

Po Der Vogel fliegt (nach Süden). Hans fliegt [im Jumbo-Jet] (nach Mailand). STAMMFORMEN: fliegen, flog, ist geflogen.

PO Die Zeit flieht.

PO Karin flieht die Arbeit.

P2 Der Verbrecher flieht (vor der Polizei) (ins Aus(and).

P2 Der Hund folgt seinem Herrn [aufs Wort]. STAMMFORMEN: folgen, folgte, hat gefolgt.

PO Dem Frühling folgt der Sommer.

P2 Er folgt ihr (ins Ausland). 
folgen auf - Akk

04

folgen ous - Dat

04 O:SE Zum Korrelat vgl.
Einle1tung 6.3 .5$.

4: SE mit obligatorischem Korrelat

BEMERKÜNG :

\section{fotografieren}

fotografiexen

\section{fragen}

fragen

1:SE ohne Korrelat

BEMERKUNG :

fragen nach - Dat

FRAG :

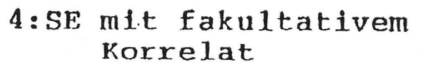

FRAG: po Auf jede Nacht folgt ein Morgen.

Jakob der Zweite folgte auf Karl den Zweiten.

PO Ein mathematischer Beweis folgt aus seinen Axiomen.

DASS: $\quad$ Es folgt eindeutig aus diesen Umständen, daß er sich so verhalten mußte.

FRAG : $\quad$ Es wird aus der Untersuchung folgen, ob Fahrlässigkeit vorliegt oder nicht.

DASS: Die Richtigkeit seiner Behauptungen folgt daraus, daß alles eingetroffen ist.

STAMMFORMEN: folgen, folgte, ist gefolgt.

P1 Almut fotografierte (den Brunnen).

P1 Ich habe dich etwas gefragt.

lch habe dich gefragt.

Ich habe etwas gefragt.

Tch habe dich gefragt, wann du kommen kannst.

Unpersönliche $E_{1}$ meist indefintt oder SE.

P1 Die Housfrou fragt (den Briefträger) nach seiner Tochter.
Die Hausfrau fragt den Briefträger <danach>, wie es seiner Tochter geht. 
fragen wn - Akk

$01 v 4$

09

9: FRAG :

\section{fressen}

fressen

- fressen

Pertinenzelement im Dativ fressen an - Dat

\section{freuen}

freuen

O:SE. Zum Korrelat vgl. Einleitung 6.3.5.

sich/Akk freuen

sich/Akk freuen an - Dat

4: SE mit fakultativem Korrelat

DASS :

INF+ :
P1 Ich habe ihn um Rat gefragt.

Ich habe ihn gefragt.

Ich habe um Rat gefragt.

PO

Er fragt sich, ob diese Geschichte stimnt.

P1 Dieser Hund frißt (Fisch).

p1 Die Säure hat ein Loch in die Tischplatte gefressen. Die Säure hat mir ein Loch in den Kittel gefressen.

PO Der Kummer friBt an ihr.

PO Dein Erfolg freut uns.

INF+: Es freut mich, dich glücklich zu sehen. Es freut mich, daß du so darüber denkst.

PO Er freut sich des Lebens.

PO Günther freut sich om Glück anderer. Günther freut sich 〈daran〉, andere glücklich zu sehen.

DASS: Günther freut sich <daran>, daß andere glücklich sind. 
sich/Akk freuen ouf - Akk

4: SE mit fakultativem Korrelat

Po Die Kinder freuen sich auf die Ferien.

INF+:

DASS :

sich/Akk freuen über-Akk

04

4: SE mit fakultativem Korrelat

\section{frieren}

frieren (es)

frieren

frieren

1

\section{frisieren}

frisieren

Pertinenzelement im Dativ

frisieren

01
Die Kinder freuen sich 〈darauf〉, die Tante bald zu sehen.

Die Kinder freuen sich <darauf>, daß sie ihre Tante bald sehen werden.

po Carola freut sich über ihren Erfolg.

Sie freut sich <darüber>, die Prilfung bestanden zu haben.

Sie freut sich <darüber>, daß sie die Prüfung bestanden hat.

Po Es friert.

PO Er friert (an Händen und Füßen).

PO Es friert ihn.

Ihn friert.

P1 Der Frisör frisiert das Haar der Kundin.

Bärbel frisiert ihre Puppe.

Bärbel frisiert sich.

Er frisiert ihr das Haar.

P1 Die Firma frisiert die Bilanz.

Manfred frisiert seine Yomaha. 


\section{frühstücken}

frühtücken

018

\section{fühlen}

fühlen

01

Pertinenzelement im Dativ $1: \mathrm{SE}$ ohne Korrelat

1:SE mit fakultativem Korrelat

fühlen mit - Dat

04

fülen nach-Dat

sich/Akk fühlen

sich/Akk fühlen

\section{führen}

führen

Pertinenzelement im Dativ
Po Die Familie frühstückt (gut).

Po Der Kranke fühlte große Schmerzen. Die Krankenschwester fühtt ihm den Puis.

INF-: $\quad$ Ich fühlte mein Herz schlagen.

DASS: Das Kind fuhlte <es>, daß seine Eltern sich gestritten hatten.

FRAG: Das Kind konnte <es> fühlen, ob seine Eltern sich gestritten hatten.

Po Die Eltern fülen mit der kranken Tochter.

PO Das Kind fühlt nach dem Schlüssel.

Po Der Junge fühtte sich als Mann.

Po Der Dieb fühtte sich schuldig.

P1 Ich führe den Haushalt.

Ich führe ein bequemes Leben.

Dieses Geschäft führt Lebensmittel.

Die.Mutter führt dem $A B C$-Schützen die Hand. 
führen zu-Dat

014

4:SE mit obligatorischem Korrelat

führen

015

führen

$01(6$

führen zu-Dat

04

\section{4:SE mit obligatorischem} Korrelat

führen

sich/Akk führen

*in Versuchung führen mit-Dat

*vor Augen führen

*mit sich führen

$\mathrm{T}$

\section{funktionieren}

funktionieren

funktionieren

O

\section{fürchten}

furchten
P1 Die Aussagen führten den Polizisten zu falschen Schlüssen.

INF+:

Diese Aussagen führten den Polizisten dazu, fali; :1!e Schlüsse zu ziehen.

'P1 Die ReisegeselZschaft führt Ausflüge im Programm.

P1 Der Hund führt den Blinden (über die Straße).

Po Die mangelnde Koordination hat zum. Zusammenbruch der ganzen Abteilung geführt.

DASS :

Sein Benehmen führte dazu, daß der Polizist falsche Schliusse zog.

PO Die Tür führt auf den Hof.

PO Der Häftling hat sich ordentlich geführt.

P1 Er hat ihn mit seinen Versprechungen in Versuchung geführt, seine Familie im Stich zu Zassen.

P1 Der Arzt führte ihm seinen Zustand vor Augen.

Po Ich führe [immer] einen Regenschirm mit mir.

PO Die Maschine funktioniert.

Po Der Wankelmotor funktioniert anders als der ottomotor.

P1 Die Studenten fürchten die Prüfungen. 


\section{$1: \mathrm{SE}$ ohne Korrelat}

fürchten für-Akk

furchten um-Akk

sich/Akk fürchten (vor-Dat)

4:SE mit fakultativem Korrelat

4:SE mit obligatorischem Korrelat
INF+:

DASS :

HPTS :

04

04

$0(4$

INF+:

DASS :
Der Student furchtet, durch die Prifung zu fallen. Der Student fürchtet, daß er durch die Prüfung fällt. Der Student fürchtet, er fällt durch die Prüfung.

P2 Er fürchtet für sein Leben.

P2 Die Hausfrau fürchtet um den Braten.

PO Die Kinder furchten sich (vor dem Gewitter). Die Kinder füchten sich <davor>, allein bleiben zu müssen.

Die Kinder fürchten sich davor, daß sie allein bleiben müssen. 
geben

geben

013

0:SE Zum Korrelat vgl. Einleitung 6.3.5.

geben auf - Akk

4:SE mit obligatorischem Korrelat

\section{BEMERKUNG :}

geben für - Akk

$4: \mathrm{SE}$ mit obligatorischem Korrelat

014

INF+ :

DASS :

014

INF+ :

DASS :

P1 Ich gebe nichts auf seine Meinung.

Ich gebe nichts darauf, seine Meinung zu hören.

Ich gebe nichts darauf, daß er pünktlich sein will. $E$, meist indefinit.

P1 Ich gebe mein Geld für eine Flasche Bier.

INF+: $\quad$ Der Vater hat sein Vermögen dafür gegeben, seinen Sohn zu retten.

DASS: $\quad$ Der Vater hat sein Vermögen dafür gegeben, daß sein Sohn gerettet wurde.

geben um - Akk

014

P1 Ich gäbe etwas um eine Fahrt nach Italien.

$4: S E$ mit obligatorischem Korrelat

\section{BEMERKUNG :}

geben (es)

geben (es)

\section{gebrauchen}

gebrauchen zu-Dat

gebrauchen
Ich gäbe etwas darum, noch einmal nach Italien fahren zu können.

$\mathrm{E}_{1}$ meist indefinit.

PO Es gibt vier Jahreszeiten.

PO In Rom gibt es viele Kirchen.

P1 Ich gebrouche den Bleistift aum Schreiben.
P1 Ergebraucht seine Müdigkeit als Entschuldigung. 


\section{gefallen}

gefallen

gefallen (es)

\section{gehen}

gehen

gehen

01

gehen mit - Dat

04

gehen um - Akk

04

4:SE mit obligatorischem

Korrelat

gehen

gehen

$0(5$

Pertinenzelement im Dativ gehen

gehen

gehen
PO Dein neues Kleid gefällt mir.

PO Es gefällt ihm [gut] in Mannheim.

Po Die Uhr geht.

[Heute] ging das Essen [in der Kantine] [wieder einmal].

Der Hefeteig geht.

P1 Die Jugend geht ihren Weg.

PO Volker geht [seit neuestem] mit Elke.

PO Das Gespräch geht wm aktuelle Probleme.

DASS: Das Gespräch geht darum, daß diese Reparaturwerkstatt schlecht ist.

FRAG: Das Gespräch geht darum, wie die neue Mode aussehen wird.

P2 Die Kinder gehen (auf der Straße).

PO Ergeht zur Bundeswehr.

Das Wasser ging ihm bis zum Knie.

P2 Der Mann geht (durch den Park).

PO Alles geht nach Wunsch.

P2 Der Fußgänger geht (sehr Langsam). 
gehen (es) um-Akk

4:SE mit obligatorischem Korrelat

INF+:

DASS :

FRAG :

gehen (es)

*seiner Wege gehen

*in die Brüche gehen

*in Erfuzzung gehen

*mit der Zeit gehen

*zu Bruch gehen

*zu Ende gehen

*zu Fuß gehen

*zur Hand gehen

*zur Neige gehen

\section{gehören}

gehören

gehören zu-Dat

03

04

4:SE mit obligatorischem Korrelat

gehören

INF+ :
PO Es geht (ihm) um Karls Kinder.

Es geht ihm darum, den Prozeß zu gewinnen.

Es geht ihm darum, daß er den Prozeß gewinnt.

Es geht ihm darum, ob er den Prozeß gewinnen kann.

po Dem Kranken geht es schlecht.

PO Der junge Mann geht seiner Wege.

po Ihre Freundschaft ging in die Brüche.

Po Seine Winsche gingen in Erfüllung.

Po Die alte Dame geht mit der Zeit.

PO Das notgelandete Flugzeug ging zu Bruch.

PO Die Arbeit geht zu Ende.

P2 Ich gehe zu Fuß in die stadt.

PO Die Tochter geht ihrer Mutter zur Hand.

PO Der Vorrat geht zur Neige.

Po Das Auto gehört meinem Vater.

PO Zum Fußballspiel gehören zweiundzwanzig Spieler. Viel Geschick gehört zur Lösung dieser Aufgabe. Viel Geschick gehört dazu, diese Aufgabe zu Zösen.

PO Fische gehören ins Wasser. 
sich/Akk gehören

O:SE Zum Korrelat vgl. Einleitung 6.3 .5 .

o

\section{INF+:}

DASS :

\section{BEMERKUNG :}

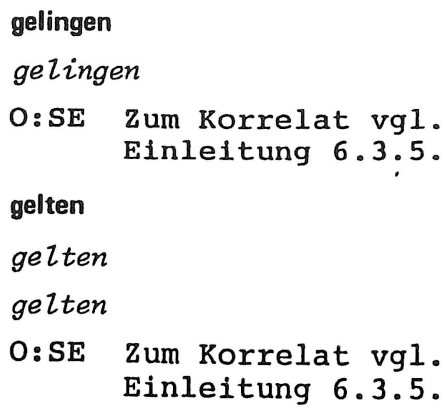

gelten bei - Dat

gezten für-Akk

gezten
PO Das gehört sich [nicht].

Es gehört sich nicht, von der Arbeit anderer Leute zu Zeben.

Es gehört sich nicht, daß man von der Arbeit anderer Leute lebt.

$E_{O}$ meist indefinit oder SE.

Po Das Essen ist (der Hausfrau) gelungen.

INF+: Der Hausfrau ist es gelungen, ein gutes Essen zu kochen.

PO Der Vertrag gizt.

Po Deine Liebe gilt mir viel.

INF+: $\quad$ Es gilt mir viel, morgens lange schlafen zu durfen.

DASS: $\quad$ Esgizt mir viel, daß du mich liebst. $\mathrm{E}_{1}$ meist indefinit.

PO Ordnung gilt bei Herm Schumacher sehr viel. $\mathrm{E}$, meist indefinit.

Po Ihre ganze Liebe gizt ihrer Katze.

PO Dieses Verkehrszeichen gilt für Fußgänger.

Po Fleißgilt als gute Eigenschaft. 
O:SE Zum Korrelat vgl. Einleitung 6.3 .5 .

gelten (es)

gelten (es)

geschehen

geschehen

geschehen mit-Dat

04

\section{BEMERKUNG :}

geschehen

\section{gewinnen}

gewinnen

gewinnen ous - Dat

014

gewinnen für - Akk

014

INF+ :

1

9

9: INF+:

(n)

4:SE mit obligatorischem Korrelat
Es gilt als gute Eigenschaft, fleißig zu sein.

PO Es gilt seinen Kopf.

PO

Es gizt zu gewinnen.

Po Dirwird nichts geschehen.

PO Mit dem neu eingelieferten Patienten ist noch nichts geschehen.

$E_{1}$ meist indefinit.

Po Das Unglück ist (um fünf Uhr) geschehen.

P1 Ich habe (tausend Mark) gewonnen.

[Natülich] hat der 1. FC Kaiserslautern [auf dem Betzenberg] (das Spiei gegen Braunschweig) gewonnen.

P1 Die Bouern gewinnen ous Milch Käse.

P1 Er hat sie für seinen Plan gewonnen.

INF+: $\quad$ Er gewann seine Kollegen dafür, seinen Plan au unterstützen.

DASS: $\quad$ Er gewann seine Kollegen dafür, daß sie seinen Plan unterstützen. 
gewinnen

017

gewinnen an - Dat

04

014

gewöhnen an - Akk

4:SE mit obligatorischem Korrelat

INF+ :

DASS :

\section{gießen}

gießen (es)

gießen

gießen

Pertinenzelement im Dativ

\section{glauben}

glauben

01

1:SE ohne Korrelat

BEMERKUNG :
P1 Der Chef hat ihn als neuen Mitarbeiter gewonnen.

P2 Das Mädchen gewinnt an Sicherheit.

P1 Die Mutter hat ihre Kinder an Pünktlichkeit gewöhnt.

Ich habe mich [schnell] an die neue Wohnung gewöhnt.

Die Mutter hat ihre Kinder daran gewöhnt, pünktlich zu sein.

Die Mutter hat ihre Kinder daran gewöhnt, daß sie püntlich sind.

PO Es gießt [in Strömen].

P1 Karingießt die Blumen.

P1 Der Kellner gießt Wein in das Glas.

Man gießt ihm Wasser über den Kopf.

P1 Ich glaube [nicht] alles.

Ich glaube, krank zu werden.

Ich glaube, daß ich krank werde.

Er glaubt, er sei krank.

$E_{1}$ meist indefinit oder SE. 


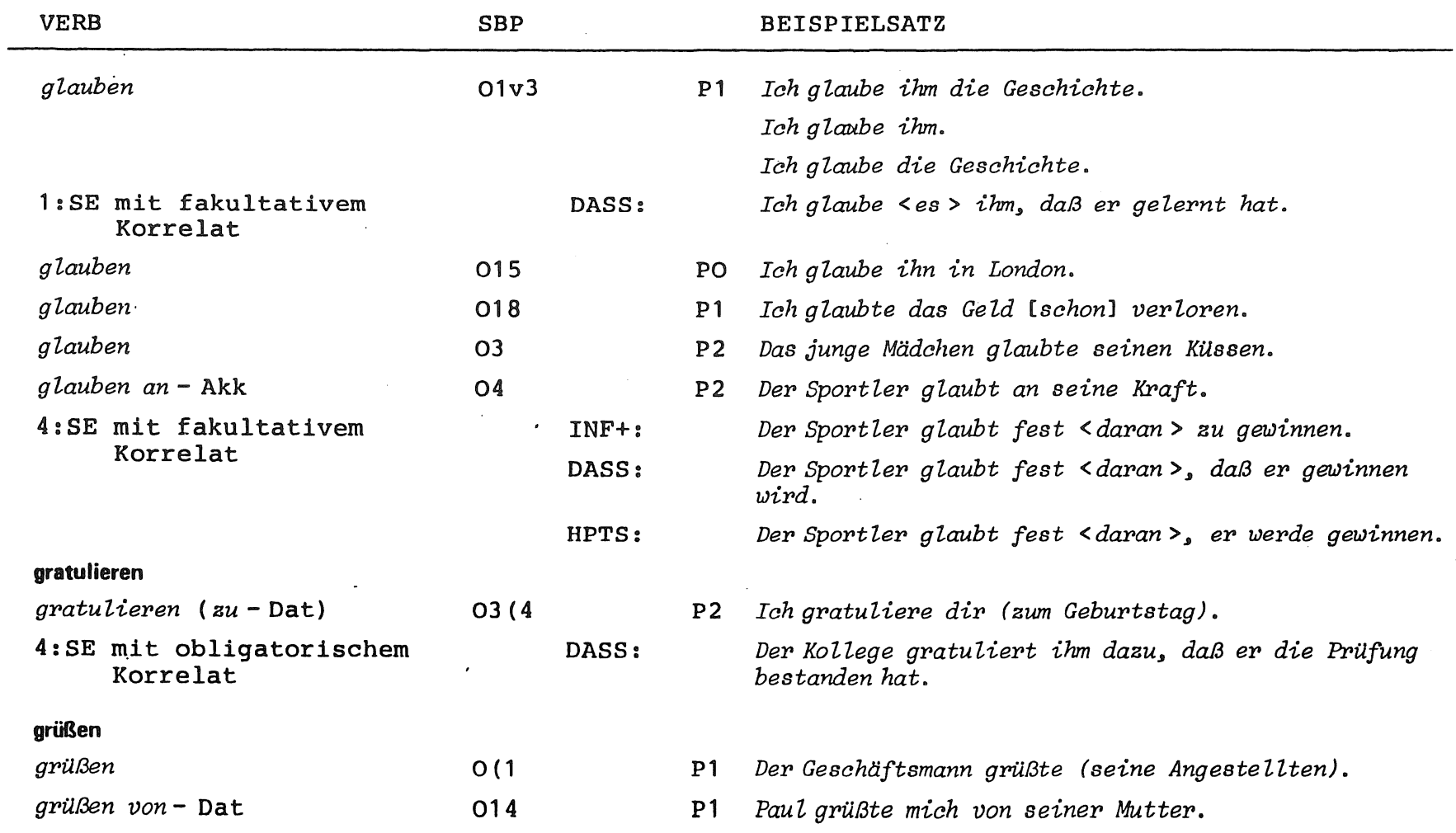




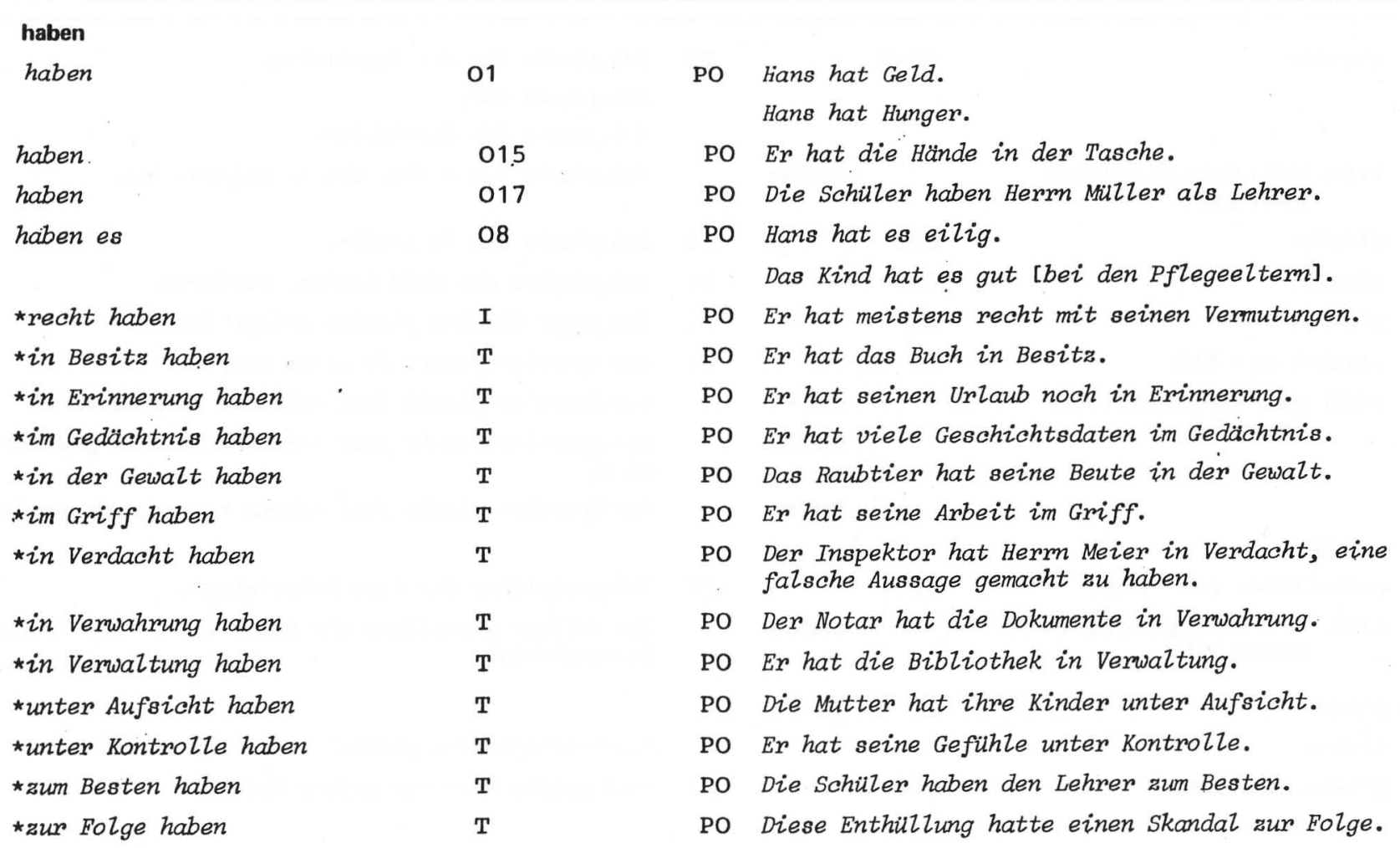


*zur Frau haben

*zum Gegenstand haben

*zur Hand haben

*zum Mann haben

*zur Seite haben

*zum Thema haben

*zu tun haben es mit-Dat

*zur Verfügung haben

*zum Vorbild haben

*zrom Ziel haben

\section{halten}

halten
PO Er hat eine ehemalige Schauspielerin zur Frau.

Po Das Gespräch hat den Umveltschutz zum Gegenstand.

Po Der Gesprächsteilnehmer hat viele Daten zur Hand.

PO Sie hat einen Amerikaner zum Mann.

PO Das Staatsoberhaupt hat immer Leibwächter zur Seite.

PO Das Buch hat den Sozialismus zum Thema.

PO Wir haben es hier mit einem schwierigen Problem zu tun.

PO Viele Professoren haben eine Bibliothek zur Verfügung.

Po Viele Jungen haben einen Sportler zum Vorbild.

Po Die Reform hat die Verbesserung der Sozialleistungen zum Ziel.

P1 Der Lehrer hält [in der ersten Klasse] Unterricht. Sein Freund hält Tiere.

Ilona Gusenbauer hielt den Weltrekord [im Hochsprung].

halten

Das Orchester hält den Takt.

Der Angestellte hält Ordnung [auf seinem Schreibtisch].

Er hält sich den Kopf. 


\section{halten}

halten fü - Akk

1:SE Zum Korrelat vgl. Einleitung 6.3 .5 .

014

0113

014

INF+:

DASS :

FRAG :

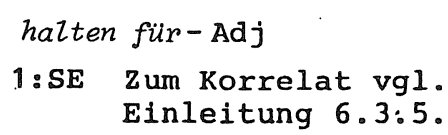

1:SE Zum Korrelat vgl. Einleitung 6.3:5.

DASS :
P1 Er hält (seiner Freundin) das Versprechen.

P1 Er hält ihn für den Dieb.

Ich halte es für einen Vorteil, fremde Sprachen zu verstehen.

Ich halte es für einen Vorteil, daß wir englisch sprechen können.

Ich halte es für ein Problem, ob wir genug Zeit haben werden.

P1 Die Ärzte halten den Mann für tot.

INF+: $\quad$ Ich halte es für wichtig, fremde Sprachen zu verstehen.

DASS: $\quad$ Ich halte es für wichtig, daß man fremde Sprachen lernt.

FRAG: $\quad$ Ich halte es für fraglich, ob er kommt.

P1 Meine Schwester hält nichts von der neuen Mode.

INF+: Meine Schwester hält nichts davon, lange Röcke zu tragen.

Meine Schwester hält nichts davon, daß sie lange Röcke tragen solz.

$E$, meist indefinit.

P1 Die Eltern halten das Kind im Bett.

P1 Er hält ein Buch (in der Hand).

P1 Er hält die Hand vor den Mund. 


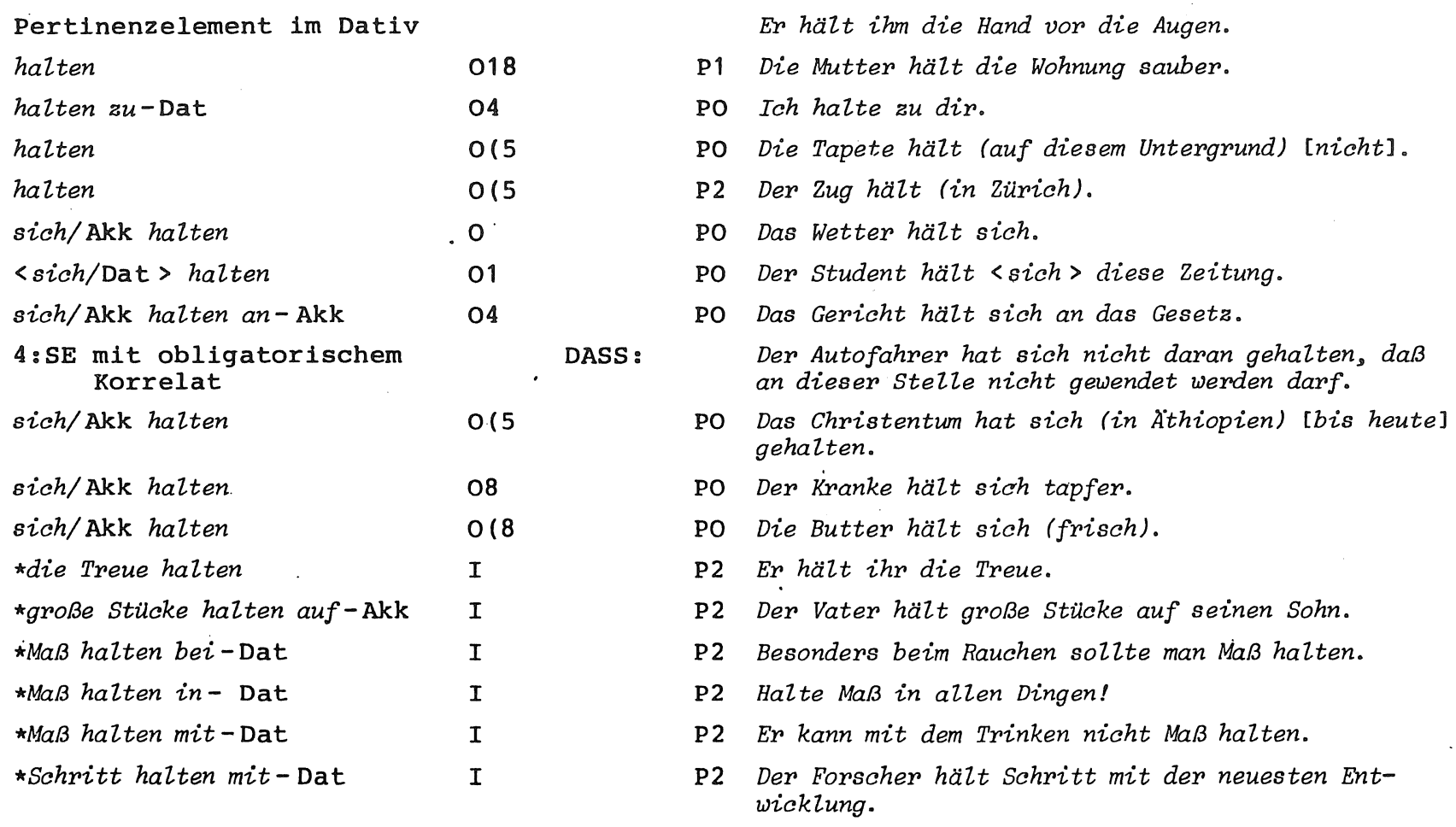


*Wort halten mit-Dat

*in Atem halten

\section{handeln}

hande $2 n$

o

handeln

01

handeln mit-Dat

04

handeln von-Dat

04

4:SE mit obligatorischem Korrelat

handeln mit-Dat um-Akk

4:SE mit obligatorischem Korrelat

handeln ( an - Dat)

sich/Akk handeln (es) um - Akk
P2 Er hielt stets Wort mit dem, was er versprach.

P1 Das Spiel hielt die Zuschouer in Atem.

P2 [In dieser Angelegenheit] hat die Regierung [nicht rechtzeitig ] gehandeit.

P1 [An der Börse] wurden Bezugsrechte von den Banken [zu einem niedrigen Kurs] gehandelt.

P2 Hans handelt mit Schokolade.

Po Das Buch handelt vom Leben nach dem Tode.

DASS :

FRAG:

$04 v 4$

P2 Der Kaufmann handelt mit dem Geschäftspartner um einen niedrigen Preis.

Der Kaufmann handelt mit seinem Geschäftspartner. Der Geschäftspartner handelt um einen niedrigen Preis.

DASS: $\quad$ Der Geschäftsmann handelt mit seinem Partner darum, daß die Ware billiger wird.

P2 Er hat schlecht (an mir) gehandelt.

Po Es handelt sich [bei diesem Preis] um ein günstiges Angebot. 


\section{4:SE mit obligatorischem Korrelat}

INF+ :

DASS :

FRAG :

\section{hängen}

höngen an - Dat

04

hängen

hängen

Pertinenzelement im Dativ

hängen

BEMERKUNG :

\section{hängen}

hängen

nöngen

hängen

BEMERKUNG :

\section{heben}

heben

heben
01

016

05

06

018

01

016
Es handelt sich darum, das Motorrad möglichst teuer zu verkaufen.

Es handelt sich darum, daß die Klingel repariert werden muß.

Es handelt sich darum, ob genug Plätze reserviert werden können.

PO Das Kind hängt an seinen Eltern.

PO Das Bild hängt über dem Schreibtisch.

PO Die Haare hängen ihr ins Gesicht.

PO Der Baum hängt volzer Bimen.

STAMMFORMEN : hängen, hing, hat gehangen.

P1 Der Henker hängte den Verbrecher.

P1 Die Hausfrau hängt die Bluse auf den Kleiderbügel.

P1 Sie hängt das Bild gerade.

STAMMFORMEN : hängen, hängte, hat gehängt.

P1 Der Archäologe hat einen Schatz gehoben.

P1 Der Vater hebt das Kind aus dem Bett. 


\begin{tabular}{|c|c|c|}
\hline heben & $01(6$ & \\
\hline sich/Akk heben & 0 & \\
\hline \multicolumn{3}{|l|}{ heiraten } \\
\hline heiraten & $O(1$ & \\
\hline \multicolumn{3}{|l|}{ heißen } \\
\hline heiBen , & 011 & \\
\hline \multirow[t]{2}{*}{$1:$ SE ohne Korrelat } & & INF-: \\
\hline & & INF+: \\
\hline \multicolumn{3}{|l|}{ BEMERKUNG : } \\
\hline heißen & 017 & \\
\hline heißen nach - Dat & 04 & \\
\hline heißen & 07 & \\
\hline \multirow[t]{2}{*}{ heiBen (es) } & 9 & \\
\hline & $9:$ & $\begin{array}{l}\text { DASS: } \\
\text { HPTS : }\end{array}$ \\
\hline \multirow[t]{3}{*}{ heiBen (es) } & 9 & \\
\hline & $9:$ & INF-: \\
\hline & & INF+: \\
\hline
\end{tabular}

P1 Die Arbeiter heben die Kiste (auf den Wagen).

po Der Boden hebt sich.

P1 Hans heiratet (seine Freundin).

P1 Ich heiße dich etwas.

Ich heiBe dich das tun.

Ich heiBe dich, das zu tun.

Unpersönliche $E_{1}$ meist indefinit oder SE:

P1 Der Mann heiBt Hans einen Dummkopf.

PO Die Straße heißt nach Theodor Heuss.

po Diese Straße heißt Bonhoefferstraße.

PO

Es heißt, daß dicke Menschen gemütiich sind. Es heißt, dicke Menschen sind gemützich.

PO

Jetzt heißt es, die Sache anders anfassen.

Jetat heißt es, die Sache anders anzufassen.

heizen

heizen

P1 Mein Nachbar heizt (seinen Ofen). 
heizen (mit-Dat)

heizen

$0(4$

018

03

\section{helfen}

helfen

helfen gegen - Akk

$0(34$

O:SE Zum Korrelat vgl. Einleitung 6.3.5.

helfen bei-Dat

O3v 4

INF+:

O:SE Zum Korrelat vgl. Einleitung 6.3.5.

\section{4:SE mit fakultativem} Korrelat

\section{herstellen}

hersteilen

herstellen (aus-Dat)

\section{hindern}

hindern an - Dat

INF+:

DASS :

INF+:

01

$01(4$

014
P2 Mein Nachbar heizt (mit Kohle).

PO Holz heizt (gut).

P2 Der Rettungsschwimmer hilft dem Ertrinkenden.

PO Das Feuer hilft (dir) gegen die Kälte.

Diese Tabletten helfen (mir) [meistens] gegen Kopfschmerzen.

Es hilft gegen Grippe, heiße Milch zu trinken.

P2 Der Ehemann hilft seiner Frau beim Kochen. Der Ehemann hilft seiner Frau.

Der Ehemann hilft beim Kochen.

P1 Das Amt stellt die Telefonverbindung her.

P1 Die Fabrik stellt (aus Holz) Spielzeug her. Es hat mir sehr geholfen, einige Stunden lang Ruhe zu haben.

Es hilft mir bei der Arbeit, daß du mich berätst. Der Ehemann hilft seiner Frau 〈dabei>, das Essen zu kochen.

P1 Der Hund hinderte den Dieb om Stehlen. 


\section{4:SE mit fakultativem Korrelat \\ 4:SE mit obligatorischem Korrelat}

hindern bei - Dat

$01 v 4$

\section{holfen}

hoffen

1:SE ohne Korrelat.

01

INF+:

DASS :

HPTS :

\section{BEMERKUNG :}

hoffen auf - Akk

4:SE mit fakultativem Korrelat

INF+ :

DASS :

04

INF+ :
P1 Dieses Kleid hindert sie bei der Arbeit.

Das Kleid hindert sie.

Das Kleid hindert bei der Arbeit.

P1 Wir hoffen das Beste.

Ich hoffe, bald einen Brief von dir zu bekommen. Ich hoffe, daß du mir häufig schreibst. Ich hoffe, du schreibst mir wieder. $\mathrm{E}$, meist indefinit oder SE.

DASS :

HPTS :

P2 Der Dieb hofft auf seinen Rechtsanwalt. Der Schwerkranke hofft <darauf >, wieder gesund zu werden.

Mein Vater hofft 〈darauf>, daß er die neue Stelle erhält.

Der Patient hofft <darauf>, er werde bald entlassen.

PO En holt Atem

Er holt [tief]. Luft. 


\section{holen}

$01(6$

sich/Dat holen

01

\section{hören}

hören

1:SE ohne Korrelat

1:SE mit fakultativem Korrelat

01

INF- :

DASS :

FRAG:

hören (von-Dat)

$1: \mathrm{SE}$ ohne Korrelat

1:SE mit fakultativem Korrelat

\section{HPTS:}

DASS :

FRAG:

hören auf - Akk

4:SE mit obligatorischem Korxelat

DASS :

FRAG :

hören von - Dat
P1 Der Student hat das Buch (aus der Bibliothek) geholt.

po Ich habe mir einen schlimmen Schnupfen geholt.

P1 Der Hund hört den Mann. Ich höre den Monn konmen.

Ich habe <es > genau gehört, daß jemand komnt. Ich habe <es > genau gehort, wie ihr euch gestritten habt.

P1 Ich habe diese Geschichte (von einem Studenten) gehort. Der Mann hörte von seiner Frau, es sei etwas Schiimmes passiert.

Ich habe <es> von Vater gehort, daß heute Besuch kormit.

Ich habe <es> von meiner Mutter gehort, wann unsere Tante ankommt.

P2 Der Hund hort ouf seinen Herrn.

Der Junge hörte nicht darauf, daß seine Freunde ihn zurückriefen.

Der Junge horte nicht darauf, wie seine Freunde ihn zurückriefen.

P2 Tch habe von dieser Geschichte gehört. 


\section{4:SE mit fakultatiyem Korrelat}

hupen

husten

husten

husten

*etwas husten
0

\section{DASS :}

FRAG:

HPTS :

01

I
Ich habe schon <davon> gehört, daß bei euch eingebrochen wrirde.

Man hat noch nichts <davon> gehört, ob die Passagiere den Absturz überlebt haben.

Ich habe schon <davon> gehört, du solzst krank gewesen sein.

PO Der Großvater hört schlecht.

P2 Der Autofahrer hupt.

P2 Ich huste.

P1 Der Kranke hustet Blut.

PO Ich huste dir etwas. 


\section{informieren}

informieren (uber - Akk)

0114

4: SE mit fakultativem Korrelat

\section{interessieren}

interessieren

O:SE Zum Korrelat vgl. Einleitung 6.3 .5 .

interessieren für - Akk

sich/Akk interessieren fïr - Akk Korrelat

01

INF+: DASS : FRAG :

DASS :

FRAG :

HPTS:

$$
\text { 4:SE mit obligatorischem }
$$$$
\text { Korrelat }
$$

INF+:
P1 Mein Freund informiert mich (über seine schlinme Lage). Mein Freund hat mich <dariber > informiert, daß er sich in einer schlimmen Lage befindet.

Mein Freund hat mich <dariber > informiert, wie schwierig die Lage für ihn ist.

Mein Freund hat mich <dariber > informiert, er befinde sich in einer schwierigen Lage.

P1 Dein Verhalten interessiert mich.

Es interessiert mich, das neue Stück zu sehen.

Es interessierte mich; daß du in die Stadt gezogen bist.

Es interessiert mich, warum du das zu mir gesagt hast.

Po Für diese Arbeit wirst du niemand interessieren können.

PO Der Mann interessiert sich für mich. Ich interessiere mich dafür, diese Kirche zu besichtigen.

DASS: Die Polizei hat sich dafur interessiert, daß der Untermieter von Frau Müller ausgezogen ist.

FRAG: Der Gast interessiert sich dafür, was im Theater gespielt wird. 


\section{irren}

<sich/Akk > irren (in - Dat) O(4

4:SE mit obligatorischem Korrelat

BEMERKUNG :

irren

irren

BEMERKUNG :
PO Du irrst <dich> (in deiner Annahme).

Du hast dich darin geirrt, daß du heute kommen solltest.

STAMMFORMEN: irren, irrte, hat geirrt.

Po Sie irren durch den Wald.

STAMMFORMEN: irren, irrte, ist geirrt. 


\section{kämmen}

köronen

01

Pertinenzelement im Dattv

kömmien

Pertinenzelement im Dativ

\section{kaufen}

kaufen

kaufen nit - Dat

01

014

\section{4:SE mit obligatorischem Korrelat}

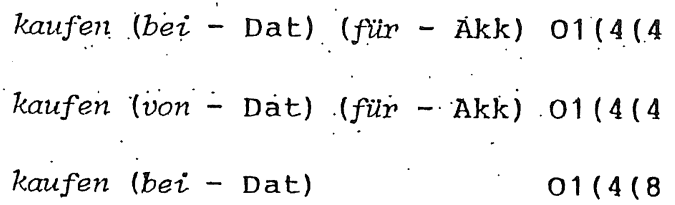

$01(418$
P1 Marlies kämnt ihrem Bruder die Haare. Das Mädchen könmt sich die Haare.

P1 Die Mutter hat dem Kind die Haare ins Gesicht gekömmt. Der Junge hat sich die Haare ins Gesicht gekönmt.

P1 Viele Leute koufen das neue Buch.

P1 Dex verdächtigte. Finanzmakler hatte den Untersuchungsrichter mit einer sechsstelligen Summe gekauft.

- DASS: Der verdächtigte Finanmakler hatte den Untersuchungsrichter damit gekauft, daß er ihm eine sechsstellige Summe versprach.

P1 Er hät das Auto (beim Gebrauchtwagenhändler) (für DM 1000, -) gekauft.

P1 Er. hat den Rasenmäher (von seinem Freund) (für DM.200, -) gekauft.

P1 Er hat das Auto (beim GebrauchtwagenhändZer) (bilzig) gekauft.

Er hat das Auto (beim Gebrauchtwagenhändlex) (auf liaten) gekauft.

P1 Er hat den Zweitwagen (von seinem Freund) (billig) gekauft. 


\section{kennen}

kennen

kennen

kennen

\section{kennenlernen}

kennenlernen

kennenlernen

\section{klappen}

kZappen

klappen

klappen (es) (mit-Dat)

4:SE mit fakultativem Korrelat

\section{kleben}

kleben

01

kleben

016

Pertinenzelement im Dativ kZeben

Pertinenzelement im Dativ

01

01

0

016

$(4$
017

018

\section{DASS :}

Po Die Sache klappt.

P1 Er klappt den Autositz nach vorne.

PO Es klappt (mit der Wohnung).

Es klappt <damit>, daß wir die Wohnung bekommen.

P1 Dieser Klebstoff klebt auch Kunststoff.

P1 Der Student klebt Bilder an die Wand. Er klebt ihm das Pflaster auf die Wunde.

po Die Briefmarke klebt (auf dem Umschlag). Das Hemd klebt ihm am Leibe. 


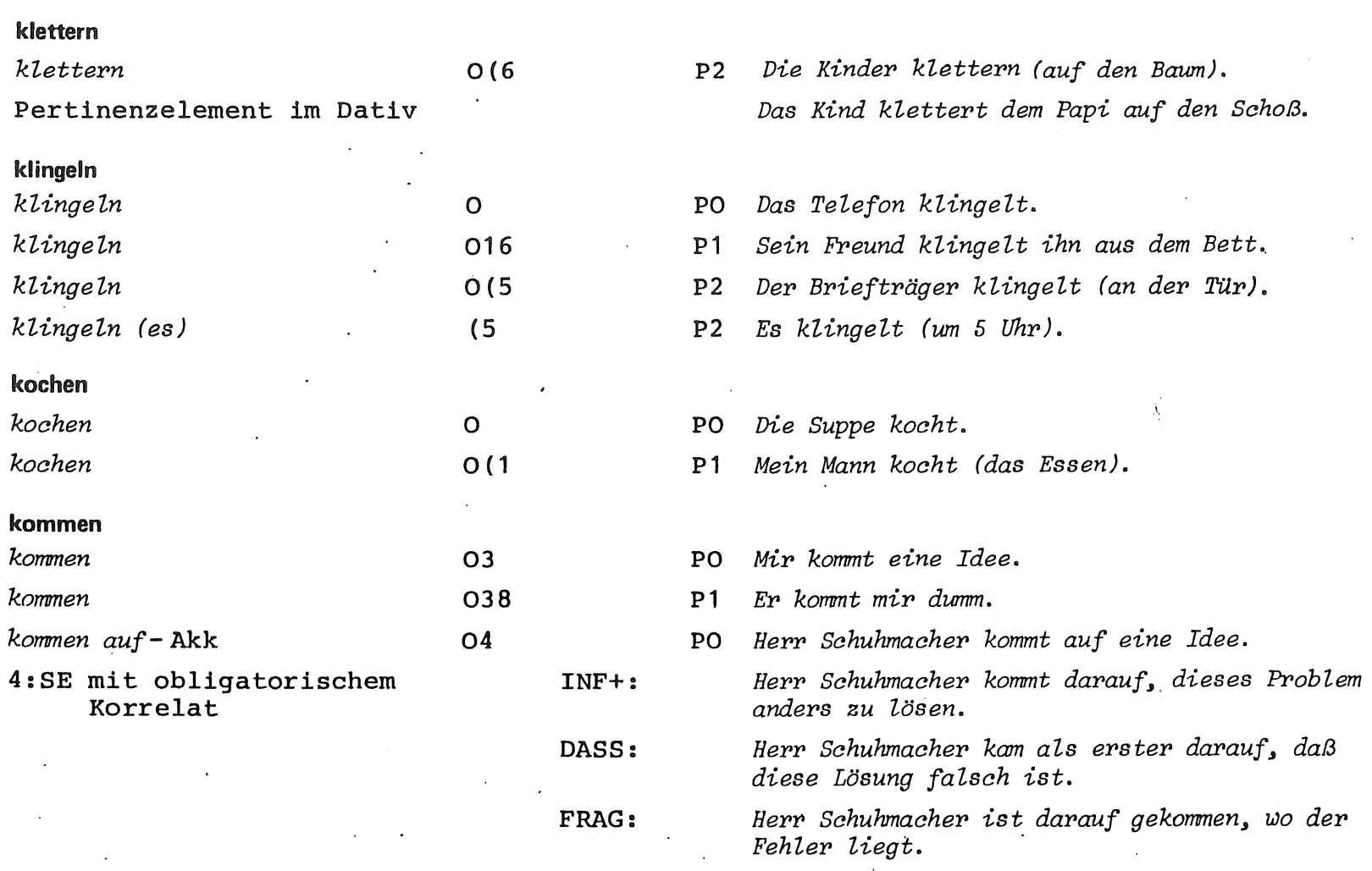




\section{kommen um - Akk \\ kommen von-Dat \\ 4:SE mit obligatorischem Korrelat \\ kommen zu-Dat \\ 4:SE mit obligatorischem Korrelat}

kommen

kommen

*aus der Mode kommen

*aus dem Takt kommen

*aus dem Tritt kommen

*aus der Ubung kommen

*außer Gebrauch kommen

*in Anwendung kommen

*in Bedrängnis kommen

*in Berührung kommen mit-Dat

*in Betracht kommen

*in Bewegung kommen
04

04

04

06

016

I

I

I

I

I

I

I

I

I
PO Emma kommt um den verdienten Erfolg.

PO Deine Krankheit kommt vom Rauchen.

DASS: . Deine Krankheit kommt davon, daß du zuviel geraucht hast.

PO Die Mutter kommt [kaum] zum Ausmhen.

INF+: Meine Mutter kommt kaum dazu, sich auszuruhen.

PO Die Bücher kommen in den Schrank.

PO Der Vater kommt (aus dem Büro).

PO Der Minirock kommt aus der Mode.

Po Die Cellistin kommt aus dem Takt.

Po Die Marschkolonne kommt aus dem Tritt.

PO Der Sportler ist aus der Ubung gekommen.

PO Der Name ist außer Gebrauch gekommen.

PO Das Gesetz kommt in Anwendung.

PO Durch seinen Leichtsinn kam er in große Bedrängnis.

PO Bei seinem Beruf kommt er mit vielen Menschen in Berührung.

Po Eine Steuersenkung kommt für die Regierung nicht in Betracht.

PO Die Menschenmenge kommt in Bewegung. 


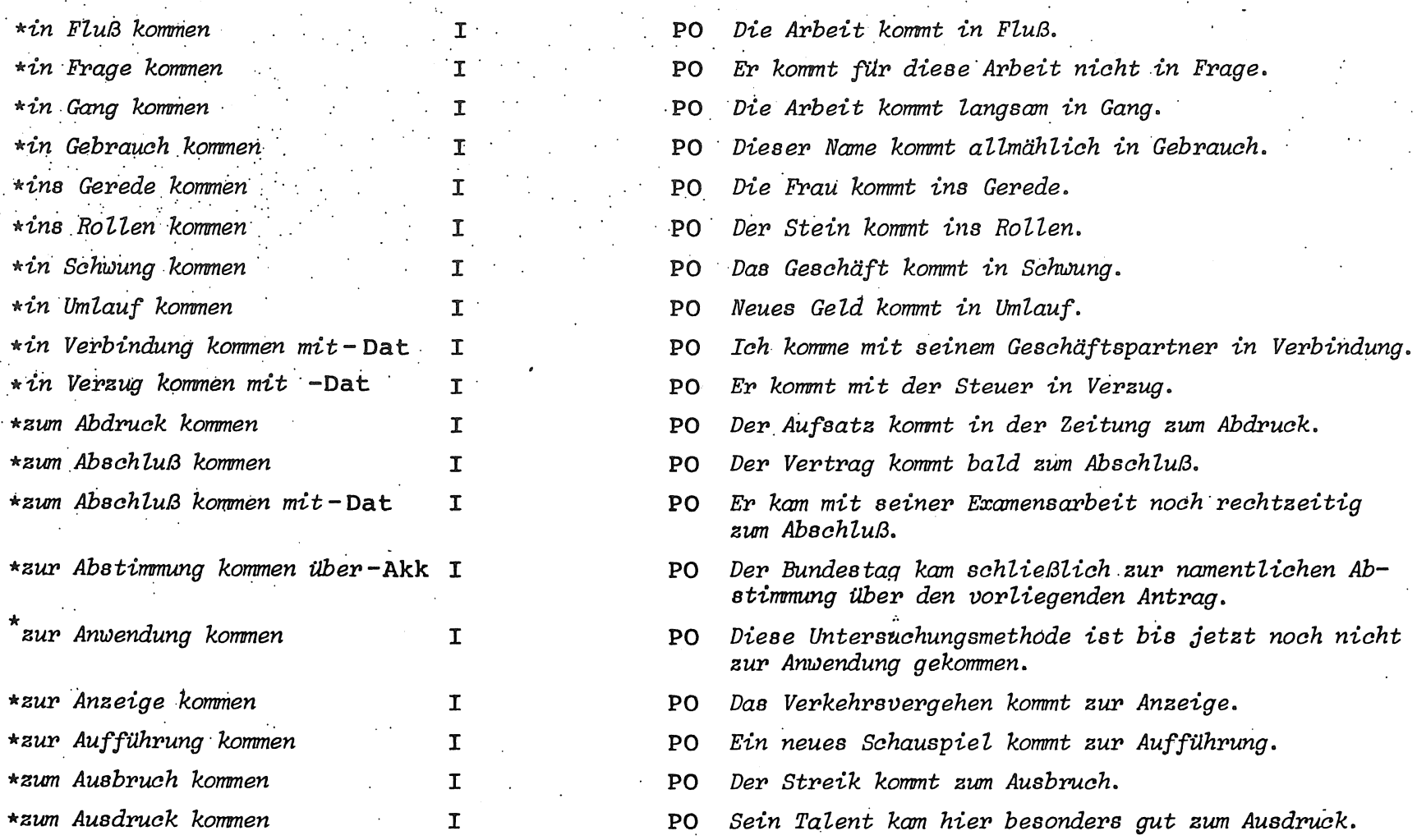


*zur Ausführung kommen

* zum Bewußtsein kommen

*zur Diskussion kommen

*zur Durchführung kommen

*zum Einsat: kommen

*zur Entscheidung kommen

*zur Erörterung kommen

*zu Fall kommen

*zu Gehör kommen

*zur Geltung kommen

*zur Kenntnis kommen

*zur Ruhe kommen

*zur Sprache kommen

«zum Stillstand kommen

*zum Verkauf kommen

*zur Verlesung kommen

*zur Versteigerung kommen

*zur Verteilung kommen

*zur Vorlage kommen

*zum Vorschein kommen
PO Dieser Plan kam nie zur Ausfuhrung.

PO Seine Intoleranz ist. ihm nie zum Bewribtsein gekommen.

PO Der neue Plan kormt morgen zur Diskussion.

PO Der Vorschlag des Betriebsrates kommt aur Durchführung.

PO Die neuen Maschinen kommen zum Einsatz.

po Die Sache kommt zur Entscheidung.

PO Der Vorfall kommt zur Erörterung.

po Die Gesetzesvorlage kommt heute zu Fall.

PO Diese Geschichte ist mir heute zu Gehor gekommen.

PO Seine Meinung kommt immer zur Geltung.

PO Der Vorfall ist mir noch nicht zur Kenntnis gekommen.

Po Die Menge kam zur Ruhe.

Po Der Vorfall wird morgen zur Sprache kommen.

PO Die Bewegung kommt zum Stillstand.

PO Die Ware kommt aum Verkauf.

Po Das Urteil kommt zur Verlesung.

PO Der kostbare Familienbesitz kommt zur Versteigerung.

Po Die Beute kam zur Verteilung.

PO Die Akten kommen zur Vorlage.

po Die Sonne komnt aum Vorschein. 
* zur Welt kommen

*zur Wirkung kommen

I

«zum Zug kommen mit-Dat

I

I

\section{korrigieren}

korrigieren

01

\section{kosten}

kosten

1:SE ohne Korrelat

kosten

O:SE Zum Korrelat vgl. Einleitung 6.3 .5 .

\section{kriegen}

kriegen

kriegen (von-Dat)

kriegen

kriegen

kriegen

*zur Frau kriegen
01

$01(4$

016

017

018

01

$0(11$

FRAG:

INE+

$\mathbf{T}$
PO Unser Sohn ist am ersten Mai zur Welt gekommen.

PO Das Kleid kommt gut zur Wirkung.

PO Er kommt mit seiner Taktik zum Zug.

P1 Das Flugzeug korrigiert seinen Kurs. Der Lehrer korrigiert die Hefte.

P1 Der Gast kostet das Fleisch. Die Hausfrau kostet, ob das Fleisch gut ist.

PO Das Kleid hat (mich) viel Geld gekostet. Es hat mich viel Mühe gekostet, eine Wohnung zu finden.

PO Ich kriege Schnupfen.

Seine Freundin kriegt ein Kind.

Po Hans kriegt (von Franz) Geld.

Po Hans kriegt die Leiter [nicht] durch die Tür.

Po Er kriegt Herrn Meier als Vorgesetzten.

PO Ulla kriegt das Holz [nicht] klein.

PO Er kriegt eine Verkäuferin aur Frau. 
*zum Mann kriegen

*zu tun kriegen es mit-Dat

\section{kümmern}

kümiern

O:SE Zum Korrelat vgl. Einleitung 6.3.5.

01

DASS :

FRAG :

sich/Akk kümmern um-Akk

04

4:SE mit obligatorischem Korrelat

kündigen

0

kündigen

$O(1) 3$

küssen

küssen

Pertinenzelement im Dativ küssen

INF+:

DASS :

FRAG :
Po Sie kriegt einen Angesteizten zum Mann.

PO Er kriegt es mit der Angst zu tun.

Po Die Krankheit kümmerte ihn [wenig]. Es kümmert die Eltern wenig, daß ihre Tochter immer spät nach Hause kommt.

Es kümmert die Eltern wenig, wann die Tochter nach Hause kommt.

po Die Großmutter kümmert sich um die Blumen.

P2 Der Mitarbeiter hat gekündigt.

P1 Die Firma kündigt (ihrem Mitarbeiter) (den Vertrag).

P1 Cäsar küßt seine Freundin.

Cäsar küßt seiner Freundin die Hand.

P1 Cäsar küßt seine Freundin auf die Stirn. Kurt küßt Gudrun auf die Wange. 


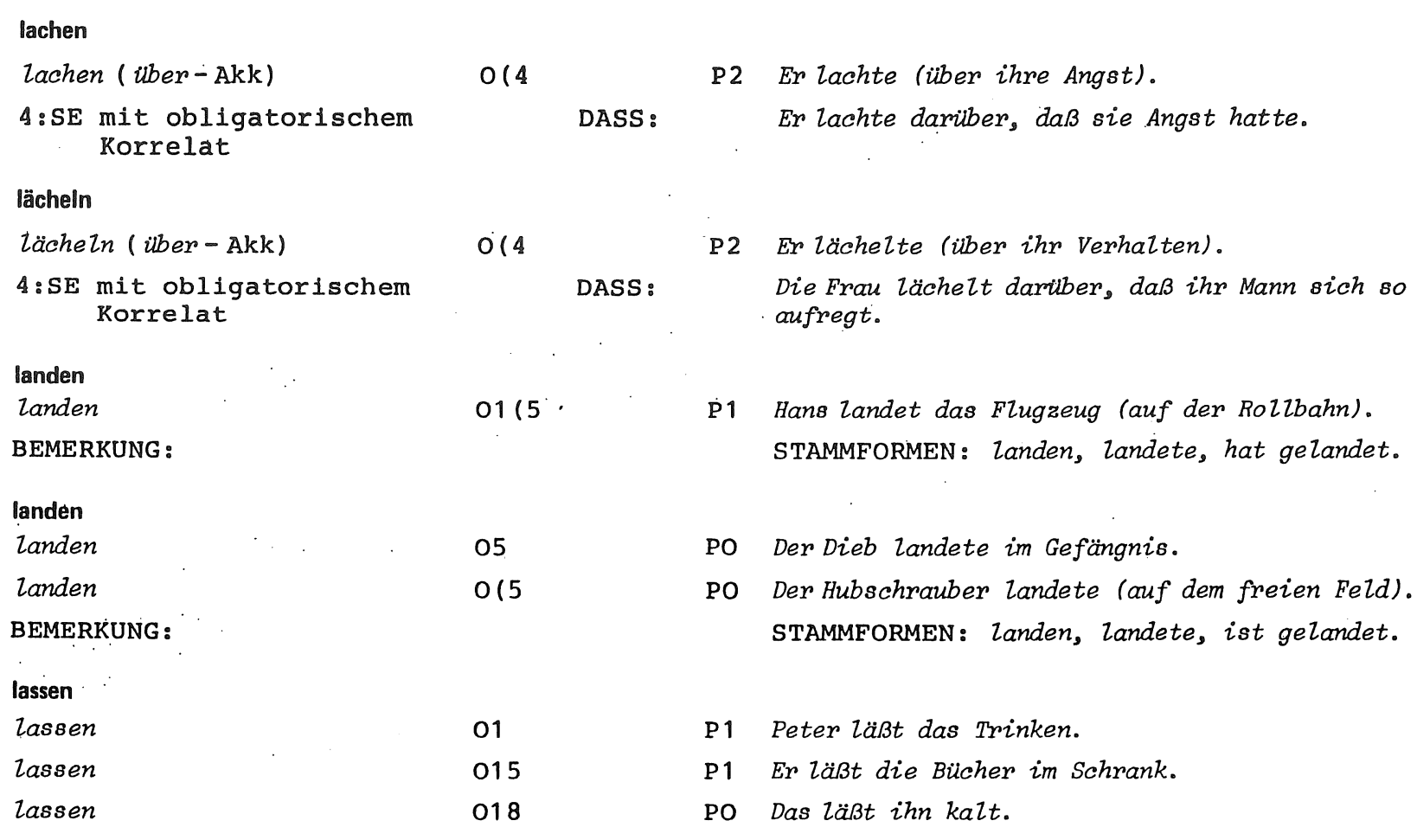




\begin{tabular}{|c|c|c|c|c|}
\hline \multirow[t]{2}{*}{ Zassen } & 09 & & PO & \\
\hline & $9:$ & INF-: & & Er Zäßt dich grüßen. \\
\hline bleibenlassen & 01 & & PO & Er Zäßt das Trinken bleiben. \\
\hline sich/Akk gehenlassen & 0 & & PO & Manfred läßt sich gehen. \\
\hline seinlassen & 01 & & PO & AZmut Zäßt das Rauchen sein. \\
\hline *am Leben Zassen & $\mathrm{T}$ & & P1 & Der Junge Zieß die Ameise am Leben. \\
\hline *außer acht lassen & $\mathrm{T}$ & & P1 & Der Bergsteiger ließ die Warnung außer acht. \\
\hline *in Frieden Zassen & $\mathrm{T}$ & & P1 & $\begin{array}{l}\text { Hans und Fridolin Zassen den Homster Urmel in } \\
\text { Frieden. }\end{array}$ \\
\hline «in Ruhe Zassen & $\mathrm{T}$ & & P1 & Karl-Gustav läßt seine Frau in Ruhe. \\
\hline *im ungewissen Zassen über - Akk & $\mathbf{T}$ & & $\mathrm{P} 1$ & $\begin{array}{l}\text { Der Lehrer ließ den Schüler über seine Noten im } \\
\text { ungewissen. }\end{array}$ \\
\hline 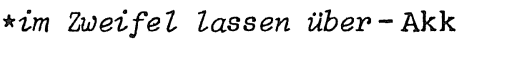 & $\mathrm{T}$ & & P1 & $\begin{array}{l}\text { Der Arzt ließ den Patienten über seine Krankheit } \\
\text { im Zweifel. }\end{array}$ \\
\hline \multicolumn{5}{|l|}{ laufen } \\
\hline Zaufen & 0 & & PO & $\begin{array}{l}\text { Der Motor Zäuft. } \\
\text { Die Arbeit Zäuft [wie von selbst]. }\end{array}$ \\
\hline Pertinenzelement im Dativ & & & & Dem Kind läuft die Nase. \\
\hline Zaufen & 01 & & P1 & Der Sportzer läuft eine gute Zeit. \\
\hline Zaufen unter-Dat & 04 & & $\mathrm{PO}$ & Ihr Konto läuft unter der Nummer 2177. \\
\hline Zaufen & $0(5$ & & $\mathrm{PO}$ & Ein neuer Film läuft (im Kino). \\
\hline Zaufen & 06 & & $\mathrm{PO}$ & Er läuft in sein Unglück. \\
\hline
\end{tabular}




\section{Pertinenzelement im Dativ}

Zoufen

sich/Akk Zaufen

*Gefahr Zaufen

\author{
leben \\ Zeben \\ Zeben für - Akk \\ $4: \mathrm{SE}$ mit obligatorischem \\ Korrelat
}

Zeben von - Dat

4:SE mit obligatorischem Korrelat

Zeben

05

Zeben

legen

Zegen
Er läuft mir über den Weg.

Die Tränen Zaufen ihr übers Gesicht.

Po Das Kind ist (aus dem Haus) gelaufen.

Po Er hat sich müde gezaufen.

PO Er läuft Gefahr, sich anzustecken.

PO Der Verunglückte Zebt.

P2 Der Forscher lebt für seine Wissenschaft. Der Arzt lebt nur dafür, den Kranken zu helfen.

Herr Maier lebt nur dafïr, daß seine Kinder studieren können.

P2 Mein Onkel lebt von seiner Rente.

INF+: . Er lebt in erster linie davon, Bücher zu verkaufen.

DASS: $\quad$ Er lebt davon, daß seine Frau Gemüse verkauft.

P2 Die Familie lebt auf dem Land.

P2. Die Familie lebt gut.

P1 Vögel Zegen Eier.

Die Wahrsagerin legt Karter.

Er hat Feuer gelegt. 


\section{Zegen}

016

Pertinenzelement im Dativ

sich/Akk Zegen

0

*den Akzent Zegen auf - Akk

*Gewicht Zegen auf - Akk

*Hand Zegen an-Akk

*Nachdruck legen auf-Akk

*Wert Zegen auf - Akk

*ans Herz legen

I

I

I

I

I

$\mathrm{T}$

\section{leiden}

Zeiden

1:SE mit fakultativem Korrelat

Zeiden

O 1

Zeiden

Zeiden an - Dat

Zeiden unter - Dat

4:SE mit obligatorischem Korrelat

01
P1 Der Schuller legt das Buch auf den Schrank.

Ler Polizist legt dem Dieb die Hand auf die Schulter.

PO Der Sturm hat sich gelegt.

P1 Er legt den Akzent ouf seine frühere Aussage.

P1 Der Bundeskanzler hat auf dieses Problem besonderes Gewicht gelegt.

PO Er legt Hand an sich.

PO Auf dieses Problem lege ich besonderen Nachdruck.

P1 Er legt Wert auf deine Anwesenheit.

P1 Die Mutter legt den Großeltern die Sorge fü ihre Kinder ans Herz.

PO Meine Arbeit leidet keinen Aufschub.

DASS: $\quad$ Ich leide <es > nicht, daß der Hund im Zimmer schläft.

PO Der Verletate leidet (Schmerzen).

P1 Ich Zeide keine Hunde im Haus.

PO Ich Zeide an Kopfschmerzen.

Po Der Hund leidet unter seinem Herrn.

INF+ : Das Kind litt darunter, immer allein zu sein.

DASS :
Das Kind litt darunter, daß es immer allein war. 


\section{leihen}

Zeihen

013

sich/Dat.Zeihen (von - Dat)

$01(4$

\section{lernen}

Zernen (ous - Dat)

1:SE mit fakultativem Korrelat

O 114

INF+:

DASS :

- FRAG :

HPTS : 4:SE mit obligatorischem
Korrelat

\section{BEMERKUNG :}

Zernen ( von - Dat)

1 :SE ohne Korrelat

1:SE mit fakultativem Korrelat

DASS :

$O(1) 4$
P1 Hugo lieh seiner Schwester zehn Mark.

Po Norbert hat sich zehn Mark (von Peter) geliehen.

P1 Ich habe (viel) (aus meinen Fehiern) gelernt. Ich habe <es> aus meinen Fehlern gelernt, vorsichtig zu sein.

Ich habe 〈es > aus meinen Fehlern gelernt, daß man vorsichtig sein sollte.

Ich habe <es> aus meinen Fehlern gelernt, wie man sich richtig verhalten muß.

Ich habe <es> aus meinen Fehlerm gelernt, ich muß mich anders verhalten. Peter hat nichts daraus gelermt, daß er schon drei Unfalle hatte.

$\mathrm{E}$, meist indefinit oder SE.

P1 Peter lernt (das Sparen) (von seiner Mutter).

INF-: $\quad$ Peter lernt Spanisch sprechen.

HPTS: Peter lernte, Spanisch sei eine romanische Sprache.

DASS: $\quad$ Peter hat <es> in der Schule gelernt, daß spanisch eine romanische Sprache ist.

FRAG: $\quad$ Peter lernte <es > in der Fahrschule, wie ein Motor funktioniert. 


\section{lesen}

Zesen

01

Zesen

$1: S E$ mit fakultativem

Korrelat

01

DASS :

HPTS :

Zesen

$0(1$

Zesen ( ̈̈ber - Akk)

\section{lieben}

Zieben

1:SE Zum Korrelat vgl. Einleitung 6.3.5.

\section{liefern}

Ziefern

Ziefern

$01(3$
FRAG :

P1 Der Priester liest die Messe.

P1 Hans hat die Nachricht gelesen.

Ich habe <es> in der Zeitung gelesen, daß sich ein Unfall ereignet hat.

Ich habe <es> in der Zeitung gelesen, wie sich der Unfall ereignet hat.

Ich habe <es> in der Zeitung gelesen, der Dieb soll entkommen sein.

P1 Evaliest (ein Buch).

P2 Der Professor Ziest (über Goethe).

P1 Romeo liebt Julia.

INF+: Er liebt es gar nicht, immer kritisiert zu werden.

DASS: $\quad$ Er liebt es gar nicht, daß seine Kinder bei den Großeltern verwöhnt werden.

P1 Hannibal liefert den Römern [in Norditalien] eine heftige Schlacht.

Die gegnerischen Truppen liefern sich eine Schlacht.

P1 Die Firma liefert (dem Bäcker) Mehl. 
Zieferm

\section{liegen}

Ziegen

O:SE Zum Korrelat vgl. Einleitung 6.3.5.

Ziegen an - Dat

$0: S E$ Zum Korrelat vgl. Einleitung 6.3.5.

O:SE Zum Korrelat vgl. Einleitung 6.3.5.

Ziegen bei - Dat

O:SE Zum Korrelat vgl. Einleitung 6.3.5.

Ziegen (mit-Dat)

Ziegen

Ziegen

Pertinenzelement im Dativ Ziegen
Der Unfall hat (mir) interessantes Material für meine Untersuchung geliefert.

PO Die Arbeit liegt ihm.

INF+: Es liegt ihr, Bilder zu malen.

Po Seine schlechte Laune lag am trüben Wetter.

INF+: $\quad$ Es liegt an dir, die Entscheidung zu treffen.

DASS: Es lag am Wetter, daß er schlechte Laune hatte.

DASS :

Meine schlechte Laune liegt daran, daß ihr mich geärgert habt.

Po Die Entscheidung liegt bei der Regierung.

INF+: Es liegt beim Arbeitsgericht, diesen Streitfall zu entscheiden.

PO Du liegst schief (mit deiner Meinung).

PO Köln liegt am Rhein.

Das Buch liegt auf dem Tisch.

Das Kind Ziegt [krank] im Bett.

PO Das Auto liegt gut auf der Straße.

Po Das Essen Ziegt ihm (schwer) im Magen.

Seine Gesundheit liegt mir (sehr) am Herzen.

PO Der Fall lag anders. 
*am Herzen Ziegen

*auf der Hand Ziegen

*im Blut liegen

*im Interesse Ziegen

*im Krieg liegen

*im Schlaf Ziegen

*im Sterben Ziegen

*im Streit liegen mit-Dat

\section{loben}

Zoben

1:SE mit fakultativem Korrelat

\section{lohnen}

Zohnen

1:SE Zum Korrelat vgl. Einleitung 6.3.5.

sich/Akk Zohnen

O:SE Zum Korrelat vgl. Einleitung 6.3.5.

lügen

Zügen
I

I

I

I

I

DASS :

$013 v 8$

INF+ :

$\mathrm{O}$
Po Deine Gesundheit liegt mir sehr am Herzen.

PO Dieser Verdacht liegt klar auf der Hand.

PO Der Rhythmus liegt ihm im Blut.

PO Das neue Gesetz liegt im Interesse der Mieter.

PO Die beiden Staaten liegen miteinander im Krieg.

PO Die Kinder Ziegen im Schlaf.

Po Der Patient liegt im Sterben.

Po Die Fomilie liegt im Streit mit den Nachbarn.

P1 Der Vater lobt seinen Sohn.

Der Vater lobt <es>, daß der Sohn in der Schule zu kritischem Denken erzogen wird.

P1 Er lohnt ihm seine Hilfe schlecht. Er hat mir meine Hilfe [nicht] gelohnt. Er hat deine Hilfe schlecht gelohnt. Du hast es mir schlecht gelohnt, daß ich dir geholfen habe.

Po Diese Arbeit Zohnt sich [nicht]. Es Zohnt sich, diesen Film anzusehen.

P2 Der Junge hat gelogen. 


\section{machen}

machen

machen

O:SE Zum Korrelat vgl. Einleitung 6.3 .5 .

machen zu - Dat

machen (aus - Dat)

machen

O:SE Zum Korrelat vgl. Einleitung 6.3.5.

1:SE Zum Korrelat vg1. Einleitung 6.3.5.

machen

O:SE Zum Korrelat vgl. Einleitung 6.3.5.

machen

06

sich/Akk machen
01

$01(3$

INF+:

DASS :

014

0114 .

018

INF+:

DASS :

INF+ :

$0(18$

INF+ :

DASS :
P1 Der Schuler macht seine Hausaufgabe.

Der Lehriing macht Möbel.

Die Hausfrau macht die Betten.

PO Das Spiez macht (mir) Spaß.

Es macht mir Spaß, Tischtennis zu spielen.

Es macht mir Mut, daß du mir helfen willst.

P1 Der Minister hat Herrn Schmidt zum Leiter dieser Abteilung gemacht.

P1 Der Bauer macht (aus Apfeln) Wein.

P1 Die Mutter macht die Suppe warm.

Es macht mich glücklich, dich wiederzusehen.

Es macht mir die Arbeit leichter, daß du mir dabei hilfst.

Du machst es mir schwer, dir zu helfen.

P1 Liebe macht (die Menschen) blind.

Es macht mich zufrieden, so viel Geld zu verdienen.

Es macht mich zufrieden, daß ich so viel Geld verdiene.

P2 Das Baby macht ins Bett.

po Euer Sohn hat sich gemacht. 
sich/Akk machen aus - Dat

4:SE mit obligator 1schem Korrelat

\section{BEMERKUNG :}

sich/Akk machen an - Akk

4:SE mit obligatorischem Korrelat

sich/Akk machen

malen

malen

Pertinenzelement im Dativ

malen

malen

meinen

meinen

1:SE ohne Korrelat

BEMERKUNG :

meinen (mit-Dat)
08

01116

018

INF+:

DASS :

04

INF+:

08

016

01

DASS :

HPTS :

$01(4$
PO Er macht sich nichts aus Kuchen.

Er macht sich nichts daraus, Kaffee zu trinken.

Er macht sich nichts daraus, daß er arbeiten muß.

$E$, meist indefinit oder $S E$.

PO Der Chef macht sich an die Arbeit.

Die Hausfrau macht sich daran, ihre Arbeiten zu erledigen.

Po Dein neues Kleid macht sich ausgezeichnet.

P1 Die Hippies malen ihren Freundinnen Blumen auf die Stirn.

Der Clown malt sich bunte Streifen ins Gesicht.

P1 Ermalt (bunte Blumen) (auf Japanpapier).

p1 Er malt den Baum abstrakt.

Po Ich meine etwas anderes.

Ich meine, daß wir nachhause fahren sollten.

Ich meine, wir sollten nachhause fahren.

$E$, meist indefinit oder $S E$.

PO (Mit diesen kritischen Worten) meint er seine Eltern. 
$1: \mathrm{SE}$ ohne Korrelat

DASS :

HPTS :

meinen es (mit-Dat)

$0(48$

4:SE mit obligatorischem Korrelat

meinen

INF+:

DASS :

09

Mit seinen kritischen Worten meinte er, daß seine Eltern diesmal nicht im Recht seien.

Mit diesen Worten meinte er, seine Eltern seien diesmal nicht im Recht.

PO Der Kollege hat es (mit seinem Rat) gut gemeint.

9: TNF+:

melden

mezden

01

1:SE mit fakultativem Korrelat

DASS :

HPTS :

Der junge Mann meint es ehrlich damit, das Mädchen heiraten zu wollen.

Der junge Mann meint es ehrlich domit, daß er das Mödchen heiraten wizl.

PO

Ich meinte fälschlicherweise, nachhause zu fahren.

Ich meinte fälschlicherweise, daß das Problem schon gelöst sei.

Ich meinte fälschlicherweise, das Problem sei schon gelöst.

P1 Der Wetterbericht meldet Regen.

DASS : Der Wetterbericht hat <es> schon gestern gemeldet, daß es heute regnen würde.

FRAG: Der Wetterbericht hat <es> nicht gemeldet, ob es heute Regen gibt.

HPTS: Der Wetterbericht hat <es > eben gemeldet, es gibt Regen. 
meZden

0113

1:SE mit fakultativem Korrelat

DASS :

FRAG :

HPTS :

melden

sich/Akk melden

016

sich/Akk melden

0

O (5

sich/Akk melden für-Akk

04

4:SE mit obligatorischem Korrelat

sich/Akk melden $z u$ - Dat

4:SE mit obligatorischem Korrelat

sich/Akk melden

06

krankmelden

01

\section{merken}

merken

INF+:

INF+:
P1 Ich melde (der Polizei) den Unfall.

Ich habe <es > der Polizei gemeldet, daß ich den Unfall beobachtet habe.

Ich werde <es > der Polizei melden, wo sich der Gesuchte versteckt.

In der Zeitung wurde <es> gestern schon gemeldet, der Minister wolle zurulcktreten.

P1 Der Botschafter meldet die Angelegenheit nach Bonn.

Po Der Hunger meldet sich.

Po. (Am Telefon) hat sich eine fremde Stimme gemeldet. Der Soldat meldet sich (bei seiner Kompanie).

Po Der Student meldet sich für einen Russischkurs. Der Student meldet sich dafür, eine Arbeit über Goethe zu schreiben.

PO Der Student meldet sich aum Russischkurs. Der Student meldet sich dazu, eine Arbeit über Goethe iu schreiben.

PO Der Soldat meldet sich an die Front.

P1 Der Schüler meldet sich krank.

PO Die Schüler merken nichts. 
1:SE mit fakultativem Korrelat

\section{BEMERKUNG :}

merken von-Dat

$4: \mathrm{SE}$ mit obligatorischem Korrelat

BEMERKUNG :

sich/Dat merken

1:SE mit fakultativem Korrelat

\section{messen}

messen

Pertinenzelement im Dativ $1: \mathrm{SE}$ ohne Korrelat
DASS :

FRAG :

HPTS :

\section{DASS :}

FRAG :

01

DASS :

FRAG :

\section{FRAG :}

Die Schüler merken 〈es >, daß der Lehrer sich ärgert.

Die Schüler merken 〈es>, ob der Lehrer sich ärgert.

Die Schüler merken $\langle e s\rangle$, der Lehrer ärgert sich. $\mathrm{E}_{1}$ meist indefinit oder $\mathrm{SE}$.

PO Von dem starken Sturm habe ich [nicht] viel gemerkt.

Man merkt nichts davon, daß du krank bist.

Man merkt nichts davon, wie schlecht es dir geht. $\mathrm{E}$, meist indefinit.

PO Die Großmutter hat sich diese Geschichte gemerkt. Ich wërde <es> mir merken, daß du mich angelogen hast.

Ich werde <es> mir nie merken, wie dein Freund heist.

P1 Hugo mißt die Lönge des Tisches.

Die Krankenschwester mißt das Fieber.

Sie mißt ihm den Puls.

Hugo hat gemessen, wie lang der Tisch ist. 
messen

messen

01

018

sich/Akk messen mit - Dat

04

mieten

mieten

01

\section{mißverstehen}

miBverstehen

\section{mitteilen}

mitteizen

$01(3$

1:SE mit fakultativem Korrelat

\section{PO Der Mann mißt [genau] $2 \mathrm{~m}$.}

P1 Der Vater mißt den Sohn mit bösen Blicken.

PO Der Sportler miBt sich mit seinem Rivalen.

P1 Das Ehepaar mietet eine Wohnung.

P1 Der Sohn mißversteht seinen Vater.

P1 Der Bundeskanzler teilt (den Ministern) die Entscheidung mit.

DASS: $\quad$ Er teizt <es> der Behörde mit, daß er seine Wohnung gewechselt hat.

FRAG : Er teilt <es> der Behörde mit, wann er umgezogen ist.

HPTS: $\quad$ Er teilt <es> der Behörde mit, er habe seine Wohnung gewechselt. 


\section{nachdenken}

nachdenken ( $\ddot{b} \mathrm{ber}-\mathrm{Akk}$ )

$0(4$

4:SE mit fakultativem Korrelat

4:SE mit obligatorischem Korrelat

nähen

nähen

Pertinenzelement im Dativ nähen

016

\section{nehmen}

nehmen

nehmen

$O: S E$

Zum Korrelat vgl. Einleitung 6.3.5.

nehmen $z u-D a t$

nehmen (von - Dat)

$01(4$

nehmen
014

01
P2 Ich denke (über den Vortrag) nach.

FRAG: $\quad$ Ich denke <darüber> nach, warum du das gesagt hast.

DASS: $\quad$ Ich denke darüber nach, daß sie nicht geantwortet haben.

P1 Der Schneider näht [gerade] (mein Abendkleid). Der Arzt hat mir die Wunde genäht.

P1 Er näht Knöpfe an das Hemd.

P1 Mein KolZege nimmt UrZaub. Mein Bruder nimmt [heute] das Motorrad.

P1 Der Baum nimmt der Blume das Licht.

INF+: Es nimmt mir die gute Laune, eine schlechte Nachricht zu hören.

DASS: $\quad$ Es kann mir meine gute Laune nicht nehmen, daß es heute regnet.

P1 Irina nimmt die linke Hand zum Schreiben.

P1 Dieser Arzt nimmt (von den ärmsten Patienten) kein Geld.

P1 Die Mutter nimmt das Kind bei der Hand. 
nehmen

Pertinenzelement im Dativ nehmen

nehmen

1:SE Zum Kórrelat vgl. Einleitung 6.3.5.

*beim Wort nehmen mit-Dat T

*in Angriff nehmen T

*in Anspruch nehmen T

*in Augenschein nehmen ' T

*in Beschlag, nehmen T

*in Besitz nehmen T

*in Betrieb nehmen $T$

*in Empfang nehmen T

*ins Gebet nehmen T

*in Gebrauch nehmen T

*in Gewahrsam nehmen T

*in Haft nehmen T

*in die Hand nehmen T
P1 Die Mutter nimmt das Kind in die Arme.

Die Mutter nimmt dem Kind die Schere aus der Hand.

P1 Hans nimmt das Buch als Unterlage.

P1 Er nimmt seine Krankheit schwer.

INF+: $\quad$ Er nimmt es schwer, seinen Arbeitsplatz zu verizeren.

DASS: $\quad$ Er nimmt es schwer, daß er nicht mitfahren kann.

P1 Ich nehme dich mit deinem Versprechen beim Wort.

P1 Der Lehrer nimmt die schwierige Arbeit in Angriff.

P1 Der Kunde nimmt sein Recht in Anspruch.

P1 Der Höndler nimmt die Ware in Augenschein.

P1 Die Kinder nehmen das ganze Zimmer in Beschlag.

P1 Die Bauern nahmen das Land in Besitz.

P1 Der Meister hat die neue Maschine in Betrieb genommen.

P1 Die Gewinner des Preisrätsels nehmen die Preise in Empfang.

P1 Der Lehrer nimmt den verantwortungslosen Schüler ins Gebet.

P1 Die Firma nimmt die neue Rechenanlage in Gebrauch.

P1 Die Polizei nimmt den Dieb in Gewahrsam.

P1 Die Polizisten nahmen mehrere Demonstranten in Haft.

P1 Ich werde die Sache selbst in die Hand nehmen. 
* in Kauf nehmen

*in Obhut nehmen

$\mathrm{T}$

*in Schutz nehmen

*ins Verhör nehmen

*in Verwahruing nehmen

*in Zahlung nehmen

*zum Anlaß nehmen für - Akk

*zur Frau nehmen

*zur Kenntnis nehmen

*zum Mann nehmen

*zum Partner nehmen

*zum Vorwand nehmen für-Akk

*auf sich/Akk nehmen

*sich/Akk in acht nehmen

mit - Dat

*sich/ Akk in acht nehmen

vor - Dat

*zu sich/Dat nehmen

*<sich/Dat> zum Vorbild nehmen
P1 Man nimmt bei diesem Projekt Fehler in Kauf.

P1 Die alte Dame nimmt das Kind der Nachbarin in Obhut.

P1 Er nimmt seinen Freund gegen meine Vorwurfe in Schutz.

P1 Der Kommissar nimmt den Verdächtigen ins Verhör.

P1 Die Großmutter nimmt das Geld in Verwahrung.

P1 Der Autohändler nimmt den alten. Wagen in Zahlung.

P1 Der Minister nimmt die Umweltverschmutzung zum Anlaß für eine Gesetzesvorlage.

P1 Robert nimmt Ursula zur Frau.

P1 Hiermit nehme ich ihre Beschwerde zur Kenntnis.

P1 Ursula nimmt Fridolin zum Mann.

P1 Der Rechtsarwalt nimmt seinen Freund zum Partner.

P1 Er nimmt die Krankheit zum Vorwand für seine Trägheit.

PO Er nimmt die Schuld ouf sich.

PO Nimm dich in acht mit dem, was du sagst!

PO Er nimmt sich vor seinen Feinden in acht.

PO Das Mädchen nimmt eine Mahlzeit zu sich.

P1 Der kleine Junge nimmt <sich> seinen großen Bruder zum Vorbild. 


\section{nennen}

nennen

0113

nennen nach-Dat

014

nennen

1:SE Zum Korrelat vgl. Einleitung 6.3 .5 .

nennen

1:SE Zum Korrelat vgl. Einleitung 6.3.5.

\section{nützen}

nützen

01

nützen

$03 v 8$

017

018
INF+ :

DASS :

INF+:

DASS :

P1 Ich nütse die Gelegenheit.

PO Sein Fleiß hat ihm viel genütat.

Sein Fleiß hat ihm genützt.

Sein Fleiß hat nichts genützt.

$\mathrm{E}_{8}$ meist indefinit. 


\section{öffnen}

offnen

01

Pertinenzelement im Dativ offnen

sich/Akk öffnen

0

\section{operieren}

operieren

operieren

Pertinenzelement im Dativ operieren mit-Dat

operieren

\section{ordnen}

ordnen

ordnen
$01(8$
P1 Der Hausmeister öffnet die Tür. Er öffnet ihr das Kleid.

P2 Das Geschäft öffnet um 9 Uhr.

PO Ihr Mund öffnet sich.

P1 Der Arzt operiert (den Kranken).

P1 Er operiert ihm den Splitter aus dem Kopf. Der Chirurg operiert ihm die Kugel aus dem Arm.

P2 Der Trainer operiert mit einer überraschenden Taktik.

Po Die Schiffe operieren in der Nordsee.

P1 Er ordnet seine Angelegenheiten.

P1 Wir ordnen die Bücher (nach Verfassern). 


\section{packen}

packen

01

packen

$0(1$

packen

016

\section{parken}

parken

parken

$01(5$

05

passen

passen

o

passen

016

passen

passen zu-Dat

O:SE Zum Korrelat vgl. Einleitung 6.3 .5 .

passen auf - Akk

passen
P1 Die Katze packt den Hund.

Das Konzert packt mich.

Das Fieber packt den Kranken.

P1 Die Ehefrau packt (ihre Koffer).

P1 Der Student packt die Bücher in die Kiste.

P1 Ich parke mein Auto (vor der Garage).

Po Das Auto parkt vor der Garage.

P2 Ich passe.

P1 Er paßt den Schlüssel in das Schloß.

PO Der Anzug paßt (ihm).

PO Dieses Verhalten paßt zu diesem Mann. INF+: Es paßt zu ihm, den Clown zu spielen.

DASS: $\quad$ Es paßt zu ihm, daß er jegliche Hilfe ablehnt.

Po Die Beschreibung paßt auf den Mann.

PO Der Schrank paßt in dieses Zimmer. 


\section{passieren}

passieren

01

passieren

$01(6$

BEMERKUNG :

\section{passieren}

passieren

O:SE Zum Korrelat vgl. Einleitung 6.3.5.

passieren

BEMERKUNG :

\section{pflanzen}

pflanzen

\section{pflegen}

pflegen

01

\section{platzen}

platzen

platzen
P1 Der Reisende passiert die Grenze.

P1 Die Frau passiert Apfel (durch ein Tuch). STAMMFORMEN: passieren, passierte, hat passiert.

Po Das Unglück passierte (mir).

DASS: $\quad$ Es passiert mir oft, daß ich meine Brille vergesse.

PO (An dieser gefährlichen Kreuzung) sind schon viele Unfälle passiert.

STAMMFORMEN: passieren, passierte, ist passiert.

P1 Die Arbeiter pflanzen Bäume (an die Straße).

P1 Anna pflegt ihre kranke Schwester. sie pflegt sich.

Po Der Luftbalzon platzt.

PO Er platzt in die Gesellschaft hinein. 


\section{probieren}

probieren

1:SE ohne Korrelat

01

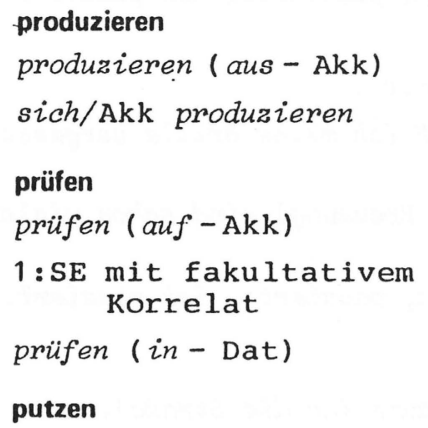

Pertinenzelement im Dativ putzen

putzen

sich/Akk putzen
P1 Hans probiert den Kaffee.

FRAG: Hans probiert, ob der Kaffee schmeckt.

P1 Die Fabrik produziert (aus Kunststoff) Lampenschirme.

Po Hans produziert sich (vor seinen Freunden).

P1 Der Kaufmann prüft die Rechnung (auf Fehler). Der Gast prüft 〈es〉, ob die Rechnung stimnt.

P1 Der Lehrer prüft (die Kinder) (in zwei Fächern).

P1 Klara putzt ihre Schuhe. Hans putzt sich die Nase.

P1 Die Hausfrau putzt (das Fenster).

P1 Die Hausfrau putzt den Schnee vom Fenster.

Po Die Dame putat sich. 


\section{radfahren}

radfahren

O

01

rasieren

Pertinenzelement im Dativ

\section{raten}

raten

$1: S E$ ohne Korrelat

raten $z u$ - Dat

4:SE mit fakultativem Korrelat

01

' FRAG :

$O(34$

INF+

HPTS :

raten

038

\section{rauchen}

rauchen

0

Pertinenzelement im Dativ rauchen
P2 Das Mädchen fährt rad.

P1 Der Friseur rasiert Emiz. Emil rasiert sich.

Er rasiert ihm den Bart.

P1 Die Kinder raten die Aufgabe. Die Kinder raten, ob die Sache richtig ist.

P2 Der Bauer rät (der Hausfrau) zum Kauf von Kartoffe $Z_{n}$. Der Bauer rät der Hausfrau 〈dazu>, Kartoffeln zu kaufen.

Der Bauer rät der Hausfrau 〈dazu>, sie solle Kartoffeln kaufen.

po Ich rate dir gut.

Po Der Ofen raucht. Mir raucht der Kopf.

P1 Hans raucht (Zigaretten). 


\section{rechnen}

rechnen

O (1

rechnen zu-Dat

014

1:SE Zum Korrelat vgl. Einleitung 6.3.5.

rechnen mit - Dat

4:SE mit obligatorischem Korrelat

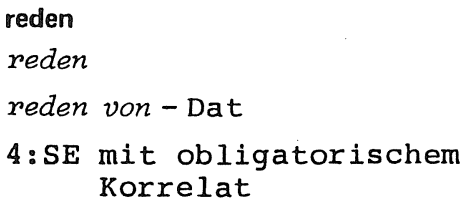

reden mit-Dat uber-Akk

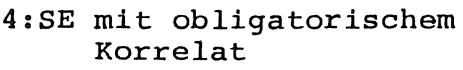

P1 Der Schüler rechnet (vier Aufgaben).

P1 Ich rechne ihn zu meinen Freunden.

INF+: Ich rechne es zu meinen größten Erfolgen, diese Stelle bekommen zu haben.

DASS :

Ich rechne es zu deinen größten Fehlern, daß du immer zu spät kommst.

P2 Ich rechne mit verschiedenen Möglichkeiten.

INF+ : Ich rechne damit, Geld zu bekommen.

DASS : Ich rechne damit, daß ich Geld bekomme.

P1 Er redet (Unsinn).

P2 Herr Mülzer redet vom Unfall seiner Tochter.

DASS: $\quad$ Er redet davon, daß seine Tochter einen Unfazl gehabt hat.

FRAG: Wir reden davon, wann wir in Urlaub fahren.

$04 v 4$

P2 Die Mutter redet mit ihrem Kind über das Buch. Die Mutter redet mit ihrem Kind.

Die Mutter redet über das Buch.

DASS : $\quad$ Ich werde mit meinen Eltern darüber reden, daß ich mehr Geld brauche.

FRAG: $\quad$ Ich werde mit meinen Eltern darüber reden, ob ich allein in Urlaub fahren darf. 
$04(8$

reden von - Dat

$04(8$

regieren

regieren

01

regieren

\section{regnen}

regnen (es)

regnen (es)

regnen (es)

Pertinenzelement im Dativ

\section{reinigen}

reinigen

Pertinenzelement im Dativ

reinigen

reinigen (von-Dat)

reisen

reisen (in-Dat)

$O(4$

$0(6$

reisen reden über - Akk

P2 Die Kinder reden (schlecht) über die Schule.

P2 Er redet (schlecht) von seinen Kollegen.

P1 Diese Partei regierte das Land [20 Jahre Iang].

P2 In diesem Land regiert [schon seit 20 Jahren] die gleiche Partei.

PO Es regnet.

PO Es regnet Prozesse.

PO Es hat ins Zimmer geregnet.

Es regnet ihm ouf den Kopf.

P1 [Morgen] werde ich das Zimmer reinigen. Ich reinige mir die Hände.

O(1 P1 Seife reinigt (die Hände).

01 (4) P1 Die Hausfrau reinigt die Fenster (vom Schnee).
PO Der Vertreter reist (in Stoffen).

P2 Er reist (nach Italien). 


\section{reiten}

reiten

BEMERKUNG :

\section{reiten}

reiten

BEMERKUNG :

reparieren

reservieren

reservieren

\section{retten}

retten

Pertinenzelement im Dativ retten (aus - Dat)

retten (vor - Dat)

$01(4$

retten

$01(6$

01

\section{riechen}

riechen

\section{reparieren}

P1 Er reitet (einen Rappen).

STAMMFORMEN: reiten, ritt, hat geritten.

P2 Hans reitet (in den Wald).

STAMMFORMEN: reiten, ritt, ist geritten.

P1 Ich habe meine Uhr repariert.

P1 Der Kellner reserviert den Tisch ( $a b$ neun).

P1 Deine Vorsicht hat mir das Leben gerettet. Die Operation hat mir die Hand gerettet.

P1 Hans rettet Franz (aus der Not).

P1 Der Arzt rettet den Kranken (vor dem Tod).

P1 Die Bauern retten ihr Vieh (in die Berge). Die Eirwohner retten sich (auf die Dächer). 


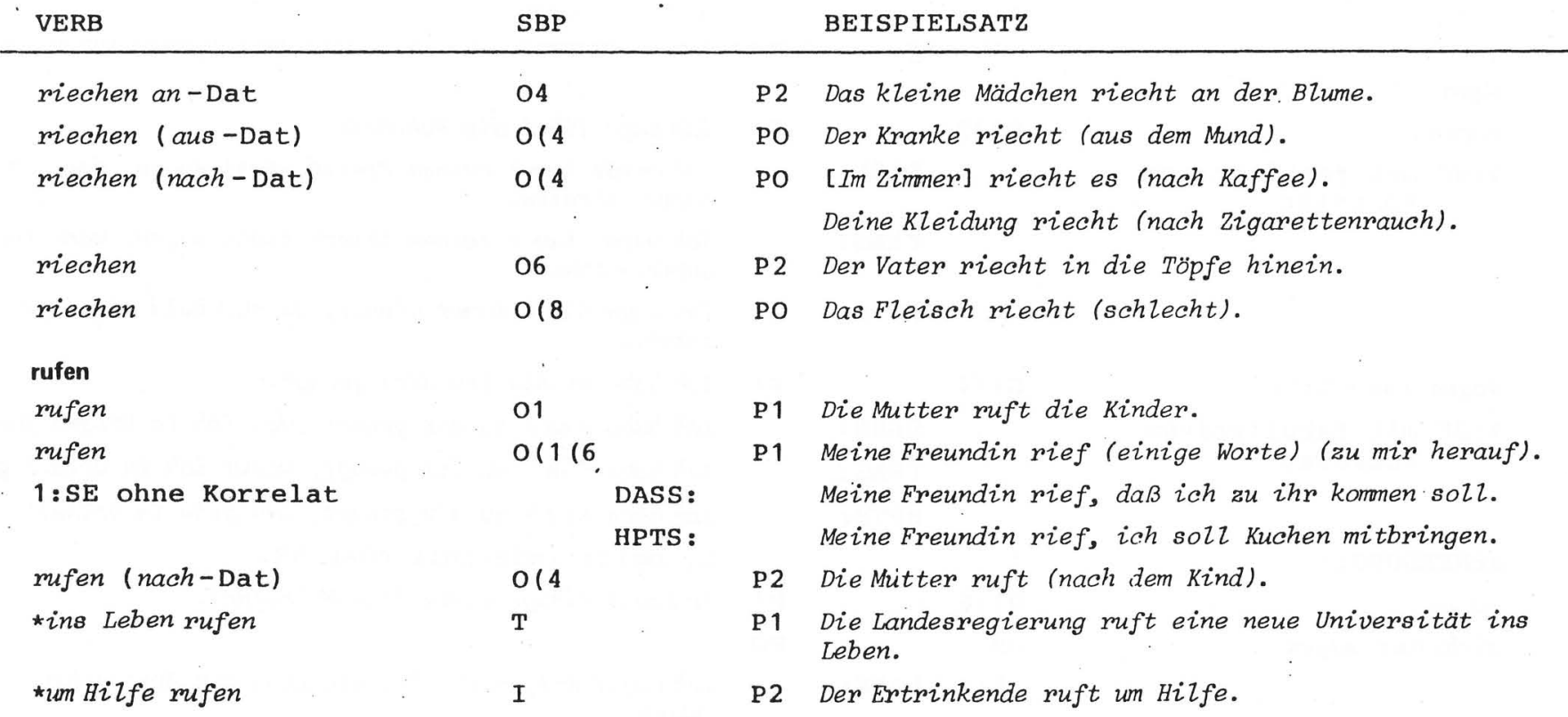




\section{sagen}

sagen

$1:$ SE mit fakultativem Korrelat

$01(3$

\section{DASS :}

FRAG :

HPTS :

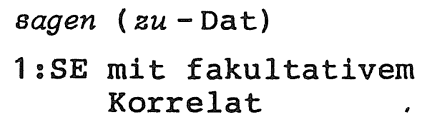

BEMERKUNG :

sagen

sich/Dat sagen

$01(6$

09

9 DASS :

HPTS :

\section{sammeln}

sarme $\mathrm{In}$

01

sarmeln
P1 Ich sage (ihm) die Wahrheit. Ich werde <es > seinem Freund nicht sagen, daß ich morgen abreise.

Ich werde <es > seinen Eltern nicht sagen, wann ich zurückkomme.

Ich sage $\langle$ es > immer wieder, es muß bald etwas geschehen.

P1 Ich habe nichts (zu dir) gesagt.

DASS: $\quad$ Ich habe <es> zu dir gesagt, daß ich in Urlaub fahre.

FRAG: Ich habe <es> zu dir gesagt, warum ich in Urlaub gehe.

HPTS: Ich habe <es> zu dir gesagt, ich gehe in Uriaub. $\mathrm{E}_{1}$ meist indefinit oder SE.

P1 Er sagte einige Worte (ins Mikrofon).

PO

Ich sagte mir, daß ich heute noch zum Arzt gehen mulsse.

Er sagte sich, er müsse die Angelegenheit heute noch erledigen.

P1 Er sammelt Briefmarken.

PO Er sammelt seine Gedanken. 
sammeln ( für - Akk)

sich/Akk sammeln

sich/Akk sammeln

$0(1) 4$

0

015

schaden

schaden

\section{schalten}

schalten

schalten auf - Akk

schalten (in-Akk)

schalten

\section{scheinen}

scheinen

Pertinenzelement im Dativ scheinen

schenken

schenken

schenken

sich/Dat schenken

$1: \mathrm{SE}$ Zum Korrelat vgl. Einleitung 6.3.5.

013

$01(3$

01
P1 Die Kinder sammeln (Geld) (für das Rote Kreuz).

PO Der Redner sammelt sich.

PO Das Wasser sammelt sich (an der tiefsten Stelle).

P2 Die Sonne schadet seiner Gesundheit.

P1 Die Post schaltet neue Telefonleitungen.

P1 Die Hausfrau schaltet den ofen auf drei.

P2 Der Fahrer, chaltet (in den dritten Gang).

P2 Die Schüler haben schnell geschaltet.

Po Die Sonne scheint (in unser Zimmer herein). Die Sonne scheint.ihm ins Gesicht.

PO Die Sache scheint klar.

P1 Ich schenke diesem Kunstwerk keine Aufmerksamkeit.

P1 Ich schenke (ihm) Blumen.

PO [Heute] schenke ich mir die Hausaufgaben.

INF+: Heute schenke ich es mir, Hausaufgaben zu machen. 


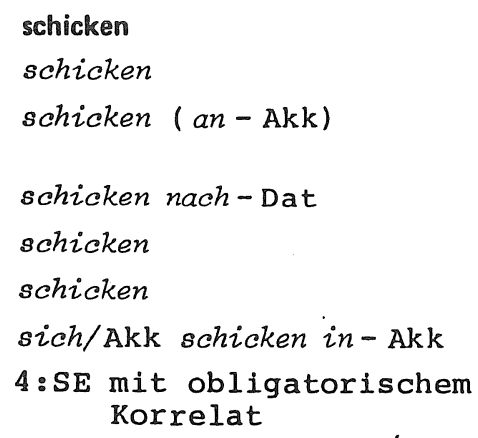

P1 Karl hat (mir) die Fotos geschickt.

P1 Meine Schwester hat (an ihre Freunde) eine Einladung geschickt.

P1 Die Eltern schickten (den Nachbarn) nach dem Arzt.

P1 Die Mutter schickt die Kinder ins Bett.

P1 Karl hat die Fotos (nach Mannheim) geschickt.

Po Der junge Mann schickt sich in seine Krankheit.

INF+: Er hat sich darein geschickt, nur noch wenige Monate leben zu dürfen.

DASS: $\quad$ Der Kranke hat sich darein geschickt, daß er nur noch wenige Monate zu leben hat.

PO Solches Benehmen schickt sich [nicht] (für Pfarrer).

P1 Der kleine Junge schiebt die Schuld auf seinen Freund.

P1 Hans schiebt sein Auto (in die Garage).

po Hans schiebt die Schuld von sich.

P1 Seine Freunde schimpfen ihn einen dummen Jungen.

P2 Die Mutter schimpft (auf die Kinder). 
schimpfen ( Über - Akk)

4:SE mit fakultativem Korrelat

014

DASS :

HPTS :

\section{schlafen}

schlafen

0

schlafen

01

schlafen mit-Dat

04

schlagen

schlagen

0

schlagen

01

schlagen

schlagen über - Akk

011

014

schlagen

016

Pertinenzelement im Dativ schlagen
P2 Die Arbeiter schimpfen (über den niedrigen Lohn). Die Bauern schimpfen 〈darüber >, daß die Kartoffeln zubillig seien.

Die Bauern schimpfen 〈darüber>, die Kartoffeln seien zu bizzig.

P2 Das Kind schläft.

PO Er schläft einen tiefen Schlaf.

Po Er schläft mit seiner Freundin.

Po Die Nachtigalz schlägt.

Seine Stunde hat geschlagen.

P1 Albert schlägt seinen Freund.

Der Löwe schlägt die Antilope.

PO Die Uhr schlägt (zwei).

P1 Er schlägt die Beine übereinander.

P1 Lieschen schlägt awei Eier in die Schüssel. Der Schüler schlägt den Ball aus dem Feld.

Hans schlägt Lieschen den Ball aus der Hand.

01 (61 Hans schlägt ein Loch (in die Wand). 


schlagen
schlagen
schlagen für-Akk
schlagen mit-Dat
schlagen
schlagen
Pertinenzelement im
Akkusativ
Pertinenzelement im Dati
sich/Akk schlagen für-Akk
sich/Akk schlagen mit-Dat
um-Akk
4 SE mit obligatorischem
Korrelat

sich/Akk schlagen

*in die Flucht schlagen
04

$04 v 4$

\section{INF+ :}

FRAG :

08
P1 Hans schlägt das teure Porzellan kaputt.

P1 Krefeld schlägt Düsseldorf (5:2).

PO Sein Herz schlägt [nur] für Amalie.

P2 Der Vogel schlägt mit den Flügeln.

Po Das Feuer schlägt aus dem ofen. Die Zweige schlagen gegen das Fenster.

P2 Gerhard schlägt nach dem Hund. Er schlägt ihn ins Gesicht.

Er schlägt ihm ins Gesicht.

PO Franz schlägt sich für seine Freunde.

PO Bernd schlägt sich mit Günther um sein Recht. Gerd schlägt sich mit seinem Bruder. Die Kinder schlagen sich um das Spielzeug. Die Kinder schlagen sich darum, ins Kino gehen zu dürfen.

Die Jungen schlagen sich darum, wer mit dem Fahrrad fahren darf.

PO Er schlägt sich gut.

P1 Der Hund schlägt die Katze in die Flucht. 
*sich/Akk ouf die Seite

schlagen

I

BEMERKUNG :

schlagen

schlagen nach - Dat

04

BEMERKUNG :

\section{schließen}

schließen

Pertinenzelement im Dativ schließen aus - Dat

1:SE ohne Korrelat

\section{1:SE mit fakultativem Korrelat}

\section{BEMERKUNG :}

schließen

schließen mit-Dat

4:SE mit obligatorischem Korrelat

schließen von-Dat ouf-Akk

016

04 po Er schlägt sich auf die Seite seines Freundes.

STAMMFORMEN: schlagen, schlug, hat geschlagen.

PO Der Sohn schlägt [ganz] nach dem Vater.

STAMMFORMEN: schlagen, schlug, ist geschlagen.

P1 Er schließt das Geschäft.

Der Vorsitzende schließt die Versammlung.

Er schließt dem Toten die Augen.

P1 Man kann aus seinem Brief [nicht] viel schließen.

FRAG: Sie schließt aus seinem Brief, warum er nicht kommt.

HPTS: $\quad$ Sie schließt aus seinem Brief, er liebe sie.

DASS: $\quad$ Sie schließt <es> aus seinen Briefen, daß er sie liebt.

$\mathrm{E}_{1}$ meist indefinit oder $\mathrm{SE}$.

P1 Die Mutter schließt das Brot in den Schrank.

PO Das Essen schloß mit einem Kaffee.

DASS: Das Essen schloß damit, daß man Kaffee trank.

P2 Du schließt von dir auf andere. 


\section{schließen}

05

schließen

*einen Vertrag schließen

mit - Dat

018

I

\section{schmecken}

schmecken

01

schmecken

schmecken (nach-Dat)

schneiden

schneiden

Pertinenzelement im Dativ schneiden

Pertinenzelement im

Akkusativ

Pertinenzelement im Dativ schneiden

\section{schneien}

schneien (es)

schneien (es)

Pertinenzelement im Dativ
P2 Das Milchgeschäft schließt um ein Uhr.

Po Die Tür schließt (gut).

PO Israel hat mit Ägypten einen erneuten Waffenstillstandsvertrag geschlossen.

PO Ich schmecke das Salz.

PO Die Suppe schmeckt (mir) (gut).

Po Die Suppe schmeckt (nach Salz).

P1 Die Mutter schneidet das Brot. Die Mutter schneidet sich. Der Frisör schneidet ihm die Haare.

P 2 Ich habe mir in den Finger geschnitten. Ich habe mich in den Finger geschnitten.

Ich habe mir in den Finger geschnitten.

Po Das Messer schneidet (gut).

PO Es schneit.

PO Es schneit ins Zimmer.

Es schneit mir ins Gesicht. 


\section{BEMERKUNG：}

\section{schneien}

schneien

06

BEMERKUNG :

\section{schreiben}

schreiben

01

schreiben

011

schreiben

$01 v 3$

\section{$1:$ SE mit fakultativem Korrelat}

schreiben an-Akk

schreiben

$01 \mathrm{v} 4$
STAMMFORMEN : schneien, schneite, hat geschneit.

PO Sie schneiten [uns] [plötzlich] ins Haus. STAMMFORMEN : schneien, schneite, ist geschneit.

P2 Die Sekretärin schreibt Schreibmaschine.

P1 Der junge Mann schreibt (ein Buch).

P1 Er schreibt seiner Mutter einen Brief. Er schreibt einen Brief. Er schreibt seiner Mutter.

DASS : FRAG : HPTS : Er schreibt <es> seiner Mutter, daß er heiraten will. Er schreibt <es > seiner Mutter, wann er kommt. Er hat <es> ihr geschrieben, er werde bald kommen.

P1 Er schreibt einen Brief an seine Mutter. Er schreibt einen Brief. Er schreibt an seine Mutter.

P1 Der Junge schreibt die Arbeit ins Heft. Er schreibt eine Arbeit.

Der Junge schreibt auf ein Blatt Papier. 
1:SE mit fakultativem Korrelat

\section{1:SE ohne Korrelat}

schreiben

schreiben (an - Dat)

\section{schützen}

schützen gegen - Akk

4:SE mit obligatorischem Korrelat

schützen vor - Dat

4:SE mit obligatorischem Korrelat 01v4

DASS :

FRAG:

HPTS :

018

$O(4$

INF+ :

DASS :

$01 v 4$

INF+ :

DASS :
Ich habe <es > neben den Satz geschrieben, daß er nicht stimmt.

Ich habe <es> auf den Zettel geschrieben, wohin ich gehe.

Ich habe daneben geschrieben, dieser Satz stimmt nicht.

P1 Er schrieb das Wort falsch.

Man schreibt ihren Nomen mit $h$.

P2 Hans schreibt (an einem Buch).

P1 Diese Medizin schützt das Kind gegen Schnupfen. Die Polizei schützt das Leben der Einwohner. Diese Medizin schütat gegen Schnupfen.

Die Medizin schütat uns dagegen, Schnupfen zu bekommen.

Die Medizin schützt das Kind dagegen, daß es krank wird.

P1 Der Regenschirm schützt das Mädchen vor dem Regen. Der Regenschirm schützt die Haare.

Der Regenschirm schützt vor dem Regen.

Der Regenschirm schütat ihn davor, naß zu werden. Der Regenschirm schützt das Mädchen davor, daß es naß wird. 


\section{schwimmen}

schwimmen.

BEMERKUNG :

\section{schwimmen}

schwimmen

schwinmen

schwimmen

BEMERKUNG :

\section{schwitzen}

schwitzen

\section{sehen}

sehen

1:SE ohne Korrelat

1:SE mit fakultativem Korrelat

sehen in - Dat

o

01
Po Das Kalbsschnitzel schwinmt im Fett.

STAMMFORMEN : schwimmen, schwarm, hat geschwommen.

PO Holz schwimmt.

01 P1 Der Sportler schwimmt einen Weltrekord.

016

PO Er schwimnt (über den See).

STAMMFORMEN : schwimmen, schwamm, ist geschwommen.

PO Er schwitzt [am ganzen Körper].

P1 Ich sehe ihn. Ich sehe [noch] eine Moglichkeit.

INF-: $\quad$ Ich sehe den Mann kommen.

DASS: $\quad$ Ich sehe $\langle e s\rangle$, daß er kormt.

FRAG: Ich sehe $\langle e s\rangle$, woher er kommt.

HPTS: Ich sehe $\langle e s\rangle$, er kommt.

014 P1 Ich sehe in ihm einen Freund. 
4:SE mit obligatorischem Korrelat

sehen

018

sehen nach - Dat

04

sehen

06

Pertinenzelement im Dativ sehen

sein

sein

sein an - Dat

$\mathrm{O} 2$

04

O:SE Zum Korrelat vgl. Einleitung 6.3.5.

sein aus - Dat

sein für - Akk

4:SE mit obligatorischem Korrelat

sein gegen - Akk
INF+:

DASS :

FRAG: $\quad$ Ich sehe ein Problem darin, wie wir diese Frage behandeln sollen.

Ich sehe einen Vorteil darin, nahe bei der Universität zu wohnen.

Ich sehe einen Vorteil darin, daß ich nahe bei der Universität wohne.

P1 Du siehst die Dinge [nicht] richtig.

PO Die Mutter sieht nach den Kindern.

Po Sie sieht auf die Uhr.

Sie sieht ihm [tief] in die Augen.

PO Der alte Mann sieht sehr schlecht.

PO Ich bin deiner Meinung.

PO Die Reihe ist an dir.

INF+: Es ist an dir, über dieses Problem zu entscheiden.

PO Der Schreibtisch ist aus Holz.

Po Der Minister war für eine allgemeine Geschwindigkeitsbeschränkung.

INF+: $\quad$ Wir sind dafür, am Donnerstag ins Kino zu gehen.

DASS: $\quad$ Die Eltern sind dafür, daß ihre Tochter auf das Gymnasium kommt.

PO Diemeisten Arzte sind gegen das Rauchen. 


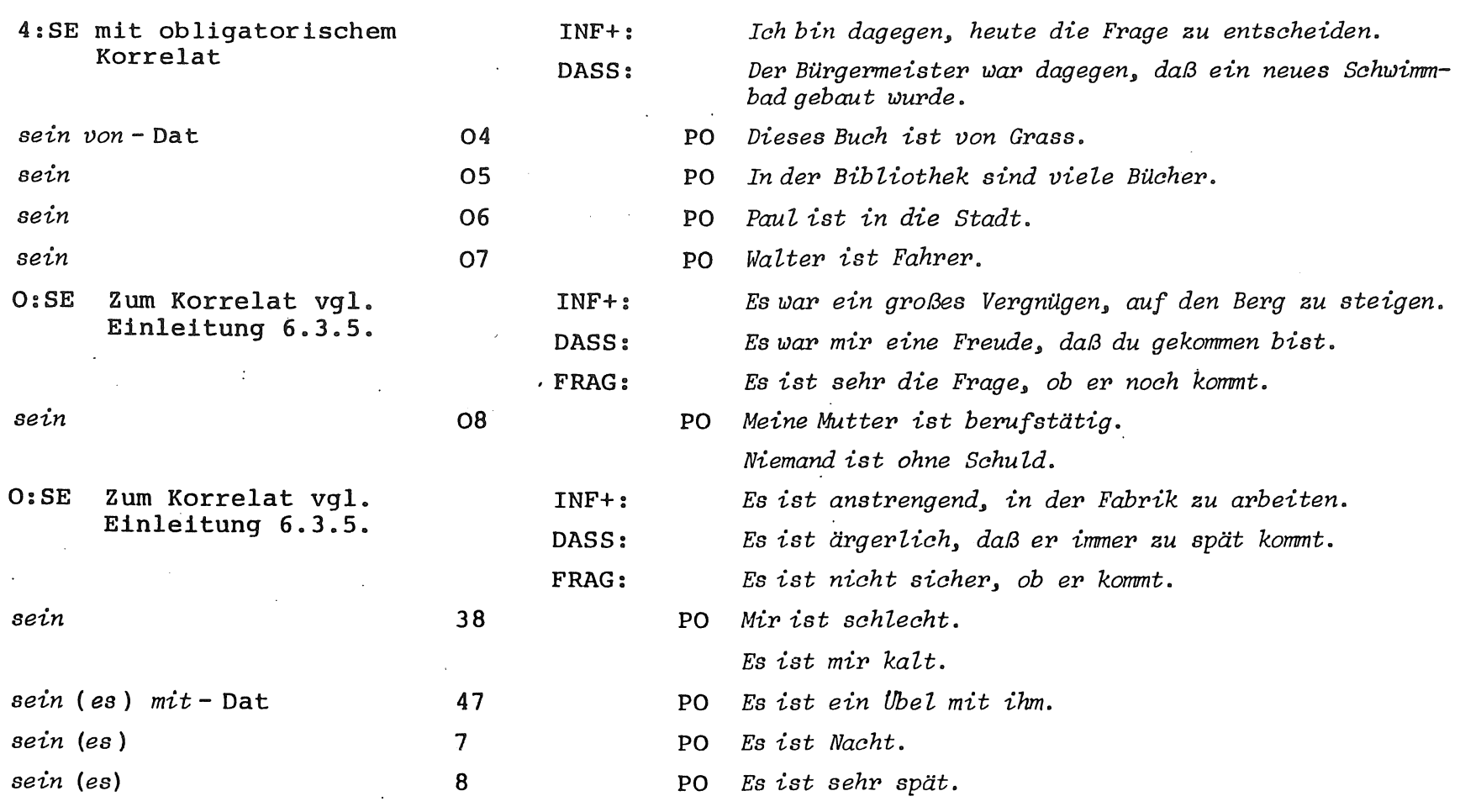


*auf dem Zaufenden sein in-Dat I

*auf dem Zaufenden sein mit-Dat I *auf der Hut sein vor- Dat I *außer Betrieb sein I *außer Kraft sein I *außer Ubung sein I *im Anzug sein I

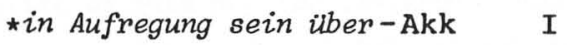

*in Aufregung sein wegen'-Gen I

$\star$ im Bau sein

\section{I}

*in Bedrängnis sein

$\star$ im Begriff sein

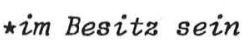

*in Betrieb sein

$\star$ in Bewegung sein

*im Druck sein

*in Eile sein
Po Paul ist in politischen Fragen immer ouf dem Iaufenden.

PO Ich bin endlich mit meiner Arbeit auf dem Zaufenden.

Po Der Einbrecher war vor dem Wachhund auf der Hut.

Po Der Aufzug ist außer Betrieb.

PO Dieses Gesetz ist schon seit Jahren außer Kraft.

PO Der Sportler ist außer Ubung.

PO Ein Gewitter ist im Anzug.

PO Die Eltern sind in heller Aufregung über das Ausbleiben ihres sohnes.

po Die ganze Familie ist in Aufregung wegen Annas Krankheit.

Po Das Schulgebäude ist noch im Bau.

PO Der Prüfling ist in großer Bedrängnis.

Po Die Gäste sind im Begriff, nach Hause zu fahren.

Po Der Politiker ist im Besitz wichtiger Informationen. Wichtige Informationen sind im Besitz des Politikers.

Po Die neue Datenverarbeitungsanlage ist schon in Betrieb.

PO Die Menschenmenge ist in Bewegung.

po Ich bin mit meiner Arbeit sehr im Druck. Ein neues Werk dieses Wissenschaftlers ist im Druck.

PO Der Geschäfsmann ist in großer Eile. 
$\star$ in FZuß sein

$\star$ im Gang(e) sein

*in Gefahr sein

*im Gespräch sein

*im Glauben sein

$\star$ im Gleichgewicht sein

*in Kraft sein

*in der Lage sein zu-Dat

*in Not sein

*in Ordnung sein

*in Ubung sein

*in Unordnung sein
PO Die innerdeutschen Verhandlungen sind noch in Fluß.

Po Die Vorbereitungen für das Fest sind noch in vollem Gang (e).

PO Sein Leben ist in Gefahr. Der Spekulant ist in Gefahr, sein ganzes Vermögen zu verlieren.

Po Die Einführung einer neuen Kraftfahrzeugsteuer ist im Gespräch.

Po Der Student ist im festen Glauben, das Examen nicht bestanden zu haben.

PO Das Kräfteverhältnis der Großmächte ist im Gleichgewicht.

Po Die neue Straßenverkehrsordnung ist seit März 1971 in Kraft.

PO Du bist zu besseren Leistungen in der Lage.

Po Die Opfer der Uberschwemmung waren in großer Not.

Po Das Zimmer ist in Ordnung.

Egon ist schwer in Ordnung.

Mit seinem Auto ist etwas nicht in Ordnung.

Po Die Demonstranten sind im Recht mit ihren Forderungen.

PO Der Sportler ist nicht mehr in Ubung.

Po Deine Kleidung ist in Unordnung. 
*im Unrecht sein mit-Dat

*in Versuchung sein

*im Verzug sein

I

*im Verzug sein mit-Dat

I

*im Zweifel sein über-Akk

I

*zu Ende sein

I

*außer sich/Dat sein (über - Akk)

*sich/Dat im klaren sein über - Akk

*sich/Dat im unklaren sein über - Akk

\section{senden}

senden

senden an-Akk

014

senden

016

BEMERKUNG :

\section{senden}

senden

BEMERKUNG :
PO Der Polizist war im Unrecht mit seiner Behauptung.

Po Der Kunde ist in Versuchung, im Warenhaus etwas zu stehlen.

PO Gefahr ist im Verzug.

PO Ich bin mit meiner Arbeit im Verzug.

PO Der Prüfer ist im Zweifel über die wissenschaftlichen Fähigkeiten seines Studenten.

PO Die Ausstellung ist im Oktober zu Ende.

PO Der Vater ist außer sich über die schlechten Zensuren seines Sohnes.

PO Wir sind uns über unsere Aufgaben im klaren.

Po Uber meine Zukunft bin ich mir im unklaren.

P1 Die Mutter sendet ihrem Sohn ein Paket.

P1 Die Mutter sendet ein Paket an ihren Sohn.

P1 Die Mutter sendet ein Paket nach Frankfurt. STAMMFORMEN: senden, sandte, hat gesandt.

P1 Der Saarzündische. Rundfunk sendet ein Konzert. STAMMFORMEN: senden, sendete, hat gesendet. 


\section{setzen}

setzen

setzen auf-Akk

setzen auf-Akk

4:SE mit obligatorischem Korrelat

setzen

setzen

setzen (es)

sich/Akk setzen

sich/Akk setzen

sich/Akk setzen

Pertinenzelement im Dativ

instandsetzen

*außer Kraft setzen

*in Betrieb setzen

*in Bewegung setzen

*in Beziehung setzen zu-Dat

*in Brand setzen
01

014

$O(14$

DASS :

016

06

1

0

06

o 16

\section{1}

$\mathrm{T}$

T

$\mathrm{T}$

$\mathrm{T}$

$\mathbf{T}$
P1. Hans setzt den Ofen.

Der Gärtner setzt Apfelbäume.

P1 Er setzt seine Hoffnung auf die Zukunft.

P1 Ich setze (fünfhundert Mark) auf dieses Pferd. Ich setze fünfhundert Mark darauf, daß dieses Pfierd gewinnt.

P1 Ich setze die Katze hinter den warmen ofen.

PO Das Pferd setzt über das Hindernis.

PO Es setzt Hiebe.

PO Der Kaffee setzt sich.

PO Der Rauch setzt sich in die Kleider.

Po Die Zuschauer setzen sich (auf die Stühle). Das Mädchen setzt sich mir auf den Schoß.

P1 Der Mechaniker setzt die alte Maschine instand.

P1 Die Regierung hat die Gmindrechte außer Kraft gesetit.

P1 'Der'Arbeiter setzt die Maschine in Betrieb.

P1 Der Zug setzt sich in Bewegung.

P1 Die Polizei setzt den Mord in enge Beziehung zu einer politischen Affäre.

P1 Spielende Kinder haben eine Scheune in Brand gesetzt. 
*in Erstaunen setzen

$\mathrm{T}$

*in Gang setzen

*in Kenntnis setzen über-Akk T

*in Kenntnis setzen von-Dat T

*in Kraft setzen

*in UmZauf setzen

*unter Druck setzen

*sich/Akk ins Benehmen setzen I mit-Dat

*sich/Akk in den Besitz setzen

\footnotetext{
*sich/Akk in Marsch setzen I

*sich/Akk in Trab setzen I

*sich/Akk ins Unrecht setzen I

mit-Dat

*sich/Akk in Verbindung setzen I. mit-Dat
}

P1 Deine ungewöhnliche Schweigsamkeit setzt mich in Erstaunen.

P1 Die Behörde setzt eine Untersuchung des Falls in Gang.

P1 Der Polizist setzte den Verdächtigen über seine Rechte in Kenntnis.

P1 Peter setzt seine Elterm von seinen Absichten in Kenntnis.

P1 Die Behörde setzt eine neue Dienstvorschrift in Kraft.

P1 Die Bundesbank setzt neue Geldscheine in Umlauf.

P1 Der Geschäftsmann setzt seinen Verhandlungspartner unter Druck.

PO Die Elterm setzen sich wegen der Tochter mit der Lehrerin ins Benehmen.

PO Der Spion hat sich in den Besitz geheimer Dokumente gesetzt.

Der Spion hat sich in den Besitz von geheimen Dokumenten gesetzt.

PO Die Kompanie setzt sich in Marsch.

PO Das Pferd setzt sich in Trab.

PO Mit dieser Lüge setzte sich Peter ins Unrecht.

PO Hans setzt sich mit seinem Freund in Verbindung. 
*sich/Akk zur Ruhe setzen

I

*sich/Akk zur Wehr setzen gegen - Akk

\section{singen}

singen

singen (von-Dat)

$0(4$

\section{sitzen}

sitzen

0

sitzen

05

Pertinenzelement im Dativ

sitzen

$0(5$

sitzen

$0(8$

\section{sparen}

sparen

01

sparen (an-Dat)

sparen (für-Akk)

\section{spielen}

spielen

spielen
PO Der alte Beomte setzt sich zur Ruhe.

PO Der Verfolgte setzte sich gegen die Polizisten zur Wehr.

P1 Das Mädchen singt (ein Lied).

P2 Der Junge singt (von der Liebe).

PO Der Hieb sitzt.

Po Paul sitzt auf dem Motorrad. Das Mädchen sitzt mir auf dem Schoß.

PO Paul sitzt (im Gefängnis).

PO Das neue Kleid sitzt (schlecht).

P1 Der alte Mann sparte viel Geld.

P2 Diese Fomilie spart (am Essen).

P2 Die Eltern sparen (für ein Auto).

PO Fritz spielt den Herrn.

P1 Der junge Mann spielt ein Konzert von Schumann. Der Schauspieier spielt den Faust. 


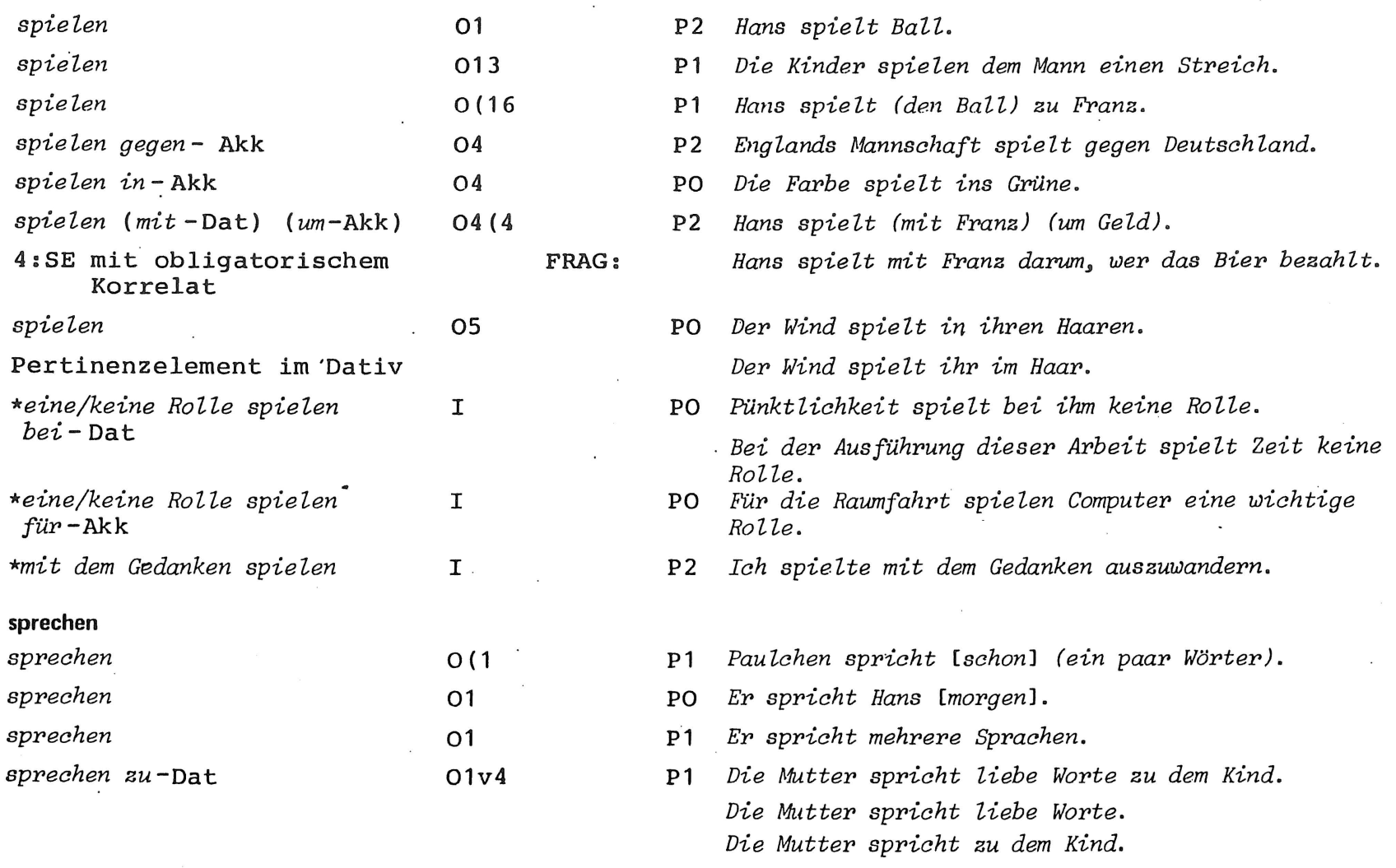


sprechen

$01 v 6$

sprechen aus-Dat

04

sprechen für-Akk

04

O:SE Zum Korrelat vgl.

$4: \mathrm{SE}$ mit obligatorischem Korrelat

sprechen gegen - Akk

O:SE Zum Korrelat vgl. Einleitung 6.3.5.

4:SE mit obligatorischem Korrelat

sprechen mit-Dat über-Akk

04

04v 4
P2 Er spricht einige Worte ins Mikrophon.

Er spricht einige Worte.

Er spricht ins Mikrophon.

Po Zärtlichkeit spricht aus seinen Augen. Er spricht aus Erfahrung.

PO Dein Verhalten spricht für dich.

DASS : Es spricht für ihre Liebe, daß sie dies getan hat.

INF+: $\quad$ Alles spricht dafür, jetzt mit der Arbeit Schluß zu machen.

DASS : Ihr Verhalten spricht dafür, daß sie intelligent ist.

PO Diese Tatsache spricht gegen dich.

DASS: Es spricht gegen dich, daß du so wenig arbeitest.

INF+:

DASS :
Alles spricht dagegen, jetzt mit der Arbeit anzufangen.

Alles spricht dagegen, daß wir jetzt mit der Arbeit anfangen.

P2 Sie spricht mit ihrem Mann über die neue Waschmaschine.

Sie spricht mit ihrem Kollegen.

Sie spricht über ihren Urlaub in Griechenland. 


\section{4:SE mit obligatorischem} Korrelat

sprechen mit-Dat von-Dat

$04 v 4$

4:SE mit obligatorischem Korrelat

INF+ :

DASS :

FRAG :

sprechen (über - Akk) (zu-Dat)

O $(4) 4$

4:SE mit obligatorischem Korrelat

DASS :

FRAG :

\section{springen}

springen

0

springen

01

springen

06

Pertinenzelement im Dativ

\section{starten}

starten

BEMERKUNG :
DASS :

FRAG :

Sie spricht mit ihrem Mann darüber, daß sie eine neue Waschmaschine kaufen will.

Sie spricht mit ihm darüber, wie die neue Maschine aussehen soll.

P2 Er sprach mit uns von seinen Zielen.

Er sprach mit uns.

Er sprach von seinen Zielen.

Er sprach davon, in die Ferien zu fahren.

Er sprach davon, daß er Beanter werden wolle.

Er sprach davon, wie sehr er sie verachtet.

P2 Der Minister spricht (zu den Abgeordneten) (über die Steuererhöhung).

Der Minister sprach zu den Abgeordneten darüber, daß Steuererhöhungen vermieden werden sollten.

Der Minister sprach zu den Abgeordneten nicht darüber, ob er die Steuern erhöhen wollte oder nicht.

PO Das Glas springt.

P1 Bob Beamon sprang 8,90 m.

PO Der Löwe springt durch den Reifen.

Der Hund springt ihm an den Hals.

P1 Der Fahrer startet den Wagen.

STAMMFORMEN : starten, startete, hat gestartet. 


\section{starten}

starten

BEMERRUNG:

$O(5) 6$

\section{stattfinden}

stattfinden

stecken

stecken

stecken

016

Pertinenzelement im Dativ

stecken

Pertinenzelement im Dativ

\section{stehen}

stehen

stehen

stehen nach-Dat

4:SE mit obligatorischem Korrelat

stehen auf-Akk

stehen auf-Akk
05

0

03

034

INF+ :

04

04
PO Das Flugzeug startet (um acht) (von Frankfurt). STAMMFORMEN : starten, startete, ist gestartet.

PO Der Empfang findet (am Sonntag um zwölf Uhr) statt.

P1 Der Bauer steckt Rüben.

P1 Er steckt den Brief in den Umschlag. Der Mann steckt seiner Freundin den Ring an den Finger.

PO Der Wagen steckt im Schlomm. Mir steckt eine Gräte im Hals.

Po Die Uhr steht.

PO Das Kleid steht ihr [gut].

Po Paul steht der Sinn nach gutem Essen. Ihm steht der Sinn danach, eine Bergwanderung zu machen.

Po Auf Mord steht die Todesstrafe.

PO Er steht ouf blonde Mädchen. 


\section{4:SE mit obligatorischem Korrelat}

stehen zu-Dat

04

stehen

05

Pertinenzelement im Dativ

stehen

stehen

06

*Modell stehen

*Pate stehen bei-Dat

$\star$ Posten stehen

*Schlange stehen

*seinen Mann stehen

*Wache stehen

*auf gutem Fuße stehen mit-Dat I

*auf dem spiel stehen

*in Aussicht stehen

*in Blüte stehen

*in Frage stehen

*im Gegensatz stehen zu-Dat

*in Kontakt stehen mit-Dat

«im Ruf stehen
INF+:

Ich stehe nicht darauf, heute ins Kino zu gehen.

PO Ich stehe zu meinem Wort.

PO In Mannheim steht ein Schloß.

Er steht mir ouf dem Fuß.

PO Seine FüBe stehen nach innen.

PO Die Tür steht offen.

P2 Das Kind steht Modell.

Po Er steht bei seinem Neffen Pate.

Po Der Soldat steht Posten.

P2 Die Leute stehen vor dem Kino Schlange.

PO Dieter steht seinen Mann.

P2 Der Soldat steht Wache.

PO Sie steht mit ihren Eltern ouf gutem Fuße.

Po Die Freiheit der Berufswahl steht auf dem Spiel.

Po Für Herrn Meier steht eine Beförderung in Aussicht.

PO Der Apfelbaum stand in voller Blüte.

PO Der Wert deiner Arbeit steht nicht in Frage.

- $\quad$ PO Deine Rede steht im Gegensatz zu früheren Äußerungen.

po Wir stehen mit unseren Nachbarn in engem Kontakt.

PO Er steht im Ruf, ein Trinker zu sein. 
* in Verbindung stehen mit-Dat I

*in Widerspruch stehen zu-Dat I

*im Zuscommenhang stehen mit-Dat I

*unter Aufsicht stehen I

*unter Druck stehen I

*unter Eid stehen I

*unter Kontrolle stèhen I

*zur Debatte stehen

*zu Diensten stehen

*zur Diskussion stehen

*zur Seite stehen

*zur Verfügung stehen

stehlen

stehlen

steigen

siteigen
PO Er steht mit seinen Klassenkameraden noch in Verbindung.

PO Seine Erkenntnisse stehen im Widerspruch zur gescomten bisherigen Forschung.

P.O Unser Entschluß steht in engem Zuscomenhang mit deiner Empfehlung.

PO Die Kinder stehen ständig unter ärztlicher Aufsicht.

PO Der Lehrling steht unter starkem Druck.

PO Der Zeuge steht unter Eid.

PO Dieses Produkt steht unter der ständigen Kontrolle eines Lebensmittelchemikers.

Po Dein Vorschlag steht zur Debatte.

PO Ich stehe gern zu ihren Diensten.

Po Der Plan steht zur Diskussion.

PO Michael stand seinem Freund hilfreich zur Seite.

PO Ich werde dir zur Verfügung stehen.

P1 Die Diebe haben ein wertvolles Gemälde gestohlen.

PO Die Preise steigen.

Das Wasser steigt.

Die Straße steigt. 
steigen in-Dat

04

steigen

06

Pertinenzelement im Dativ steigen

$0(6$

stellen

stelzen

stellen

stellen (auf-Akk)

01

013

$01(4$

stellen

016

stellen

sich/Akk stellen

sich/Akk stelzen

sich/Akk stellen mit-Dat

sich/Akk stellen

Pertinenzelement im Dativ

*einen Antrag stellen auf-Akk

*einen Antrag stellen bei-Dat I

* ein Bein stellen

018

03

013

048

06

*auf die Probe stellen

*in Abrede stellen
PO Alze Waren sind im Preis gestiegen.

PO Der Großvater steigt auf den Donnersberg. Die Tränen steigen ihr in die Augen.

Po Das Thermometer steigt (auf zehn Grad).

P1 Die Polizei stellt den Dieb.

P1 Der Lehrer stellt den Schulern eine schwere Aufgabe.

P1 Die Mutter stellt ihren Wecker (auf füf Uhr).

P1 Das Kind stellt den Topf in den Schrank.

P1 Der Kellner stellt den Wein kalt.

PO Die Politiker stellen sich den Fragen der Wähler.

PO Der Dieb stellt sich (der Polizei).

PO Er hat sich mit seinen Vorgesetzten gut gestellt.

PO Er hat sich dem Auto in den Weg gestelzt. Er hat sich mir an die Seite gestellt.

P1 Wir stellen einen neuen Antrag ouf Verlängerung des Projekts.

P1 Er stelzt einen Antrag bei der Behörde.

PO Franz stellt seinem Freund ein Bein.

P1 Er stellt seinen Freund auf die Probe.

P1 Hans stellt seine Schuld in Abrede. 
*in Aussicht stelzen

*in Frage stellen

*in Rechnung stelzen

*unter Beweis stellen

*unter Strafe stellen

*zur Debatte stellen

*zur Diskussion stellen

*zur Rede stellen

*zur Schau stellen

*zur Verfügung stellen

*zur Wahl stellen

\section{sterben}

sterben

sterben

\section{stimmen}

stimmen
P1 Das Ministerium hat dem Beanten eine Beforderung in Aussicht gestellt.

P1 Der Verteidiger stellt die Aussage des Zeugen in Frage.

P1 Er stellte ihre Einwände in Rechnung.

P1 Der Abgeordnete stellte seine Unbestechlichkeit unter Beweis.

P1 Der Gesetzgeber hat den Handel mit Rauschgift unter Strafe gestellt.

P1 Der Minister stellt das Problem erneut zur Debatte.

P1 Der Abgeordnete stellt seinen Vorschlag zur Diskussion.

P1 Der Sohn stellt seinen Vater zur Rede.

P1 Der junge Mann stellt sein Wissen zur Schau.

P1 Er stellt seinem Bruder sein Auto zur Verfügung.

P1 Alle vier Jahre stellen die Parteien ihre Kandidaten zur Wahl.

Po Der Verletate ist [auf dem Weg ins Krankenhaus] gestorben.

Po Er stirbt einen schweren Tod.

PO Deine Aufgaben stimmen. 


\section{O:SE Zum Korrelat vgl. Einleitung 6.3.5.}

stimmen

01

stimmen

018

O:SE Zum Korrelat vgl. Einleitung 6.3.5.

stimmen für-Akk

4:SE mit obligatorischem Korrelat

stimmen gegen - Akk

4:SE mit obligatorischem Korrelat

\section{stören}

stören

stören (bei-Dat)

O:SE Zum Korrelat vgl. Einleitung 6.3.5.

stören (in - Dat)

04

04

$0(1$
DĀSS :

Es stimmt nicht, daß sein Bruder reich war.

P1 Der Musiker stimnt sein Instrument.

P1 Das Geschenk des Ehemannes stimmt die Frau fröhlich.

INF+:

DASS :

INF+:

DASS :

INF+:

DASS :

$0(1)$

INF+:

DASS :

$0(1(4$ Es stimmt mich glücklich, dich bei mir zu haben.

Es stimnt den jungen Mann traurig, daß das Mädchen ihn nicht liebt.

P2 Wir stimmen für diesen Kandidaten.

Die Opposition hat dafür gestimmt, dieses Gesetz aufzuheben.

Ich stimme dafür, daß der Antrag angenommen wird.

P2 Die Schüler stimmen gegen den Vorschlag des Lehrers. Ich stimme dagegen, diesen Vorschlag anzunehmen. Ich stimme dagegen, daß dieser Vorschlag angenommen wird.

P1 Das Lärmen der Kinder stört (den Vater).

P1 Dieser Lärm stört (mich) (bei der Arbeit).

Es stört mich beim Lesen, die laute Radiomusik meines Nachbarn hören zu müssen.

Es stört mich bei meiner Arbeit, daß die Kinder so Zärmen.

P1 Das Kindergeschrei stört (mich) (in meiner Arbeit). 


\section{O:SE Zum Korrelat vgl. Einleitung 6.3.5.}

sich/Akk stören an-Dat

\section{4:SE mit obligatorischem} Korrelat

\section{stoßen}

stoßen

stoßen

sich/Akk stoßen an-Dat

4:SE mit obligatorischem Korrelat

BEMERKUNG :

\section{stoßen}

stoßen an-Akk

stoßen auf - Akk

04

stoßen

06

INF+:

DASS :

04

$01(6$

04

BEMERKUNG :
Es stört mich in meiner Arbeit, dir jetat deine Fragen beantworten zu müssen.

Es stört mich in meiner Ruhe, daß die Kinder so laut sind.

PO Der Meister stört sich an den langen Haaren des Lehrlings.

INF+: Der Lehrer stört sich daran, von den Schülern nicht gegrüßt zu werden.

DASS: $\quad$ Der Lehrer stört sich daran, daß ihn die Schüler nicht grïßen.

P1 Der Lehrer stößt seinen Freund [om Arm]. Er hat einen neuen Rekord gestoßen.

P1 Ich habe meinen Bruder (vom Stuhl) gestoßen.

Po Viele Leute stoßen sich an den langen Haaren der Jugendlichen.

DASS: $\quad$ Manche Leute stoßen sich daran, daß viele JugendZiche lange Haare tragen.

STAMMFORMEN: stoßen, stieß, hat gestoßen.

PO Das Haus stößt direkt an das benachbarte Grundstück.

PO Ich bin in diesem Buch ouf viele Fehler gestoßen.

PO Er ist [im Dunkeln] gegen die Stehlampe gestoßen. STAMMFORMEN: stoßen, stieß, ist gestoßen. 


\section{streiten}

streiten für-Akk

04

4:SE mit obligatorischem Korrelat

<sich/Akk > streiten mit-Dat ( uber - Akk)

4:SE mit fakultativem Korrelat

<sich/Akk > streiten mit-Dat ( um - Akk)

4:SE mit obligatorischem Korrelat

\section{studieren}

studieren

studieren

$0(1$

\section{stürzen}

sturzen

01

stürzen

sich/Akk stürzen auf-Akk

016

04

4:SE mit obligatorischem Korrelat
P2 Der junge Mann streitet für seine Idee.

DASS: $\quad$ Der junge Mann streitet dafür, daß seine Idee verwirklicht wird.

PO Der Lehrer streitet <sich> mit einem Kollegen (ïber ein Problem).

FRAG: Der Lehrer hat sich mit einem Kollegen <darüber > gestritten, was dieses Wort bedeutet.

Ich habe <mich> mit einem anderen Autofahrer (um einen Parkplatz) gestritten.

INF+ :

Ich habe mich mit meinem Bruder darum gestritten, sein Rad benutzen zu dürfen.

FRAG: Er hat sich mit seiner Schwester darum gestritten, wer mit Vater in die Stadt fahren darf.

P1 Der Reisende studiert den Fahrplan.

P1 Ihr Freund studiert (Geschichte).

P1 Das Volk stürzt die Regiemung.

P1 Er stürat ihn ins Unglück.

Po Die Kinder stürzten sich ouf das Spiel.

INF+: $\quad$ Die Kinder stürzten sich darauf, die Aufgaben zu Zösen. 
sich/Akk stilrzen

06

Pertinenzelement im Dativ BEMERKUNG :

\section{stürzen}

stürzen

stürzen

06

016

BEMERKUNG :

\section{suchen}

suchen

suchen nach-Dat
PO Der Mann stürat sich ins Wasser.

Sie stürzt sich ihm zu Füßen.

STAMMFORMEN: stürzen, stürzte, hat gestürzt.

PO Mein Freund stürzte ins Zimmer.

PO Das Pferd stürzte (zu Boden).

STAMMFORMEN: stürzen, stürzte, ist gestürzt.

P1 Die Kinder suchen Blumen.

Der Kranke sucht Hilfe.

P2 Der Mann sucht nach seinem Kugelschreiber. 


\section{tanken}

tanken

$0(1$

\section{tanzen}

tanzen

O 1

tanzen

06

teilen

teilen

teilen mit-Dat

01

teilen (in - Akk)

014

teilen (unter - Akk)

sich/Akk teilen in-Akk

sich/Akk teilen (in-Akk)

<sich/Dat> teilen

\section{teilnehmen}

teilnehmen an-Dat

\section{telefonieren}

telefonieren ( mit-Dat)

telefonieren
P1 Der Autofahrer tankte (20 I Benzin).

P1 Die Gäste tanzen (Tango).

P2 Sie tanzen durch den Saal.

P1 Er teizt meine Meinung.

P1 Mein Freund teilt seine Sorgen mit mir.

P1 Die Mutter teilt den Kuchen (in sechs Stücke).

P1 Die Mutter teizt den Kuchen (unter die sechs Kinder).

PO Die Parteien teilen sich in die Prozeßkosten.

PO Der Fluß teilt sich (in drei Arme).

P1 Die Chefs teilten <sich> die Arbeit.

P2 Seine Freundin nahm an unserem Fest teil.

P2 Der Angestellte telefoniert (mit seiner Frau).

P2 Ich habe [gestern] (nach Hamburg) telefoniert. 


\section{töten}

töten

01

\section{tragen}

tragen

tragen

tragen

$01(6$

tragen

1:SE Zum Korrelat vgl. Einleitung 6.3.5.

tragen an-Dat

4:SE mit obligatorischem Korrelat

tragen

*Bedenken tragen

* Rechnung tragen

*zur Schau tragen

*sich/Akk mit einem Gedanken tragen

048
P1 Der Verbrecher hat eine junge Frau getötet. Der Diktator hat sich [selbst] getötet.

P1 Das Mädchen trägt einen Hut.

Das Kapital trägt Zinsen.

po Der Baum trägt (viele Apfel).

Das Eis trägt [schon] (einen erwachsenen Mann).

P1 Die Mutter trägt das Kind (zum Arzt).

P1 Sie trägt das Haar lang.

INF+: $\quad$ Er trägt es mit Geduld, unheilbar krank zu sein.

DASS: $\quad$ Er trägt es mit Geduld, daß seine Frau stundenlang telefoniert.

PO Er trägt schwer an seinem Unglück.

INF+: $\quad$ Er trägt schwer daran, krank zu sein.

DASS: $\quad$ Er trägt schwer daran, daß er im Bett liegen muß.

PO Der Baum trägt gut.

PO Ich trage keine Bedenken, die Kinder auf der Straße spielen zu Zassen.

P2 Ich werde deinem Vorschlag Rechnung tragen.

P1 Er trägt eine finstere Miene zur Schau.

Po Ich trage mich mit dem Gedanken, nach Kanada ouszuwanderm. 


\section{treffen}

treffen

treffen

01

01

treffen

treffen (mit-Dat)

$01(4$

treffen

$01(5$

treffen

$01(8$

O:SE Zum Korrelat vg'l. Einleitung 6.3 .5 .

treffen

Pertinenzelement im

Akkusativ

sich/Akk treffen (mit-Dat)

BEMERKUNG :

\section{treffen}

treffen auf-Akk (mit-Dat)

$04(4$

BEMERKUNG :

06

$0(4$
PO Den Mann trifft keine Schuld.

P1 Ich habe meine Wahl getroffen.

Ich treffe eine Entscheidung.

Er trifft Vorbereitungen für seine UrZaubsreise.

P1 Der Stein traf (das Fenster).

P1 Hans trifft das Fenster (mit dem Stein).

PO Ich habe deinen Freund (auf der Straße) getroffen.

P1 Der Tod seiner Eltern hat ihn (schwer) getroffen.

INF+: $\quad$ Es hat mich tief getroffen, ihn krank au sehen.

DASS :

Es hat ihn tief getroffen, daß seine Eltern gestorben sind.

Po Die Kugel traf ins Schwarze.

Das Messer traf ihn ins Herz.

PO [Morgen] treffen wir uns (mit unseren Freunden). STAMMFORMEN : treffen, traf, hat getroffen.

Po Sie traf (mit ihrem Vorschlag) auf Schwierigkeiten. STAMMFORMEN: treffen, traf, ist getroffen 


\section{trennen}

trennen

01

trennen aus - Dat

014

trennen von-Dat

014

trennen (von-Dat)

01 ( 4

sich/Akk trennen von-Dat

\section{treten}

treten

treten in-Akk

treten

Pertinenzelement im Akkusativ

Pertinenzelement im Dativ

zutagetreten

O

O:SE Zum Korrelat vgl. Einleitung 6.3.5.
P1 Du hast dieses Wort [falsch] getrennt. Das Fernsprechamt trennt das Gespräch.

P1 Die Mutter trennt den Reißverschluß aus dem Kleid.

P1 Die Hausfrau trennt das Fleisch von den Knochen.

P1 Die Mutter trennt die beiden streitenden Kinder (voneinander).

Po Der Ehemann trennte sich von seiner Frau.

P1 Die Leute treten einen Pfad.

Der Gitarrist tritt den Takt.

PO Sie treten in den Stand der Ehe.

Er tritt in den Ruhestand.

PO Ich trete auf die Straße.

Er tritt mich in den Hintern.

Er tritt mir auf den Fuß.

PO Das Problem tritt zutage.

DASS : 
*in Beziehung treten mit-Dat

*in Beziehung treten zu- Dat

*in Erscheinung treten

I

*in Kraft treten

I

\section{trinken}

trinken

trinken

018

trinken auf-Akk

4:SE mit obligatorischem Korrelat

trinken (von - Dat)

$\star$ Brüderschaft trinken mit - Dat

tun

tun

Pertinenzelement im Dativ tun

sich/Akk tun

O
P2 Er tritt mit seinen Nachbarn in nähere Beziehung.

P2 EY ist zu seinem Geschäftspartner noch nicht in private Beziehung getreten.

PO Der Held tritt erst an Schluß des Stückes in Erscheinung.

PO Der Vertrag tritt morgen in Kraft.

P1 Das Baby trinkt (Milch).

P1 Er trinkt sein Glas leer.

P2 Er trinkt auf meine Gesundheit.

DASS: Sie trinken darauf, daß der plan gelingt.

P2 Das Baby trinkt (von der Milch).

po Sebastian trank mit allen Brüderschaft.

P1 Er tut nichts.

Er tut mir einen Gefallen.

Po Er tut wichtig.

PO [Auf der Maimesse] tut sich etwas.

$\mathrm{E}_{\mathrm{O}}$ meist indefinit. 
wehtun

$\mathrm{O}$

Pertinenzelement im Dativ

wehtun

03

O:SE Zum Korrelat vgl. Einleitung 6.3.5.

\section{turnen}

turnen

turnen (an-Dat)
O 11

$O(4$
PO Der Kopf tut mir weh. Mir tun die Fuße weh.

P2 Du hast deinem Freund wehgetan. Es tut mir weh, die Not unserer Nachbarm mit ansehen zu müssen.

Es tut mir weh, daß du so traurig bist.

P1 Hans turnt (seine Bodenibung).

P2 Helga turnt (am Reck). 


\section{üben}

üben

üben

sich/Akk üben (in - Dat)

4:SE mit obligatorischem Korrelat

überfahren

überfahren

\section{überholen}

uberholen

überholen

\section{überlegen}

überlegen

1:SE mit fakultativem Korrelat

\section{BEMERKUNG :}

sich/Dat überlegen

$1: S E$ mit fakultativem Korrelat

01

01
O ( 1

$0(4$

INF+ : Das Kind übt sich darin, Briefe zu schreiben.

P1 Mein Freund hat eine Katze überfahren. Der Zug hat ein Haltesignal überfahren.

P1 Er hat [an seinem Freund] Verrat geübt.

P1 Die Kinder üben (ein Lied).

PO Das Kind übt sich (im Schreiben).

P1 Der Mechaniker überholt das Auto.

P1 Das Auto überholt (den Lastwagen).

PO Ich überlege [gerade] (etwas).

Ich überlege <es> mir gründlich, ob ich mit dir ins Kino gehe.

$E_{1}$ meist indefinit oder SE.

Wir überlegen 〈es> uns, ein Haus zu kaufen.

Wir haben <es> uns gerade überlegt, daß wir heute abend tanzen gehen könnten. 


\section{1:SE mit fakultativem} Korrelat

\section{übernachten}

übernachten

\section{überqueren}

überqueren

\section{überraschen}

überraschen

O:SE Zum Korrelat vgl.

überraschen (bei-Dat)

überraschen (mit-Dat)

\section{4:SE mit obligatorischem} Korrelat

\section{überreden}

überreden ( $z u-D a t)$

\section{4:SE mit fakultativem} Korrelat

FRAG :

Wir haben <es> uns überlegt, ob wir nicht schon heute wegfahren sollten.

P2 Meine Freundin übermachtet im Hotel.

P1 Der Fußgänger überquert die Straße [bei einer Verkehrsampe[].

O(1)

$\mathrm{INF}+$ :

DASS :

$01(4$

$01(4$

DASS :
P1 Dein schlechtes Zeugnis überrascht (uns) [nicht]. Es ïberrascht mich sehr, dich hier zu sehen. Es hat uns kaum überrascht, daß du gestern nicht gekommen bist.

P1 Die Mutter überrascht ihren Sohn (beim Rauchen).

P1 Der Arzt hat mich (mit einer schlimmen Nachricht) überrascht.

Der Angestellte hat den Chef domit überrascht, daß er zum nächsten Ersten gekündigt hat.

P1 Der junge Mann überredet seine Frewndin (zu einem Kinobesuch).

Der Mann überredet seine Frau <dazu>, die schwarzen schuhe zu kaufen. 


\section{4:SE mit fakultativem} Korrelat

HPTS :

\section{übersetzen}

$$
\begin{aligned}
& \text { übersetzen (ous-Dat) } \\
& \text { (in-Akk) } \\
& \text { übersetzen (von-Dat) }
\end{aligned}
$$$$
\text { (in-Akk) }
$$

\section{überweisen}

uberweisen

$01(3$

überweisen

$01(6$

\section{überzeugen \\ überzeugen (von-Dat) \\ $4: \mathrm{SE}$ mit obligatorischem Korrelat}

$01(4$

sich/Akk überzeugen von-Dat

04

4:SE mit fakultativem Korrelat

\section{umsteigen}

umsteigen auf-Akk

umsteigen

DASS :
Der Mann überredet seine Frau <dazu>, sie solle die schwarzen Schuhe kaufen.

P1 Wir übersetzen einen Text (aus dem Englischen) (ins Deutsche).

P1 Wir übersetzen einen Text (vom Englischen) (ins Deutsche).

P1 Er überweist (seinem Sohn) einen Mietzuschuß.

P1 Herr Bauer überweist den Lohn (an seine Angestelzten).

P1 Der Politiker überzeugt die Leute (von seinem Prograrm). INF+: $\quad$ Er überzeugt die Leute davon, das richtige Program zu haben.

Er überzeugt die Leute davon, daß er das richtige Progromm hat.

PO Franz überzeugt sich von der Qualität des Anzugstoffes.

DASS : $\quad$ Er ïberzeugt sich <davon>, daß sein neuer Anzug gut sitzt.

P2 Der Angestellte steigt auf einen anderen Beruf um.

P2 Die Reisenden steigen (in einen anderen Zug) um. 


\section{umziehen}

umziehen

01

\section{BEMERKUNG :}

\section{umziehen}

umziehen

BEMERKUNG :

\section{unterbrechen}

unterbrechen

\section{unterhalten}

unterhalten

unterhalten (mit-Dat)

sich/Akk unterhalten (mit-Dat) o(4) 4 ( iber-Akk)

$4: \mathrm{SE}$ mit obligatorischem Korrelat
P1 Die Mutter zieht ihr Kind um.

Isolde zieht sich um.

STAMMFORMEN : umziehen, zog um, hat umgezogen.

P2 Die Familie zieht (nach Mannheim) um.

STAMMFORMEN : umziehen, zog um, ist umgezogen.

P1 Der Anruf unterbrach den Arzt [bei der Untersuchung]. Der Besuch unterbrach unser Gespräch.

P1 Die Gewerkschaft unterhält [hier] ein Erholungsheim.

P1 Die Gastgeberin unterhält ihre Gäste (mit einem Lied).

PO Die Gäste unterhalten sich (mit uns) (über das Konzert).

DASS :

Die Kinder unterhalten sich mit den Eltern darüber, daß sie im Kino einen interessanten Film gesehen haben.

FRAG: Die Kinder unterhalten sich mit den Elterm darüber, . ob sie gemeinsom in Urlaub fahren sollen. 


\section{unterrichten}

unterrichten (in-Dat)

unterrichten (über-Akk)

$01(4$

$01(4$

4:SE mit obligatorischem Korrelat

unterrichten (von-Dat)

4:SE mit obligatorischem Korrelat

DASS :

FRAG :

\section{unterschreiben}

unterschreiben

\section{untersuchen}

untersuchen

1:SE mit fakultativem Korrelat

untersuchen ( auf-Akk)

4:SE mit obligatorischem Korrelat

DASS :

01

FRAG
P1 Der Lehrer unterrichtet die Kinder (in Deutsch).

P1 Der. Ingenieur unterrichtet die Arbeiter (über die neuen Sicherheitsvorschriften).

Er unterrichtet seine Versicherung darïber, daß er einen Autounfall hatte.

Er unterrichtet seine Versicherung darüber, wann sich der Unfall ereignet hat.

P1 Sie hat uns (von ihren Plönen) unterrichtet.

Ich habe die Polizei davon unterrichtet, daß mein Auto gestohlen ist.

Ich habe die Polizei davon unterrichtet, wo mein Auto gestohlen wurde.

P1 Der Mieter unterschreibt (den Mietvertrag).

P1 Die Polizei untersucht einen schwierigen Fall. Die Polizei untersucht <es> sorgfältig, ob es sich hier um einen Unfall handelt.

P1 Der Gerichtsmediziner untersucht die Kleidung (auf Blutflecken).

FRAG:Der Arst untersucht Sepp darauf, ob sein Herz gesund ist. 


\section{verabreden}

verabreden mit - Dat

014

$1: \mathrm{SE}$ mit fakultativem Korrelat

sich/Akk verabreden (mit - Dat) o(4 (4 ( $z u$ - Dat)

\section{verabschieden}

verabschieden

01

sich/Akk verabschieden

(von-Dat)

O 14

\section{verachten}

verachten

1:SE Zum Korrelat vgl. Einleitung 6.3.5.

\section{verändern}

verändern

01

sich/Akk verändern
P1 Gerd verabredet mit Carola den nächsten Termin.
INF+ :

DASS :

FRAG :

HPTS : 


\section{verbessern}

verbessern

O:SE Zum Korrelat vgl. Einleitung 6.3.5.

sich/Akk verbessern

01

DASS :

0

\section{verbieten}

verbieten

verbieten

$1: S E$ mit fakultativem Korrelat

\section{verbinden}

verbinden

Pertinenzelement im Dativ

verbinden (mit-Dat)

verbinden (mit-Dat)

O:SE Zum Korrelat vgl. Einleitung 6.3.5.
P1 Der Schüler verbessert seine Arbeit.

Es hat die Lage des Studenten verbessert, daß er einen Scheck bekommen hat.

PO Schmidt hat sich [im Schachspieien] verbessert.

P1 Das neue Gesetz verbietet die Spielkasinos.

P1 Seine Frau verbietet ihm die Zigaretten.

INF+: Seine Frau verbietet <es > ihm zu rouchen.

P1 Der Arzt verbindet meinen Arm.

Der Arzt verbindet mir den Arm.

P1 Der Gang verbindet die Küche und das Zimmer (miteinander).

Der Gang verbindet die Küche mit dem Zimmer.

P1 Gemeinsame Interessen verbinden die beiden Freunde (miteinander).

INF+: $\quad$ Es verbindet uns miteinander, den gleichen Beruf zu haben.

Es verbindet uns, daß wir die gleichen Interessen haben. 


\section{verdienen}

verdienen

01

O:SE Zum Korrelat vgl. Einleitung 6.3.5.

1:SE mit fakultativem Korrelat

verdienen

verdienen an-Dat

4:SE mit obligatorischem Korrelat

verdienen

\section{vergessen}

vergessen

$1: \mathrm{SE}$ mit fakultativem Korrelat

01
DASS :

INF+:

DASS :

sich/Akk vergessen
PO Diese Meinung verdient Achtung.

Es verdient unsere Achtung, daß du jeden Morgen pünktlich kommst.

Johann verdient $\langle$ es $\rangle$, geachtet zu werden.

Johann verdient $\langle e s\rangle, d a \beta$ er geachtet wird.

P1 Mein Vater verdient viel Geld.

P2 Die Firma verdient [schon immer] an mir.

Die Firma verdient gut daran, daß ich bei ihr arbeite.

P2 Mein Vater verdient gut.

P1 Der Chef vergaß einen wichtigen Termin.

INF+: $\quad$ Der Chef vergaß <es > oft, einen wichtigen Termin einzuhazten.

DASS: $\quad$ Der Chef vergaß<es>, daß er einen wichtigen Termin hatte.

FRAG: Der Chef hatte <es> völlig vergessen, ob er noch einen wichtigen Termin hatte.

PO Der Polizist vergaß sich. 


\section{vergleichen}

vergleichen (mit-Dat)

sich/Akk vergleichen mit-Dat

\section{verhaften}

verhaften

\section{verhalten}

verhalten

sich/Akk verhalten

sich/Akk verhalten (es) mit-Dat

\section{verkaufen}

verkaufen

$01(3$ ( 8

verkaufen (an - Akk)

$01(4) 8$

verkaufen

$01(6(8$

sich/Akk verkaufen

* für dumm verkaufen
P1 Der junge Ehemann vergleicht seine Frau und seine Mutter miteinander.

Er vergleicht seine Frau mit seiner Mutter.

Er vergleicht sich mit seinem Bruder.

PO Firma $A$ hat sich mit Firma $B$ verglichen.

P1 Die Polizei hat den Verdächtigen verhaftet.

Po Die Kinder verhielten den Atem.

PO Hans verhält sich ruhig.

Die Sache verhält sich anders.

PO Mit meiner Gesundheit verhält es sich schlecht.

P1 Der Bauer verkauft (den Nachbarn) die Eier (billig).

P1 Der Bauer verkauft sein Land (an den Staat) (zu einem günstigen Preis).

P1 Ich verkaufe Fernsehapparate (ins Ausland) (mit hohen Gewinnen).

PO Er hat sich gut verkauft.

P1 Du kannst mich nicht für dumm verkaufen. 


\section{verlangen}

verlangen (von-Dat)

$01(4$

1:SE ohne Korrelat

verlangen nach - Dat

$4: S E$ mit obligatorischem Korrelat

\section{verlängern}

verlängern

verlängern (ouf-Akk)

verlängern (um-Akk)

\section{verlassen}

verlassen

sich/Akk verlassen ouf-Akk

4:SE mit obligatorischem Korrelat

04

$01(4$

$01(4$

01

04
P1 Der Kunde verlangt ein Kilo Äpfel (vom Kaufmann). INF+: Hans verlangt, den Chef zu sprechen.

DASS : Hans verlangt, daß er den Chef sprechen kann.

P2 Das Kind verlangt nach der Mutter.

INF+: $\quad$ Das kranke Kind verlangt danach, seine Mutter zu sehen.

DASS: Das Kind verlangt danach, daß die Mutter kommt.

$01 \cdot \cdot$

P1 Die Mutter verlängert die Kleider.

P1 Sie veriängern den Vertrag (auf fünf Jahre).

P1 Sie verlängern den Vertrag (um fünf Jahre).

P1 Er verließB Eltern und Freunde.

Das Glück verläßt ihn.

PO Ich verlasse mich auf seinen Rat.

INF+: Der Fluggast verläßt sich darauf, sicher anzukommen.

DASS :
Der Fluggast verläßt sich darauf, daß er sicher Zanden wird. 


\section{verletzen}

verletzen

Pertinenzelement im Dativ

\section{verlieren}

verlieren

veritieren

verlieren (an-Akk)

verlieren an-Dat

sich/Akk verlieren

sich/Akk verlieren

*aus den Augen verlieren

\section{vermieten}

vermieten

vermieten an-Akk
$01(4$

0

05

$01(3$

$01 \mathrm{v} 4$
P1 Der Verbrecher hat das Gesetz verletzt. Ich habe mir den Arm verletzt.

P1 Die Firma verliert ihren besten Angestellten.

P1 Hans hat hundert Mark verZoren.

Der Motor verliert $8 Z$.

Ich habe das Spiel verloren.

P1 Ich habe [gestern] hundert Mark (an meinen Freund) verloren.

Po Der Kranke verliert an Gewicht.

PO Mein Lampenfieber hat sich verloren.

PO Das Kind verlor sich in der Menge.

P1 Rudolf hat sein liel nicht aus den Augen verloren.

P1 Herr Müller vermietet (dem Studenten) ein Zimmer.

P1 Frau Meyer vermietet ihr Zimmer an berufstätige Damen.

Frau Meyer vermietet Zimmer.

Frau Meyer vermietet [nur] an berufstätige Damen. 


\section{vermuten}

vermuten

1: SE ohne Korrelat

$1:$ SE mit fakultativem Korrelat

\section{BEMERKUNG :}

vermuten

\section{veröffentlichen}

veröffentzichen

veröffentzichen

\section{verreisen}

verreisen

\section{verschreiben}

verschreiben

verschreiben
015

$01(5$

01

INF+:

FRAG :

HPTS :

DASS :

15

$01(3$
P1 Der Arzt vermutet eine schlimme Krankheit. Der Patient vermutet, eine schlimme Krankheit zu haben. Hans vermutet, wo seine Frau sein könne.

Der Briefträger vermutet, Frau Schmitt ist nicht zu Hause.

Der Lehrer hat <es > schon lange vermutet, daß dieser Schüler wenig lernt.

$\mathrm{E}_{1}$ meist indefinit oder $\mathrm{SE}$.

P1 Die Dame vermitet einen Dieb in ihrer Wohnung.

P1 Der Minister veröffentlicht seine Dokumentation [auf einer Pressekonferenz].

P1 Er veröffentlicht seine Forschungsergebnisse [meistens] (in Zeitschriften).

P2 Ich verreise [morgen].

P1 Er hat [bei seiner Arbeit] einen ganzen Bleistift verschrieben.

P1 Der Arzt verschreibt (ihm) Tabletten und acht Tage Ruhe. 
sich/Akk verschreiben

O

sich/Akk verschreiben

03

\section{versichern}

versichern

012

versichem

1:SE mit fakultativem Korrelat

0113

INF+ :

DASS :

HPTS :

\section{BEMERKUNG :}

versichern (gegen - Akk)

$01(4$ 4:SE mit obligatorischem
Korrelat

\section{verspäten}

sich/Akkverspäten (mit - Dat)

\section{versprechen}

versprechen
PO Ich habe mich [ouf dieser Seite zweimal] verschrieben.

PO Der Professor hat sich dem Problem der historischen objektivität verschrieben.

P1 Ich versichere dich der Wahrheit meiner Worte.

P1 Der Mann versichert (der Witwe) seine Anteilnahme. Der Mann versicherte <es > ihr, keinen Fehler gemacht zu haben.

Der Mann versichert <es>ihr, daß er in die Stadt gehen werde.

Der Mann versicherte <es> ihr, er gehe in die Stadt.

$\mathrm{E}_{1}$ meist indefinit oder $\mathrm{SE}$.

P1 Mein Vater hat seinen Wagen (gegen Unfallschaden) versichert.

INF+: Mein Vater hat sich dagegen versichert, den Schaden allein ersetzen su müssen.

DASS: Mein Vater hat sich dagegen versichert, daß er das Risiko allein tragen muß.

Po Die Mutter verspätet sich (mit dem Einkoufen).

P1 Die Regiemung verspricht der Bevölkerung stabile Preise. 


\section{1:SE mit fakultativem} Korrelat

INF+:

DASS :

HPTS :

sich/Akk versprechen

0

sich/Dat versprechen von-Dat

4:SE mit obligatorischem Korrelat

\section{verstecken \\ verstecken (vor-Dat) \\ verstehen \\ verstehen \\ 1:SE mit fakultativem Korrelat}

01

INF+:

01

verstehen
Sie haben <es > der Tochter versprochen, ihr ein Auto zu kaufen.

Sie haben <es> der Tochter versprochen, daß sie ein Auto bekommt.

Die Eltern haben <es> der Tochter versprochen, sie werde ein Auto bekommen.

PO Der Lehrer hat sich versprochen.

PO Ich verspreche mir von dem neuen Auto mehr Sicherheit. INF+: Der Lehrer verspricht sich nichts davon, den Schüler zu schlagen.

- DASS : $\quad$ Ieh verspreche mir niceht viet davon, daß die Verhandlung vertagt worden ist.

P1 Die alte Frau versteckt ihr Geld (vor Dieben) (im Schrank).

: Der Dieb versteckt sich (vor der Polizei) (im Keller). i

P1 Hans versteht seine Arbeit [nicht].

Hans versteht <es>, ein Auto zu reparieren.

Mein Nachbar versteht $\langle e s\rangle$, wie man schnell zu Geld kommt.

P1 Ich verstehe deine Sorgen um die Zukunft. 
$1: S E$ mit fakultativem Korrelat

verstehen unter-Dat

verstehen von-Dat

014

BEMERKUNG :

verstehen

verstehen

018

sich/Akk verstehen auf-Akk

4:SE mit obligatorischem Korrelat

sich/Akk verstehen zu-Dat

4:SE mit obligatorischem Korrelat

sich/Akk verstehen (mit-Dat)

sich/Akk verstehen

08

\section{versuchen}

versuchen

01

versuchen

1:SE ohne Korrelat

versuchen
DASS :

FRAG :

Ich verstehe <es>, daß du zu viel zu tun hast.

Ich verstehe <es>, warum dir diese Krowatte nicht gefälzt.

P1 Unter Arbeit verstehe ich etwas anderes als sie.

PO Mein Freund versteht viel von Autos. $E_{1}$ meist indefinit.

P1 Deine Worte verstehe ich als eine Bitte um Hilfe.

P1 Ich habe deine Antwort falsch verstanden.

Po Hans versteht sich aufs Kochen.

INF+: Hans versteht sich darauf, Reis zu kochen.

INF+

PO Die beiden Parteien verstanden sich zu einem Kompromiß. Er verstand sich dazu, diese zusätzliche Aufgabe zu übernehmen.

PO Die beiden Kollegen verstehen sich (gut) (miteinander).

Po Der Mietpreis versteht sich ohne Nebenkosten.

P1 Der Teufel versucht den Menschen.

P1 Die Kinder versuchen den Kuchen.

FRAG: Die Kinder versuchen, ob der Kuchen gut ist. 


\section{1:SE ohne Korrelat}

versuchen es mit-Dat

sich/Akk versuchen an-Dat

sich/Akk versuchen in-Dat

4:SE mit obligatorischem Korrelat

sich/Akk versuchen

\section{verteilen}

verteizen (an - Akk)

verteizen auf - Akk

verteilen (auf - Akk)

verteizen (unter - Akk)

verteizen

verteizen
0115

INF+ :

FRAG :

04

04

04

07

$01(4$

014

$01(4$

01 ( 4

0116
Er versucht, ein Kotelett zu braten.

Ich versuche, ob der Automat funktioniert.

P2 Paul versucht es mit Arbeit.

PO Almut versucht sich an einer anderen Aufgabe.

PO Ingrid versucht sich im Schreiben.

INF+: Ingrid versucht sich darin, ein Auto zu fahren.

PO Maria versucht sich als Schauspielerin.

P1 Die Mutter verteizt den Kuchen (an die Kinder).

P1 Der Sportlehrer verteizt die Schüler auf awei Mannschaften.

P1 Er verteizt die Arbeit [gleichmäßig] (auf alle Mitarbeiter).

P1 Die Mutter verteizt den Kuchen (unter die Kinder). Er verteilt sein Geld (unter die Armen).

P1 Die Kinder haben die Plakate (in der ganzen Stadt) verteizt.

Die Gäste haben sich (im ganzen Haus) verteilt.

P1 Das Verkehrsamt hat die Wegweiser zum Schloß (über die ganze Stadt) verteilt.

Die Gäste haben sich (über das ganze Haus) verteizt. 


\section{verwechseln}

verwechseln (mit-Dat)

verzeihen

verzeihen

1:SE mit fakultativem Korrelat

\section{verzollen}

verzollen

\section{vorbereiten}

vorbereiten

vorbereiten auf-Akk

\section{$4: \mathrm{SE}$ mit obligatorischem Korrelat}

01

\section{vorlesen}

vorlesen
014

INF+ :

DASS :

$01(4$

01v3

DASS :

$0(1) 3$
P1 Fita verwechselte die beiden Brüder (miteinander).

P1 Ich verzeihe dir deinen Fehler. Ich verzeihe deinen Fehler. Ich verzeihe dir. Ich verzeithe <es > dir, daß du einen Fehler gemacht hast.

P1 Die Tante verzolzt den Kaffee und die Zigaretten.

P1 Die Mutter bereitet das Essen vor.

P1 Der Arzt bereitet den Kranken auf das Schlimmste vor. Der Student bereitet sich auf das Examen vor. Der Ingenieur bereitet sich darauf vor, an der Konferenz tei Inehmen zu müssen.

Der Arzt bereitet den Kranken darauf vor, daß sich das Schlimmste ereignen kann.

P1 Die Lehrerin liest (den Kindern) (die Aufgaben) vor. 
1:SE mit fakultativem Korrelat

DASS :

FRAG :

HPTS :

vorlesen (aus-Dat)

\section{vorstellen}

vorstezlen

vorstezlen

sich Dat vorstellen

1:SE mit fakultativem Korrelat

$01(3$

$01(5$

01

INF+ :
Anton liest <es> seinem Bruder vor, daß der FC Koln Bayem München 3 : 0 geschlagen hat.

Anton liest <es> seinem Bruder vor, welche FuBbalzmannschaft gewonnen hat.

Anton liest <es> seinem Bruder vor, der FC Köln habe Bayem Milnchen 3 : O geschlagen.

P2 Ich Zese (dir) (aus der Zeitung) vor.

DASS :

HPTS :
P1 Ich stelle (dem Chef) einen neuen Mitarbeiter vor.

P1 Peter stelzt seine Freundin (bei seinen Eltern) vor. Ich stelle mich (bei meinem neuen Chef) vor.

PO Ich stelle mir das Bizd vor.

Ich stelle <es> mir oft vor, meinen Vater wiederzusehen.

Ich kann <es > mir gut vorstellen, daß mein Vater kommen wird.

Ich stelle <es > mir vor, mein Vater kommt. 


\section{wachsen}

wachsen

Pertinenzelement im Dativ wachsen

BEMERKUNG :

\section{wachsen}

wachsen

BEMERKUNG :

\section{wagen}

wagen

$1:$ SE mit fakultativem Korrelat

sich/Akk wagen an-Akk

4:SE mit obligatorischem Korrelat

\section{wählen}

wählen

wählen

$0(1$

wählen in-Akk
INF+:

P1 Der Junge wagt einen Streit.

Mir wächst ein Bart.

Po Diese Pflanze wächst nur in tropischen Gebieten. STAMMFORMEN: wachsen, wuchs, gewachsen.

P1 Sie wachst ihr Auto. STAMMFORMEN: wachsen, wachste, gewachst. Der kleine Junge wagt <es> nicht, ins Wasser zu springen.

PO Der Student hat sich an ein sehr schwieriges Thema gewagt.

INF+: Ich habe mich nicht daran gewagt, dieses Thema zu behandeln.

P1 Der Geschäftsmann wählt nur die beste Ware.

P1 Die Gemeinde wählt (einen Bürgermeister).

P1 Die Arbeiter wählen ihren Kollegen in den Betriebsrat. 
wählen zu-Dat

014

wählen unter-Dat

04

wählen zwischen-Dat

04

\section{warten}

warten

warten mit-Dat

4:SE mit obligatorischem Korrelat

warten (ouf - Akk)

4:SE mit obligatorischem Korrelat

warten (ouf - Akk)

\section{waschen}

waschen

Pertinenzelement im Dativ waschen

\section{wechseln}

wechseln
INF+:

DASS :

FRAG :

$O(4) 5$

01
P1 Die Gemeinde wählt Herrn Schmitz zum Bürgermeister.

P2 Der Kunde wählt unter den verschiedenen Angeboten.

P2 Der Kunde wählt zwischen drei verschiedenen Tabaksorten.

P1 Der Monteur wartet die Maschine.

P2 Wir warten mit dem Essen.

INF+: Die Regierung wartet noch domit, diesen Vertrag zu unterzeichnen.

P2 Ich warte (auf Post). Die Gäste warten darauf, vorgestellt zu werden. Die Gäste warten darauf, daß sie vorgestell.t werden. Die Gäste warten darauf, ob sie vorgestellt werden.

po Er wartet (an der Normaluhr) (auf seine Freundin).

P1 Ich wasche mich.

Ich wasche mir das Gesicht.

P1 Karin wäscht (ihre Strümpfe).

Po Die Zeiten wechseln.

Ihre Stimmung wechselt [häufig]. 
wechsein

01

wechseln (in - Akk)

$01(4$

wechseln (mit-Dat)

$01(4$

BEMERKUNG :

\section{wechseln}

wechseln

BEMERKUNG :

\section{wecken}

wecken

wecken (aus - Dat)

$01(4$

wecken

\section{weinen}

weinen

weinen ( ïber-Akk)

$0<4$

4:SE mit obligatorischem Korrelat

weinen ( um- Akk)
P1 Ich werde meinen Beruf wechseln.

P1 Der Kaufmann wechselt [mir] das Markstück (in Zehnpfennigstücke).

P1 Wir wechseln [regelmäßig] Briefe (mit unseren Freunden).

STAMMFORMEN: wechseln, wechselte, hat gewechselt.

P2 Das Reh wechselt in den Wald.

STAMMFORMEN: wechseln, wechselte, ist gewechselt.

P1 Die Mutter weckt ihre Tochter.

P1 Das Telefon hat mich (aus dem Schlaf) geweckt.

P1 Dieser Film weckte Erinnerungen (in mir).

P1 Sie weint(heiße Trönen).

P2 Sie weint (ïber seinen Tod).

INF+: $\quad$ Sie weint darïber, verlassen worden zu sein.

DASS: $\quad$ Sie weint daruber, daß ihr Mann sie verlassen hat.

o(4 P2 Sie weint (um den Freund). 
wenden

wenden

wenden

\section{BEMERKUNG :}

\section{wenden}

wenden

sich/Akk wenden an - Akk

sich/Akk wenden gegen-Akk

4:SE mit obligatorischem Korrelat

sich/Akk wenden zu-Dat BEMERKUNG :

\section{werden}

werden aus - Dat

werden zu-Dat

O4

werden

07

werden
01

o(1

01 ( 6

04

04

INF+:

DASS :

P1 Die Mutter wendet den abgetragenen Mantel.

P1 Hans wendet (sein Auto).

STAMMFORMEN: wenden, wendete, gewendet.

PO Der Gast wendet den Kopf (zur Tür).

Po Die Arbeiter wenden sich an den Betriebsrat.

PO Die Schüler wenden sich gegen ihren Lehrer. Die Mitarbeiter wenden sich gegen die neue Vor schmift.

Der Arbeiter wendet sich dagegen, an der neuen Maschine arbeiten zu müssen.

Der Gast wendet sich dagegen, daß er ein Zimmer ohne Bad bekommen solz.

PO Die Hausfrau wendet sich zu ihren Gästen. STAMMFORMEN: wenden, wandte, gewandt.

PO Aus unseren Urlaubsplänen ist nichts geworden.

PO Er wurde [durch den Tod seiner Eltern] zum Dieb.

PO Angelika wird Lehrerin.

PO Das Wetter wird schlechter. 


\begin{tabular}{|c|c|c|c|}
\hline VERB & SBP & & BEISPIELSATZ \\
\hline werden & 38 & PO & Mir wird schlecht́. \\
\hline werden (es) & 7 & PO & Es wird Tag. \\
\hline werden (es) & 8 & PO & Es ist spät geworden. \\
\hline \multicolumn{4}{|l|}{ werfen } \\
\hline werfen & 01 & P1 & $\begin{array}{l}\text { Der Baum wirft einen langen Schatten. } \\
\text { Der Finne hat einen neuen Weltrekord geworfen. }\end{array}$ \\
\hline werfen & $0(1$ & P1 & Unsere Katze hat (drei Junge) geworfen. \\
\hline werfen & 016 & P1 & $\begin{array}{l}\text { Der Mann wirft seine Gäste aus dem Haus. } \\
\text { Du hast meinen Bleistift auf den Boden geworfen. } \\
\text { Das Kind wirft sich auf den Boden. }\end{array}$ \\
\hline Pertinenzelement im Dativ & & & Er wirft mir einen Stein an den Kopf. \\
\hline werfen mit-Dat & $04(6$ & PO & Die Kinder werfen mit Steinen (auf das Auto). \\
\hline sich/Akk werfen auf-Akk & 04 & $\mathrm{PO}$ & Der junge Mann warf sich auf seine Arbeit. \\
\hline *einen Blick werfen auf - Akk & I & PO & $\begin{array}{l}\text { Das Kind warf nur einen kurzen Blick auf seine } \\
\text { Geschenke. }\end{array}$ \\
\hline *einen Blick werfen in - Akk & I & $\mathrm{PO}$ & Der Anwalt warf einen Blick in die Akten. \\
\hline *um sich werfen mit-Dat & I & $\mathrm{PO}$ & Er wirft mit fremden Wörtern um sich. \\
\hline \multicolumn{4}{|l|}{ wiederholen } \\
\hline wiederholen & 01 & P1 & Der Schüler wiederholt die Klasse. \\
\hline wiederholen & 01 & P1 & Der Schüler wiederholt den Satz. \\
\hline
\end{tabular}




\section{1:SE mit fakultativem Korrelat}

sich/Akk wiederholen

wiegen

wiegen

wiegen

wiegen

BEMERKUNG :

\section{wiegen}

wiegen

*in Sicherheit wiegen

BEMERKUNNG:

\section{winken}

winken

winken

winken
0

DASS:

HPTS :

01

01

08

016

013

016
Der Schüler wiederholt <es>, daß er seine Aufgaben gemacht hat.

Der Schüler wiederholt <es >, er habe seine Aufgaben gemacht.

Po Der Redner hat sich [oft] wiederholt.

Po Das Fleisch wiegt drei Pfund.

P1 Der Bauer wiegt die Kartoffeln. Das Kind wiegt sich [jeden Morgen].

Po Dieser Fehler wiegt schwer. STAMMFORMEN: wiegen, wog, gewogen.

P1 Der Vater wiegt das Kind [in seinen Armen].

P1 Der Betrüger wiegt sein Opfer in Sicherheit. STAMMFORMEN: wiegen, wiegte, gewiegt.

P1 Der Polizist winkt den Autofahrer an die Seite.

P2 Der Autofahrer winkt (dem Fußgänger).

P2 Die Kinder winken (ous dem Fenster). 


\section{wissen}

wissen

1:SE mit fakultativem Korrelat

01

DASS :

FRAG

HPTS :

\section{BEMERKUNG :}

wissen (von - Dat)

$01(4$

4:SE mit obligatorischem Korrelat

wissen um-Akk

4:SE mit fakultativem Korrelat

〈sich/Dat> wissen

\section{wohnen}

wohnen

DASS :

04
P1 Meine Schwester weiß sehr viez.

Meine Schwester weiß <es> genau, daß Vater später kommt.

Meine Schwester weiß <es > nicht, wann Vater zurückkommt.

Meine Schwester weiß<es> genau, Vater komnt heute später.

$E_{1}$ meist indefinit oder $\mathrm{SE}$.

PO Ich weiß nichts (von diesem Vorfall).

Ich weiß nichts davon, daß ex morgen kommen will.

Ich weiß nichts davon, ob er morgen kommen kann.

PO Der alte Mann wußte um das Geheimnis.

DASS: . Der alte Mann wußte <darum>, daß auf der Insel ein Schatz vergraben war.

FRAG: Der alte Mann wußte <damum, wo der Schatz vergraben war.

PO Ich wei $<$ mir > keinen Rat [mehr].

PO In diesem Haus wohnen drei Familien zur Miete.

[In Deutschland] wohnen viele Menschen in Großstädten.

Meine Eltern wohnen zur Miete. 


\section{wundern}

wundern

O:SE Zum Korrelat vgl. Einleitung 6.3 .5 .

sich/Akk wundern (uber-Akk)

014

4:SE mit fakultativem Korrelat

01

DASS :

DASS :

FRAG:

\section{wïschen}

winschen

1:SE mit fakultativem Korrelàt

01

INF+:

DASS :

winschen

\section{1: SE mit fakultativem} Korrelat
PO Dieser Vorschlag wundert mich.

Es wundert mich, daß du soviel tminkst.

PO Anne wundert sich über ihren Bruder.

Anne wundert sich <dariber>, daß ihr Bruder sich so veröndert hat.

Alze wundern sich <dariber>, wie Barbara sich: verändert hat.

P1. Vater wïnscht eine Erkzärung.

Vater wïnscht <es > schon Zange, eine Erklärung für das Verhalten seines Sohnes zu bekommen.

Sein Vater hat <es > schon immer gewilnscht, daß seine Tochter Lehrerin wird.

P1 Die Eltern wünschen dem jungen Paar viel Glück. Seine Frau wïnscht sich ein eigenes Auto.

DASS: $\quad$ Die Eltern wünschen <es > ihren Kindern von Herzen, daß sie glücklich werden. 


\section{zahlen}

zahlen

zahlen (an - Akk)

zahlen

zahlen

$\mathrm{O}(18$

\section{zählen}

zählen

zählen

zählen unter - Akk

014

zähZen zu-Dat

$1: \mathrm{SE}$ Zum Korrelat vgl. Einleitung 6.3 .5 .

zählen

016

zählen
P1 Herr Schneider zahlt (dem Vermieter) eine hohe Miete.

P1 Herr Schneider zahlt (an seinen Vermieter) eine hohe Miete.

P1 Er zahlt hundert Mark (in die gemeinsame Kasse).

P1 Er zahlt (die Waschmaschine) bar.

Er zahlt (die Waschmaschine) in Raten.

Po Die Stunde zählt sechzig Minuten.

P1 Die Kinder zählen die Bücher.

P1 Man zählt [häufig] die Türkei unter die europäischen Staaten.

P1 Goethe zählte Schiller zu seinen Freunden. Goethe zählte sich zu Herders Freunden.

INF+: Sie zählt es zu den wichtigsten Ereignissen in ihrem Leben, diesem Mann begegnet zu sein.

DASS: $\quad$ Ich zähle es zu meinen unangenehmsten Erfahrungen, daß mir einmal mein ganzes Geld gestohlen worden ist.

P1 Der Makler zählte das Geld ouf den Tisch.

P1 Der Rechtsanwalt zählt diesen Fall als Erfolg. 
1:SE Zum Korrelat vgl. Einleitung 6.3.5.

zählen

zählen auf - Akk

$4:$ SE mit obligatorischem Korrelat

zählen (bis) .

zählen zu-Dat

zählen

O:SE Zum Korrelat vgl. Einleitung 6.3.5.

zählen

018

\section{zeichnen}

zeichnen

zeichnen

zeichnen

04

$O(4$

04

07

018
INF+:

Er zählt es als seinen schönsten. Erfolg, diesem Menschen das Leben gerettet zu haben.

DASS :

$01(8$

INF+:

DASS :

\section{INF+ :}

DASS :

$0(1) 6$

Er zählt es als seinen schönsten Erfolg, daß er diesen Menschen gerettet hat.

P1 Wir zählen Eier (im Dutzend).

P2 Ich zähle auf meinen Freund.

Der Arzt zählt darouf, Hans zu sehen.

Er zählt darauf, daß bei der Abstimmung alle anwesend sind.

P2 Das Kind kann (bis hundert) zählen.

PO Goethe zählt zu den bedeutendsten Dichtern.

PO. Der erste Mai zählt als Feiertag.

Es zählt als schweres Vergehen, Verletzten nicht zu helfen.

Es zählt als Verstoß gegen die Straßenverkehrsordnung, daß du betrunken Auto gefahren bist.

Po Jede Stimme zählt (doppelt).

P1 Die Frau zeichnet die Wäsche.

Der Spekulant zeichnet Aktien.

P1 Die Kinder zeichnen (Blumen) (an die Wand).

P1 Er zeichnet den Baum abstrakt. 


\section{zeigen \\ zeigen \\ zeigen \\ 1:SE mit fakultativem Korrelat \\ zeigen \\ sich/Akk zeigen \\ O:SE Zum Korrelat vgl. Einleitung 6.3.5.}

sich/Akk zeigen

08

\section{ziehen}

ziehen (es)

ziehen

ziehen

0

01

Pertinenzelement im Dativ ziehen

Pertinenzelement im Dativ ziehen
PO Die Uhr zeigt halb zehn.

P1 Er zeigt (uns) seine Arbeit.

DASS: $\quad$ Er zeigt <es> uns, daß der arbeitet.

FRAG: $\quad$ Er zeigt <es> uns, wo er wohnt.

PO Die Kompaßnadel zeigt nach Norden.

PO [Trotz unserer vielen Arbeit] zeigt sich [noch] kein Erfolg.

DASS: Es hat sich gezeigt, daß sie recht hatten.

FRAG: $\quad$ Es wird sich noch zeigen, wie das Problem gelöst werden kann.

PO Er zeigt sich großzügig.

PO Es zieht.

PO Der Tee zieht.

P1 Du hast ein schlechtes Los gezogen. Der Zahnarzt hat mir den Zahn gezogen.

P1 Der Mann zog das Kind aus dem Wasser. Er zog sich die Decke über den Kopf.

P1 Das Pferd zieht den Wagen (durch den Wald). 
*in Erwägung ziehen

*ins Vertrauen ziehen

*in Zweifel ziehen

T

$\mathrm{T}$

*zu Rate ziehen

$\mathrm{T}$

*zur Rechenschaft ziehen

*zur Verantwortung ziehen

$\mathrm{T}$

$\mathrm{T}$

BEMERKUNG :

\section{ziehen}

ziehen

ziehen

015

06

BEMERKUNG :

\section{zuhören}

zuhoren

\section{zumachen}

zumachen

Pertinenzelement im Dativ zumachen
P1 Wir ziehen den Kauf eines Wohrwagens in Erwägung.

P1 Der Chef zog seine Mitarbeiter ins Vertrauen.

P1 Der Richter zog die Aussage des Zeugen in Zweifel.

P1 Der Geschädigte zog einen Anwalt zu Rate.

P1 Hans zog den Betrüger zur Rechenschaft.

P1 Der Vater zog seinen Sohn zur Verantwortung. STAMMFORMEN: ziehen, zog, hat gezogen.

PO Wolken ziehen (am Himmel).

P2 Die Vögel ziehen nach Süden. STAMMFORMEN: ziehen, zog, ist gezogen.

P2 Die Kinder hören (der Großmutter) zu.

P1 Barbara macht das Fenster zu. Er macht ihr das Kleid zu.

o15 P2 Die Geschäfte machen (um achtzehn Uhr) zu. 


\section{zweifeln}

zweifeln (an - Dat)

$0(4$

4:SE mit fakultativem Korrelat

\section{4:SE mit obligatorischem} Korrelat
P2 Karin zweifelt (an der Liebe ihres Freundes).

Die junge Frau zweifelt <daran〉, daß ihr Mann sie liebt.

Sie zweifelt <daran>, ob sie von ihrem Mann geliebt wird.

INF+:

Sie zweifelt daran, von ihrem Mann geliebt zu werden. 

